


\begin{abstract}
Aus der Abteilung Klinische Neurowissenschaften (Prof. Dr. med. Dr. med. vet. H. Ehrenreich) des Max-Planck-Instituts für Experimentelle Medizin in Göttingen
\end{abstract}

\title{
Assoziation von Höhe der antipsychotischen Medikation über die Zeit mit Kognition schizophrener Patienten unter Berücksichtigung des Geschlechts
}

\author{
INAUGURAL-DISSERTATION \\ zur Erlangung des Doktorgrades \\ der Medizinischen Fakultät \\ der Georg-August-Universität zu Göttingen
}

vorgelegt von

Christoph Johannes Szuszies

aus Bielefeld

Göttingen 2011 
Dekan:

I. Berichterstatterin:

II. Berichterstatter/in:

III. Berichterstatter/in:
Prof. Dr. med. C. Frömmel

Prof. Dr. med. Dr. med. vet. H. Ehrenreich

Prof. Dr. sc. hum. Friede

Tag der mündlichen Prüfung: $\quad 06.06 .2012$ 


\section{INHALTSVERZEICHNIS}

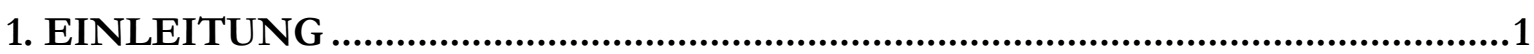

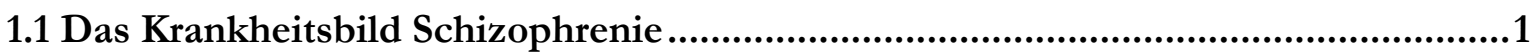

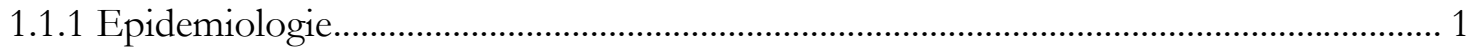

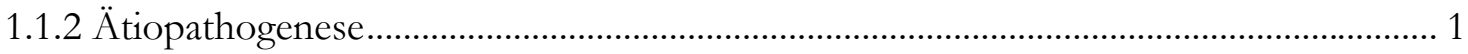

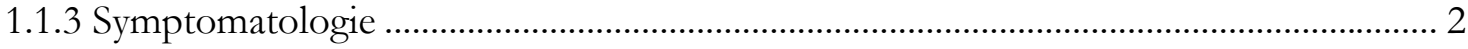

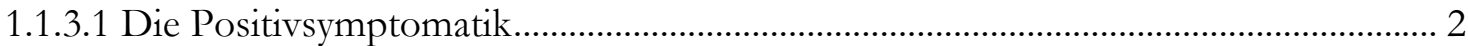

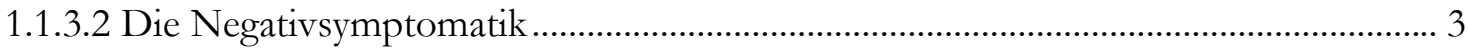

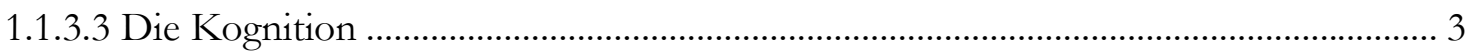

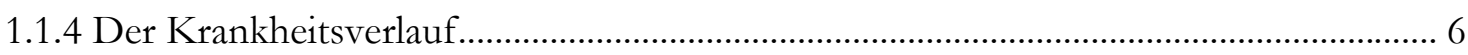

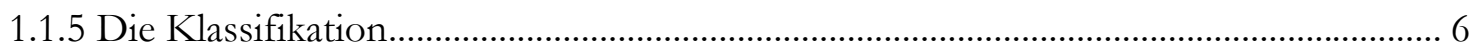

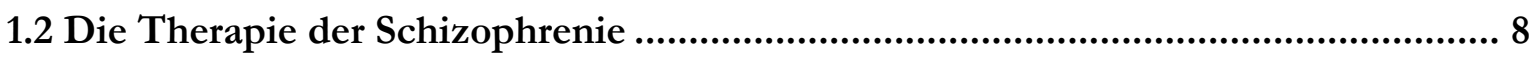

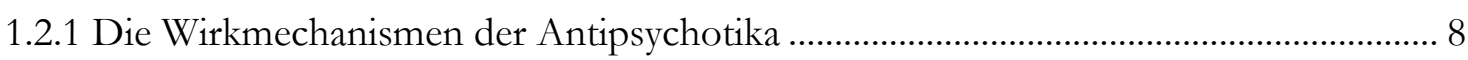

1.2.1.1 Assoziation von antipsychotischer Behandlung mit Kognition................................ 10

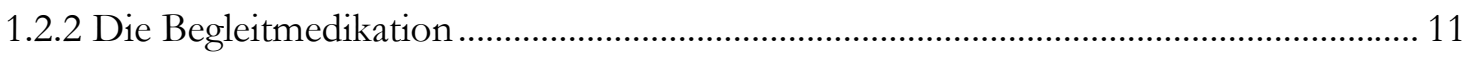

1.2.2.1 Anticholinerge Medikation ......................................................................................... 11

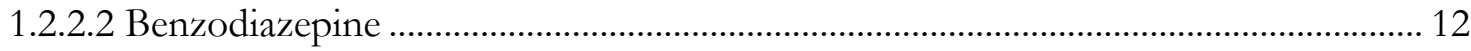

1.2.2.3 Nichtmedikamentöse Behandlungsansätze ........................................................................ 12

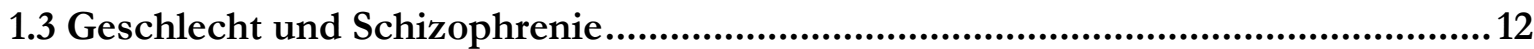

1.3.1 Geschlechtsspezifischer Verlauf der Schizophrenie .......................................................... 13

1.3.2 Geschlechtsspezifische Unterschiede in der Kognition ................................................. 14

1.3.3 Geschlechtsspezifische Unterschiede in der antipsychotischen Therapie.................... 15

1.3.4 Geschlechtsunterschiede in den Nebenwirkungen der Antipsychotika ........................ 16

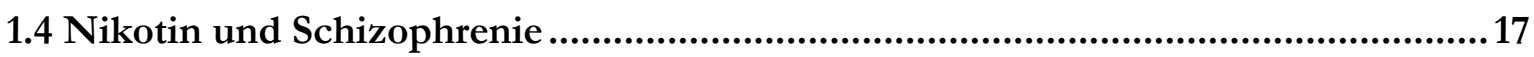

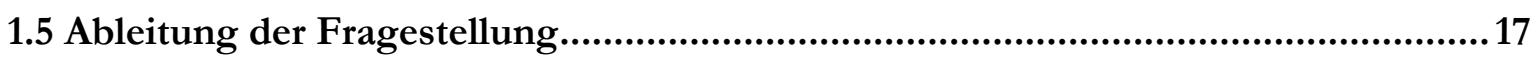

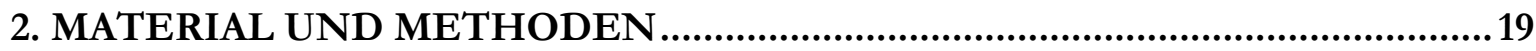

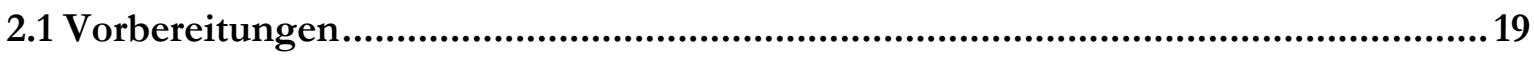

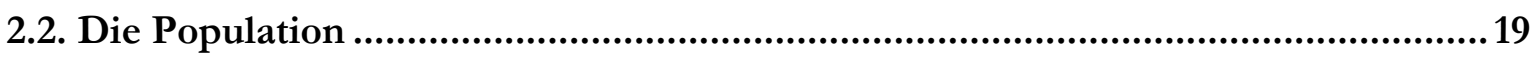

2.3. Verwendete Untersuchungsinstrumente .........................................................20

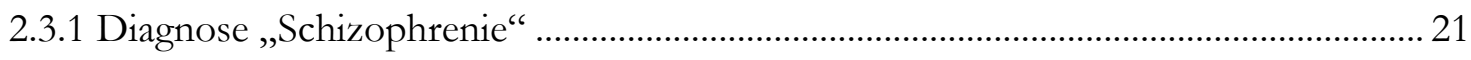

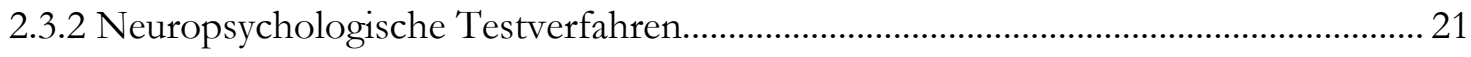

2.3.3. Skalen zur Beurteilung klinischer Variablen..................................................................... 24

2.3.3.1 Neurologische Beurteilungsskala ................................................................................... 25

2.3.3.2 Skala zur Beurteilung der Krankheitsschwere ............................................................. 25 
2.3.3.3 Skala zur Beurteilung des Funktionsniveaus ................................................................. 26

2.3.3.4 Skala zur Beurteilung der Psychopathologie .................................................................. 26

2.3.3.5 Skalen zur Einschätzung der Medikamentennebenwirkungen .................................... 26

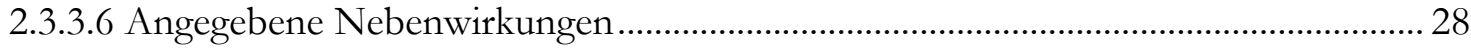

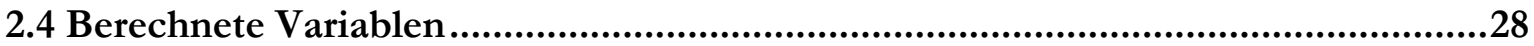

2.4.1 Antipsychotische Medikation und das Chlorpromazinäquivalent ..................................2 28

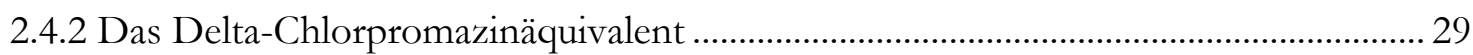

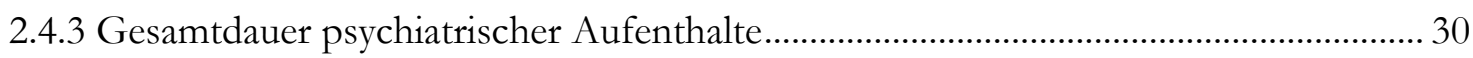

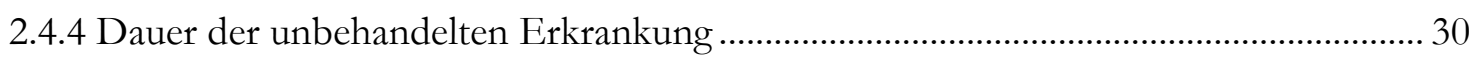

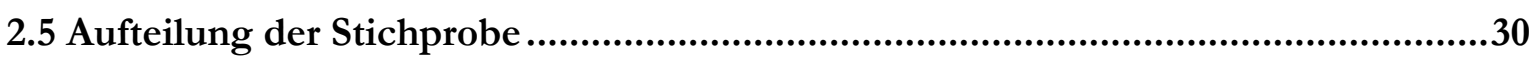

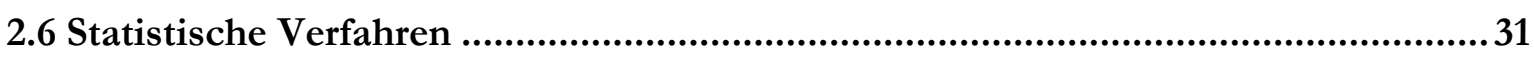

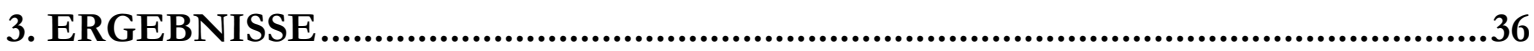

3.1 Verteilung der antipsychotischen Dosis zum GRAS-Untersuchungszeitpunkt .......36

3.2 Verteilung der antipsychotischen Dosis zum Zeitpunkt der Erstmedikation ..........38

3.3 Vergleich der antipsychotischen Dosissteigerung zwischen Männern und Frauen40

3.3.1 Geschlechtsunterschiede in der Ausprägung von Variablen, die mit der Höhe der antipsychotischen Tagesdosis assoziiert sind ...................................................................... 41

3.4 Assoziation der antipsychotischen Dosissteigerung mit der Kognition ...................43

3.4.1 Kognitive Gruppenunterschiede in der Stichprobe der Männer ................................... 43

3.4.2 $\Delta \mathrm{CPÄ/J-Gruppenunterschiede} \mathrm{kognitionsmodulierender} \mathrm{Variablen} \mathrm{in} \mathrm{der}$

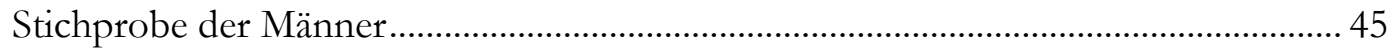

3.4.3 Kognitive Gruppenunterschiede in der Stichprobe der Frauen ................................... 47

3.4.4 $\Delta$ CPÄ/J-Gruppenunterschiede kognitionsmodulierender Variablen in der

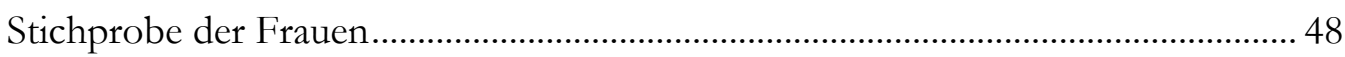

3.5 Assoziation antipsychotischer Dosissteigerung pro Jahr mit Kognition unter

Berücksichtigung des Alters ....................................................................50

3.5.1 Unterschiede in der Stichprobe der Männer.................................................................... 51

3.5.2 Unterschiede in der Stichprobe der Frauen .................................................................. 52

3.6 Betrachtung klinischer und medikamentöser Gruppenunterschiede in den jeweiligen Subgruppen .................................................................................52

3.6.1 Gruppenunterschiede in den Medikamentennebenwirkungen ...................................... 52

3.6.2 Gruppenunterschiede in der Komedikation ..................................................................... 54

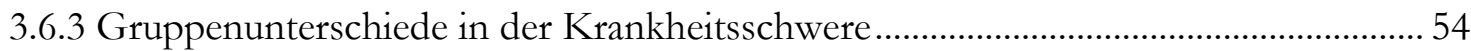

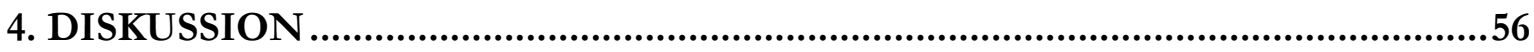


4.1 Geschlechtsunterschiede der antipsychotischen Dosis..........................................57

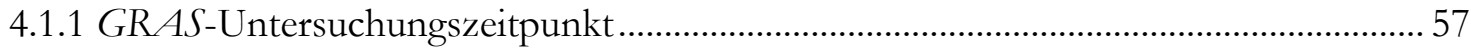

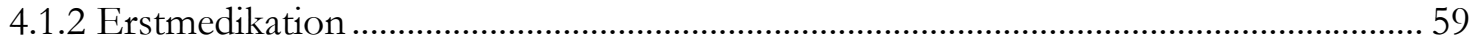

4.2 Geschlechtsunterschiede der antipsychotischen Dosissteigerung über die Medikationsdauer .............................................................................................60

4.3 Assoziation antipsychotischer Dosissteigerung mit der Kognition ........................65

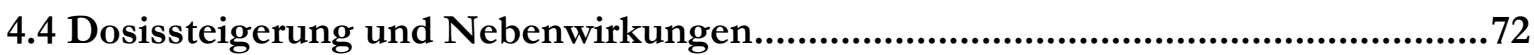

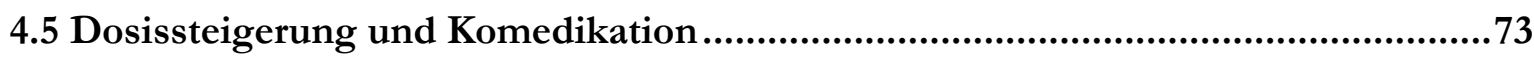

4.6 Dosissteigerung und Krankheitsschwere ...............................................................74

4.7 Stärken der vorliegenden Arbeit und klinische Relevanz der Befunde.....................77

4.8 Kritische Diskussion der verwendeten Methoden und Forschungsausblick............ 81

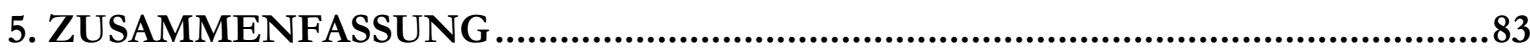

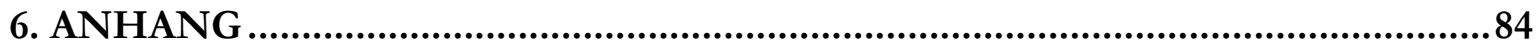

Anhang A: Übersicht über die teilnehmenden Zentren der GRAS-Studie ............................. 84

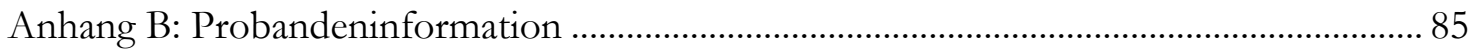

Anhang C: Übersicht über die in der GRAS-Studie verwendeten psychometrischen, neuropsychologischen und neurologischen Messinstrumente.................................... 86

Anhang D: Kategorieneinteilung der von Patienten genannten Nebenwirkungssymptome87

Anhang E: Tabelle der in Depotform applizierten AP mit verwendeten Umrechnungs-

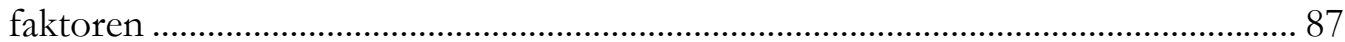

Anhang F: Tabelle oral applizierter AP mit verwendeten Umrechnungsfaktoren ............... 88

Anhang G: Häufigkeitsverteilung des Alters zum Zeitpunkt der GRAS-Untersuchung.... 89

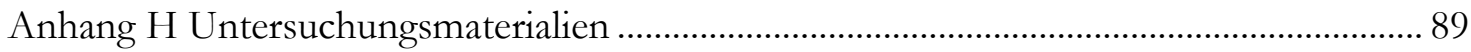

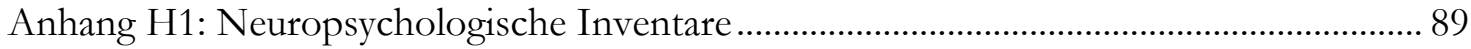

Anhang H2: Skala zur Enschätzung der Psychopathologie .................................................. 93

Anhang H3: Skalen zur Einschätzung der Erkrankungsschwere .......................................... 93

Anhang H4: Inventar zur Untersuchung der neurologischen Erkrankungsschwere ........... 94

Anhang H5: Inventare zur Untersuchung der Nebenwirkungen antipsychotischer Therapie. 


\section{VERZEICHNIS DER VERWENDETEN ABKÜRZUNGEN}

a

AIMS

ANCOVA

AP

BAS

BZT

bzw.

CATIE

CET

CGI

$\mathrm{CNI}$

CPÄ

CPK

CYP P 450

d

$\triangle C P \ddot{A}$

d.h.

DISC1

$\mathrm{D}_{2}-\mathrm{R}$

DSM-IV-TR

DUP

EPMS

etc.

f.

FGA

GABA

GAF

GRAS

HVA

ICD-10

IQ

Kap.

KST

K-S-Z

LPS3

M

MATRICS

$\operatorname{Max}$

MD annus, Jahr

Abnormal Involuntary Movement Scale

Analysis of Covariance (Kovarianzanalyse)

Antipsychotikum, Antipsychotika

Barnes-Akathisia Scale

Buchstaben-Zahlen-Test

beziehungsweise

Clinical Antipsychotic Trials in Intervention Effectiveness

Cognitive Enhancement Therapy

Clinical Global Impression Scale

Cambridge Neurological Inventory

Chlorpromazinäquivalent

Chlorpromazinkoeffizient

Cytochrom P 450

dies, Tag

Delta-Chlorpromazinäquivalent (Differenz der Chlorpromazinäquivalente der Tagesdosen zwischen Erstmedikation und Medikation zum GRASUntersuchungszeitpunkt)

das heißt

disrupted in schizophrenia 1

Dopamin-D2-Rezeptor

Diagnostic and Statistical Manual of Mental Disorders $4^{\text {th }}$ Edition "Text Revision"

Dauer der unbehandelten Psychose vor der Erstmedikation

extrapyramidal-motorische Störungen

et cetera

folgende

first generation antipsychotics, Typikum

$\gamma$-Aminobuttersäure

Global Assessment of Functioning Scale

Göttinger Research Association for Schizophrenia

Homovanillinsäure

International Classification of Diseases $10^{\text {th }}$ Revision

Intelligenzquotient

Kapitel

Kolmogorov-Smirnov-Test

Kolmogorov-Smirnov-Z

Leistungsprüfsystem Subtest 3

Mittelwert

Measurement and Treatment Research to Improve Cognition in Schizophrenia

Maximum

mittlere absolute Abweichung vom Median 


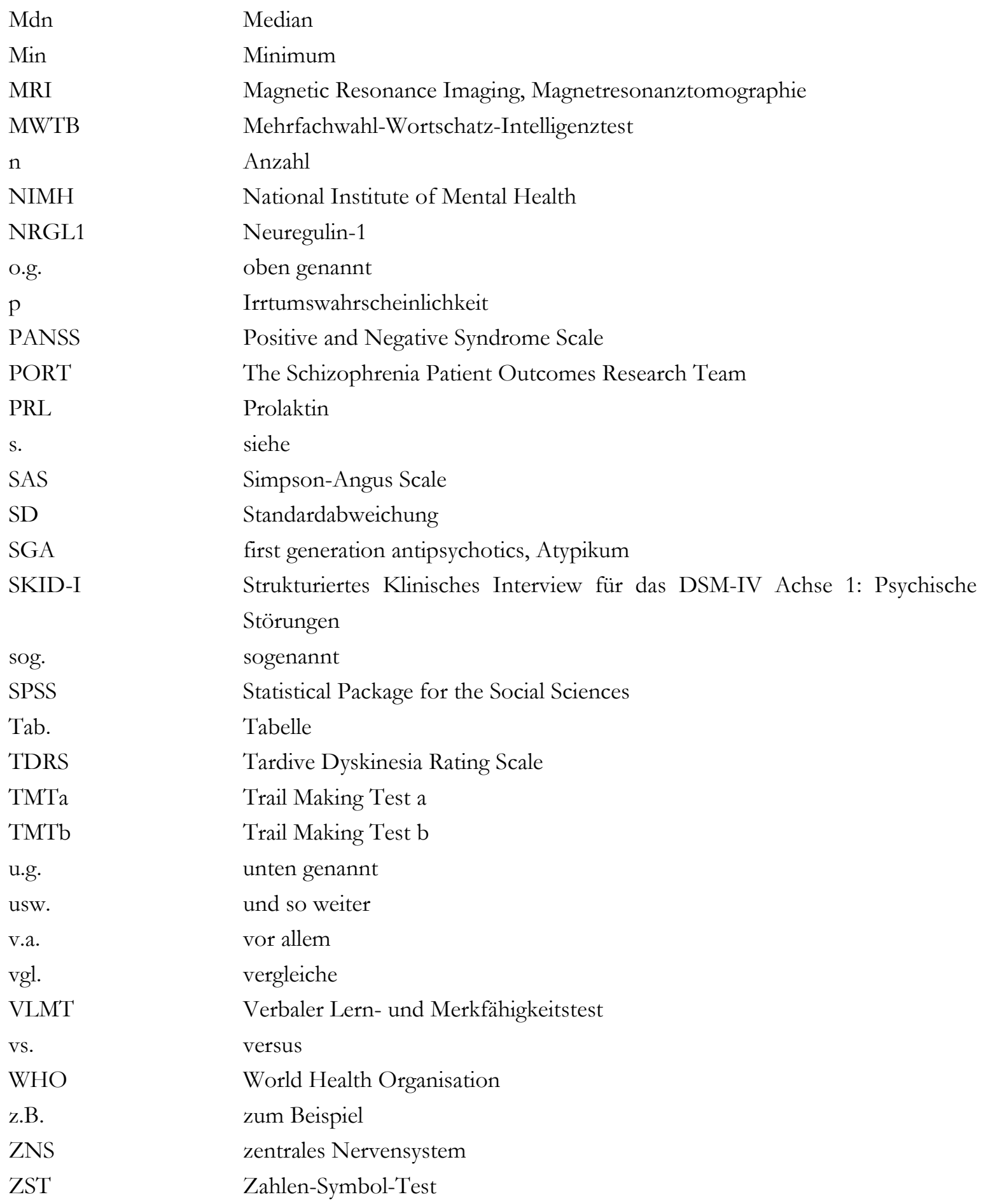

Zeichen für statistische Fehlerwahrsacheinlichkeiten:

$* \mathrm{p}<0,05 \quad * * \mathrm{p}<0,01 \quad * * * \mathrm{p}<0,001 *$ Signifikanz nach Bonferroni-Korrektur

Anmerkung: Zur besseren Lesbarkeit des Textes wird in der vorliegenden Arbeit als Personenbezeichnung jeweils die männliche Sprachform gewählt. Sie ist aber stets geschlechtsneutral zu interpretieren und schließt Frauen wie Männer gleichermaßen ein. 


\section{ABBILDUNGSVERZEICHNIS}

Abbildung 1: Verteilung der antipsychotischen Tagesdosen in Chlorpromazinäquivalenten (CPÄ) zum Zeitpunkt der GRAS-Untersuchung in der Gesamtstichprobe

Abbildung 2: Verteilung der antipsychotischen Tagesdosen in Chlorpromazinäquivalenten (CPÄ) zum Zeitpunkt der GRAS-Untersuchung in der Stichprobe der Männer.

Abbildung 3: Verteilung der antipsychotischen Tagesdosen in Chlorpromazinäquivalenten (CPÄ) zum Zeitpunkt der GRAS-Untersuchung in der Stichprobe der Frauen

Abbildung 4: Gegenüberstellung der Medianwerte der antipsychotischen Tagesdosen in Chlorpromazinäquivalenten (CPÄ) von der Gesamt-, Männer- und Frauenstichprobe zum GRAS-Untersuchungszeitpunkt

Abbildung 5: Verteilung der antipsychotischen Tagesdosen in Chlorpromazinäquivalenten (CPÄ) zum Zeitpunkt der Erstmedikation in der Gesamtstichprobe...

Abbildung 6: Verteilung der antipsychotischen Tagesdosen in Chlorpromazinäquivalenten (CPÄ) zum Zeitpunkt der Erstmedikation in der Stichprobe der Männer

Abbildung 7: Verteilung der antipsychotischen Tagesdosen in Chlorpromazinäquivalenten (CPÄ) zum Zeitpunkt der Erstmedikation in der Stichprobe der Frauen.

Abbildung 8: Gegenüberstellung der Medianwerte der antipsychotischen Tagesdosen in Chlorpromazinäquivalenten (CPÄ) von der Gesamt-, Männer- und Frauenstichprobe zum Zeitpunkt der Erstmedikation.

Abbildung 9: Gegenüberstellung der Medianwerte der antipsychotischen Dosissteigerung pro Jahr in Chlorpromazinäquivalenten $(\triangle \mathrm{CPÄ} / \mathrm{J})$ von der Gesamt-, Männer- und Frauenstichprobe.

Abbildung 10: Neuropsychologische Testunterschiede zwischen den $\Delta C P \ddot{A} / J$-Gruppen in der Stichprobe der Männer

Abbildung 11: Neuropsychologische Testunterschiede zwischen den $\Delta$ CPÄ/J-Gruppen in der Stichprobe der Frauen

Abbildung 12: Schematische Darstellung der Assoziation von Dosissteigerung pro Jahr Medikationsdauer, kognitiver Beeinträchtigung und Krankheitsschwere 


\section{TABELLENVERZEICHNIS}

Tabelle 1: Kriterien zur Diagnose der Schizophrenie nach DSM-IV-TR ................................................. 7

Tabelle 2: Charakterisierung der untersuchten Stichprobe anhand wesentlicher Variablen

Tabelle 3: Deskriptive und inferenzstatistische Werte der mittleren CPÄ-Tagesdosis zum GRASUntersuchungszeitpunkt .................................................................................................... 37

Tabelle 4: Deskriptive und inferenzstatistische Werte zur mittleren CPÄ-Dosis zum GRASUntersuchungszeitpunkt

Tabelle 5: Gegenüberstellung deskriptiver und inferenzstatistischer Werte der durchschnittlichen antipsychotischen Dosissteigerung pro Jahr $(\triangle \mathrm{CP} \ddot{A} / \mathrm{J})$ zwischen der Männer-, Frauenund Gesamtstichprobe

Tabelle 6: Charakterisierung der Stichproben hinsichtlich modulatorischer Größen der antipsychotischen Medikation

Tabelle 7: Unterschiede kognitiver Leistung zwischen den $\triangle \mathrm{CPÄ/J-Gruppen} \mathrm{in} \mathrm{der} \mathrm{Stichprobe} \mathrm{der}$ Männer.

Tabelle 8: Deskriptive und inferenzstatistische Unterschiede kognitionsmodulierender Variablen zwischen den $\triangle \mathrm{CP} \ddot{A} / \mathrm{J}$-Gruppen in der Stichprobe der Männer ..

Tabelle 9: Anzahl der applizierten AP zum GRAS-Untersuchungszeitpunkt und zum Zeitpunkt der Erstmedikation in der Stichprobe der Männer.

Tabelle 10: Unterschiede kognitiver Leistung zwischen den $\triangle \mathrm{CP} \ddot{\mathrm{A}} / \mathrm{J}-$ Gruppen in der Stichprobe der Frauen

Tabelle 11: Anzahl der applizierten AP zum GRAS-Untersuchungszeitpunkt und zum Zeitpunkt der Erstmedikation in der Stichprobe der Frauen.

Tabelle 12: Deskriptive und inferenzstatistische Unterschiede kognitionsmodulierender Variablen zwischen den $\Delta \mathrm{CP} \ddot{\mathrm{A}} / \mathrm{J}$-Gruppen in der Stichprobe der Frauen.

Tabelle 13: Kennwerte der ANOVA zur Prüfung der Assoziation des Alters mit den $\Delta \mathrm{CPÄ} / \mathrm{J}$ Gruppenunterschieden in neuropsychologischen Tests in der Stichprobe der Männer . 51

Tabelle 14: Kennwerte der ANOVA zur Prüfung der Assoziation des Alters mit den $\Delta \mathrm{CPÄ} / \mathrm{J}$ Gruppenunterschieden in neuropsychologischen Tests in der Stichprobe der Frauen... 52

Tabelle 15: Überprüfung der Gruppenunterschiede hinsichtlich neurologischer Nebenwirkungen in der Stichprobe der Männer und Frauen

Tabelle 16: Überprüfung der Gruppenunterschiede hinsichtlich von Probanden angegebener Nebenwirkungen in der Stichprobe der Männer und Frauen

Tabelle 17: Überprüfung der Gruppenunterschiede hinsichtlich der Komedikation in der Stichprobe der Männer und Frauen

Tabelle 18: Gruppenunterschiede zwischen den $\triangle \mathrm{CP} \ddot{\mathrm{A}} / \mathrm{J}$-Gruppen hinsichtlich der 


\section{EINLEITUNG}

\subsection{Das Krankheitsbild Schizophrenie}

Schizophrenien gehören zu den häufigsten psychiatrisch diagnostizierten Erkrankungen und beeinträchtigen die Betroffenen in Denken, Wahrnehmung und Affekt. Der aus dem altgriechischen stammende Begriff Schizophrenie ( $\sigma \chi i \zeta \varepsilon \iota \nu$ s'chizein „abspalten“ $\varphi \varrho \dot{\eta} \nu$ phrēn „Seele) geht auf Eugen Bleuler zurück, der damit die Aufspaltung der mentalen Funktionen zum Ausdruck bringen wollte; er erkannte die gestörte Einheit der Persönlichkeit, bedingt durch eine Zersplitterung des subjektiven Gefühls der Persönlichkeit, sowie eine Aufspaltung der psychischen Tätigkeiten des Fühlens, Denkens und Wollens (Bleuler 1911). Um die Heterogenität des Krankheitsbildes zu verdeutlichen, sprach Bleuler nicht von „der Schizophrenie“ sondern von der „Gruppe der Schizophrenien“. Heute spricht man von Erkrankungen aus dem schizophrenen Formenkreis, deren Gruppe eine Vielzahl komplexer Syndrome beinhaltet. Diese variieren in Bezug auf Ätiologie, Symptomatik, Verlauf und Therapierbarkeit (Andreasen 1995; Bayer et al. 1999; Häfner 2004; Buckley und Stahl 2007). Eine Vielzahl neurobiologischer Auffälligkeiten, wie zunehmender Verlust der grauen Hirnsubstanz, eine verminderte Integrität der weißen Hirnsubstanz und funktionale frontale Hypofunktion, gehen mit einer gravierenden Abnahme kognitiver Fähigkeiten (z.B. Gedächtnis, Aufmerksamkeit und Exekutivfunktionen) einher (Weinberger et al. 2001; DeLisi 2008; Kubicki et al. 2009).

\subsubsection{Epidemiologie}

Die Inzidenz der Schizophrenie liegt unter strengen und international vergleichbaren Kriterien über alle Länder, Klimazonen und Kulturen hinweg bei 1\% (Jablensky et al. 1992). In einer Metaanalyse von McGrath et al. (2008) wurde eine Punktprävalenz zwischen 0,2\% und 1,0\% ermittelt. Das Risiko, einmal im Leben an einer Schizophrenie zu erkranken, liegt bei 0,45\% in beiden Geschlechtern (McGrath et al. 2008). Eine im Vergleich zu psychisch Gesunden um 2bis 3-fach erhöhte Mortalität unter Betroffenen kann auf eine erhöhte Suizidrate, besonders aber auf einen schlechteren physischen Gesundheitszustand zurückgeführt werden (Joukamaa et al. 2006; Saha et al. 2007).

\subsection{2 Ätiopathogenese}

Die Erblichkeit der Schizophrenie liegt bei ca. 80\%. Verwandte ersten Grades haben im Vergleich zu der generellen Bevölkerung ein 5- bis 10-fach erhöhtes Risiko, die Erkrankung zu entwickeln (Cardno und Gottesman 2000). Die Heritabilität von 48\% bei eineiigen und 17\% bei zweieiigen Zwillingen spricht jedoch gegen eine typisch mendelsche Vererbung (Gottes- 
man 1991). Mutationen in bestimmten Genen (wie z.B. DISC1 und NRG1), die neuronales Wachstum, Neurotransmission und/oder die neuronale Zellfunktion ursächlich beeinflussen, konnten in Studien mit der Erkrankungswahrscheinlichkeit assoziiert werden (Harrison und Law 2006; Pearlson und Folley 2008; Brandon et al. 2009). Obwohl es möglich ist, dass die Schizophrenie durch diese seltenen individuellen Mutationen verursacht ist, treten diese auch ohne Krankheitswert in der generellen Bevölkerung auf. Zudem ist keine dieser Mutationen notwendig noch allein verantwortlich für den Ausbruch der Erkrankung (Pearlson und Folley 2008). So ist unklar, wie stark ein sog. Kandidatengen für den Ausbruch der Erkrankung verantwortlich gemacht werden kann, da letztlich keines definitiv genetisch mit der Schizophrenie gekoppelt ist (Norton et al. 2006). Neue Erkenntnisse genetischer Erkrankungsgrundlagen erhofft man sich aus genomweiten Assoziationsstudien. Insgesamt geht man von einer multifaktoriell bedingten Erkrankung aus. Demnach müssen für den Ausbruch der Erkrankung mehrere Teilursachen vorliegen. Ein Erklärungskonzept ist die „Two Hit Hypothesis“ (Bayer et al. 1999). Demnach führen vererbte oder spontan mutierte genetische Defekte in einem oder mehreren spezifischen Kandidatengenen zu einer veränderten Hirnentwicklung-, reifung und Funktionsweise (erster „hit"). Der zweite „hit“ beinhaltet die Einwirkung negativer Umwelteinflüsse. Solche Umweltfaktoren sind von unterschiedlicher Qualität und umfassen z. B. Schwangerschafts- oder Geburtskomplikation, virale Infektion, soziale Stressfaktoren, Schädel-Hirn-Trauma oder Cannabiskonsum (Jones et al. 1998; Pearce 2001; Nielsen et al. 2002; D'Souza et al. 2005; Krabbendam und van Os 2005). So geht man davon aus, dass äußere Einflüsse die pathogene Funktion der Kandidaten-Gene induzieren und folglich den Ausbruch der Schizophrenie verursachen.

\subsubsection{Symptomatologie}

Die Kernsymptome der Schizophrenie spiegeln sich in andauernder Positivsymptomatik, dem ausgeprägten Verlust bereits ausgebildeter kognitiver Fähigkeiten und einer über die Zeit persistierenden Negativsymptomatik wider. Begleitend treten Zeichen allgemeiner Psychopathologie auf, wie z.B. Depressivität, Angst und Angespanntheit (Andreasen 1995; Horan und Blanchard 2003). Keines dieser Zeichen ist pathognomonisch; zwar tritt jedes Zeichen in der Gruppe der Erkrankten auf, jedoch variiert die individuelle Art und Intensität der Symptomatologie erheblich.

\subsubsection{Die Positivsymptomatik}

Die Positivsymptomatik bezeichnet ein „Mehr“ des generellen Erlebens. Dazu werden Halluzinationen, Wahnideen (inhaltliche Denkstörungen), psychomotorische Erregung, Störungen 
des Denkablaufs (formale Denkstörungen) und Ich-Störungen gezählt (Andreasen und Olsen 1982; Scharfetter 1996).

Die häufigsten Symptome sind akustische Halluzinationen (kommentierende, imperative, dialogische, beschimpfende Stimmen) und Wahnvorstellungen (z.B. Verfolgungs-/ Beeinträchtigungs-, Beziehungs-, Schuld- und Größenwahn). Inhaltliche Denkstörungen üben einen starken Effekt auf die Emotionen und das Verhalten aus, jedoch ist die Positivsymptomatik nicht mit kognitiven Leistungsbeeinträchtigungen assoziiert (Dominguez Mde et al. 2009). IchStörungen beschreiben das Phänomen einer Aufweichung der Ich-Umwelt-Grenze. Zum einen kann sich die Person von sich selbst entfremdet, zum anderen durch die Umwelt beeinflusst fühlen. Häufig schildern Schizophrene ein von außen manipuliertes, fremdbeeinflusstes Denken (Scharfetter 1996).

\subsubsection{Die Negativsymptomatik}

Die Negativsymptomatik beschreibt ein „Weniger“ des ursprünglichen Erlebens. Sie findet in Antriebslosigkeit, emotionalem Rückzug, sozialer Passivität, flachem Affekt, mangelnder Spontaneität und Kreativität, reduzierter Willenskraft, stereotypen Gedanken, Sprachverarmung und motorischen Defiziten Ausdruck (Crow 1980; Häfner 2004). Diese „Defizitsymptome“ sind größtenteils schon vor dem ersten, von einer Positivsymptomatik geprägten, Ausbruch der Erkrankung vorhanden und persistieren oder verstärken sich über den weiteren Erkrankungsverlauf (Harvey et al. 2006). Die Negativsymptomatik ist stark mit kognitiven Defiziten assoziiert und wird daher als Mediator von Kognition und allgemeinem Funktionsniveau verstanden (Ventura et al. 2009). Die daraus resultierenden Einschränkungen im sozialen Umfeld und Arbeitsalltag üben langfristig einen wesentlich größeren Leidensdruck aus als die Positivsymptomatik (Milev et al. 2005). Deshalb ist die Negativsymptomatik ein wesentlich wichtigerer Ansatzpunkt für Therapien. Trotz intensiver Forschung ist es jedoch bislang nicht gelungen, die Negativsymptomatik medikamentös erfolgreich zu behandeln (Leucht et al. 2009 a).

\subsubsection{Die Kognition}

Bereits 1919 stellte Emil Kraepelin unter der Bezeichnung „Dementia Praecox“ (vorzeitige Demenz) für die heute als Schizophrenie bezeichnete Erkrankung die Abnahme der kognitiven Fähigkeiten als wesentliches Krankheitsmerkmal heraus (Kraepelin 1919). Seit einigen Jahren sind die kognitiven Defizite aus folgenden Gründen wieder in das Zentrum klinischer Forschung gerückt: Sie treten sowohl in der Kindheit als auch im Erwachsenenalter auf, erscheinen mit hoher Regelmäßigkeit, sind relativ stabil über den Krankheitsverlauf hinweg und von psychotischen Symptomen unabhängig (Fioravanti et al. 2005; Dominguez Mde et al. 
2009). 1991 konnten in einer Studie mit 36 unmedizierten Schizophrenen und 36 Kontrollpersonen kognitive Defizite als direktes Symptom der Schizophrenie nachgewiesen werden (Saykin et al. 1991). Am stärksten sind die Bereiche Gedächtnis, Aufmerksamkeit und Exekutivfunktionen betroffen, weniger stark dagegen verbale und visuospatiale Fähigkeiten (Green 1996 b; Heinrichs und Zakzanis 1998). In einer Metaanalyse von Heinrichs und Zakzanis wurden kognitive Kennwerte von 204 Studien mit insgesamt 7420 schizophrenen Patienten und 5865 gesunden Kontrollprobanden untersucht. Unter den Schizophrenen war das durchschnittliche Leistungsniveau 1,5 bis 2 Standardabweichungen geringer als das der gesunden Kontrollpersonen (Heinrichs und Zakzanis 1998; Fioravanti et al. 2005). Diese ausgeprägten Defizite konnten in einer weiteren Studie bei etwa 80\% aller Schizophrenen nachgewiesen werden (Wilk et al. 2004). Wie auch hinsichtlich der Positiv- und Negativsymptomatik imponieren bei jedem Erkrankten unterschiedliche Muster der kognitiven Beeinträchtigung, die keiner Gesetzmäßigkeit folgen (Andreasen 1995). Gezeigt werden konnte jedoch, dass kognitive Funktionseinbußen dem ersten floriden Ausbruch der Schizophrenie mit Positivsymptomatik vorausgehen und bei Ersterkrankung schon sehr stark ausgeprägt sind (ErlenmeyerKimling et al. 2000; Fuller et al. 2002). Besonders der „schulische Leistungsknick“ in der Adoleszens wird als wesentlicher Prädiktor für einen Übergang von unspezifischen Symptomen in das Vollbild der Schizophrenie gedeutet (Reichenberg et al. 2006). Auch wurden als Ursachen für persistierende pathologische ZNS-Prozesse mit kognitivem Leistungsabbau die Dauer der unbehandelten Psychose vor Erstmedikation (DUP), die Länge psychotischer Phasen sowie die Anzahl der Erkrankungsrückfälle mit damit einhergehenden kognitiven Degenerationsschüben diskutiert (Breier et al. 1991; Wyatt 1991; Hoffman und McGlashan 1993; Perkins et al. 2005). Für eine genetische Prädisposition der Schizophrenie mit einhergehender kognitiver Beeinträchtigung spricht die Tatsache, dass nicht erkrankte Verwandte schizophrener Patienten in Studien ebenfalls kognitive Beeinträchtigung zeigen, welche jedoch moderater ausfallen als bei den Betroffenen (Cannon et al. 1994; Faraone et al. 1996; Toomey et al. 1998). Auf den entscheidendsten Aspekt der kognitiven Beeinträchtigungen machte Green 1996 aufmerksam. Er belegte, dass die Alltagsbewältigung, der Neu- bzw. Wiedererwerb von Fähigkeiten und somit die Rehabilitation im gesellschaftlichen und beruflichen Leben Schizophrener ursächlich vom kognitiven Leistungsniveau abhängen (Green 1996 a; Green et al. 2004). Somit steht die Verbesserung der Kognition als ein bedeutsames Behandlungsziel im Forschungsfokus und wird als diagnostisches Kriterium zukünftiger Klassifikationssysteme diskutiert (Keefe und Fenton 2007).

Das National Institute of Mental Health (NIMH) hat das Forschungsprojekt MATRICS (Measurement and Treatment Research to Improve Cognition in Schizophrenia) initiiert, um einheitliche standardisierte kognitive Testbatterien zu entwerfen, kognitive Einschränkungen 
zu analysieren, industriell finanzierte Studien zu evaluieren, auf neue pharmakologische Therapieansätze aufmerksam zu machen und diese zu fördern (www.matrics.ucla.edu 2010). Im Rahmen dieses Forschungsprojektes konnte auch nachgewiesen werden, dass die von der Industrie als kognitionsfördernd eingeführten Antipsychotika der zweiten Generation nicht mit Verbesserungen von Kognition assoziiert sind (Keefe et al. 2007).

Ob das schon zu Beginn der Schizophrenie deutliche kognitive Defizit in Arbeitsgedächtnis, Aufmerksamkeit und Verarbeitungsgeschwindigkeit über die Erkrankungsdauer stabil bleibt oder ein Entwicklungsdefizit darstellt, ist noch nicht endgültig geklärt. Die Tatsache, dass die Erkrankung einiger Schizophrener nach dem Ausbruch vollständig remittiert, bei anderen Patienten jedoch chronifiziert und im Alter eine starke zusätzliche Abnahme kognitiver Leistungen zeigt (Kirkpatrick et al. 2008), verdeutlicht die Heterogenität und die Problematik dieser Fragestellung.

In einigen Längsschnittstudien und Metaanalysen konnte gezeigt werden, dass die zu Beginn der Erkrankung starke Abnahme des kognitiven Funktionsniveaus nicht über den Erkrankungsverlauf zunahm (Rund 1998; Hoff et al. 2005; Kurtz 2005) und die Differenz kognitiver Leistung zwischen Gesunden und Schizophrenen über den Erkrankungsverlauf stabil blieb (Bowie et al. 2008). Jedoch konnten Oie et al. in einer Studie, die auch sehr früh erkrankte Personen einschloss, über einen 13-jährigen Zeitraum eine signifikante Abnahme oder eine fehlende Weiterentwicklung in den kognitiven Domänen Wortgedächtnis, Aufmerksamkeit und Informationsverarbeitungsgeschwindigkeit im Vergleich zu gesunden Kontrollpersonen nachweisen (Oie et al. 2010). Inzwischen wird die Ursache kognitiver Beeinträchtigung im Synergismus von kognitivem Entwicklungsdefizit und im Vergleich zu Gesunden geringerer kognitiver Reifung vermutet (Reichenberg et al. 2010). Insgesamt erzielen gesunde sowie schizophrene Menschen im Alter schlechtere kognitive Leistungen in den Domänen Gedächtnis, schlussfolgerndem Denken, Exekutivfunktionen und Wortschatz (Kern et al. 2008; Harvey et al. 2010; Salthouse 2010).

Die neuropsychologische Testung ist auch deshalb von hohem wissenschaftlichen Interesse, da bekannt ist, dass frontotemporale Dysfunktion und eine Abnahme der grauen Hirnsubstanz im präfrontalen Kortex, anterioren Cingulum, Hippocampus und superioren temporalen Gyrus mit Defiziten in den Bereichen Gedächtnis und Exekutivfunktionen assoziiert sind (Sanfilipo et al. 2002; Perlstein et al. 2003; Antonova et al. 2004; Rusch et al. 2007). So offenbaren sich hirnmorphologische Veränderungen als kognitive Defizite. Gehirnatrophie, Vergrößerung der Ventrikel und multifokale Strukturveränderungen des ZNS Schizophrener konnten sowohl in in-vivo- als auch in post-mortem-Studien nachgewiesen werden (Harrison et al. 2003; DeLisi 2008). Rapoport et al. fanden eine jährliche Reduktion des Thalamus von $7 \%$ sowie progressive Vergrößerungen der Ventrikel von 10\% pro Jahr (Rapoport et al. 1997). 
Im Vergleich zu gleichaltrigen Gesunden zeigten Schizophrene ein geringeres Volumen des Hippocampus. Diese Differenzen waren bei älteren Patienten größer als bei jüngeren (Chakos et al. 2005). Über eine Dauer von 20 Jahren war der progressive Verlust von Hirnsubstanz bei chronisch erkrankten Patienten doppelt so groß wie bei gleichaltrigen Kontrollpersonen (Hulshoff Pol und Kahn 2008). Dementsprechend konnten Harvey et al. bei über 65-jährigen Schizophrenen einen größeren Verlust kognitiver Fähigkeiten feststellen als bei gesunden Kontrollpersonen (Harvey et al. 1996). Als Korrelat auf zellulärer Ebene werden Stoffwechselstörungen der Neurone mit einhergehendem Verlust von Axonen, einer geringeren Bildung von Synapsen und dadurch verminderter Neuroplastizität diskutiert (McGlashan und Hoffman 2000; Lieberman et al. 2001; Mirnics et al. 2001). Ausgehend von den hier dargestellten Befunden darf man von neurodegenerativen Prozessen ausgehen, die besonders im Vorfeld oder zu Beginn der Schizophrenie stattfinden.

\subsubsection{Der Krankheitsverlauf}

Die Schizophrenie entwickelt sich am häufigsten in der Adoleszenz oder im jungen Erwachsenenalter. Sie äußert sich in Einbußen kognitiver Fähigkeiten, sozialer Isolation, Interessenverlust und extremen Stimmungsschwankungen (Häfner 2004). Diese Symptome können bereits als „Vorboten“ der Erkrankung auftreten und verschlechtern sich vor allem innerhalb der ersten 5 bis 10 Erkrankungsjahre. Anschließend stabilisieren sie sich auf einem im Vergleich zum Erkrankungsausbruch schlechteren Niveau (Lieberman et al. 2001). Obwohl die Mehrheit der Patienten im Erkrankungsverlauf diese Merkmale der Degeneration aufweist, zeigen sich deutliche inter- und intraindividuelle Unterschiede, sowohl in der Qualität, als auch der Quantität und Dauer der Symptome. In diesem Spektrum stehen remittierte Patienten mit leichter Residualsymptomatik schwerkranken chronischen Patienten mit extremer Negativsymptomatik und gravierenden kognitiven Einbußen gegenüber. Aufgrund der Vielfältigkeit der Verläufe kommen große Untersuchungen zu unterschiedlichen Ergebnissen. Menezes et al. konnten innerhalb des ersten Erkrankungsjahres bei $42 \%$ der Patienten ein Wiedererlangen des ursprünglichen Funktionsniveaus feststellen; 52\% zeigten bleibende Einbußen, davon die Hälfte massiver Art (Menezes et al. 2006). Eine Übersichtsarbeit über Langzeitverläufe der Schizophrenie legt nahe, dass der Verlauf dreigeteilt ist: in milde, moderate und schwere Verläufe (Jobe und Harrow 2005).

\subsubsection{Die Klassifikation}

Obwohl die Erkrankung Schizophrenie mit deutlichen Veränderungen z.B. neuronaler Strukturen und der Dopamintransmission assoziiert ist, existieren bislang weder Laboranalysen oder bildgebende Verfahren noch psychologische Tests, die sich als pathognomonisch für die 
Schizophrenie erweisen konnten (Lewis et al. 2005). Deshalb wird die Erkrankung rein deskriptiv auf der Basis des klinischen Syndroms diagnostiziert. Um eine diagnostische Reliabilität zu gewährleisten, sind diese charakteristischen Merkmale in spezifischen Kriterien der beiden international anerkannten operationalen Klassifikationssysteme ICD-10 (Dilling und Freyberger 2006) und DSM-IV-TR (AmericanPsychiatricAssociation 2000) formuliert worden. Diese schlagen eine Einteilung in verschiedene Subtypen vor, z.B. paranoidhalluzinatorische, hebephrene oder katatone Schizophrenie. Aufgrund weitgehender Übereinstimmungen zwischen beiden Klassifikationssystemen soll im Folgenden nur die in der Untersuchung angewandte Klassifikation nach DSM-IV-TR näher erörtert werden. Demnach müssen erstens mindesten 2 charakteristische Symptome wie Wahn, Halluzinationen, Denkstörungen, desorganisiertes bzw. katatones Verhalten oder Negativsymptome für mindestens einen Monat vorliegen. Das zweite Kriterium umfasst Dysfunktionen in beruflicher oder sozialer Hinsicht, z.B. einen krankheitsbedingten Abstieg in interpersonellen Kontakten, im Arbeitsstatus und/oder bei persönlicher Hygiene. Drittens wird das Zeitkriterium erfasst, das eine Dauer der Beeinträchtigungen von mindestens 6 Monaten verlangt und viertens dürfen die Symptome nicht durch etwaige andere (psychische) Erkrankungen, wie z.B. SubstanzAbhängigkeiten, erklärt werden können (vgl. Tab. 1).

Tabelle 1: Kriterien zur Diagnose der Schizophrenie (nach Diagnostic and Statistical Manual of Mental Disorders: DSM-IV-TR, AmericanPsychiatricAssociation 2000, 312)

\begin{tabular}{|c|l|}
\hline & \multicolumn{1}{|c|}{ Diagnostische Kriterien für Schizophrenie } \\
\hline A & $\begin{array}{l}\text { Charakteristische Symptome } \\
\text { Mindesten zwei der folgenden, jedes für einen erheblichen Teil der Zeitspanne von 1 Monat (oder weni- } \\
\text { 1. Wahn } \\
\text { 2. Halluzinationen } \\
\text { 3. desorganisierte Sprache } \\
\text { 4. grob desorganisiertes oder katatones Verhalten }\end{array}$ \\
\hline B & Sozialberufliche Leistungseinbußen \\
\hline C & $\begin{array}{l}\text { Zeichen des Störungsbildes halten kontinuierlich für mindestens 6 Monate an. Diese 6-monatige Periode } \\
\text { muss mindestens einen Monat mit Symptomen des Kriteriums A beinhalten. }\end{array}$ \\
\hline D & Ausschluss von schizoaffektiver und affektiver Störung \\
\hline E & Ausschluss von Substanzeinfluss und symptomerklärenden Erkrankungen \\
\hline
\end{tabular}




\subsection{Die Therapie der Schizophrenie}

\subsubsection{Die Wirkmechanismen der Antipsychotika}

Im Rahmen der Behandlung der Schizophrenie ist die medikamentöse Behandlung mit Antipsychotika (AP) unverzichtbar. Indem sie im Akutfall die Positivsymptomatik reduzieren, können sie als Schlüssel angesehen werden, der den Patienten die Tür für den Kontakt in die reale Welt eröffnet. Langfristig werden diese Substanzen auch zur Rückfallprophylaxe eingesetzt. Orale, intramuskuläre und intravenöse Applikationsformen stehen zur Verfügung.

Die Grundlage der antipsychotischen Wirkung ist die pharmakologisch antagonistische Wirkung am kortikalen Dopamin $\mathrm{D}_{2}$-Rezeptor $\left(\mathrm{D}_{2}-\mathrm{R}\right)$. Je größer die Affinität eines Wirkstoffs für den $\mathrm{D}_{2}-\mathrm{R}$ ist, desto größer ist dessen antipsychotische Potenz (Kapur und Mamo 2003). Je geringer die neuroleptische Potenz eines (typischen) AP, desto größer ist dessen dämpfende und sedierende Eigenschaft (Leucht et al. 2009 b). Es wird angenommen, dass eine Blockade von $65 \%$ der zentralen $\mathrm{D}_{2}-\mathrm{R}$ zu zufriedenstellender antipsychotischer Wirkung führt. Höhere Raten sind mit ernsthaften Nebenwirkungen verbunden (Tauscher und Kapur 2001). Unterschieden wird zwischen antipsychotischen Medikamenten der ersten Generation (typische Antipsychotika; Typika; first generation antipsychotics, FGA) und denen der zweiten Generation (atypische Antipsychotika; Atypika; second generation antipsychotics, SGA).

Merkmal der 1950 eingeführten FGA ist deren chemische strukturelle Ähnlichkeit und die fast ausschließliche Blockade der $\mathrm{D}_{2}-\mathrm{R}$. Bei guter Wirksamkeit gegen die Positivsymptomatik erzeugen sie jedoch besonders unter hoher Dosierung unterschiedliche extrapyramidalmotorische Störungen (EPMS) als Nebenwirkung (vgl. Seite 26 f.). Diese entstehen, da sich im nigrostriatalen System ebenfalls $\mathrm{D}_{2}-\mathrm{R}$ befinden. Über dopaminerge Projektionen inhibieren diese Rezeptoren die Präzentralregion des Nucleus caudatus, eine zentrale Schaltstelle motorischer Impulse. Bei antipsychotischer Medikation werden auch die nigrostriatalen $\mathrm{D}_{2}-\mathrm{R}$ blockiert, woraus eine „sekundäre Überfunktion“ dieser Hirnregion resultiert, welche sich in EPMS äußert. Dabei handelt es sich zum einen um reversible Erscheinungen (z.B. Frühdyskinesien, Parkinsonoid, Dystonie, Hyperkinese, Akathisie), zum anderen um solche irreversibler Art (tardive Dyskinesien und tardive Akathisie oder Spät-Dyskinesien) (Simpson und Angus 1970). Die genaue Ursache der tardiven Dyskinesien ist bislang jedoch noch nicht bekannt. Das Auftreten der EPMS kann nicht vorhergesagt werden. Reversible EPMS können mit Anticholinergika kupiert werden, eine Behandlungsmöglichkeit der Späterscheinungen besteht hingegen nicht (s.u.).

Die seit ca. 10 Jahren häufig eingesetzten SGA zeigen ebenfalls eine gute Wirkung auf die Positivsymptomatik und führen seltener zu EPMS (Leucht et al. 1999). Diese chemisch sehr heterogene Medikamentengruppe interagiert zusätzlich zum $\mathrm{D}_{2}-\mathrm{R}$ in unterschiedlicher Vertei- 
lung an anderen Rezeptoren des ZNS (z.B. Serotonin-, Acetylcholin-, Noradrenalin- und Histaminrezeptoren). Das Nebenwirkungsspektrum der SGA umfasst unterschiedliche endokrine Störungen (z.B. Leber- und Nierenfunktionsstörungen, kardiovaskuläre Störungen), die zum Teil so schwerwiegend sind, dass eine internistische Behandlung notwendig wird (Conley und Kelly 2005; Patel et al. 2009). Die sehr ernsten Nebenwirkungen der SGA stehen in Verdacht, die erhöhte Mortalität unter Schizophrenen mit zu bedingen (Joukamaa et al. 2006; Saha et al. 2007). Trotz dieser Erkenntnisse empfiehlt die aktuelle Behandlungsleitlinie Schizophrenie (noch) die SGA als Medikation der ersten Wahl (Gaebel und Falkai 2005).

Abgesehen von dem Effekt auf die Positivsymptomatik ist die Wirksamkeit der verfügbaren AP sehr begrenzt. Wegen subjektiv empfundener Ineffizienz und der belastenden Nebenwirkungen betrug die Abbruchrate in einer 18-monatigen Langzeitstudie für FGA und SGA 74\% (Lieberman et al. 2005). Nur 41\% medikamentös behandelter Patienten sprachen nach Ergebnissen einer Metaanalyse von Leucht et al. (2009) auf SGA an, hingegen sprachen 24\% der Behandelten auf Placebo an. Ebenso konnte eine Abnahme der Effektivität der SGA über die Behandlungszeit beschrieben werden (Leucht et al. 2009 a). So wird an der Entwicklung besser wirksamer Substanzen geforscht, die z.B. nikotinerge, cholinerge, dopaminerge und glutamaterge Eigenschaften aufweisen (Buchanan et al. 2007).

Wegen mangelnder Wirksamkeit verfügbarer AP, oder auch um einen besseren oder schnelleren Therapieerfolg zu erreichen, bekommen Patienten in der Praxis immer häufiger mehr als ein Antipsychotikum verschrieben (Polypharmazie) und erreichen sehr hohe antipsychotische Tagesdosen (Kane et al. 2003; Stahl und Grady 2004; Ito et al. 2005). Studien zeigten, dass der Anteil von „polypharmazierten“ Schizophrenen zwischen 1999 und 2005 von 3,3\% auf 13,7\% gestiegen ist und sich dieser Trend verstärkt fortsetzt (Gilmer et al. 2007; Mojtabai und Olfson 2010). Es ist erwiesen, dass Polypharmazie mit sehr hohen antipsychotischen Dosen einhergeht, die die empfohlenen Maximaldosen der Richtlinien übersteigen (Elie et al. 2010). Bislang ist der Nutzen sehr hoher antipsychotischer Dosen in keiner kontrollierten klinischen Studie belegt worden und es ist unklar, ob eine schwerwiegendere Psychopathologie höhere Dosen an AP erfordert (Lipkovich et al. 2008). Behandlungsrichtlinien verschiedener Länder, wie auch die deutsche Behandlungsleitlinie Schizophrenie (Gaebel und Falkai 2005), legen nahe erst dann mit mehr als einem Antipsychotikum gleichzeitig zu behandeln, wenn Monotherapien mit verschiedenen Antipsychotika und auch dem Antipsychotikum Clozapin keinen Behandlungserfolg gezeigt haben (Miller et al. 2004; Gaebel und Falkai 2005). Begründet ist das durch die Studienlage, die verdeutlicht, dass die Verschreibung mehrerer Antipsychotika zu signifikant mehr Nebenwirkungen (Honer et al. 2006), längeren Krankenhausaufenthalten und einer schlechteren Krankheitswahrnehmung Schizophrener führt (Centorrino et al. 2004; Mouaffak et al. 2006). Auf der anderen Seite lieferte eine Metaanalyse empirische Unterstüt- 
zung für die gleichzeitige Applikation mehrerer AP, jedoch nur unter spezifischen Voraussetzungen (Correll et al. 2009). Auf biochemischer Ebene wird als wichtiger Grund für steigende antipsychotische Tagesdosen und antipsychotische Polypharmazie die Abnahme der APWirksamkeit unter langzeitiger, kontinuierlicher antipsychotischer Therapie vermutet. Dem liegt eine erhöhte Expression und zunehmende Sensitivität (Supersensitivität) zentraler $D_{2}-R$ zugrunde, die anhand von Tiermodellen nachgewiesen werden konnten (Seeman P et al. 2005; Samaha et al. 2007). Die nicht durch AP blockierten ,supersensitiven“ $D_{2}-R$ reagieren schneller und stärker auf das ausgeschüttete Dopamin, was sich in einer Zunahme psychopathologischer Symptome und häufigeren Krankheitsrückfällen über die Länge der Medikationsdauer äußert. Jedoch scheinen die antipsychotischen Tagesdosen nicht über die gesamte Erkrankungsdauer zu steigen. Uchida et al. (2008) stellten in einer Untersuchung fest, dass die antipsychotische Tagesdosis über die gesamte Medikationszeit hinweg einer umgekehrten U-Form gleicht. Demnach bekamen Patienten bis zum mittleren Lebensalter steigende antipsychotische Tagesdosen, welche dann zunächst auf einem Plateau stabil blieben, bevor sie im hohen Lebensalter wieder abfielen (Uchida et al. 2008).

Da die Rehabilitation im gesellschaftlichen und beruflichen Leben Schizophrener ursächlich von deren kognitivem Leistungsniveau abhängt (vgl. Seite 3 f.), steht die Wirksamkeit der Antipsychotika auf das kognitive Leistungsniveau im Forschungsfokus (Green 1996 a; Green et al. 2004; Bowie et al. 2006).

\subsubsection{Assoziation von antipsychotischer Behandlung mit Kognition}

In der Vergangenheit wurde eine Vielzahl von Studien veröffentlicht, die einen positiven Effekt vor allem der SGA auf die kognitiven Leistungen nahelegten. Die Verbesserungen des Lernens, der Aufmerksamkeit, der psychomotorischen Geschwindigkeit und der verbalen Flüssigkeit unter Therapie mit SGA wurden in Einzelstudien und Metaanalysen belegt (Harvey und Keefe 2001; Woodward et al. 2005). Dem gegenüber standen Untersuchungsergebnisse mit niedrig dosierten FGA, welche einen vergleichbar positiven Effekt auf die Kognition verdeutlichten. Etwaige Wirksamkeitsunterschiede der beiden AP-Klassen wurden auf v.a. Dosisunterschiede, Komedikation und Nebenwirkungen zurückgeführt (Geddes et al. 2000; Mishara und Goldberg 2004; Weickert und Goldberg 2005). Die Annahme, dass beide Medikationstypen vergleichbare Effekte auf das kognitive Leistungsniveau Ersterkrankter sowie chronischer Patienten zeigen, wurde in neueren Studien bestätigt (Keefe et al. 2007; Swartz et al. 2008; Davidson et al. 2009; Leucht et al. 2009 a/b). Fest steht, dass sowohl die kognitiven Leistungen als auch das Ansprechen auf AP genetisch determiniert sind (Kane et al. 2008). Allerdings wird inzwischen vermutet, dass die in Studien nachgewiesene kognitive Verbesserung unter medikamentöser Therapie größtenteils auf Placebo- und Übungseffekte zurückzu- 
führen ist und demnach nicht in erster Linie den AP zugeschrieben werden kann (Goldberg et al. 2010). Gegenteilig konnte beispielsweise gezeigt werden, dass hohe antipsychotische Tagesdosen (>1000 CPÄ/d) mit signifikant schlechteren Ergebnissen in den kognitiven Einheiten visuelles Gedächtnis, Wortgedächtnis, Exekutivfunktionen und praktischer IQ assoziiert sind, welche nicht durch die Krankheitsschwere erklärt werden können (Hori et al. 2006; Elie et al. 2010). Im Hinblick auf kognitive Defizite bei Schizophrenen werden medikamentöse Nebenwirkungen wie Sedierung und motorische Nebenwirkungen (McEvoy et al. 1991; Tauscher und Kapur 2001), sowie direkt von AP ausgehende schädliche Effekte diskutiert. So verwies eine kleine Interventionsstudie darauf, dass eine Dosisreduktion bei Schizophrenen mit hoher antipsychotischer Tagesdosis zu einer Verbesserung kognitiver Leistungen führen kann (Kawai et al. 2006). Hoff et al. konnten zeigen, dass das SGA Clozapin das Arbeitsgedächtnis und weitere Exekutivfunktionen beeinträchtigt (Hoff et al. 1996). Da es ethisch nicht zu verantworten ist, Langzeitstudien mit unmedizierten Schizophrenen oder sehr hohen antipsychotischen Tagesdosen durchzuführen, liegen keine eindeutigen Daten vor, anhand derer man die Effekte der AP auf Kognition, Krankheitsverlauf, zentrale morphologische Prozesse und Komorbiditäten hinreichend erklären kann. Es liegen jedoch Ergebnisse aus Tierexperimenten vor. So verbesserten geringe Dosen von Clozapin durch ein Pharmakon induzierte Defizite des Arbeitsgedächtnisses bei Affen. Dem Menschen vergleichbare therapeutische Dosen bewirkten jedoch in dem Experiment einen gegenteiligen Effekt (Murphy et al. 1997). Darüber hinaus konnten Störungen des Arbeitsgedächtnisses unter Clozapin, Haloperidol und Risperidon bei Ratten beobachtet werden (Didriksen 1995; Addy und Levin 2002). Auch Skarsfeldt konnte unter Medikation von Clozapin, Olanzapin, Ziprasidon, Haloperidol und Risperidon beeinträchtigte Gedächtnisleistungen bei jungen Ratten feststellen (Skarsfeldt 1996).

\subsubsection{Die Begleitmedikation}

\subsubsection{Anticholinerge Medikation}

Auf den Seiten 7 f. wurde beschrieben, dass die antipsychotische Wirkung der Antipsychotika hauptsächlich durch die Blockade von $\mathrm{D}_{2}-\mathrm{R}$ im Kortex vermittelt wird, jedoch die gleichzeitige Blockade nigrostriataler $\mathrm{D}_{2}-\mathrm{R}$ zu einer sekundären Überfunktion in Teilen des Striatums führt, die sich klinisch in EMPS manifestiert. Deshalb wird häufig die Augmentation anticholinerger Substanzen notwendig. Die sog. Anticholinergika wirken hemmend auf cholinerge Interneurone, die ebenfalls erregend auf das Striatum wirken. So wird versucht, die reversiblen EPMS zu kupieren. Es ist jedoch bekannt, dass diese Substanzen Lern-, Gedächtnis- und In- 
terferenzleistungen bei schizophrenen Patienten beeinträchtigen (Spohn und Strauss 1989; Minzenberg et al. 2004; Vinogradov et al. 2009).

\subsubsection{Benzodiazepine}

Oft werden Benzodiazepine als Bedarfs- oder Regelmedikation bei Schizophrenen eingesetzt, da diese gute Effekte bei Angst und Spannungszuständen zeigen. Allerdings führt die sedierende und hypnotische Wirkung der Benzodiazepine zu einer Beeinträchtigung der kognitiven Leistungsfähigkeit (Kleinknecht und Donaldson 1975; Hinrichs et al. 1982). In einer Metaanalyse konnten negative Effekte nach dauerhafter Benzodiazepinapplikation in den Bereichen Wortgedächtnis, Informationsverarbeitungsgeschwindigkeit, Motorik, Arbeitsgedächtnis und genereller Intelligenz (IQ) nachgewiesen werden (Barker et al. 2004).

Zusammenfassend ist schwer zu verifizieren, ob eine vorhandene Negativsymptomatik mit kognitiver Beeinträchtigung allein durch das Krankheitsbild „Schizophrenie“ oder durch die applizierte Medikation mitbegründet ist. Vorstellbar ist, dass ein synergistischer Effekt beider Faktoren zu dem klinischen Gesamtbild führt.

\subsubsection{Nichtmedikamentöse Behandlungsansätze}

Da die antipsychotische Therapie zum einen mit deutlichen Nebenwirkungen einhergeht und zum anderen etwaige direkte negative Effekte auf das kognitive Leistungsniveau nicht auszuschließen sind, rücken nichtmedikamentöse Therapieverfahren vermehrt in das Zentrum der Aufmerksamkeit. Zum Beispiel ist die Cognitive Enhancement Therapy (CET) eine Therapieform, die kognitive und soziale Rehabilitationsansätze integriert. In einer 2-jährigen Studie konnte nachgewiesen werden, dass die CET sowohl einen positiven Effekt auf die Kognition hat, als auch zu einer verminderten Abnahme der grauen Hirnsubstanz führt (Eack et al. 2010). Ebenso konnte eine Metaanalyse zeigen, dass die Förderung kognitiver Leistungen zu einer Verbesserung um durchschnittlich 0,4 SD des kognitiven und des gesamten funktionellen Leistungsniveaus führen kann (McGurk et al. 2007).

Den vorherigen Kapiteln ist zu entnehmen, dass Schizophrene große interindividuelle Unterschiede im Hinblick auf Symptomatik, Verlauf und Therapierbarkeit aufweisen. Ein zusätzlich bedeutender Unterschied besteht zwischen Männern und Frauen. Darauf soll im nächsten Kapitel eingegangen werden.

\subsection{Geschlecht und Schizophrenie}

Bereits in der pränatalen Phase wird der Geschlechtsdimorphismus in neuronaler Proliferation und Hirnlateralisation, sowie der körperliche Phänotyp durch Östrogen wesentlich mitbe- 
stimmt. In der Schizophrenie spiegeln sich die geschlechtsspezifischen Unterschiede im Erkrankungsbild, Funktionsniveau, Ansprechen auf Antipsychotika und deren Nebenwirkungsprofil wider.

\subsubsection{Geschlechtsspezifischer Verlauf der Schizophrenie}

Östrogen bewirkt durch eine frühere neuronale Vernetzung, axonale Myelinisierung und Hirnlateralisierung eine frühere Hirnreifung bei Frauen als bei Männern (Taylor 1969). Dadurch sind Männer vulnerabler für frühe Hirnschädigungen, die zu einem früheren Ausbruch der Erkrankung und einem anderen Erkrankungsbild führen. Im Vergleich zu Frauen zeigen Männer einen früheren Krankheitsbeginn mit einem Haupterkrankungsalter zwischen dem 15. und 25. Lebensjahr. Frauen hingegen haben 3 Haupterkrankungsphasen, wobei die erste zwischen dem 15. und 30. Lebensjahr, die zweite zwischen dem 45. und 49. Lebensjahr und die dritte um das 65. Lebensjahr liegt (Castle und Murray 1993; Hafner et al. 1998). Abhängig von Diagnosekriterien und dem Einschlussalter in Studien, haben Männer insgesamt eine um den Faktor 1,4 höhere Inzidenz (McGrath et al. 2004). Diese Tatsache kann zum Teil dadurch erklärt werden, dass Männer aufgrund einer geringeren Medikamentencompliance höhere Rückfallraten zeigen und eine durchschnittlich schwerwiegendere Psychopathologie präsentieren (Diaz et al. 2004). Insgesamt verläuft die Erkrankung bei Frauen günstiger, was z.B. durch kürzere stationäre Krankenhausaufenthalte und ein geringeres Risiko der Rehospitalisierung nach dem ersten stationär-psychiatrischen Krankenhausaufenthalt zum Ausdruck kommt (Angermeyer et al. 1990). Beide Geschlechter zeigen quantitativ gleich viel Positivsymptomatik; schizophrene Männer entwickeln eine stärkere Negativsymptomatik, wohingegen Frauen mehr affektive Symptome, atypische Psychosen, paranoide und zyklisch psychotische Symptome präsentieren (Ring et al. 1991; Szymanski et al. 1995; Schultz et al. 1997). Da Frauen in Zyklusphasen hoher Serum-Östrogenspiegel eine im Vergleich mildere Symptomatik der Schizophrenie darbieten, geht man von einem ,antipsychotischen“ und neuroprotektiven Effekt des Östrogens aus (vgl. Seite 14 f.).

Die Erfassung von Geschlechtsunterschieden bei Schizophrenen ist dadurch erschwert, dass in fast allen klinischen Studien Männer im Vergleich zu Frauen überrepräsentiert sind (Chaves und Seeman 2006). Dies ist zum einen durch die höhere Inzidenz bei Männern bedingt. Zum anderen werden klinische Untersuchungen bevorzugt mit Männern durchgeführt, da etwaige Untersuchungsgrößen nicht auf zyklische Hormonschwankungen zurückzuführen sind. Seeman MV (2004) machte darauf aufmerksam, dass Frauen insgesamt eine bessere Anbindung an Studien zeigen, sodass der Anteil schwer erkrankter schizophrener Frauen im Vergleich zu gleich stark betroffenen Männern größer ist. Dadurch kann ein Auseinanderweichen von In- 
zidenz und Prävalenz der Symptome entstehen, was als geschlechtsspezifisch interpretiert werden kann (Häfner 2004; Seeman MV 2004).

Nach den geschlechtsspezifischen Unterschieden im Verlauf der Schizophrenie, soll nachfolgend der Unterschied kognitiver Leistungen zwischen den Geschlechtern erörtert werden.

\subsubsection{Geschlechtsspezifische Unterschiede in der Kognition}

Im Vergleich zu Frauen bedingt der relativ frühere Ausbruch der Schizophrenie bei Männern eine frühere Unterbrechung der kognitiven und sozialen Reifung, welche zu einem durchschnittlich schlechteren (prämorbiden) kognitiven Leistungsniveau bei Männern führt (Larsen et al. 1996). Die hemisphärische Organisation der kognitiven Leistungen weist eine stärkere Lateralisierung bei Männern auf (McGlone 1978), was sich in einer höheren Effizienz der dominanten linken Hemisphärenfunktion bei Frauen und der nichtdominanten Hemisphärenfunktion bei Männern äußert. So zeigen Frauen z. B. frühere und bessere sprachliche Fähigkeiten auf, die zum größeren Teil auf der dominanten Hemisphäre lokalisiert sind, wohingegen Männer z.B. bessere visuelle Fähigkeiten aufweisen, die zum größeren Teil auf der nichtdominanten Hemisphäre lokalisiert sind (Leung und Chue 2000; Rubin et al. 2008). Da bei Männern die kognitiven Funktionen stärker lateralisiert sind als bei Frauen, geht man davon aus, dass durch die Schizophrenie verursachte Dysfunktionen frontotemporaler Regionen bei Männern weniger effizient kompensiert werden können als bei Frauen. Diese Annahme wird als Ursache einer breiteren kognitiven Beeinträchtigung erkrankter Männer gegenüber erkrankten Frauen vermutet (Flor-Henry 1990; Longenecker et al. 2010). Elsabagh et al. (2009) konnten zeigen, dass bei Männern über die Erkrankungsdauer stärkere Dysfunktion innerhalb des neuronalen Netzwerks des Arbeitsgedächtnisses entstehen als bei Frauen. Auch machte die Arbeitsgruppe darauf aufmerksam, dass die zentralen geschlechtsspezifischen Funktionsunterschiede über die Erkrankungsdauer abnehmen (Elsabagh et al. 2009). Die regulatorischen Eigenschaften von Östrogen auf die Neurotransmission führen sowohl zu kognitiven Geschlechtsunterschieden, als auch zu inter- und intraindividuellen Unterschieden im Verlauf der Schizophrenie bei Frauen. In Zyklusphasen hohen Östrogens erreichten Frauen bessere Leistung im Test des verbalen Arbeitsgedächtnisses; ebenso zeigten postmenopausalen Frauen unter Östrogen-Substitutionstherapie bessere globale kognitive Leistungen als nichtsubstituierte (Rosenberg und Park 2002; Zec und Trivedi 2002). Männer zeigten im Vergleich zu Frauen signifikant schlechtere Ergebnisse in Aufmerksamkeitstests sowie in Bereichen des verbalen Gedächtnisses und der Exekutivfunktionen (Goldstein et al. 1998). 


\subsubsection{Geschlechtsspezifische Unterschiede in der antipsychotischen Therapie}

Männer und Frauen unterscheiden sich sowohl im Verlauf der Schizophrenie, als auch in der Präsentation von Symptomen. Im Folgenden sollen Ursachen erläutert werden, die ebenfalls Unterschiede in der antipsychotischen Therapie zwischen den Geschlechtern bedingen. So verfügen Männer aufgrund ihrer körperlichen Konstitution über ein größeres Verteilungsvolumen für AP, sodass Frauen bei gleicher pro Kopf Dosierung mehr Psychopharmakon pro Kilogramm Körpergewicht einnehmen als Männer. Hingegen verfügen Frauen über relativ mehr Körperfett, in das die lipophilen AP nach Applikation diffundieren (Seeman MV 2004). Mit abnehmendem Plasmaspiegel des AP wird dieses wieder aus dem Fettkompartiment in den Blutkreislauf zurückgegeben und führt so zu gleichmäßigeren AP-Plasmaspiegeln bei Frauen (Pollock 1997). Auf der anderen Seite können in dem relativ größeren Fettkompartiment der Frauen größere Mengen an AP kumulieren, was zu einer höheren Rate unerwünschter Arzneimittelwirkungen führen kann (Seeman MV 2004). Trotz Korrektur für das Körpergewicht konnte gezeigt werden, dass junge Frauen im Durchschnitt eine geringere antipsychotische Tagesdosis einnahmen als Männer (Andia et al. 1995). Allerdings konnten in einer anderen Studie keine signifikanten Unterschiede in der antipsychotischen Tagesdosis zwischen den Geschlechtern festgestellt werden, was auf die Berücksichtigung von Dauer der unbehandelten Psychose, Dauer antipsychotischer Medikation, Typus der AP, Erkrankungsschwere und Dosis pro Körpergewicht zurückgeführt wurde (Pinals et al. 1996). Demgegenüber konnten Usall et al. (2007) in einer Studie an über 10.000 ambulant behandelten Patienten in 10 europäischen Ländern nachweisen, dass Frauen schneller und im größeren Maß auf Antipsychotika ansprachen als Männer (Usall et al. 2007). Dies galt sowohl bei Erst- als auch bei Wiedererkrankung (Szymanski et al. 1995; Robinson et al. 1999 b). Als Ursache dieser größeren Sensitivität der Frauen auf AP wurden verschiedene Einflussgrößen diskutiert. So wird angenommen, dass aufgrund einer geringeren Aktivität AP verstoffwechselnder Cytochrom P 450Enzyme $\left(\mathrm{CYP}_{450}\right)$, Frauen bei gleicher Dosierung höhere Plasmaspiegel von Risperidon, Quetiapin, Olanzapin und Clozapin aufweisen als Männer (Aichhorn et al. 2006; Aichhorn et al. 2007; Leon et al. 2007; Citrome et al. 2009). Stimmig dazu ist die Beobachtung höherer Serumspiegel von Prolaktin (Prl) und Homovanillinsäure (HVA) in Reaktion auf AP bei Frauen im Vergleich zu Männern (Sumiyoshi et al. 1997; Seeman MV 2009). Hohe HVA- und PrlSpiegel im Serum können als Parameter für einen gesteigerten zerebralen Dopaminumsatz gedeutet werden (Amin et al. 1998). Das bedeutet, dass bei gleicher Dosierung des AP eine höhere $\mathrm{D}_{2}-\mathrm{R}$ Besetzung bei Frauen erreicht werden muss und diese somit in größerem Maße auf AP reagieren als Männer. Da Rauchen ebenfalls eine Induktion einiger $\mathrm{CYP}_{450}$ verursachen 
kann (vgl. Seite 16 f.) und der Anteil an Rauchern unter Männern größer ist als unter Frauen (Tang et al. 2007), führt dieser Aspekt zu einer weiteren Schwierigkeit des direkten Geschlechtsvergleiches antipsychotischer Therapie.

Östrogen scheint einen direkten Effekt auf die Geschlechtsunterschiede in antipsychotischer Sensitivität zu haben. Im Tiermodell konnte gezeigt werden, dass Östrogen direkt mit der Dopamintransmission assoziiert ist (Bosse und DiPaolo 1996; Sanchez et al. 2010). Zusätzlich moduliert Östrogen die zentrale Serotonin-, Glutamat- und GABA-Transmission, was als mögliche Ursache neuroprotektiver Eigenschaften diskutiert wird (Brann et al. 2007). Diese Tatsache erklärt, weshalb Phasen geringer Serum-Östrogenspiegel (post-partum, prämenstruell und postmenopausal) mit einem höheren Risiko einer Psychose, einer höheren Rate an Erkrankungsrückfällen und einer schwereren Ausprägung der Psychopathologie assoziiert sind (Riecher-Rossler et al. 1994; Hafner 2003). Daraus wurde geschlossen, dass Östrogen selbst „antipsychotische“ Eigenschaften besitzt und Frauen deshalb im Durchschnitt weniger AP benötigen als Männer. Diese Annahme wurde im Rahmen eines Experimentes bestätigt, in dem Östrogen zusätzlich zu dem AP Haloperidol verabreicht wurde und dadurch eine stärkere Besserung der Psychopathologie nachgewiesen werden konnte (Kulkarni et al. 1996). Auch der geschlechtsspezifische Verlauf der Schizophrenie mit unterschiedlichen Ersterkrankungsaltern und unterschiedlicher Altersverteilung ist mit antipsychotischer Dosierung assoziiert. Zusätzlich scheint bei Frauen im Alter eine relativ größere Reduktion des cerebralen Blutflusses und eine langsamere Abnahme zentraler $\mathrm{D}_{2}-\mathrm{R}$ als bei Männern zu unterschiedlichen Ansprechraten auf AP zu führen (Gur und Gur 1990; Pohjalainen et al. 1998). Schließlich werden geschlechtsspezifische Verlaufs- und Erkrankunsformen der Schizophrenie (vgl. Seite 12 f.) als Ursache unterschiedlichen antipsychotischen Dosierungsverhaltens der betreuenden Psychiater vermutet (Ito et al. 2005).

\subsubsection{Geschlechtsunterschiede in den Nebenwirkungen der Antipsychotika}

Frauen sind hinsichtlich metabolisch-endokriner, kardiovaskulärer und einiger neurologischer Nebenwirkungen stärker betroffen als Männer (Casey 1991; Seeman MV 2009). Spekuliert wird, dass die Ursache in einer im Vergleich zu Männern zu hohen antipsychotischen Dosierung liegt, da Dosisempfehlungen meist aus Studien stammen, die vorwiegend mit Männern durchgeführt wurden (Chaves und Seeman 2006). Eine zunehmende Kumulation der lipophilen AP im Fettgewebe der Frauen wird als zusätzliche Ursache für stärker ausgeprägte Nebenwirkungen verstanden (Seeman MV 2009). Zudem empfinden sich Frauen als subjektiv sensibler für Antipsychotika und deren Nebenwirkungen als Männer, was zu Unterschieden bei Befragungen führen kann (Barbui et al. 2005). 


\subsection{Nikotin und Schizophrenie}

Eine Metaanalyse, die weltweit erhobene Daten auswertete, brachte hervor, dass Schizophrene gegenüber der Durchschnittsbevölkerung eine 3-fach höhere Rate an Rauchern aufweisen (de Leon und Diaz 2005). Eine Erklärung fußt auf der Vermutung, dass Stimulation zentraler Nikotinrezeptoren das kognitive Funktionsniveau verbessern kann. In den Domänen Lernen, Aufmerksamkeit und Gedächtnis konnten signifikante Verbesserungen unter Nikotinapplikation nachgewiesen werden (Levin et al. 1996; Rezvani und Levin 2001). Somit kann das Rauchen unter Schizophrenen als Selbstmedikation zur Kompensation der kognitiven Defizite angesehen werden (Hughes et al. 1986). Unter antipsychotischer Therapie steigt die Rate an Rauchern (Dalack et al. 1998). In einer Studie konnten die Haloperidol verursachten Defizite im Arbeitsgedächtnis unter zusätzlicher Applikation von Nikotin nivelliert werden (Levin et al. 1996). Nikotin scheint darüber hinaus vor einer Hochregulierung und möglichen Supersensitivität der $\mathrm{D}_{2}-\mathrm{R}$ unter AP-Therapie zu schützen (Silvestri et al. 2004). Segarra et al. konnten zeigen, dass bei Erstaufnahme rauchende Schizophrene in kognitiven Tests besser waren als nicht rauchende Schizophrene. Diese Differenz kehrte sich jedoch im Krankheitsverlauf über 12 Monate um, sodass schließlich die nichtrauchenden Patienten bessere kognitive Ergebnisse erzielten als rauchende Patienten (Segarra et al. 2010). Die Autoren deuten damit auf die Möglichkeit hin, dass Rauchen bei Ersterkrankung ein Parameter für einen schwereren Erkrankungsverlauf sein könnte. In verschiedenen Studien konnte gezeigt werden, dass Raucher wesentlich höhere antipsychotische Tagesdosen bekamen als Nichtraucher (Goff et al. 1992). Allerdings machten Tang et al. darauf aufmerksam, dass sich trotz der Dosisunterschiede die Plasmalevel von Clozapin nicht zwischen Rauchern und Nichtrauchern unterschieden (Tang et al. 2007). Auch für Olanzapin und Haloperidol konnten bei vergleichbaren applizierten APDosen bei Rauchern niedrigere Plasmaspiegel als bei Nichtrauchern beobachtet werden (Shimoda et al. 1999; Citrome et al. 2009). Dem zugrunde liegen durch Rauchen verursachte $\mathrm{CYP}_{450}$-Enzyminduktionen (z.B. CYP 450 1A2), die zu einem schnelleren Abbau und damit zu einer geringeren Wirksamkeit der verabreichten antipsychotischen Dosis führen (Zevin und Benowitz 1999).

\subsection{Ableitung der Fragestellung}

In den vorherigen Kapiteln wurden die Grundlagen der antipsychotischen Therapie, sowie deren Veränderungen über den Krankheitsverlauf und die Einwirkung auf das kognitive Funktionsniveau dargelegt. Außerdem wurden geschlechtsspezifische Unterschiede bei Schi- 
zophrenen erläutert, die auch die antipsychotische Therapie umfassen. Die wichtigsten Kernaussagen werden noch einmal aufgeführt:

1. Die Sensitivität gegenüber Antipsychotika unterscheidet sich bei Männern und Frauen (Seeman MV 2009).

2. Über den Erkrankungszeitraum kommt es zu einer Dosissteigerung antipsychotischer Therapie (Elie et al. 2010).

3. Hohe Tagesdosen von Antipsychotika sind mit kognitiven Defiziten assoziiert (Chakos et al. 2006).

Bislang liegen keine verwertbaren Angaben in der Literatur vor, die sichere Hinweise für eine kognitive Veränderung unter antipsychotischer Dosissteigerung zeigen. Die vorliegende Arbeit soll dazu beitragen, unter Berücksichtigung des Geschlechts potentielle Zusammenhänge zwischen Dosissteigerung über die Medikationsdauer und kognitivem Funktionsniveau Schizophrener zu verstehen. Es wird davon ausgegangen, dass eine Dosissteigerung nicht zu kognitiver Verbesserung führt.

Vor diesem Hintergrund sollen folgende Hypothesen geprüft werden:

1. Männer waren zum Untersuchungszeitpunkt der GRAS-Studie auf eine höhere antipsychotische Tagesdosis eingestellt als Frauen.

2. Männer erhielten zum Zeitpunkt der Erstmedikation bereits eine höhere antipsychotische Tagesdosis als Frauen.

3. Männer erfuhren über die gesamte Medikationsdauer bis zum GRASUntersuchungszeitpunkt eine größere Steigerung der antipsychotischen Tagesdosis als Frauen.

Aufgrund geschlechtsspezifischer Unterschiede zu betrachtender Testgrößen sollen die folgenden Hypothesen getrennt in der Stichprobe der Männer und der Frauen getestet werden:

4. Probanden mit hoher Dosissteigerung pro Jahr erlangen in kognitiven Leistungstests schlechtere Ergebnisse als Probanden mit moderater Dosissteigerung.

5. Probanden mit hoher Dosissteigerung pro Jahr zeigen mehr Nebenwirkungen der antipsychotischen Therapie als Probanden mit moderater Dosissteigerung.

6. Probanden mit hoher Dosissteigerung pro Jahr sind zu einem größeren Anteil auf kognitionsmodulierende Komedikation eingestellt als Probanden mit moderater Dosissteigerung.

7. Probanden mit hoher Dosissteigerung pro Jahr zeichnen sich durch höhere Krankheitsschwere aus als Probanden mit moderater Dosissteigerung. 


\section{MATERIAL UND METHODEN}

\subsection{Vorbereitungen}

Alle im Folgenden berichteten Daten der vorliegenden Arbeit wurden im Rahmen der Multicenter-Schizophrenie-Querschnittserhebung der Göttingen Research Association for Schizophrenia (GRAS) erhoben. Schwerpunkt der GRAS-Studie ist es, die in Ätiologie, Symptomatik, Verlauf und Therapierbarkeit heterogenen Erkrankungsformen der Schizophrenie durch reliable neurobiologische Kriterien in klinisch bedeutsame Subgruppen zu unterteilen und grundlegende Krankheitsprozesse zu verstehen.

Die Durchführung der GRAS-Studie wurde von der Ethik-Kommission der Georg-AugustUniversität Göttingen und den örtlichen Gremien aller teilnehmenden Zentren genehmigt. Die GRAS-Studie berücksichtigte selbstverständlich die ethischen Grundsätze der Deklaration von Helsinki (Ashcroft 2007). Um eine große und umfassende Stichprobe zu untersuchen, wurde das Design der Multicenter-Studie gewählt.

\subsection{Die Population}

Ein Untersuchungsteam des Max-Planck-Instituts für Experimentelle Medir̨in (Göttingen), welchem auch der Verfasser der vorliegenden Arbeit angehörte, reiste im Zeitraum der Jahre 2005 bis 2008 zu 23 psychiatrischen Zentren innerhalb Deutschlands (s. Anhang A), um insgesamt 1085 Probanden mit einer Diagnose aus dem schizophrenen Formenkreis zu untersuchen. Die klinisch-psychiatrisch erfahrenen Untersucher der GRAS-Studie wiesen alle einen psychologischen oder medizinischen Hintergrund auf und wurden zudem vor den Untersuchungen spezifisch geschult. Jeder Proband wurde von mindestens 2 Untersuchern begutachtet, um durch Konsensussitzungen eine größtmögliche Objektivität und Reliabilität der Untersuchungsergebnisse zu gewährleisten. Voraussetzungen, um in die Studie eingeschlossen zu werden, waren eine Diagnose aus dem schizophrenen Formenkreis oder eine schizoaffektive Störung nach DSM-IV-TR (AmericanPsychiatricAssociation 2000), sowie ein Mindestalter von 18 Jahren. Eine obere Altersgrenze war nicht festgelegt. Ebenfalls wurden Probanden aller Krankheitsstatus aufgenommen (akute, chronische, residuale, remittierte etc.). Jeder Proband erklärte nach einer ausführlichen Aufklärung über die Untersuchungen sein schriftliches Einverständnis (vgl. Anhang B). Bei etlichen Probanden war die zusätzliche Einwilligung ihrer gesetzlichen Vertreter notwenig. Die Koordinatoren der kooperierenden Zentren wählten die Studienteilnehmer nach den genannten Kriterien aus und machten diese mit dem Untersuchungsteam bekannt. 
In Tabelle 2 sind soziodemographische und klinische Charakteristika der Probanden dargestellt, die in die Analysen der vorliegenden Arbeit eingehen.

Tabelle 2: Charakterisierung der untersuchten Stichprobe anhand wesentlicher Variablen (gesamt N = 1029)

\begin{tabular}{|c|c|c|c|c|c|}
\hline & $\mathbf{n}$ & Mdn & MD & Min & Max \\
\hline Männlich & $689(67,0 \%)$ & & & & \\
\hline Weiblich & $340(33,0 \%)$ & & & & \\
\hline Alter (a) & 1029 & 39,09 & 10,31 & 17,49 & 79,49 \\
\hline Alter bei Ersterkrankung (a) & 1019 & 23,00 & 6,59 & 5,00 & 67,00 \\
\hline Erkrankungsdauer (a) & 1019 & 10,89 & 8,40 & 0,01 & 58,44 \\
\hline Dauer der unbehandelten Erkrankung (a) & 831 & 2,00 & 3,15 & 0,00 & 56,00 \\
\hline Medikationsdauer (a) & 961 & 9,00 & 7,64 & 0,00 & 49,00 \\
\hline $\begin{array}{l}\text { Aufenthaltsdauer in psychiatrischen } \\
\text { Einrichtungen (d) }\end{array}$ & 991 & 300,00 & 520,11 & 0,00 & 15674,00 \\
\hline CPÄ zum GRAS-Untersuchungszeitpunkt & 1029 & 500,00 & 437,04 & 0,00 & 7375,00 \\
\hline CPÄ zum Zeitpunkt der Erstmedikation & 841 & 300,00 & 300,74 & 0,00 & 9365,00 \\
\hline PANSS positiv & 992 & 12,00 & 4,92 & 7,00 & 38,00 \\
\hline PANSS negativ & 988 & 17,00 & 6,35 & 7,00 & 46,00 \\
\hline PANSS allgemein & 988 & 32,00 & 9,36 & 16,00 & 82,00 \\
\hline CGI & 997 & 6,00 & 0,85 & 2,00 & 8,00 \\
\hline GAF & 993 & 45,00 & 14,35 & 5,00 & 90,00 \\
\hline
\end{tabular}

CGI: Clinical Global Impression Scale, d: Tag, GAF: Global Assessment of Functioning Scale, Max: Maximum, MD: absolute mittlere Abweichung vom Median, Mdn: Median, Min: Minimum, PANSS: Positive and Negative Syndrome Scale

\subsection{Verwendete Untersuchungsinstrumente}

Innerhalb der jeweils ca. fünfstündigen Untersuchung eines jeden Probanden wurden Informationen auf der Basis strukturierter Interviews, neuropsychologischer Tests, standardisierter psychometrischer Fragebögen und Bewertungsskalen sowie einer strukturierten körperlichen und neurologischen Untersuchung erhoben. Zusätzlich wurde Blut zu genetischen und immunologischen Analysen entnommen. Zur Untersuchung auf etwaige psychiatrische Komorbidität wie z.B. Angst- und Zwangsstörungen, affektive Störungen, Substanzabhängigkeit / -abusus und zur Absicherung der Diagnose „Schizophrenie“ wurde das Strukturierte Klinische Interview für das DSM-IV Achse I: Psychische Störungen durchgeführt (Wittchen et al. 1997). Mit Hilfe weiterer strukturierter Fragebögen wurden Informationen über schulische und berufliche Entwicklung, Partnerschaften und Familienstand, Freizeitverhalten und soziales Netzwerk, juristische Vorgeschichte, häusliches Umfeld, Lebensqualität, Migrationshintergrund, Medikation, Neurotraumata, familiäre Erkrankungen, selbstverletzendes Verhalten und Suizidalität, prä- und perinatale Komplikationen, Infektionserkrankungen und somatische Erkrankungen aus der Vorgeschichte erfragt. 
Über die Untersuchungen des „Ist-Zustandes“ hinaus wurden nach schriftlichem Einverständnis der Probanden Arztbriefe früherer Klinikaufenthalte angefordert und ausgewertet. So konnten konkrete Informationen über Prodromalphase, Ersterkrankung, Erstmedikation und Krankheitsverlauf geprüft und ergänzt werden.

Im Folgenden wird ausschließlich auf die für diese Arbeit wesentlichen neuropsychologischen, psychometrischen und neurologischen Untersuchungseinheiten eingegangen. Einen vollständigen Überblick über die in der Studie verwendeten Tests und Skalen sowie entsprechende Literaturangaben bietet die Tabelle in Anhang C.

\subsubsection{Diagnose „Schizophrenie“}

Die Diagnose „Schizophrenie“ aller an der Studie teilnehmenden Probanden wurde durch das Strukturierte Klinische Interview für das DSM-IV Achse I: Psychische Störungen (Wittchen et al. 1997) verifiziert. Dieses Untersuchungsinstrument erfasst durch vorgegebene Fragen systematisch bestimmte Symptome und Syndrome nach diagnostischen Kriterien des DSM-IV-TR Achse I (AmericanPsychiatricAssociation 2000). Das DSM-IV-TR basiert die Diagnose auf 5 verschiedenen Achsen, die jeweils eine Dimension der Erkrankung oder Behinderung berücksichtigen. Die in der vorliegenden Arbeit ausschließlich genutzte Achse I erfasst das klinische Erscheinungsbild. Der Untersucher ist an Wortlaut und Reihenfolge des Fragebogens gebunden. Möglich ist jedoch, nach bestimmten Kriterien Fragen auszulassen, soweit aus dem Interview zuvor ein Nichtvorliegen des entsprechenden Syndroms hervorgeht. Vermerkt werden die Antworten des Patienten in kodierter Form. Hat der Untersucher jedoch einen von der Antwort abweichenden Eindruck, wird dieser kodiert. Durch diese Art des klinischen Interviews kann eine gute Reliabilität der Diagnosegebung gewährleistet werden (Zanarini und Frankenburg 2001).

\subsubsection{Neuropsychologische Testverfahren}

Im Folgenden werden die neuropsychologischen Testverfahren vorgestellt. Hierbei ist anzumerken, dass die Rohwerte der Testergebnisse z-transformiert wurden. Die Mediane dieser Werte stellen in den betrachteten Stichproben die primär abhängigen Variablen dar. Bis auf die computergestützte Testung der Alertness wurden alle anderen kognitiven Leistungen mittels DIN-A4 Testbogen und Stift erhoben. Alle angewandten Testverfahren sind in Anhang H1 abgebildet.

Um ein umfassendes Profil kognitiver Leistung erfassen zu können, beinhaltete die verwendete neuropsychologische Testbatterie eine große Anzahl an Inventaren und Tests, welche den Empfehlungen des MATRICS Neurocognition Committee des National Institute of Mental Health (NIMH) entsprechen (Green und Nuechterlein 2004). 
Alertness (Zimmermann und Fimm 1993)

Alertness bezeichnet den Grad zielgerichteter (phasischer) und kontinuierlicher (tonischer) Aufmerksamkeitsleistungen. In dem in der vorliegenden Arbeit verwendeten Altertness-Test werden visuelle Wahrnehmung und Aufmerksamkeit geprüft, indem die Reaktionszeit von der Darbietung eines visuellen Stimulus mit oder ohne vorherigen Warnton bis zu einer gezielten motorischen Reaktion gemessen wird. An einem Computer wird dem Probanden ein in variierenden Zeitabständen aufblinkendes weißes Kreuz vor schwarzem Hintergrund präsentiert. Die Aufgabe des Probanden ist es, so schnell wie möglich nach jedem Aufblinken des Kreuzes mit dem Zeigefinger der dominanten Hand eine spezifische Taste zu drücken. Die einfache Reaktionszeit gilt als Maß der tonischen Alertness, die Differenz zwischen den Reaktionen in den Durchgängen mit und ohne Warnton dient der Darstellung der phasischen Alertnessreaktion, d.h. der Fähigkeit der kurzzeitigen Anhebung des Aufmerksamkeitsniveaus.

Die gemittelte Reaktionszeit aller Durchgänge wird in dieser Studie als Messwert für die tonische Aufmerksamkeitsleistung verwendet.

\section{Buchstaben-Zahlen-Test (Wechsler 1998)}

Der Buchstaben-Zahlen-Test (BZT) prüft das auditorische Arbeitsgedächtnis. Defizite in dieser Domäne lassen auf Dysfunktionen des Frontalhirns schließen (Owen et al. 2005). Dem Probanden wird eine gemischte Serie von Zahlen und Buchstaben vorgelesen, die er nach einmaligem Hören in geordneter Reihenfolge wiedergeben soll: Zuerst die Zahlen in aufsteigender Reihenfolge, folgend die genannten Buchstaben in Reihenfolge des Alphabets. Der Test beginnt bei 2 Zeichen und endet bei einer Zeichenlänge von 7, wobei jeweils 4 Untertests pro Zeichenlänge durchgeführt werden. Der gesamte Test ist entweder bei Lösung des letzten Untertests oder bei fehlerhafter Wiedergabe aller 4 Beispiele eines Untertests zu Ende. Gewertet wird die Anzahl der korrekten Antworten.

\section{Dotting und Tapping (Chapman 1948)}

Diese zwei Testverfahren prüfen die visuell motorische Geschwindigkeit und Feinmotorik. Im Dotting Test wird dem Probanden ein DIN-A4-Blatt vorgelegt, auf dem 10 Reihen aus jeweils 10 ungleich weit voneinander entfernten leeren Kreisen abgebildet sind. Der Proband soll innerhalb von 30 Sekunden in vorgegebener Reihenfolge möglichst viele leere Kreise sauber zentral mit einem Punkt versehen.

Der Tapping Test ist ähnlich konzipiert, allerdings sind die einhundert leeren Kreise deutlich größer und in gleichem Abstand angeordnet. Der Proband ist ohne Vorgabe einer Reihenfolge angehalten innerhalb von 30 Sekunden in möglichst viele Kreise drei Punkte zu setzen. In beiden Tests wird die Anzahl der korrekt ausgefüllten Kreise gewertet. 


\section{Leistungsprüfsystem: Subtest 3 (Horn 1983)}

Das Leistungsprüfsystem (LPS3) erfasst schlussfolgerndes Denken, das weitgehend unabhängig von schulischer Vorbildung ist (Horn 1983). Diese „fluide Intelligenz“ ist beeinträchtigt bei globalen degenerativen Hirnprozessen (Horn 1983).

Auf dem DIN-A4-Testbogen sind 40 Reihen zu je 8 Symbolen nach logisch-formalen Gesichtspunkten angeordnet. Jeweils eines dieser 8 Symbole fällt aus dem zu erkennenden Muster. Innerhalb von 5 Minuten soll der Proband maximal viele aus der Form fallende Symbole markieren. Zur Auswertung wird die Summe der richtig bearbeiteten Einzelaufgaben bestimmt.

\section{Mehrfachwahl-Wortschatz-Intelligenztest (Lehrl 1999)}

Der Mehrfachwahl-Wortschatz-Intelligenztest Version B (MWT-B) ist ein Rekognitionstest, der wenig anfällig für psychische Störungen ist und erworbenes Wissen abfragt. Somit kann er als Schätzer des prämorbiden Leistungsniveaus (kristalline Intelligenz) genutzt werden (Lehrl 1999).

Auf dem DIN-A4-Testbogen sind 37 Wortreihen aus je fünf Wörtern aufgeführt. Je vier der fünf Wörter sind Phantasiewörter, nur eines ist richtig. Der Proband soll nun ohne Zeitlimit das ihm bekannte Wort aus jeder Zeile finden und markieren. Der Leistungswert ergibt sich aus der Summe der als korrekt erkannten Wörter. Bei einem hohen Wert an erkannten Wörtern kann ein hohes prämorbides Bildungsniveau angenommen werden, wenngleich sich die erkannten Wörter nicht im aktiven Wortschatz befinden.

\section{Trail Making Test a (Reitan 1958)}

Mit Hilfe des Trail Making Test a (TMTa) werden die Informationsverarbeitungsgeschwindigkeit und Aufmerksamkeit geprüft. Auf dem Prüfblatt sind unregelmäßig 25 Kreise verteilt, in deren Zentrum jeweils eine Zahl von 1 bis 25 gedruckt ist. Die Aufgabe des Probanden besteht darin, so schnell wie möglich die Kreise in aufsteigender Zahlenfolge mit einer gezeichneten Linie zu verbinden. Das Leistungsniveau wird in Sekunden gemessen. Je schneller der Patient ist, desto besser wird der Test bewertet.

\section{Trail Making Test b (Reitan 1958)}

Die kognitive Flexibilität und somit eine Kategorie der Exekutivfunktionen wird mit dem Trail Making Test b (TMTb) gemessen. Schlechte Ergebnisse in diesem Test werden als Korrelat einer kortikalen frontalen Dysfunktion angesehen (Stuss und Levine 2002).

Vergleichbar zum TMTa sind erneut 25 Kreise unregelmäßig auf einem Blatt verteilt. Jedoch sind die Kreise nun mit den Zahlen 1 bis 13 und den Buchstraben A bis L gekennzeichnet. 
Die Aufgabe besteht darin in Reihenfolge nacheinander abwechselnd Zahlen und Buchstaben zu verbinden (1-A-2-B-3-C usw.). Der Prüfer greift bei Fehlern korrigierend ein. Gestoppt wird die Zeit, bis das L mit der 13 verbunden ist. Je schneller der Patient ist, desto besser ist die Testbewertung.

\section{Verbaler Lern- und Merkfähigkeitstest (Helmstädter et al. 2001)}

Mit Hilfe des Verbalen Lern- und Merkfähigkeitstest (VLMT) wird das serielle verbale Lernen von Wortlisten mit nachfolgender Distraktion, Abruf nach Distraktion und halbstündiger Verzögerung, sowie das Wiedererkennen nach Distraktion geprüft. Für die vorliegende Arbeit ist lediglich die Lernleistung untersucht worden. Für die Lernleistung des deklarativen Verbalgedächtnisses wird dem Probanden 5-mal eine Liste von 15 semantisch unabhängigen Wörtern vorgelesen. Nach jedem Vorlesen der Liste wird der Proband gebeten, so viele vorgelesene Wörter wie möglich zu nennen. Gewertet wird für die Lernleistung die Summe aller korrekt genannten Wörter aus allen 5 Durchgängen.

\section{Hamburg-Wechsler Intelligenztest für Erwachsene: Zahlen-Symbol- Test (Tewes 1991)}

Der Zahlen-Symbol-Test (ZST) erfasst die allgemeine psychomotorische Geschwindigkeit und ist ein gutes Maß für Konzentration und Aufmerksamkeit (Tewes 1991).

Am oberen Ende des Testblatts ist eine Tabelle abgebildet, in der jeder Zahl von 1 bis 9 ein einfaches Symbol zugewiesen ist. Darunter sind 4 Reihen à 25 Doppelkästchen abgedruckt, wobei in jedem oberen Teil des Doppelkästchens zufällig je eine Zahl von 1 bis 9 eingetragen ist. Die Aufgabe des Probanden ist es, innerhalb von 90 Sekunden in Reihenfolge der Kästchen unter jede Zahl das entsprechende Symbol zu zeichnen. Je mehr korrekte Eintragungen die Testperson geleistet hat, desto höher ist die Punktzahl.

\subsubsection{Skalen zur Beurteilung klinischer Variablen}

In der GRAS-Studie wurden die Probanden mit Hilfe unterschiedlicher international anerkannter strukturierter Inventare und Beurteilungsskalen in klinischen Bereichen wie neurologischem Status, Krankheitsschwere, alltäglichem Funktionsniveau, Psychopathologie und Medikamentennebenwirkungen genau untersucht. Es wurde deshalb großer Wert auf diese umfassende klinische Einschätzung gelegt, um etwaige Zusammenhänge zwischen kognitiver Beeinträchtigung, psychopathologischem Erkrankungsgrad, neurologischen Ausfällen und Medikamenten/-nebenwirkungen untersuchen zu können.

Daher stellen die klinischen Variablen nach der Testung auf Stichprobendifferenzen hinsichtlich kognitiver Testergebnisse die sekundär abhängigen Variablen dar. So soll getestet werden, 
ob sich signifikante Stichprobendifferenzen klinischer Variablen ergeben, die näher im Hinblick auf die kognitiven Unterschiede zu betrachten sind.

In allen Inventaren und Skalen stellt der Median der betrachteten Stichprobe die verwendete Berechnungsgröße dar. Alle verwendeten Skalen zur Beurteilung klinischer Variablen sind im Anhang $\mathrm{H} 2$ bis $\mathrm{H} 5$ zusammengefasst.

\subsubsection{Neurologische Beurteilungsskala}

\section{Cambridge Neurological Inventory (Chen et al. 1995)}

Mit Hilfe des strukturierten neurologischen Einschätzungsinstrumentes psychiatrischer Patienten Cambridge Neurological Inventory (CNI) werden insgesamt 114 Untersuchungskriterien auf einer Skala von 0 (normal) bis 2 (extrem auffällig) bei jedem Probanden beurteilt. Betrachtet werden neurologische „soft-signs“, organisch-neurologische Ausfälle („hardsigns") und EPMS. Neurologische Abweichungen auf motorischer, sensorischer, oder integrativer Ebene sind als Neurologische „soft-signs“ charakterisiert. Im Gegensatz zu den „hardsigns“ können ihnen generalisierte, jedoch keine klar lokalisierten und definierten Läsionen im zentralen Nervensystem (ZNS) zugeordnet werden (Quitkin et al. 1976). Schizophrene zeigen als Korrelat eines evtl. degenerativen Hirnprozesses und reduzierter neuronaler Integrität eine wesentlich höhere Inzidenz dieser „soft-signs“ als Gesunde (Rossi et al. 1990). Es konnte eine Assoziation von ,soft-signs“, Krankheitsschwere und kognitiver Beeinträchtigung nachgewiesen werden (Liddle 1987).

Addiert man alle 114 Items des CNI zusammen, ergibt sich ein umfassender Summenwert neurologischer Beeinträchtigung. Andererseits können die Beobachtungen zu 8 Subskalen addiert werden: 1. organisch-neurologische Defizite, 2. motorische Koordination, 3. Sinneswahrnehmung/-verarbeitung, 4. Primitivreflexe, 5. tardive Dyskinesien, 6. Zeichen der Katatonie, 7. Parkinsonismus und 8. Einschränkung der Reaktionsunterdrückung.

In der vorliegenden Arbeit wird der durch die Summe der untersuchten Items geteilte Summenwert betrachtet, um eine Aussage über den globalen neurologischen Gesundheitszustand treffen zu können.

\subsubsection{Skala zur Beurteilung der Krankheitsschwere \\ Clinical Global Impression Scale (Guy 1976)}

Mit der international anerkannten Clinical Global Impression Scale (CGI) können drei GröBen bei psychisch Erkrankten abgefragt werden: 1. Schweregrad der Krankheit, 2. Heilungsverlauf und 3. Wirksamkeit der Therapie. In der vorliegenden Arbeit wird allein auf die erste Größe „Krankheit“ eingegangen, da im Rahmen der Querschnittsuntersuchung keine Verlaufsbeobachtungen geleistet werden konnten. Die CGI gibt eine 7-stufige Ordinalskala von 2 
„Patient ist überhaupt nicht krank“ bis 8 „Patient gehört zu den extrem schwer Kranken“ vor, auf der der Proband eingeschätzt wird. Unter dem Punkt 1 wird „nicht beurteilbar“ vermerkt.

\subsubsection{Skala zur Beurteilung des Funktionsniveaus \\ Global Assessment of Functioning Scale (Endicott et al. 1976)}

Die international gebräuchliche Global Assessment of Functioning Scale (GAF) hilft bei der Beurteilung psychischer, sozialer und beruflicher Leistungsfähigkeit auf einer theoretischen Spanne zwischen seelischer Gesundheit und Krankheit, ohne dass körperliche oder umweltbedingte Einschränkungen einfließen. Die ordinale Skala von 1 (geringe Leistungsfähigkeit) bis 100 (maximale Leistungsfähigkeit) gibt in Zehnerabständen exemplarisch psychische, soziale und berufliche Einschränkungen in zunehmender Intensität an. Anhand dieser wird der Zustand des Probanden bei der Untersuchung auch in Zwischenwerten eingeschätzt.

\subsubsection{Skala zur Beurteilung der Psychopathologie}

\section{Positive and Negative Syndrome Scale (Kay et al. 1987)}

Zur Erfassung des Schweregrades eines breiten Spektrums psychopathologischer Symptome Erwachsener ist die Positive and Negative Syndrome Scale (PANSS) ein international etabliertes und standardisiertes Fremdbeurteilungsinstrument. 30 verschiedene Symptome werden nach strikter Definition jeweils nach Schweregrad auf einer Skala von 1 (nicht vorhanden) bis 7 (extrem) erhoben. Jeweils sieben Symptome können zur Subskala „Positivsymptome“ bzw. zur Subskala „Negativsymptome“ subsumiert werden. Die Summe der übrigen Items ergibt die „Allgemeine Psychopathologie“ (vgl. Seite 2 f.). Die „Allgemeine Psychopathologie“ berücksichtigt Beobachtungen zu z.B. Angst, Depression, körperlichen Symptomen und dem Kontakt zwischen Untersucher und Proband.

Nach Muller und Wetzel sind reliable Untersuchungsergebnisse erst nach drei Trainingssitzungen gewährleistet (Muller und Wetzel 1998). In der GRAS-Studie wurde deshalb dieses Instrument erst nach Übungssitzungen und Schulungen von den Untersuchern angewandt. Um zusätzliche Validität und Reliabilität zu gewährleisten, wurde jeder Proband von mindesten 2 Untersuchern in Konsenssitzungen auf Grundlage der PANSS beurteilt.

\subsubsection{Skalen zur Einschätzung der Medikamentennebenwirkungen}

Auf Seite $8 \mathrm{f}$. wurde kurz auf Nebenwirkungen der Antipsychotika eingegangen. Da die Nebenwirkungen sowohl einen entscheidenden Effekt auf den Behandlungsverlauf, als auch die Interpretation motorisch-neuropsychologischer Tests ausüben, wurden sie anhand verschiedener operationalisierter Skalen bewertet.

Zuvor sollen für das Verständnis noch wichtige Begrifflichkeiten erläutert werden: 
Dyskinesien sind verschiedene Formen ungewollter Bewegungsstörungen wie Tics im Gesichtsbereich, choreiforme (spontane, schnelle, unregelmäßige) oder athoide (langsame, unregelmäßige, komplexe, schlangenartige) Bewegungen der Extremitäten oder des gesamten Rumpfes. Auch können Muskelverkrampfungen auftreten, die als Dystonien dem Überbegriff Dyskinesien zugeordnet sind. Durch antipsychotische Medikation hervorgerufen treten Frühdyskinesien meist innerhalb der ersten Medikationswoche in Abhängigkeit zur Geschwindigkeit der Dosissteigerung auf und äußern sich in Zungen-, Schlund- und Blickkrämpfen. Sie können mit Anticholinergika erfolgreich unterdrückt werden. Spätdyskinesien hingegen treten als irreversible und nicht behandelbare Komplikation nach antipsychotischer Langzeitbehandlung auf.

Akathisie bezeichnet eine extrapyramidale Hyperkinesie, die durch eine massive innere Unruhe des Betroffenen bedingt ist. Durch kurze periodische Bewegungen versuchen die Betroffenen die leidvolle Anspannung abzubauen. Die Beeinträchtigung kann so massiv sein, dass Sitzen, Liegen und Schlafen unmöglich werden. Ungefähr eine Woche nach Therapiebeginn mit Antipsychotika kann es zu der akuten und reversiblen Form der Akathisie kommen, die mit Anticholinergika, Betablockern oder Benzodiazepinen teilweise unterdrückt werden kann. Nach langjähriger antipsychotischer Therapie kann es zu tardiven oder Spätdyskinesien kommen, die bislang nicht medikamentös behandelbar sind (Bratti et al. 2007). Nachfolgend werden die angewandten Skalen vorgestellt, welche akute und tardive Dyskinesien, Akathisien und Dystonien erfassen.

\section{Abnormal Involuntary Movement Scale (Guy 1976)}

Die insgesamt 10 Items der Abnormal Involuntary Movement Scale (AIMS) fragen auf einer ordinalen Skala von 0 (kein/minimal) bis 4 (schwer) nach Symptomen der Dyskinesie, wobei die ersten sieben Items zum Summenscore der Bewegungen von Gesicht, Extremitäten und Körperstamm addiert werden. Items 8 und 9 erfassen den Schweregrad und die Behinderung der abnormalen Bewegungen. Item 10 erfragt den subjektiven Bewußtseinsgrad des Probanden über die Bewegungen. Zwei dichotome weitere Items erfragen das Vorhandensein oder das grundsätzliche Tragen einen Gebisses.

In der vorliegenden Arbeit wird der Summenwert aus Item 1 bis 7 verwendet. 
Barnes-Akathisia Scale (Barnes 1989)

In den Kategorien „Objektiv: Vorhandensein“, „Subjektiv: Bemerken“, „Subjektiv: Belastung“ und „Globale Klinische Akathisie Beurteilung“ wird mit der Barnes-Akathisia Scale (BAS) die Akathisie bewertet. In den ersten drei Kategorien können Werte von 0 (nicht vorhanden) bis 3 (maximal) erreicht werden, die letzte Kategorie ist in fünf Werte unterteilt. In der vorliegenden Arbeit wird die Kategorie „Globale klinische Akathisie Beurteilung“ betrachtet.

\section{Simpson-Angus Scale (Simpson und Angus 1970)}

In den ersten 7 Items der Simpson-Angus-Scale (SAS) wird dystonische Rigidität (Muskelsteife) klassifiziert, in Item 8 die Ermüdbarkeit des Glabella-Tipp-Reflexes, in Item 9 Tremor und in Item 10 Speichelfluss. Jedes Item kann die Werte 0 (normal) bis 4 (extrem) annehmen. Für die Untersuchungen in dieser Arbeit wurde der Summenwert aller Items angewandt.

\section{Tardive Dyskinesia Rating Scale (Simpson et al. 1979)}

Mit Hilfe der Tardive Dyskinesia Rating Scale (TDRS) wird die tardive Dyskinesie durch 34 fest vorgegebene Items der Kategorien Gesicht, Nacken/Rumpf, obere Extremitäten, untere Extremitäten sowie Körperhaltung erhoben. Der Untersucher kann zwei Zusatzbewertungen pro Kategorie ergänzen, sodass insgesamt 44 Items zu einem Summenwert addiert werden. Ein jedes Item ist von 0 (nicht vorhanden) bis 5 (sehr schwer) zu bewerten. In die Analysen der vorliegenden Arbeit geht der Summenwert der TDRS ein.

\subsubsection{Angegebene Nebenwirkungen}

Innerhalb der strukturierten Interviews wurden den Probanden offene Fragen zu Medikamentennebenwirkungen gestellt, um eine subjektive Einschätzung zu Nebenwirkungen seitens des Probanden zu erheben. Die geäußerten Nebenwirkungen wurden in die vier Kategorien kardiovaskuläre, metabolisch-endokrine, neurologische und psychiatrische Nebenwirkungen eingeteilt und zu dem Summenwert aller Nebenwirkungen zusammengefasst. Eine Übersicht der den 4 Kategorien zugeordneten Nebenwirkungen ist dem Anhang D zu entnehmen.

\subsection{Berechnete Variablen}

\subsubsection{Antipsychotische Medikation und das Chlorpromazinäquivalent}

Da die antipsychotische Medikation in Wirkstoff, Dosis und Applikationsart zwischen den Probanden differierte, bestand ein Teil der Arbeit darin, die Medikation der Probanden in eine vergleichbare Einheit - das Chlorpromazinäquivalent (CPÄ) - umzuwandeln. Delay und Deni- 
cker stellten 1952 Chlorpromazin als erstes antipsychotisches Medikament vor, welches seither mit einer neuroleptischen (antipsychotischen) Potenz von 1 pro $1 \mathrm{mg}$ als Referenzdosis gilt (Haase und Janssen 1965).

Der Vergleichbarkeit der antipsychotischen Wirkstärke später entwickelter AP liegt ein Gedanke von H.J. Haase (1965) zugrunde. Er nutzte die Doppelwirkung der antipsychotisch wirksamen Dosis auf kortikale und striatale $\mathrm{D}_{2}-\mathrm{R}$ (vgl. Seite 8 f.), indem er unter Aufdosierung der AP aufgetretene EPMS mittels Handschrifttest quantifizierte. Die minimale Dosis eines Wirkstoffs, die zu noch gerade detektierbaren EPMS führte, nannte er „,neuroleptische Schwellendosis“. Diese Schwellendosen der damals vorhandenen Antipsychotika setzte er in Vergleich zu der des Chlorpromazins und bildete so den ChlorpromazinUmrechnungskoeffizient (CPK) eines jeden AP. Je höher die Affinität eines Wirkstoffs zu dem $\mathrm{D}_{2}-\mathrm{R}$, desto schneller ergeben sich EPMS pro Dosis, desto höher ist dessen CPK und folglich auch die antipsychotische Potenz (Haase und Janssen 1965).

Da sich aber sowohl inter- als auch intraindividuelle Abweichungen der „,neuroleptischen Schwellendosis“ ergeben können, ist der CPK lediglich ein Schätzer (Haase und Janssen 1965; Rey et al. 1989). Für die SGA ist aufgrund des Wirkungsspektrums auf verschiedene zentrale Rezeptoren der CPK über die „,neuroleptische Schwellendosis“ nicht bestimmbar. Deshalb sind in der Vergangenheit für die SGA CPK durch unterschiedliche Ansätze bestimmt worden (z.B. klinische Vergleichsstudien, Vergleiche der durchschnittlichen Tagesdosen, in Relation gesetzte Blutplasmakonzentrationen, Erfahrungswerte und Einschätzungen aus Tiermodellen; vgl. Anhang E und F). Nach ausführlicher Literaturrecherche konnte für alle 47 oral und 12 in Depotform applizierten Wirkstoffe, auf die die Probanden unserer Studie eingestellt waren, ein passender Umrechnungsfaktor gefunden werden. Diese sind dem Anhang E und F zu entnehmen.

Bei jedem Patienten wurden alle zum GRAS-Untersuchungszeitpunkt oral und in Depotform verabreichten antipsychotischen Wirkstoffe in entsprechender Dosis in CPÄ umgerechnet und zur antipsychotischen Gesamttagesdosis addiert. Genauso wurde bei der Errechnung der Erstmedikation verfahren. Als Erstmedikation wurde die erste bekannte antipsychotische Medikation angegeben, die für mindestens einen Monat kontinuierlich verabreicht worden war. Diese Regel wurde befolgt, um die antipsychotische Basismedikation und nicht etwaige temporäre Extremdosen als Erstmedikation zugrunde zu legen.

\subsubsection{Das Delta-Chlorpromazinäquivalent}

In der vorliegenden Arbeit stellt die antipsychotische Dosissteigerung über die Zeit die zu untersuchende Einflussgröße auf die Kognition dar und ist somit die unabhängige Variable. Für die Auswertungen waren 2 antipsychotische Dosen bekannt: die erste zum Zeitpunkt der 
Erstmedikation, die zweite zum Zeitpunkt der GRAS-Untersuchung. Nach Errechnung der gesamten Chlorpromazintagesdosen zu den 2 Zeitpunkten wurde die Differenz der beiden Dosen durch Subtraktion bestimmt. Dieser Wert wurde anschließend durch die Anzahl der Medikationsjahre dividiert, um den Zeitfaktor berücksichtigen zu können. Das DeltaChlorpromazinäquivalent $(\Delta \mathrm{CP} \ddot{A})$ hat die Einheit $\mathrm{mg} / \mathrm{J}$ und stellt einen groben Schätzwert des Dosisverlaufes dar, weil die Errechnung aus Werten von 2 Zeitpunkten erfolgte und somit nicht dem Anspruch einer wahren longitudinalen Beobachtung genügt.

\subsubsection{Gesamtdauer psychiatrischer Aufenthalte}

Hoffman und McGlashan (1993) legten nahe, dass lang andauernde psychotische Phasen einen folgeschweren, pathologischen Effekt auf das ZNS haben und zu schlechten Langzeitergebnissen führen können (Hoffman und McGlashan 1993). Auch wurde vermutet, dass in der Anfangsphase einer jeden Rückfallepisode ein weiterer Degenerationsschub kognitiver Leistungen stattfindet (Breier et al. 1991). Da in $\operatorname{der}$ GRAS-Querschnittsuntersuchung nicht die Summe der Tage mit psychotischer Symptomatik erfasst werden konnte, wurde durch Aufarbeitung der eingeforderten Arztbriefe die Gesamtdauer psychiatrischer Krankenhausaufenthalte zusammenaddiert. Dieser kumulative Wert wird in der vorliegenden Arbeit als Schätzer der Krankheitsschwere angesehen (Johnstone et al. 1981; Harvey et al. 2010).

\subsubsection{Dauer der unbehandelten Erkrankung}

Die Dauer der unbehandelten Erkrankung oder Psychose (DUP) wurde aus zwei Variablen berechnet. Zum einen wurde aus dem Probandeninterview oder den Arztbriefen der Störungsbeginn erhoben. Erfasst wurden darin Symptome des Prodroms wie z.B. Unruhe, Depressionen, Angstzustände, mangelndes Selbstvertrauen, sozialer Rückzug oder der (schulische) „Leistungsknick“. Zum anderen wurde aus den Arztbriefen der Zeitpunkt der ersten Medikation erhoben. Die Differenz dieser beiden Zeitpunkte ging als DUP in die Analysen ein (Rabiner et al. 1986).

\subsection{Aufteilung der Stichprobe}

Um eine statistische Vergleichbarkeit in den neuropsychologischen Tests voraussetzen zu können, wurden in der vorliegenden Arbeit nur eindeutig schizophrene Patienten berücksichtigt, die entweder mit deutsch als Muttersprache, oder deutsch-bilingual aufgewachsen waren. Aus den auf Seite 12 f. dargelegten Gründen wurde zur statistischen Prüfung aller Hypothesen die Gesamtstichprobe in die Teilstichproben Männer und Frauen geteilt. Hypothese 1 konnte an 1029 Probanden (689 Männer und 340 Frauen) getestet werden, von denen die komplette 
Medikation zum GRAS-Untersuchungszeitpunkt in CPÄ umgerechnet werden konnte. Die Medikation zum Zeitpunkt der Ersterkrankung war von 841 Probanden hinreichend bekannt. Somit konnte die Hypothese 2 an 565 Männern und 276 Frauen getestet werden. Die Hypothese 3 konnte an 753 Probanden getestet werden, die sich aus 512 Männern und 241 Frauen zusammensetzten.

Nachfolgend wurden auch die Hypothesen 4 bis 7 parallel in der Männer- und Frauenstichprobe getestet. In beiden Gruppen wurde für das $\Delta \mathrm{CPÄ/J}$ zusätzlich ein Mediansplit durchgeführt. Dieser teilte die Frauen- und Männerstichprobe nach der Höhe des $\Delta \mathrm{CPÄ/J}$ in zwei gleich große Subgruppen ein. Diejenigen Probanden mit höherem $\triangle \mathrm{CP} \ddot{A} / \mathrm{J}$ als dem $\triangle \mathrm{CP} \ddot{A}$ Medianwert $(\triangle \mathrm{CPÄ} / \mathrm{J} \uparrow)$ wurden denjenigen mit geringerem $\Delta \mathrm{CP} \ddot{A}$ als dem $\Delta \mathrm{CP} \ddot{\mathrm{A}}$ Medianwert $(\triangle \mathrm{CPÄ} / \mathrm{J} \downarrow)$ gegenübergestellt. Probanden, die genau den $\Delta \mathrm{CP} \ddot{\mathrm{A}} / \mathrm{J}$-Wert des Medians besaßen, wurden mittels zufälliger Zuordnung durch die Bernoulli-Verteilung mit $\mathrm{p}=0,5$ den jeweiligen 2 Subgruppen zugeteilt. Bei einem $\Delta \mathrm{CPÄ/J-Medianwert} \mathrm{von} \mathrm{17,39mg/J} \mathrm{stan-}$ den in der Stichprobe der Männer 256 Probanden mit hohem $\Delta$ CPÄ/J 256 Probanden mit niedrigem $\Delta \mathrm{CPÄ/J}$ gegenüber. Bei den Frauen lag der $\Delta \mathrm{CPÄ/J-Medianwert} \mathrm{bei} \mathrm{7,85mg/J} \mathrm{und}$ teilte die Stichprobe in 121 Frauen mit hohem und 120 Frauen mit niedrigem $\Delta$ CP ̈̈/J.

\subsection{Statistische Verfahren}

Alle durchgeführten Berechnungen basieren auf dem Stand der Datenbank vom 25.05.2010. Die Auswertungen wurden mit der Software Statistical Package for the Social Sciences (SPSS), Version 17.0 durchgeführt. Zu Anfang der Auswertungen wurden alle primär und sekundär abhängigen Variablen, die in die Berechnungen eingehen, mittels Kolmogorov-Smirnov-Test (K-S-T) auf Normalverteilung geprüft (Field 2009). Da keine der betrachteten Variablen der Normalverteilung folgte und/oder diese zusätzlich nicht intervall- oder verhältnisskaliert vorlagen, waren die Kriterien der parametrischen Testung nicht erfüllt (Bortz 1999). Deshalb wurden nichtparametrische Verfahren angewandt. In der vorliegenden Arbeit wird somit als Lageparameter der Median (Mdn) angegeben und als Streumaß die mittlere absolute Abweichung vom Median (MD). Die Festlegung des Signifikanzniveaus erfolgte für alle durchgeführten Tests auf einen Wert von $\mathrm{p}=0,05$.

Die Paarkontraste der Hypothesen 1 bis 4 wurden mittels des non-parametrischen U-Testes nach Mann-Whitney berechnet (Bortz 1999). Dieser Test wird als verteilungsfreies Analogon des t-Tests verstanden und vergleicht zwei unabhängige Stichproben hinsichtlich der zentralen Tendenz. Um eine etwaige konsistente Assoziation bekannter Variablen (vgl. Einflussgrößen der Kognition) mit kognitiven Einzeltests zu quantifizieren, wurden im Vorfeld der vorliegenden Arbeit Regressionsanalysen durchgeführt. Allein das Alter wurde als Einflussgröße auf 
alle kognitiven Tests identifiziert und hinsichtlich der Prüfung der Hypothese 4 mittels einfaktorieller Kovarianzanalysen (ANCOVA) als Kovariate in die Analysen einbezogen. Mit dem Chi-Quadrat-Test wurden Zusammenhänge von Gruppenzugehörigkeit und klinischer Variablen zwischen den Geschlechtern sowie den jeweils $2 \Delta$ CPÄ/J-Gruppen geprüft. K-S-T wurden zur Testung der Gruppenunterschiede in den psychopathologischen Beurteilungsvariablen sowie der gesamten subjektiven Medikamentennebenwirkungen angewandt. Zur Testung der Gruppenunterschiede im Hinblick auf die Zusatzmedikation und die 4 Kategorien subjektiver Nebenwirkungen kamen Binomial-Tests zur Anwendung.

Die Hypothesen 4 bis 7 wurden multipel getestet. D.h., dass für die Annahme der jeweiligen Hypothese unterschiedliche Variablen mit dem gleichen Signifikanztest geprüft wurden. Diese Tatsache macht die Korrektur des Signifikanzniveaus notwendig. In der vorliegenden Arbeit wurde deshalb die konservative Bonferroni-Korrektur angewandt (Bortz 1999). Bei der Darstellung der Ergebnisse sind die Signifikanzen der Einzeltests dargestellt. Signifikanzen unter Berücksichtigung der Bonferroni-Korrektur sind in den Grafiken und Tabellen zusätzlich mit blauen Sternchen gekennzeichnet.

\section{Aufbereitung der Variablen}

Die z-transformierten Rohwerte der neuropsychologischen Testergebnisse stellten die Grundlage der statistischen Analysen dar. Die z-Transformation führt zu einer besseren Vergleichbarkeit von Prüfwerten verschiedener Tests, weil die individuellen Leistungen eines jeden Tests an der Gesamtleistung der Stichprobe relativiert werden. Jeder Rohwert wird durch die Subtraktion des Mittelwertes und die anschließende Division der Standardabweichung der Gesamtverteilung aller Testwerte standardisiert. Dadurch führt die z-Transformation zu einer Verteilung mit dem Mittelwert von 0 und eine Streuung von 1. Die Lage des Wertes innerhalb der Gesamtstichprobe wird nicht verändert (Bortz 1999).

Alle abhängigen Variablen der kognitiven Tests wurden so gepolt, dass eine größere Leistung einen höheren Punktwert ergab. Das bedeutete eine Umkehrung des Vorzeichens bei den Tests, bei denen die Arbeitsgeschwindigkeit durch die Zeit gemessen wurde (Alertness, TMTa, TMTb).

Mit der Reliabilitätsanalyse wurde getestet, welche neuropsychologischen Testsergebnisse zu einem neuropsychologischen Summenwert zusammenaddiert werden konnten. Die Reliabilitätsanalyse prüft anhand verschiedener Kriterien, welche einzelnen Tests sich für einen Gesamttest als brauchbar oder unbrauchbar herausstellen (Lienert und Raatz 1998). Die in dieser Analyse beschreibende Größe „Cronbachs alpha“ kann einen Wert zwischen - $\infty$ und 1 annehmen, wobei ab einem Wert >0,70 eine gute innere Konsistenz angenommen werden kann. Das Cronbach alpha zeigte mit einem Wert von 0,879 eine gute Reliabilität der gemeinsamen 
Testung der Kognition durch die Tests Alertness, BZT, Dotting, LPS3, MWTB, Tapping, TMTa, TMTb, VLMT und ZST. Diese wurden zum kognitiven Summenwert zusammenaddiert.

\section{Testung der Paarkontraste}

Wie auf Seite 30 f. erwähnt, besteht die vorliegende Arbeit aus Untersuchungen unterschiedlicher Stichproben. So wird die Unterschiedshypothese hinsichtlich der antipsychotischen Medikation und antipsychotischen Dosissteigerung über die Medikationsdauer zwischen Männern und Frauen getestet. Außerdem werden in paralleler Betrachtung der Geschlechter Unterschiedshypothesen hinsichtlich der Kognition und klinischer Parameter zwischen Individuen hoher antipsychotischer Dosissteigerung $(\Delta \mathrm{CPÄ} / \mathrm{J} \uparrow)$ und denen geringerer antipsychotischer Dosissteigerung $(\triangle \mathrm{CP} \ddot{\mathrm{A}} / \mathrm{J} \downarrow)$ getestet.

Der U-Test nach Mann und Whitney wurde zur Testung der antipsychotischen Gruppenunterschiede zwischen Männern und Frauen, sowie zur Testung der kognitiven Gruppenunterschiede zwischen den jeweils zwei $\Delta \mathrm{CPÄ/J-Gruppen} \mathrm{beider} \mathrm{Geschlechter} \mathrm{genutzt.} \mathrm{Ebenfalls}$ wurden in den Stichproben die Variablen Alter, Alter bei Ersterkrankung, Erkrankungsdauer, DUP, Gesamtdauer psychiatrischer Aufenthalte und Medikationsdauer durch den U-Test geprüft. Es ist der gebräuchlichste nichtparametrische Test zum Vergleich zweier unabhängiger Stichproben, der auf dem Vergleich einer gemeinsamen Rangreihe der Werte beider Stichproben basiert. Dem jeweils kleinsten Wert wird der Rangplatz 1 zugeteilt. Der Test vergleicht die Stichproben hinsichtlich ihrer zentralen Tendenz (Median).

Der Kolmogorov-Smirnov-Test (K-S-T) fand im Hinblick auf unterschiedliche Aspekte der Datenauswertung Verwendung. Zum einen wurde der Test genutzt, um zu prüfen, ob zu untersuchende Variablen in der Grundgesamtheit normalverteilt waren (s.o.). Zum anderen wurden die Gruppenunterschiede der Variablen klinischer Skalen (PANSS, CGI und GAF) sowie der Gruppenvergleich der Summe aller subjektiv genannten Medikamentennebenwirkungen durch dieses Testverfahren geprüft. Der K-S-T ist als nichtparametrischer Test für rangskalierte und zusätzlich nicht der Normalverteilung folgenden Variablen geeignet (Bühl 2006).

Die Gruppenunterschiede zwischen den jeweils zwei $\Delta \mathrm{CPÄ/J-Gruppen} \mathrm{hinsichtlich} \mathrm{der} \mathrm{sub-}$ jektiv angegebenen Medikamentennebenwirkungen wurden mit dem Binomialtest geprüft. Dieser Test wurde angewandt, da die Merkmale der 4 Hauptkategorien dichotom vorlagen, d.h. nur die Ausprägung ,ja“ oder „,nein“ annehmen konnten.

Gruppenunterschiede im Patientenstatus, in der Applikationsart der AP, im Typus der applizierten AP, in der Summe der applizierten AP, im Raucherstatus und im Verhältnis von Probanden mit CPÄ Dosis $>1000 \mathrm{mg} / \mathrm{d}$ zu $<1000 \mathrm{mg} / \mathrm{d}$ zwischen den Geschlechtern, sowie der

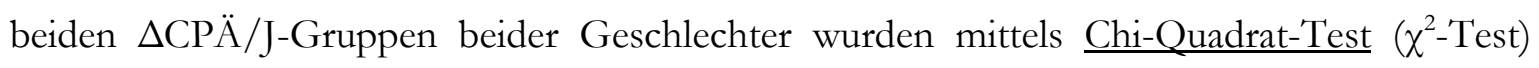


geprüft. Der Chi-Quadrat-Test wurde als Unabhängigkeitstest genutzt, um zu testen, ob zwei Merkmale, die beliebig skaliert sein können, statistisch unabhängig voneinander vorliegen (Bortz 1999). Auch kann er für die Testung stochastischer Unabhängigkeit zweier dichotomer Variablen genutzt werden.

\section{Einflussgrößen der Kognition}

Es ist bekannt, dass Alter, prämorbides Leistungsniveau, psychopathologisches Erscheinungsbild, Medikation (Dauer, Dosis, Typus), Negativsymptomatik, Medikamentennebenwirkungen und Komedikation Einflussgrößen kognitiver Testungen sind (Harvey et al. 2004; Hori et al. 2006). Für die in der vorliegenden Arbeit beschriebene Stichprobe wurden im Vorfeld dieser Arbeit systematische Regressionsanalysen durchgeführt. Somit sollten stetige Beziehungen zwischen den einzelnen abhängigen Variablen (kognitive Testergebnisse) und der o.g. unabhängigen Variablen festgestellt und quantifiziert werden. Die Variable $\Delta \mathrm{CP} \ddot{A} / \mathrm{J}$ Gruppe wurde fest als Variable eingeschlossen. Folgende Variablen wurden im Rückwärtsverfahren betrachtet: PANSS-positiv-Skala, PANSS-negativ-Skala, PANSS-allgemein-Skala, Benzodiazepineinnahme, Einnahme von Anticholinergika, CGI, GAF, DUP, Erkrankungsdauer, Alter, MWTB, SAS.

Ausschließlich die Variable Alter konnte unter Berücksichtigung des Faktors $\Delta \mathrm{CP} \ddot{\mathrm{A}} / \mathrm{J}$-Gruppe als konsistente Einflussgröße auf die neuropsychologischen Testswerte identifiziert werden. Das Alter korrelierte negativ mit kognitiver Leistung aller durchgeführten Tests. Aus diesem Grund floss nur das Alter als Kovariate in die Kovarianzanalysen (ANCOVAs) ein. Dieses Ergebnis ist übereinstimmend mit Analysen aus der MATRICS Consensus Cognitive Battery, die als wesentlichste Störgrößen kognitiver Testung die Variablen Geschlecht und Alter herausstellen (Kern et al. 2008).

\section{Testung der postulierten Gruppenunterschiede}

Um den Alterseffekt bei der Testung von Hypothese 4 zu berücksichtigen, wurde eine einfaktorielle Kovarianzanalyse (ANCOVA) durchgeführt. Dieses statistische Verfahren dient der Überprüfung der Frage, inwieweit eine abhängige Variable von einer unabhängigen (metrischen) Variablen und/oder einem Faktor beeinflusst wird (Bühl 2006).

Für jeden kognitiven Testwert wurde in den verschiedenen Stichproben eine ANCOVA in 3 Schritten durchgeführt. Im ersten Schritt wurde die Assoziation vom Faktor $\Delta C P A ̈ / J-G r u p p e$ mit kognitiven Einzeltests geprüft. Im zweiten Schritt wurde das Modell um die Kovariate „Alter“ ergänzt. Unter Einschluss des Alters wurde im dritten Schritt geprüft, ob der Faktor $\Delta \mathrm{CP} \ddot{A} / \mathrm{J}$-Gruppe im gesamten Modell noch signifikanten Einfluss auf die Gruppenunterschiede hinsichtlich der kognitiven Einzeltests zeigte, oder ob die Unterschiede allein durch 
das Alter verursacht waren. Diese Ergebnisse sind in der vorliegenden Arbeit mit „Faktor innerhalb des Modells" bezeichnet.

Die ANCOVA setzt eine Normalverteilung für die abhängigen Variablen voraus. Deshalb wurden für die Analysen diese Variablen mit der Blomtransformation standardisiert, sodass eine Normalverteilung mit einem Mittelwert von 0 und einer Varianz von 1 erreicht wurde (Bühl 2006). 


\section{ERGEBNISSE}

\subsection{Verteilung der antipsychotischen Dosis zum GRAS- Untersuchungszeitpunkt}

Zu Anfang der Untersuchungen stellte sich die Frage, ob sich Männer und Frauen bezüglich der antipsychotischen Tagesdosis unterscheiden. Um die Quantität der Medikation aller Probanden vergleichen zu können, wurde die gesamte antipsychotische Medikation eines jeden Patienten in Chlorpromazinäquivalente (CPÄ) umgerechnet. Das konkrete Vorgehen ist auf Seite $28 \mathrm{f}$. beschrieben.

Die Abbildungen 1 bis 3 sowie Tabelle 3 stellen die deskriptiven und inferenzstatistischen Kennwerte der durchschnittlichen antipsychotischen Tagesdosen innerhalb der Gesamt-, Männer- und Frauenstichprobe im Überblick dar. Es ist ersichtlich, dass sich die Medianwerte der Gesamt- (500mg $\pm 437 \mathrm{mg})$, der Männer- (520mg $\pm 440 \mathrm{mg})$ und der Frauenstichprobe (450mg $\pm 428 \mathrm{mg})$ in Bezug auf die CPÄ-Dosisverteilung deutlich unterscheiden. Weiterhin zeigen die Grafiken, dass die Werte in allen Stichproben rechtsschief verteilt sind und deutlich von der eingezeichneten Normalverteilungskurve abweichen. Die Stichprobe der Männer zeigt im Vergleich zu der Frauenstichprobe eine größere Ausprägung im Mittelwert, im Median sowie der mittleren absoluten Abweichung vom Median und kleinere Werte in der Standardabweichung, Varianz, Rechtsschiefe, Kurtosis und dem Maximum. Das bedeutet, dass Männer eine größere mittlere antipsychotische Tagesdosis zum GRASUntersuchungszeitpunkt bekamen als Frauen. Zudem zeigen die Werte der Standardabweichung und Varianz, dass die einzelnen antipsychotischen Tagesdosen bei Frauen zu einem höheren Maß um den Mittelwert der antipsychotischen Tagesdosis streuen, als bei Männern. Jedoch zeigen die Werte der mittleren absoluten Abweichung vom Median, dass die Streuung um den Median in der Stichprobe der Männer größer ist als in der Frauenstichprobe. Die Rechtsschiefe ist in allen betrachteten Stichproben damit zu erklären, dass die Werte rechtsseitig des Mittelwertes in einem größeren Maß sehr hohe Werte annehmen können als linksseitig, da die antipsychotische Medikation nur positive Werte annehmen kann. Die höheren Werte der Streuung um den Mittelwert und auch der Maximaltagesdosis antipsychotischer Medikation bei Frauen im Vergleich zu Männern erklären den breiteren Gipfel der Verteilung (Kurtosis) in der Stichprobe der Frauen.

Da sich die Gesamtstichprobe aus den Stichproben von Frauen und Männern zusammensetzt, zeigen die statistischen Kennwerte der Männer im Vergleich zu denen der Gesamtstichprobe die gleiche Tendenz, wie im Vergleich zu denen der Frauen. Allerdings sind die Differenzen 
zwischen den Werten der Stichproben kleiner. Das Gleiche gilt für den Vergleich der statistischen Kennwerte der Frauen im Vergleich zu denen der Gesamtstichprobe.

Der U-Test zeigte, dass hinsichtlich der antipsychotischen Tagesdosis vorhersagekonform mit Hypothese 1 ein signifikanter Unterschied zwischen Männern und Frauen besteht $(z=2,361$, $\mathrm{p}=0,018)$. Demnach bekamen Männer zum GRAS-Untersuchungszeitpunkt eine signifikant höhere antipsychotische Tagesdosis als Frauen. Abbildung 4 stellt die Unterschiede zwischen den Stichproben in der mittleren CPÄ-Tagesdosis graphisch dar.

Tabelle 3: Deskriptive und inferenzstatistische Werte der mittleren CPÄ-Tagesdosis zum GRASUntersuchungszeitpunkt

\begin{tabular}{|l|c||c|c|}
\hline & gesamt & Männer & Frauen \\
\hline n & 1029 & 689 & 340 \\
\hline Mittelwert & 687,99 & 706,67 & 650,14 \\
\hline Median & 500,00 & 520,00 & 450,00 \\
\hline MD & 437,04 & 440,25 & 427,71 \\
\hline SD & 696,89 & 668,43 & 750,87 \\
\hline Schiefe & 3,25 & 2,64 & 4,15 \\
\hline Kurtosis & 18,26 & 11,76 & 26,85 \\
\hline Minimum & 0 & 0 & 0 \\
\hline Maximum & 7375,00 & 6324,29 & 7375,00 \\
\hline \hline $\begin{array}{l}\text { Mann-Whitney- } \\
\text { U-Test }\end{array}$ & & \multicolumn{2}{|c|}{$\mathrm{z}=-2,361$} \\
\hline
\end{tabular}

MD: mittlere absolute Abweichung vom Median, SD: Standardabweichung

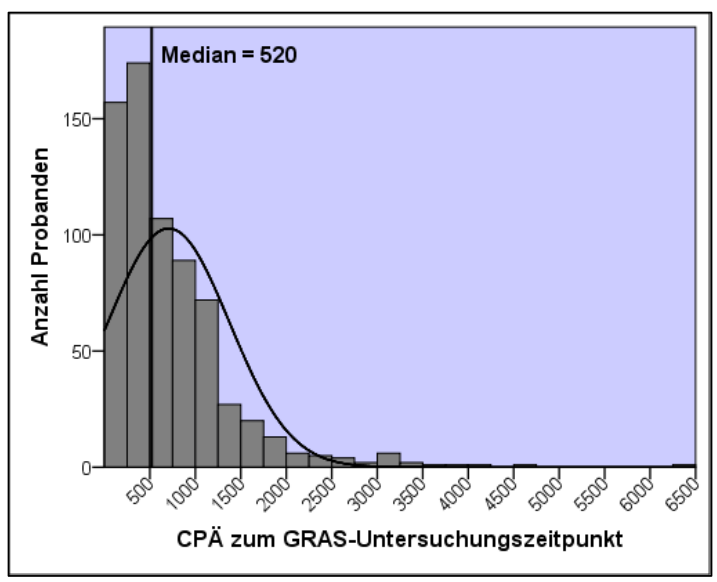

Abbildung 2: Verteilung der antipsychotischen Tagesdosen in Chlorpromazinäquivalenten (CPÄ) zum Zeitpunkt der GRAS-Untersuchung in der Stichprobe der Männer

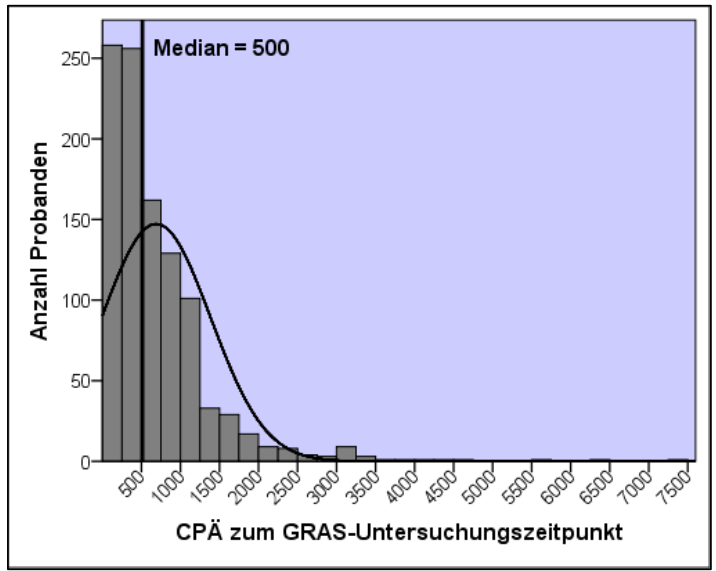

Abbildung 1: Verteilung der antipsychotischen Tagesdosen in Chlorpromazinäquivalenten (CPÄ) zum Zeitpunkt der GRAS-Untersuchung in der Gesamtstichprobe

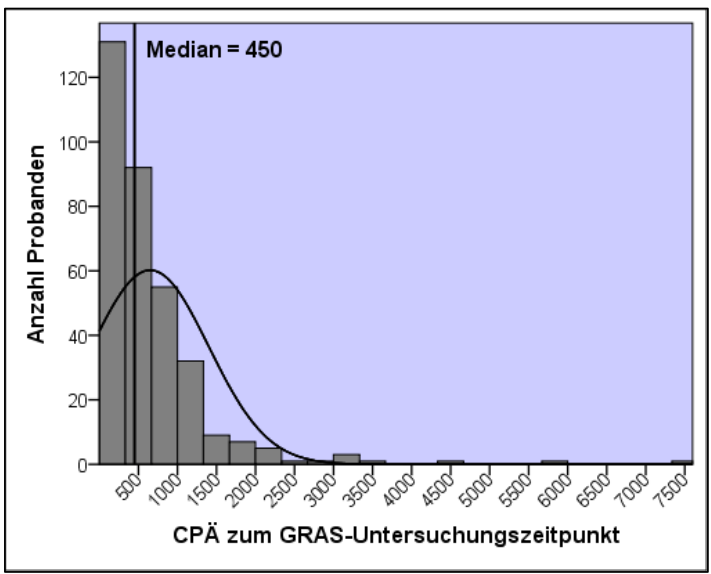

Abbildung 3: Verteilung der antipsychotischen Tagesdosen in Chlorpromazinäquivalenten (CPÄ) zum Zeitpunkt der GRAS-Untersuchung in der Stichprobe der Frauen 
Abbildung 4:

Gegenüberstellung der Medianwerte der antipsychotischen Tagesdosen in Chlorpromazinäquivalenten (CPÄ) in der Gesamt-, Männer- und Frauenstichprobe zum GRASUntersuchungszeitpunkt. Die Streuung ist durch die mittlere absolute Abweichung vom Median verdeutlicht.

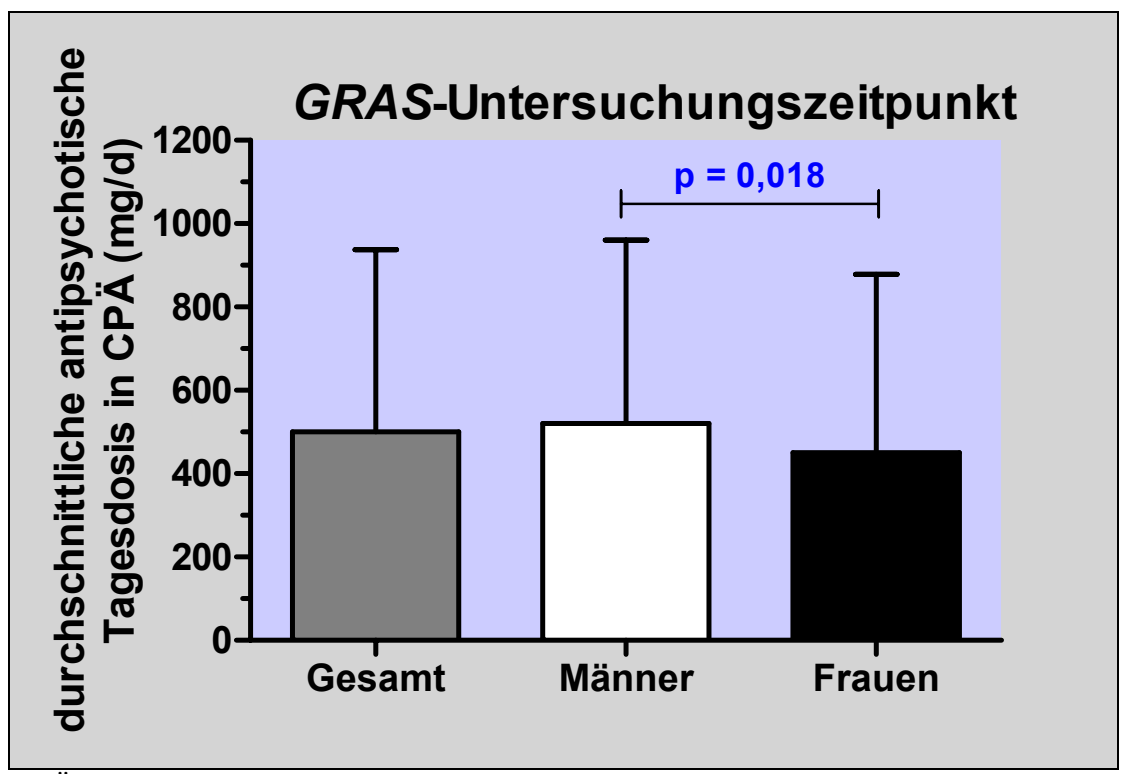

CPÄ: Chlorpromazinäquivalent, p: Irrtumswahrscheinlichkeit

\subsection{Verteilung der antipsychotischen Dosis zum Zeitpunkt der Erstmedikation}

Tabelle 4 sowie die Abbildungen 5 bis 7 geben Auskunft über die Verteilung der CPÄ bei Erstmedikation in den entsprechenden Stichproben. In der Gesamtstichprobe liegt die zentrale Tendenz bei 300mg $\pm 301 \mathrm{mg}$, bei den Männern bei 300mg $\pm 318 \mathrm{mg}$ und bei den Frauen bei 300mg $\pm 266 \mathrm{mg}$. Zudem sind in der Stichprobe der Männer der Mittelwert, die mittlere absolute Abweichung vom Median, die Standardabweichung, die Rechtsschiefe, die Kurtosis und das Maximum jeweils größer als in der Gesamt- und Frauenstichprobe.

Hypothese 2 postuliert einen antipsychotischen Dosisunterschied zwischen Männern und Frauen zum Zeitpunkt der Erstmedikation. Nach Prüfung dieser Hypothese mittels U-Test musste sie verworfen werden $(z=-1,320 ; p=0,187)$. Männer und Frauen der in dieser Arbeit untersuchten Stichprobe unterschieden sich hinsichtlich der Dosishöhe zum Zeitpunkt der Erstmedikation nicht signifikant voneinander. Die Ergebnisse aus Tabelle 4 sind in Abbildung 8 graphisch verdeutlicht. 
Tabelle 4: Deskriptive und inferenzstatistische Werte zur mittleren CPÄ-Dosis zum GRASUntersuchungszeitpunkt

\begin{tabular}{|l|l||l|l|}
\hline & gesamt & Männer & Frauen \\
\hline $\mathbf{n}$ & 841 & 565 & 276 \\
\hline Mittelwert & 453,99 & 476,05 & 408,82 \\
\hline Median & 300,00 & 300,00 & 300,00 \\
\hline MD & 300,74 & 317,62 & 266,20 \\
\hline SD & 595,73 & 664,13 & 420,00 \\
\hline Schiefe & 7,35 & 7,51 & 2,76 \\
\hline Kurtosis & 91,07 & 85,98 & 11,38 \\
\hline Minimum & 0,00 & 0,00 & 0,00 \\
\hline Maximum & 9365,00 & 9365,00 & 3212,00 \\
\hline \hline $\begin{array}{l}\text { Mann-Whitney- } \\
\text { U-Test }\end{array}$ & & $z=-1,320$ \\
\hline
\end{tabular}

MD: mittlere absolute Abweichung vom Median SD: Standardabweichung

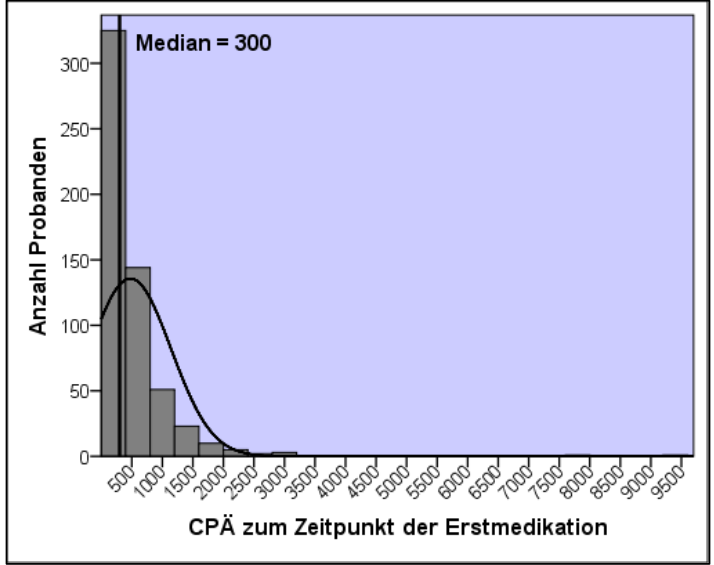

Abbildung 6: Verteilung der antipsychotischen Tagesdosen in Chlorpromazinäquivalenten (CPÄ) zum Zeitpunkt der Erstmedikation in der Stichprobe der Männer

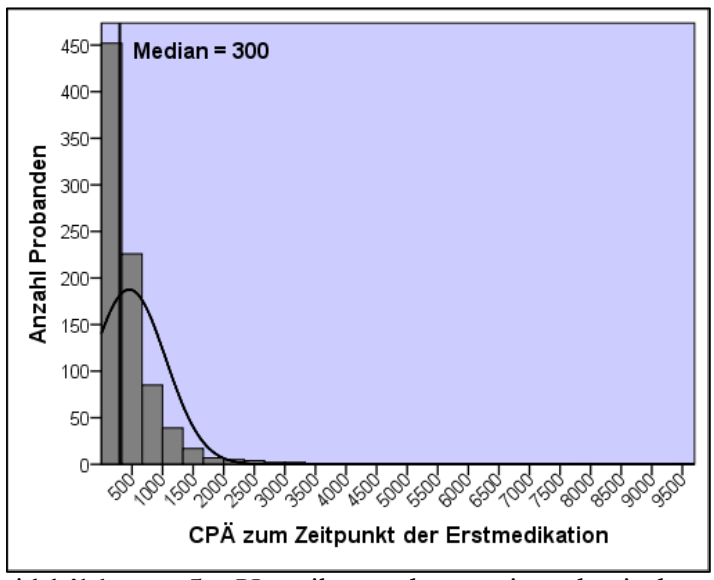

Abbildung 5: Verteilung der antipsychotischen Tagesdosen in Chlorpromazinäquivalenten (CPÄ) zum Zeitpunkt der Erstmedikation in der Gesamtstichprobe

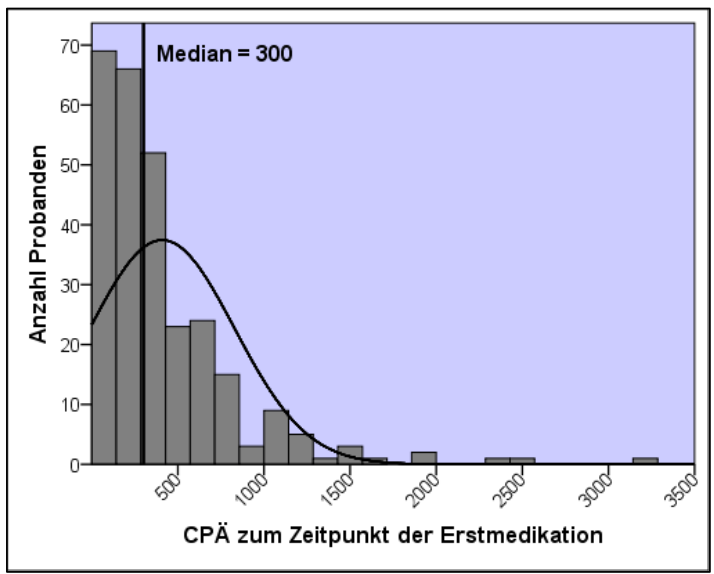

Abbildung 7: Verteilung der antipsychotischen Tagesdosen in Chlorpromazinäquivalenten (CPÄ) zum Zeitpunkt der Erstmedikation in der Stichprobe der Frauen

\section{Abbildung 8:}

Gegenüberstellung der Medianwerte der antipsychotischen Tagesdosen in Chlorpromazinäquivalenten (CPÄ) in der Gesamt-, Männer- und Frauenstichprobe zum Zeitpunkt der Erstmedikation. Die Streuung ist durch die mittlere absolute Abweichung vom Median verdeutlicht.

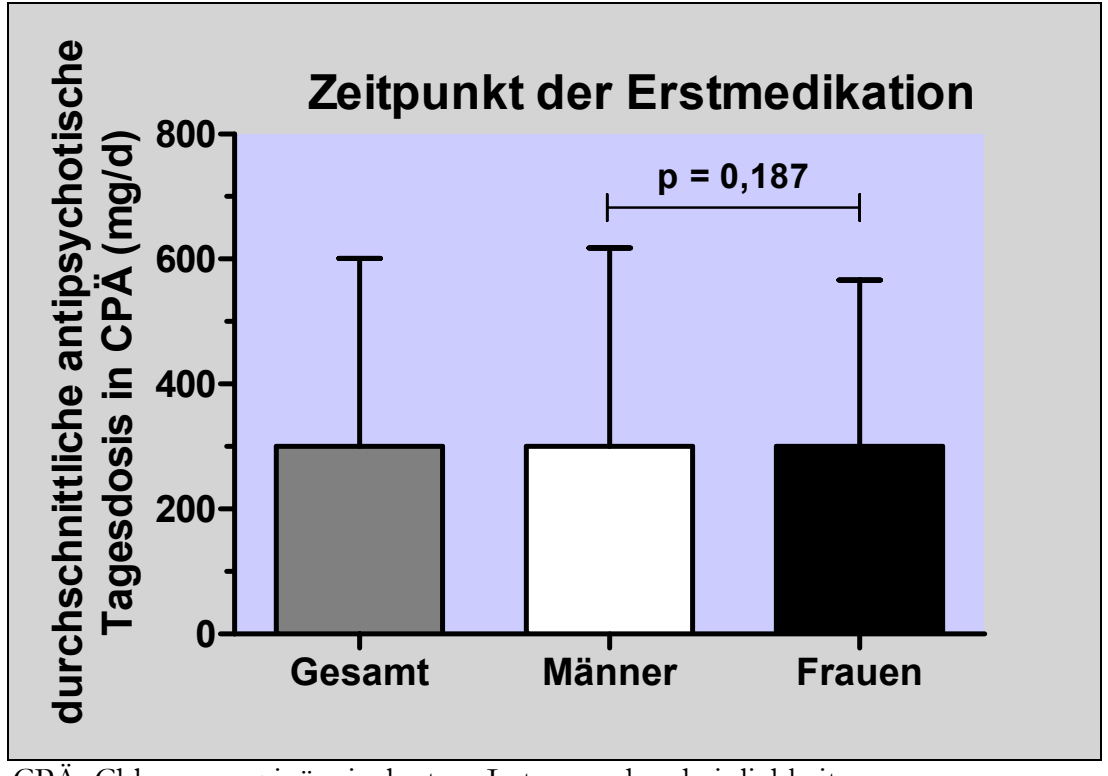

CPÄ: Chlorpromazinäquivalent, p: Irrtumswahrscheinlichkeit 


\subsection{Vergleich der antipsychotischen Dosissteigerung zwischen Männern und Frauen}

Da sich Männer in der antipsychotischen Tagesdosis zum Zeitpunkt der GRASDatenerhebung signifikant von Frauen unterscheiden, nicht jedoch zum Zeitpunkt der Erstmedikation, wurde eine größere antipsychotische Dosissteigerung pro Jahr bei den Männern angenommen. Für diese in Hypothese 3 formulierte Annahme wurde das $\Delta C P \ddot{A} / \mathrm{J}$ errechnet (s. Seite 29 f.). Die betrachteten Stichproben fallen etwas kleiner aus, da nicht von allen Probanden die vollständigen Angaben zur Erstmedikation und/oder der Medikation zum GRASUntersuchungszeitpunkt vorlagen. In Gegenüberstellung der $\Delta \mathrm{CPÄ/J-Werte} \mathrm{der} \mathrm{Männer-} \mathrm{und}$ Frauenstichprobe gibt Tabelle 5 Auskunft über deskriptive und inferenzstatistische Kennwerte. Hypothesenkonform zeigte die Prüfung mittels U-Test eine signifikant höhere Dosissteigerung pro Jahr bei männlichen Probanden, verglichen mit den weiblichen ( $z=-2,095 ; p=0,036)$. Dieses Ergebnis ist in Abbildung 9 graphisch dargestellt.

Tabelle 5: Gegenüberstellung deskriptiver und inferenzstatistischer Werte der durchschnittlichen antipsychotischen Dosissteigerung pro Jahr $(\Delta \mathrm{CP} \ddot{\mathrm{A}} / \mathrm{J})$ in der Männer-, Frauen- und Gesamtstichprobe

\begin{tabular}{|c|c|c|c|c|c|c|c|c|c|c|c|}
\hline & \multirow[b]{2}{*}{ n (\%) } & \multirow[b]{2}{*}{$\mathbf{M}$} & \multirow[b]{2}{*}{ Mdn } & \multirow[b]{2}{*}{ MD } & \multirow[b]{2}{*}{ SD } & \multirow[b]{2}{*}{ Schiefe } & \multirow[b]{2}{*}{$\begin{array}{c}\text { Kurto- } \\
\text { sis }\end{array}$} & \multirow[b]{2}{*}{$\begin{array}{c}\text { Spannwei- } \\
\text { te }\end{array}$} & \multicolumn{3}{|c|}{ Mann-Whitney-U-Test } \\
\hline & & & & & & & & & $\begin{array}{c}\text { Mann- } \\
\text { Whitney- } \\
\text { U }\end{array}$ & mony & $\mathbf{p}$ \\
\hline Männer & $\begin{array}{c}512 \\
(67,99) \\
\end{array}$ & 15,02 & 17,39 & 85,41 & 255,83 & $-5,99$ & 104,39 & $\begin{array}{l}-3799,05 \\
-1817,50 \\
\end{array}$ & \multirow{2}{*}{55863} & \multirow{2}{*}{$-2,095$} & \multirow{2}{*}{0,036} \\
\hline Frauen & $\begin{array}{c}241 \\
(32,01) \\
\end{array}$ & 14,31 & 7,85 & 64,83 & 152,83 & 0,19 & 17,21 & $\begin{array}{c}-875 \\
-933,30 \\
\end{array}$ & & & \\
\hline gesamt & $\begin{array}{c}753 \\
(100)\end{array}$ & 14,79 & 12,24 & 78,93 & 227,88 & $-5,74$ & 113,75 & $\begin{array}{l}-3799,05 \\
-1817,50\end{array}$ & & & \\
\hline
\end{tabular}

M: Mittelwert, Mdn: Median, MD: mittlere absolute Abweichung vom Median; SD: Standardabweichung, p: Irrtumswahrscheinlichkeit, $*:<0,05$

\section{Abbildung 9:}

Gegenüberstellung der Medianwerte der antipsychotischen Dosissteigerung pro Jahr in Chlorpromazinäquivalenten $(\triangle \mathrm{CP} \ddot{\mathrm{A}} / \mathrm{J})$ in der Gesamt-, Männer- und Frauenstichprobe. Die Streuung ist durch die mittlere absolute Abweichung vom Median verdeutlicht.

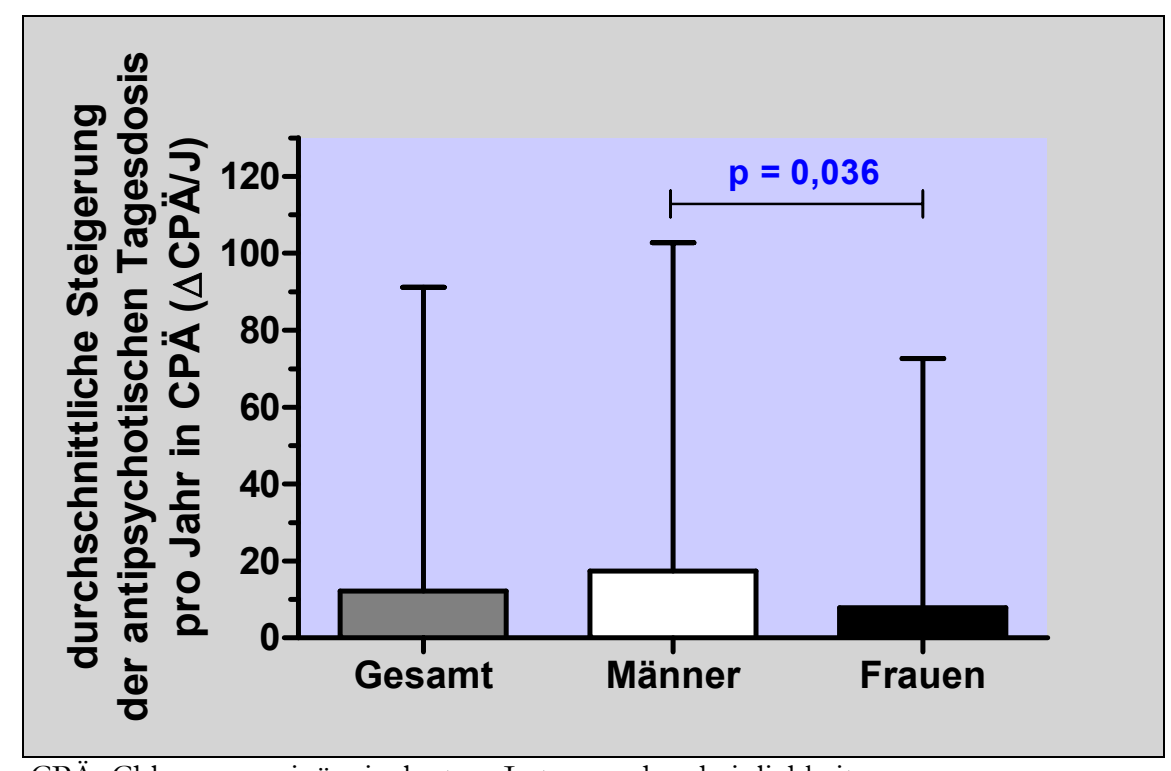

CPÄ: Chlorpromazinäquivalent, p: Irrtumswahrscheinlichkeit 


\subsubsection{Geschlechtsunterschiede in der Ausprägung von Variablen, die mit der Höhe der antipsychotischen Tagesdosis assoziiert sind}

Wie in Kapitel 1.2 und 1.3 beschrieben, unterscheiden sich die Geschlechter auch hinsichtlich der Ausprägung von Variablen, die mit der Quantität der antipsychotischen Tagesdosis assoziiert sind. Diese Variablen sind in Tabelle 6 dargestellt. Für die Gesamtstichprobe sind ausschließlich deskriptive Werte, für die Gruppe der Männer- und Frauenstichprobe deskriptive Werte sowie die inferenzstatistischen Ergebnisse der geprüften Paarkontraste wiedergegeben. Im U-Test zeigte die Stichprobe der Frauen signifikant größere Medianwerte in Alter ( $\mathrm{z}=$ 6,844; $\mathrm{p}<0,001)$, Alter bei Ersterkrankung ( $\mathrm{z}=-5,678 ; \mathrm{p}<0,001)$, Erkerankungsdauer ( $\mathrm{z}=-2,506$; $\mathrm{p}=0,012)$ und Medikationsdauer $(\mathrm{z}=-2,715 ; \mathrm{p}=0,007)$. Demnach waren die Frauen sowohl zum GRAS-Untersuchungszeitpunkt, als auch bei Ersterkrankung, älter als die Männer. Frauen wiesen im Vergleich zu Männern eine längere Erkrankungsdauer auf und nahmen über eine längere Zeit antipsychotische Medikation ein. Sowohl in der Dauer der unbehandelten Erkerankung und Gesamtdauer psychiatrischer Aufenthalte als auch in den Messgrößen psychopathologischer und allgemeiner Beeinträchtigung (PANSS, GAF, CGI) ergaben sich keine signifikanten Gruppenunterschiede.

Tabelle 6: Charakterisierung der Stichproben hinsichtlich modulatorischer Größen der antipsychotischen Medikation

\begin{tabular}{|c|c|c|c|c|c|}
\hline & & $\begin{array}{c}\text { gesamt } \\
\text { Mdn (MD) }\end{array}$ & $\begin{array}{l}\text { Männer } \\
\text { Mdn (MD) }\end{array}$ & $\begin{array}{l}\text { Frauen } \\
\text { Mdn (MD) }\end{array}$ & U-Test \\
\hline \multicolumn{2}{|l|}{$\mathbf{n}$} & 1029 & 689 & 340 & \\
\hline \multicolumn{2}{|l|}{ Alter (a) } & $\begin{array}{c}39,09 \\
(10,313) \\
\end{array}$ & $\begin{array}{c}36,69 \\
(9,959) \\
\end{array}$ & $\begin{array}{c}42,86 \\
(10,144) \\
\end{array}$ & $\begin{array}{l}z=-6,844 \\
p<0,001\end{array}$ \\
\hline \multicolumn{2}{|c|}{ Alter bei Ersterkrankung (a) } & $23,00(6,593)$ & $22(5,700)$ & $26(8,009)$ & $\begin{array}{l}z=-5,678 \\
p<0,001\end{array}$ \\
\hline \multicolumn{2}{|c|}{ Erkrankungsdauer (a) } & $10,89(8,401)$ & $10,16(7,972)$ & $13,02(9,097)$ & $\begin{array}{l}z=-2,506 \\
p=0,012\end{array}$ \\
\hline \multicolumn{2}{|c|}{ Dauer der unbehandelten Erkrankung (a) } & $2,00(3,147)$ & $2,00(3,162)$ & $2,00(3,115)$ & $\begin{array}{l}z=-1,733 \\
p=0,083\end{array}$ \\
\hline \multicolumn{2}{|c|}{$\begin{array}{l}\text { Gesamtdauer psychiatrischer Aufenthalte } \\
\text { (d) }\end{array}$} & $\begin{array}{c}300,00 \\
(558,541) \\
\end{array}$ & $\begin{array}{c}301,00 \\
(584,587) \\
\end{array}$ & $\begin{array}{c}299,00 \\
(506,198) \\
\end{array}$ & $\begin{array}{l}z=-1,140 \\
p=0,254\end{array}$ \\
\hline \multicolumn{2}{|l|}{ GAF } & $45(14,352)$ & $45(13,521)$ & $45(19,093)$ & $\begin{array}{l}z=-0,312 \\
p=0,755\end{array}$ \\
\hline \multicolumn{2}{|l|}{ CGI } & $6(0,846)$ & $6(0,798)$ & $6(0,944)$ & $\begin{array}{l}z=-0,134 \\
p=0,893\end{array}$ \\
\hline \multirow{3}{*}{ PANSS } & positiv & $12(4,917)$ & $12(4,844)$ & $12(5,072)$ & $\begin{array}{l}z=-0,049 \\
p=0,961\end{array}$ \\
\hline & negativ & $17(6,353)$ & $17(6,045)$ & $17(7,000)$ & $\begin{array}{l}z=-0,793 \\
p=0,428\end{array}$ \\
\hline & allgemein & $32(9,359)$ & $32(8,960)$ & $33(10,181)$ & $\begin{array}{l}z=-0,908 \\
p=0,364\end{array}$ \\
\hline \multicolumn{2}{|c|}{ Medikationsdauer (a) } & $9,00(7,638)$ & $8,00(7,195)$ & $11,00(8,485)$ & $\begin{array}{l}z=-2,715 \\
p=0,007\end{array}$ \\
\hline
\end{tabular}




\begin{tabular}{|c|c|c|c|c|c|}
\hline & & $\begin{array}{c}\text { gesamt } \\
\text { Mdn (MD) }\end{array}$ & $\begin{array}{l}\text { Männer } \\
\text { Mdn (MD) }\end{array}$ & $\begin{array}{c}\text { Frauen } \\
\text { Mdn (MD) }\end{array}$ & U-Test \\
\hline & & $\mathrm{n}(\%)$ & $\mathrm{n}(\%)$ & $\mathrm{n}(\%)$ & $\mathrm{X}^{2}$-Test \\
\hline & ambulant & $208(20,2)$ & $144(20,9)$ & $64(18,8)$ & \\
\hline & stationär & $356(34,6)$ & $240(34,8)$ & $116(34,1)$ & $x^{2}=0,792$ \\
\hline Patientenstatus & $\begin{array}{l}\text { betreutes } \\
\text { Wohnen }\end{array}$ & $120(11,7)$ & $86(12,5)$ & $34(10)$ & $p=0,673$ \\
\hline & unbekannt & $345(33,5)$ & $219(31,8)$ & $126(37,1)$ & \\
\hline & oral & $800(77,7)$ & $533(77,4)$ & $267(78,5)$ & \\
\hline & Depot & $78(7,6)$ & $59(8,6)$ & $19(5,6)$ & \\
\hline Untersuchungszeitpunkt & gemischt & $127(12,3)$ & $81(11,8)$ & $46(13,5)$ & $\mathrm{p}=0,351$ \\
\hline & $\begin{array}{l}\text { keine } \\
\text { Medikation }\end{array}$ & $24(2,3)$ & $16(2,3)$ & $8(2,4)$ & \\
\hline & oral & $804(78,1)$ & $540(78,4)$ & $264(77,6)$ & \\
\hline Applikationsart der AP zum & Depot & $89(8,6)$ & $61(8,9)$ & $28(8,2)$ & $x^{2}=0,810$ \\
\hline Zeitpunkt der Erstmedikation & gemischt & $52(5,1)$ & $32(4,6)$ & $20(5,9)$ & $\mathrm{p}=0,847$ \\
\hline & unbekannt & $84(8,2)$ & $56(8,1)$ & $28(8,2)$ & \\
\hline & FGA & $151(14,7)$ & $98(14,2)$ & $53(15,6)$ & \\
\hline Typus des/der applizierten AP & SGA & $542(52,7)$ & $355(51,5)$ & $188(55,29)$ & $x^{2}=2593$ \\
\hline $\begin{array}{l}\text { zum GRAS- } \\
\text { Untersuchungszeitnunkt }\end{array}$ & gemischt & $312(30,3)$ & $220(31,9)$ & $91(26,79)$ & $\mathrm{p}=0,273$ \\
\hline & $\begin{array}{l}\text { keine } \\
\text { Medikation }\end{array}$ & $24(2,3)$ & $16(2,3)$ & $8(2,4)$ & \\
\hline & FGA & $462(44,9)$ & $292(42,4)$ & $170(50)$ & \\
\hline Typus des/der applizierten AP & SGA & $365(35,5)$ & $254(36,9)$ & $111(32,65)$ & $x^{2}=6,533$ \\
\hline $\begin{array}{l}\text { zum Leitpunkt der Erstmealka- } \\
\text { tion }\end{array}$ & gemischt & $118(11,5)$ & $87(12,6)$ & $31(9,12)$ & $p=0,038$ \\
\hline & unbekannt & $84(8,2)$ & $56(8,1)$ & $28(8,24)$ & \\
\hline & $\begin{array}{l}\text { keine } \\
\text { Medikation }\end{array}$ & $24(2,33)$ & $16(2,32)$ & $8(2,35)$ & \\
\hline Summe applizierter AP zum & 1 & $558(54,23)$ & $356(51,67)$ & $200(58,83)$ & $x^{2}=9,861$ \\
\hline $\begin{array}{l}\text { GRAS - } \\
\text { Untercuchungczeitnunkt }\end{array}$ & 2 & $351(34,11)$ & $242(35,12)$ & $109(32,06)$ & $p=0,043$ \\
\hline & 3 & $82(7,97)$ & $64(9,29)$ & $20(5,88)$ & \\
\hline & 4 & $14(1,36)$ & $11(1,60)$ & $3(0,9)$ & \\
\hline & $\begin{array}{l}\text { keine } \\
\text { Medikation }\end{array}$ & $172(16,7)$ & $116(16,8)$ & $56(16,5)$ & \\
\hline Summe applizierter AP zum & 1 & $629(61,1)$ & $408(59,2)$ & $221(65,0)$ & $x^{2}=6,221$ \\
\hline Zeitpunkt der Erstmedikation & 2 & $206(20,0)$ & $152(22,1)$ & $54(15,9)$ & \\
\hline & 3 & $22(2,1)$ & $13(1,9)$ & $9(2,6)$ & \\
\hline & ja & $714(69,4)$ & $524(76,1)$ & $190(55,9)$ & \\
\hline Raucher & nein & $298(29,0)$ & $159(23,1)$ & $139(40,9)$ & $\begin{array}{c}x-45,914 \\
p<0,001\end{array}$ \\
\hline & unbekannt & $17(1,7)$ & $6(1,0)$ & $11(3,2)$ & \\
\hline
\end{tabular}

a: Jahr, CGI: Clinical Global Impression Scale, d: Tag, FGA: Antipsychotikum der ersten Generation, GAF: Global Assessment of Functioning Scale, MD: absolute mittlere Abweichung vom Median; Mdn: Median, PANSS: Positive and Negative Syndrome Scale, SGA: Antipsychotikum der zweiten Generation

Diese Ergebnisse sagen aus, dass Männer und Frauen zum GRAS-Untersuchungszeitpunkt gleich schwer erkrankt waren, was sich im Vergleich der Ergebnissen der Untersuchungsskalen von psychopathologischem Erscheinungsbild, allgemeinem Funktionsniveau, Einschätzung der Krankheitsschwere und auch der Gesamtdauer psychiatrischer Aufenthalte widerspiegelt. Jedoch hatte das Geschlecht einen signifikanten Effekt auf den Typus der verabreichten AP zum Zeitpunkt der Erstmedikation (FGA/SGA/gemischt) $\left(\chi^{2}=6,533 ; \mathrm{p}=0,038\right)$, die Summe der applizierten AP zum GRAS-Untersuchungszeitpunkt ( $\left.\chi^{2}=9,861 ; \mathrm{p}=0,043\right)$ und das Rauchverbalten $\left(\chi^{2}=45,974 ; \mathrm{p}<0,001\right)$. So nahmen Männer zum Zeitpunkt der Erstmedikation zu einem höheren Anteil SGA und eine Kombination aus SGA und FGA ein. Der Anteil der 
Probanden, die mehr als ein AP zum GRAS-Untersuchungszeitpunkt einnahmen, war in der Stichprobe der Männer größer. Auch befanden sich in der Männerstichprobe signifikant mehr Raucher. Das Geschlecht war nicht assoziiert mit den Größen Patientenstatus (ambulant/stationär/betreutes Wohnen), Applikationsart (oral/Depot/gemischt) und Typus der antipsychotischen Therapie zum GRAS-Untersuchungszeitpunkt. Weiterhin bestanden keine signifikanten Unterschiede in der Applikationsart zum Zeitpunkt der Ersterkrankung sowie der Summe der applizierten AP zum Zeitpunkt der Ersterkrankung.

\subsection{Assoziation der antipsychotischen Dosissteigerung mit der Kognition}

Wie auf Seite $10 \mathrm{f}$. beschrieben, scheint die Höhe der antipsychotischen Tagesdosis mit kognitiven Leistungen assoziiert zu sein. Da dargestellt wurde, dass sich die Geschlechter im Hinblick auf Einflussgrößen der Kognition wie auch der antipsychotischen Tagesdosis und Dosissteigerung signifikant unterscheiden, wurden die beiden Gruppen in den folgenden Analysen getrennt betrachtet. In der Stichprobe der Männer und der Frauen wurde ein Mediansplit der Untersuchungsgröße $\Delta C P \ddot{A} / J$ durchgeführt (s. Seite 30 f.). So wurden parallel bei Männern und Frauen diejenigen Patienten mit geringerem $\Delta \mathrm{CP} \ddot{\mathrm{A}} / \mathrm{J}$ als der Medianwert $(\triangle \mathrm{CP} \ddot{\mathrm{A}} / \mathrm{J} \downarrow)$ gegen diejenigen mit größerer antipsychotischer Dosissteigerung pro Medikationsjahr, verglichen mit dem Medianwert, getestet $(\triangle \mathrm{CPÄ} / \mathrm{J} \uparrow)$. Die Schwankungen in der Probandenanzahl zwischen den neuropsychologischen Einzeltests ergaben sich, da schwerkranke Probanden nicht alle Einzeltests bearbeiten konnten.

\subsubsection{Kognitive Gruppenunterschiede in der Stichprobe der Männer}

Der Zentralwert der antipsychotischen Dosissteigerung lag bei den Männern bei 17,39 $\Delta \mathrm{CPÄ/J}$. Tabelle 7 zeigt, dass Männer der $\Delta \mathrm{CPÄ/J \uparrow -Gruppe} \mathrm{im} \mathrm{Mann-Whitney-U-Test} \mathrm{in}$ den beiden kognitiven Einzeltests Alertness ( $\mathrm{z}=-0,237 ; \mathrm{p}=0,042)$ und ZST $(\mathrm{z}=-2,386 ; \mathrm{p}=0,017)$ signifikant besser waren, als die Männer der $\Delta \mathrm{CP} \ddot{A} / \mathrm{J} \downarrow$-Gruppe. In keinem neuropsychologischen Test war die $\Delta \mathrm{CPÄ/J \uparrow -Gruppe} \mathrm{überlegen.} \mathrm{Die} \mathrm{Testung} \mathrm{ergab} \mathrm{keine} \mathrm{signifikanten} \mathrm{Un-}$ terschiede zwischen den Gruppen in den kognitiven Tests BZT, Dotting, LPS3, Tapping, TMTa, TMTb und dem neuropsychologischen Summenwert.

Die neuropsychologischen Testunterschiede sind in der Abbildung 10 verdeutlicht. 
Tabelle 7: Unterschiede kognitiver Leistung zwischen den $\Delta \mathrm{CPÄ/J-Gruppen} \mathrm{in} \mathrm{der} \mathrm{Stichprobe} \mathrm{der} \mathrm{Männer}$

\begin{tabular}{|c|c|c|c|c|c|c|}
\hline $\begin{array}{c}\text { neuropsychologischer } \\
\text { Test }\end{array}$ & $\begin{array}{l}\mathrm{A}=\Delta \mathrm{CPÄ} / J \downarrow(n) \\
\mathrm{B}=\Delta \mathrm{CPÄ} / J \uparrow(\mathrm{n})\end{array}$ & $\begin{array}{l}\text { Mdn (z- } \\
\text { Wert) }\end{array}$ & MD & $\begin{array}{c}\text { Mann- } \\
\text { Whitney-U }\end{array}$ & $\mathbf{z}$ & $\mathbf{p}$ \\
\hline Alertness & $\begin{array}{l}\text { A }(245) \\
\text { B }(244)\end{array}$ & $\begin{array}{c}0,156 \\
-0,114\end{array}$ & $\begin{array}{l}0,817 \\
0,755\end{array}$ & 26707,50 & $-2,037$ & 0,042 \\
\hline BZT & $\begin{array}{l}\text { A }(236) \\
B(225)\end{array}$ & $\begin{array}{l}0,149 \\
-0,121\end{array}$ & $\begin{array}{l}0,807 \\
0,764\end{array}$ & 24539,50 & $-1,412$ & 0,158 \\
\hline Dotting & $\begin{array}{l}\text { A (252) } \\
\text { B }(251)\end{array}$ & $\begin{array}{c}0,173 \\
-0,032\end{array}$ & $\begin{array}{l}0,860 \\
0,752\end{array}$ & 29220,00 & $-1,477$ & 0,140 \\
\hline LPS3 & $\begin{array}{l}\text { A (246) } \\
\text { B (245) }\end{array}$ & $\begin{array}{c}0,104 \\
-0,085 \\
\end{array}$ & $\begin{array}{l}0,760 \\
0,803\end{array}$ & 29105,00 & $-0,656$ & 0,512 \\
\hline MWTB & $\begin{array}{ll}\text { A } & (244) \\
B & (242)\end{array}$ & $\begin{array}{c}0,038 \\
-0,038\end{array}$ & $\begin{array}{l}0,830 \\
0,735\end{array}$ & 28430,50 & $-0,707$ & 0,479 \\
\hline Tapping & $\begin{array}{l}\text { A (251) } \\
\text { B (250) }\end{array}$ & $\begin{array}{c}0,186 \\
-0,118 \\
\end{array}$ & $\begin{array}{l}0,802 \\
0,753 \\
\end{array}$ & 28405,00 & $-1,835$ & 0,067 \\
\hline TMTa & $\begin{array}{ll}\text { A } & (253) \\
\text { B } & (248)\end{array}$ & $\begin{array}{c}0,091 \\
-0,032\end{array}$ & $\begin{array}{l}0,796 \\
0,781\end{array}$ & 29100,50 & $-1,402$ & 0,161 \\
\hline TMTb & $\begin{array}{l}\text { A (237) } \\
\text { B (231) }\end{array}$ & $\begin{array}{c}0,056 \\
-0,056\end{array}$ & $\begin{array}{l}0,792 \\
0,754\end{array}$ & 26578,50 & $-0,544$ & 0,587 \\
\hline VLMT & $\begin{array}{l}\text { A (243) } \\
\text { B }(241)\end{array}$ & $\begin{array}{c}0,117 \\
-0,052 \\
\end{array}$ & $\begin{array}{l}0,781 \\
0,739 \\
\end{array}$ & 26271,50 & $-1,957$ & 0,050 \\
\hline ZST & $\begin{array}{l}\text { A (248) } \\
\text { B }(250)\end{array}$ & $\begin{array}{c}0,142 \\
-0,077\end{array}$ & $\begin{array}{l}0,789 \\
0,712\end{array}$ & 27170,50 & $-2,386$ & 0,017 \\
\hline Summenwert & $\begin{array}{l}A \quad(211) \\
B \quad(201)\end{array}$ & $\begin{array}{c}0,144 \\
-0,034\end{array}$ & $\begin{array}{l}0,786 \\
0,728\end{array}$ & 19034,00 & $-1,797$ & 0,072 \\
\hline
\end{tabular}

BZT: Buchstaben-Zahlen-Test, LPS3: Leistungsprüfsystem Subtest 3, MD: mittlere absolute Abweichung vom Median, Mdn: Median, MWTB: Mehrfach-Wortschatz-Intelligenztest, TMTa/b: Trail Making Test a/b, VLMT: Verbaler Lern- und Merkfähigkeitstest, ZST: Zahlen- Symbol-Test, $\Delta$ CPÄ/J $\downarrow$ bzw. $\uparrow:$ weniger bzw. mehr Steigerung der antipsychotischen Tagesdosis pro Jahr als Medianwert

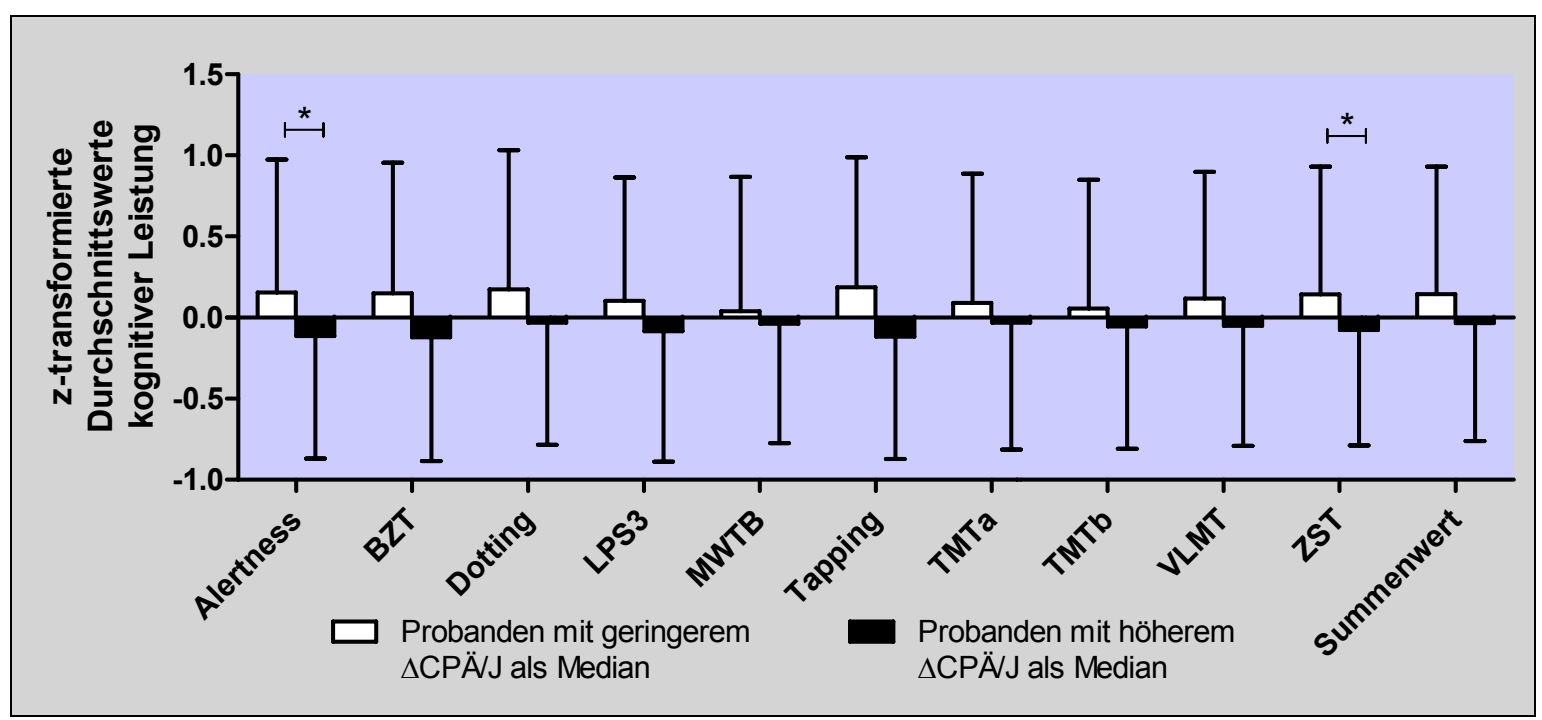

BZT: Buchstaben-Zahlen-Test, LPS3: Leistungsprüfsystem Subtest 3, MD: MWTB: Mehrfach-WortschatzIntelligenztest, TMTa/b: Trail Making Test a/b, VLMT: Verbaler Lern- und Merkfähigkeitstest, ZST: Zahlen-

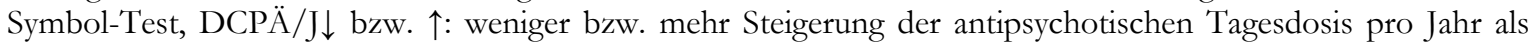
Medianwert, *: $\mathrm{p} \leq 0,05$

Abbildung 10: Neuropsychologische Testunterschiede zwischen den $\Delta \mathrm{CPÄ/J-Gruppen} \mathrm{in} \mathrm{der} \mathrm{Stichprobe} \mathrm{der}$ Männer 
In der Männerstichprobe der vorliegenden Studie konnte die Hypothese 4, welche eine größere kognitive Beeinträchtigung in der $\Delta \mathrm{CP} \ddot{A} / \mathrm{J} \uparrow-G r u p p e$ als in der CPÄ $\downarrow / J$-Gruppe annahm, somit lediglich für die neuropsychologischen Tests Alertness und ZST als bewiesen gelten. Unter Berücksichtigung der Bonferroni-Korrektur ergaben sich keine signifikanten Unterschiede.

\subsection{2 $\triangle \mathrm{CPÄ/J-Gruppenunterschiede} \mathrm{kognitionsmodulierender} \mathrm{Variab-}$ len in der Stichprobe der Männer}

Signifikante Gruppenunterschiede zwischen den beiden $\Delta C P A ̈ / J-G r u p p e n$ in der Männerstichprobe ergab der U-Test bei der Testung von Alter bei Ersterkrankung $(\mathrm{z}=-2,351 ; \mathrm{p}=0,019)$ sowie der Gesamtdauer psychiatrischer Aufenthalte ( $\mathrm{z}=-2,689 ; \mathrm{p}=0,007)$. Somit waren Männer der $\Delta \mathrm{CP} \ddot{\mathrm{A}} / \mathrm{J} \uparrow$ zum Zeitpunkt der Ersterkrankung jünger und zeigten eine längere kumulative Dauer psychiatrischer Aufenthalte. Zudem war die $\Delta \mathrm{CP} \ddot{A} / \mathrm{J} \uparrow$-Gruppe jünger bei der $G R A S$ Untersuchung, länger an Schizophrenie erkrankt, hatte eine längere Dauer der unbehandelten Erkrankung und zeigte eine längere Medikationsdauer, wobei diese Gruppenunterschiede keine Signifikanz erreichten.

Die Zugehörigkeit zu der $\Delta C P \ddot{A} / \mathrm{J} \uparrow$-Gruppe war signifikant mit den Größen Patientenstatus $\left(\chi^{2}\right.$ =7,459; $\mathrm{p}=0,024)$, Typus des applizierten AP zum Untersuchungszeitpunkt $\left(\chi^{2}=33,876 ; \mathrm{p}<0,001\right)$, Summe der applizierten AP zum GRAS-Untersuchungszeitpunkt ( $\left.\chi^{2}=57,974 ; \mathrm{p}<0,001\right)$, Summe der applizierten AP zum Zeitpunkt der Erstmedikation $\left(\chi^{2}=10,547 ; \mathrm{p}=0,014\right)$ sowie antipsychotische Gesamttagesdosis über $1000 \mathrm{CP} \ddot{A} / d\left(\chi^{2}=91,080 ; \mathrm{p}<0,001\right)$ assoziiert. In der $\Delta \mathrm{CP} \ddot{A} / \mathrm{J} \uparrow$-Gruppe befand sich eine höhere Anzahl stationär behandelter Probanden und solcher, die in betreuten Wohneinrichtungen lebten, wohingegen sich in der $\Delta \mathrm{CPÄ/J \downarrow -Gruppe} \mathrm{mehr} \mathrm{ambulante} \mathrm{Pro-}$ banden befanden. Die $\Delta \mathrm{CP} \ddot{A} / \mathrm{J} \uparrow-G r u p p e$ zeigte einen doppelt so hohen Anteil an Probanden, die eine Kombination aus FGA und SGA bekamen. Der Anteil derjenigen, die zum GRASUntersuchungszeitpunkt mehr als ein AP bekamen, war in der $\Delta \mathrm{CPÄ/J} \uparrow$-Gruppe ebenfalls höher. Zum Zeitpunkt der Erstmedikation bekamen mehr Probanden in der $\Delta \mathrm{CP} \ddot{A} / \mathrm{J} \downarrow-$ Gruppe mehr als $1 \mathrm{AP}$ als in der $\Delta \mathrm{CP} \ddot{\mathrm{A}} / \mathrm{J} \uparrow$-Gruppe. Keine signifikanten Unterschiede ergaben sich in der Testung auf Gruppenunterschiede hinsichtlich des Typus des applizierten AP zum Zeitpunkt der Erstmedikation und des Rauchverbaltens. Tabelle 8 stellt die deskriptiven und inferenzstatistischen Ergebnisse zusammen. Zum Zeitpunkt der GRAS-Untersuchung wurden den Männern signifikant mehr AP verabreicht als zum Zeitpunkt der Erstmedikation. Dieses Ergebnis ist in Tabelle 9 zusammengefasst. 
Tabelle 8: Deskriptive und inferenzstatistische Unterschiede kognitionsmodulierender Variablen zwischen den $\Delta C P \ddot{A} / \mathrm{J}-G r u p p e n$ in der Stichprobe der Männer

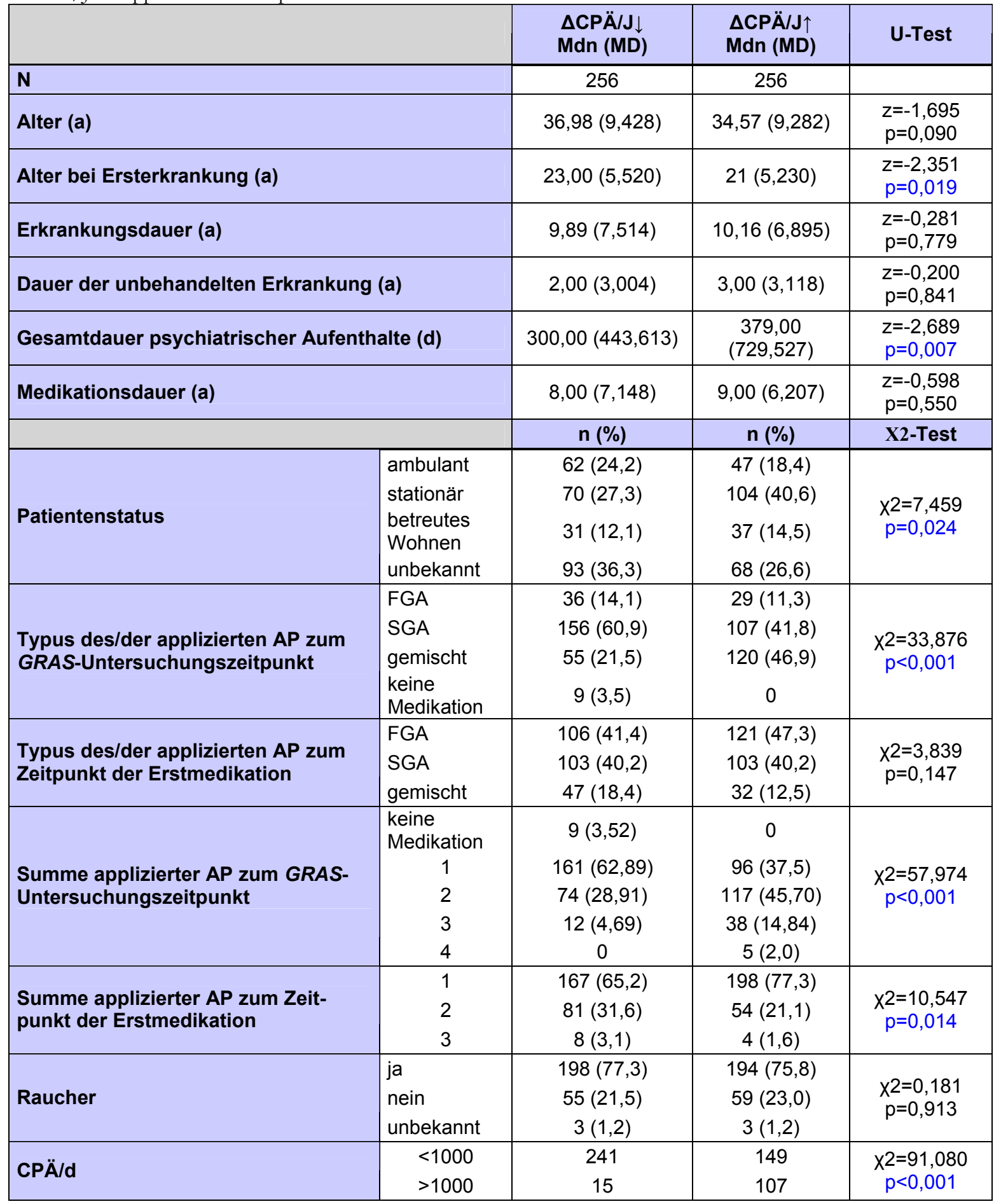

a: Jahr, CGI: Clinical Global Impression Scale, d: Tag, FGA: Antipsychotikum der ersten Generation, GAF: Global Assessment of Functioning Scale, MD: absolute mittlere Abweichung vom Median; Mdn: Median, p: Irrtumswahrscheinlichkeit, PANSS: Positive and Negative Syndrome Scale, SGA: Antipsychotikum der zweiten Generation

Tabelle 9: Anzahl der applizierten AP zum GRAS-Untersuchungszeitpunkt und zum Zeitpunkt der Erstmedikation in der Stichprobe der Männer

\begin{tabular}{|c|c|c|c|c|}
\hline & & \begin{tabular}{|c|} 
GRAS-Untersuchungszeitpunkt \\
$\mathrm{n}(\%)$
\end{tabular} & $\begin{array}{c}\text { Zeitpunkt der Erstmedikation } \\
\mathrm{n}(\%)\end{array}$ & $\chi 2$-Test \\
\hline \multirow{5}{*}{$\begin{array}{l}\text { Anzahl } \\
\text { applizierter } \\
\text { AP }\end{array}$} & 0 & $9(1,8)$ & $0(0,0)$ & \multirow{5}{*}{$\begin{array}{c}x^{2}=44,71 \\
p<0,001\end{array}$} \\
\hline & 1 & $257(50,2)$ & $365(71,3)$ & \\
\hline & 2 & $191(37,3)$ & $135(26,4)$ & \\
\hline & 3 & $509,8)$ & $12(2,3)$ & \\
\hline & 4 & $5(1,0)$ & $0(0,0)$ & \\
\hline
\end{tabular}

AP: Antipsychotika, p: Irrtumswahrscheinlichkeit 


\subsubsection{Kognitive Gruppenunterschiede in der Stichprobe der Frauen}

Der Mediansplit des $\Delta \mathrm{CP} \ddot{\mathrm{A}} / \mathrm{J}$ wurde in der Frauenstichprobe bei dem Wert 7,85 $\Delta \mathrm{CP} \ddot{\mathrm{A}} / \mathrm{J}$ durchgeführt. Wie in der Stichprobe der Männer zeigten Frauen mit einem $\Delta \mathrm{CPÄ/J} \mathrm{kleiner}$ der Mediangruppe im Mann-Whitney-U-Test bessere Ergebnisse in den kognitiven Tests, als Frauen mit $\Delta \mathrm{CP} \ddot{\mathrm{I}} / \mathrm{J}$-Werten größer der Mediangruppe. Tabelle 10 verdeutlicht, dass zwischen den beiden Dosissteigerungsgruppen signifikante Unterschiede in den Tests Alertness ( $\mathrm{z}=$ 2,208; $\mathrm{p}=0,027)$, BZT ( $\mathrm{z}=-3,120 ; \mathrm{p}=0,002)$, Dotting $(\mathrm{z}=-2,471 ; \mathrm{p}=0,013)$ LPS3 $(\mathrm{z}=-2,458$; $\mathrm{p}=0,014)$, Tapping $(\mathrm{z}=-0,683 ; \mathrm{p}=0,007), \operatorname{TMTa}(\mathrm{z}=-2,621 ; \mathrm{p}=0,009), \operatorname{TMTb} \quad(\mathrm{z}=-2,958$; $\mathrm{p}=0,003), \operatorname{VLMT}(\mathrm{z}=-3,313 ; \mathrm{p}<0,001), Z S T(\mathrm{z}=-2,696 ; \mathrm{p}=0,007)$ und dem neuropsychologischen Summenwert $(\mathrm{z}=-3,568 ; \mathrm{p}<0,001)$ bestehen. Keinen signifikanten Unterschied zwischen den Gruppen ergab die Untersuchung des Tests MWTB ( $z=-0,035 ; \mathrm{p}=0,972)$. Somit war die $\Delta \mathrm{CP} \ddot{\mathrm{A}} / \mathrm{J} \downarrow$-Gruppe bis auf die Testung der kristallinen Intelligenz (MWTB) in allen kognitiven Leistungen signifikant besser als die $\Delta \mathrm{CPÄ/J \uparrow -Gruppe.} \mathrm{Abbildung} 11$ stellt die Ergebnisse im Säulendiagramm dar.

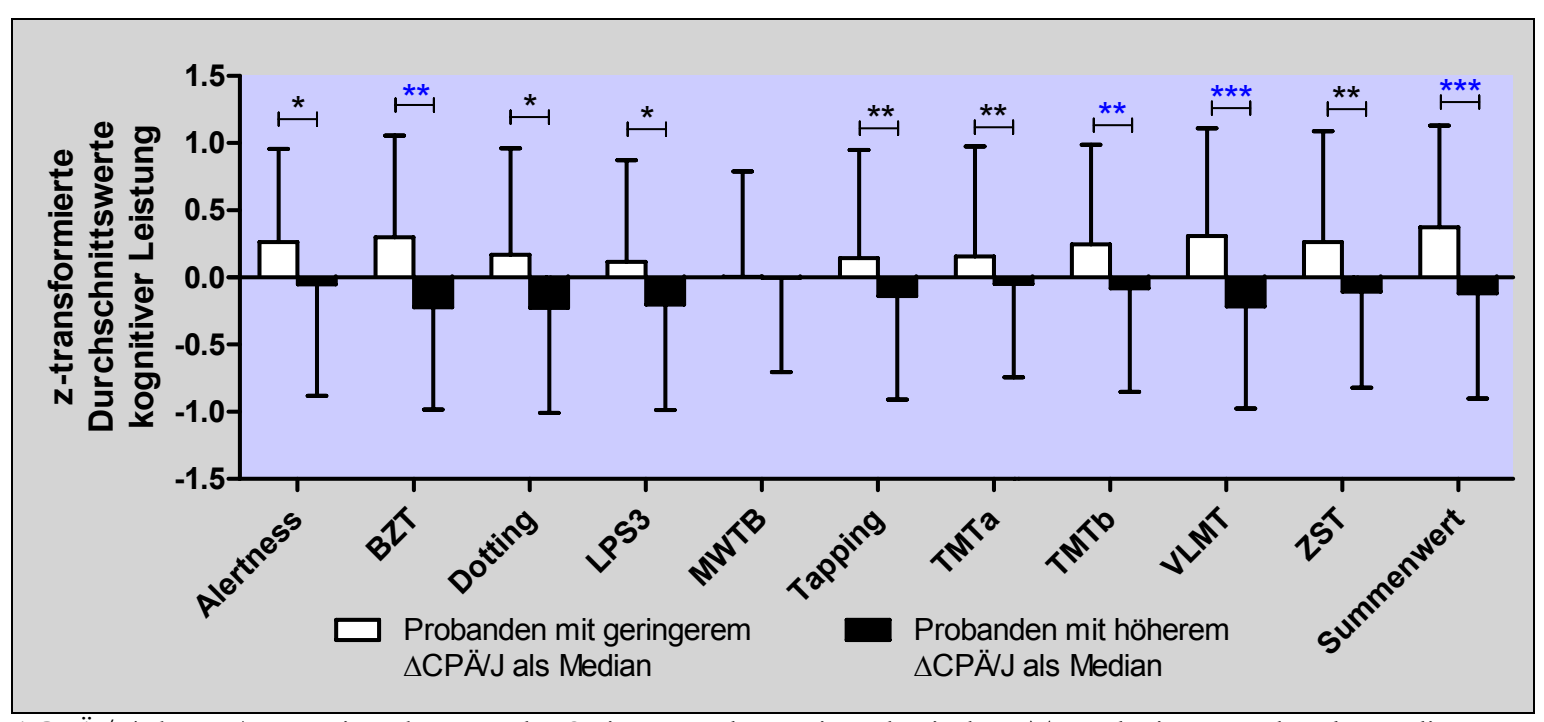

$\triangle \mathrm{CPÄ} / \mathrm{J} \downarrow$ bzw. $\uparrow:$ weniger bzw. mehr Steigerung der antipsychotischen Tagesdosis pro Jahr als Medianwert, BZT: Buchstaben-Zahlen-Test, LPS3: Leistungsprüfsystem Subtest 3, MWTB: Mehrfach-WortschatzIntelligenztest, TMTa/b: Trail Making Test a/b, VLMT: Verbaler Lern- und Merkfähigkeitstest, ZST: ZahlenSymbol-Test, *: $\mathrm{p} \leq 0,05, * *: \mathrm{p} \leq 0,01, * * *: \mathrm{p} \leq 0,001, * * / * * *$ : Signifikanz nach Bonferroni-Korrektur

Abbildung 11: Neuropsychologische Testunterschiede zwischen den $\Delta \mathrm{CPÄ/J-Gruppen} \mathrm{in} \mathrm{der} \mathrm{Stichprobe} \mathrm{der}$ Frauen 


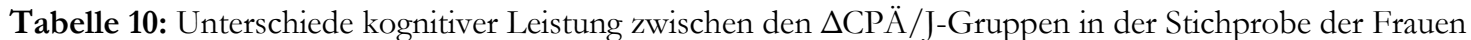

\begin{tabular}{|c|c|c|c|c|c|c|}
\hline $\begin{array}{c}\text { neuropsychologischer } \\
\text { Test }\end{array}$ & $\begin{array}{l}\mathrm{A}=\Delta \mathrm{CPÄ} / \mathrm{J} \downarrow \text { (n) } \\
\mathrm{B}=\Delta \mathrm{CPÄ} / \mathrm{J} \uparrow(\mathrm{n})\end{array}$ & $\begin{array}{l}\text { Mdn (z- } \\
\text { Wert) }\end{array}$ & MD & $\begin{array}{c}\text { Mann- } \\
\text { Whitney-U }\end{array}$ & $\mathbf{Z}$ & $\mathbf{p}$ \\
\hline Alertness & $\begin{array}{l}\text { A (115) } \\
B(117)\end{array}$ & $\begin{array}{c}0,264 \\
-0,051 \\
\end{array}$ & $\begin{array}{l}0,693 \\
0,831\end{array}$ & 5599,00 & $-2,208$ & 0,027 \\
\hline BZT & $\begin{array}{l}\text { A (111) } \\
\text { B (113) }\end{array}$ & $\begin{array}{l}0,300 \\
-0,221\end{array}$ & $\begin{array}{l}0,755 \\
0,763\end{array}$ & 4764,00 & $-3,120$ & $0,002^{*}$ \\
\hline Dotting & $\begin{array}{l}A(112) \\
B(118)\end{array}$ & $\begin{array}{c}0,169 \\
-0,227\end{array}$ & $\begin{array}{l}0,792 \\
0,782\end{array}$ & 5362,00 & $-2,471$ & 0,013 \\
\hline LPS3 & $\begin{array}{l}A(114) \\
B(115)\end{array}$ & $\begin{array}{c}0,117 \\
-0,200\end{array}$ & $\begin{array}{l}0,757 \\
0,785\end{array}$ & 5324,50 & $-2,458$ & 0,014 \\
\hline MWTB & $\begin{array}{l}A(116) \\
B(119)\end{array}$ & $\begin{array}{c}0,004 \\
-0,004\end{array}$ & $\begin{array}{l}0,786 \\
0,699\end{array}$ & 6884,00 & $-0,035$ & 0,972 \\
\hline Tapping & $\begin{array}{l}A(112) \\
B(117)\end{array}$ & $\begin{array}{c}0,143 \\
-0,139\end{array}$ & $\begin{array}{l}0,805 \\
0,770\end{array}$ & 5208,50 & $-2,683$ & 0,007 \\
\hline TMTa & $\begin{array}{l}A(118) \\
B(118)\end{array}$ & $\begin{array}{c}0,157 \\
-0,050\end{array}$ & $\begin{array}{l}0,820 \\
0,693\end{array}$ & 5588,00 & $-2,621$ & 0,009 \\
\hline TMTb & $\begin{array}{l}\text { A (112) } \\
B(110)\end{array}$ & $\begin{array}{c}0,245 \\
-0,082 \\
\end{array}$ & $\begin{array}{l}0,743 \\
0,770 \\
\end{array}$ & 4744,50 & $-2,958$ & $0,003^{*}$ \\
\hline VLMT & $\begin{array}{l}A(115) \\
B(116)\end{array}$ & $\begin{array}{c}0,308 \\
-0,216\end{array}$ & $\begin{array}{l}0,803 \\
0,759\end{array}$ & 4988,00 & $-3,313$ & $<0,001^{*}$ \\
\hline ZST & $\begin{array}{l}A(116) \\
B(118)\end{array}$ & $\begin{array}{c}0,264 \\
-0,106\end{array}$ & $\begin{array}{l}0,824 \\
0,716\end{array}$ & 5448,50 & $-2,696$ & 0,007 \\
\hline Summenwert & $\begin{array}{l}\text { A (97) } \\
\text { B (99) }\end{array}$ & $\begin{array}{l}0,374 \\
-0,119\end{array}$ & $\begin{array}{l}0,758 \\
0,783\end{array}$ & 3385,00 & $-3,568$ & $<0,001^{*}$ \\
\hline
\end{tabular}

$\Delta \mathrm{CPÄ} / \mathrm{J} \downarrow$ bzw. $\uparrow:$ weniger bzw. mehr Steigerung der antipsychotischen Tagesdosis pro Jahr als Medianwert, Mdn: Median, MD: mittlere absolute Abweichung vom Median, BZT: Buchstaben-Zahlen-Test, LPS3: Leistungsprüfsystem Subtest 3, MWTB: Mehrfach-Wortschatz-Intelligenztest, p: Irrtumswahrscheinlichkeit; TMTa/b: Trail Making Test a/b, VLMT: Verbaler Lern- und Merkfähigkeitstest, ZST: Zahlen- Symbol-Test, *: Signifikanz nach Bonferroni-Korrektur

Die Ergebnisse verdeutlichen, dass die Hypothese 4 in der Stichprobe der Frauen bis auf eine Ausnahme bestätigt werden konnte. Bis auf die kristalline Intelligenz (MWTB) waren die Frauen der $\Delta \mathrm{CPÄ/J \uparrow -Gruppe} \mathrm{in} \mathrm{allen} \mathrm{kognitiven} \mathrm{Tests} \mathrm{der} \mathrm{CPÄ/J \downarrow -Gruppe} \mathrm{statistisch} \mathrm{signi-}$ fikant überlegen.

\subsection{4 $\triangle \mathrm{CPÄ/J-Gruppenunterschiede} \mathrm{kognitionsmodulierender} \mathrm{Variab-}$ len in der Stichprobe der Frauen}

Der U-Test ergab signifikante Gruppenunterschiede zwischen den beiden $\Delta \mathrm{CP} \ddot{A} / \mathrm{J}$-Gruppen in der Stichprobe der Frauen bei Testung der Gesamtdauer psychiatrischer Aufenthalte ( $\mathrm{z}=-3,141$; $\mathrm{p}=0,002)$. So zeigten die Probanden der $\Delta \mathrm{CP} \ddot{A} / \mathrm{J} \uparrow-$ Gruppen eine signifikant längere kumulative Gesamtdauer psychiatrischer Aufenthalte als diejenigen der $\Delta \mathrm{CPÄ/J \downarrow -Gruppe.} \mathrm{Zudem}$ war die $\Delta C P \ddot{A} / \mathrm{J} \uparrow$-Gruppe jünger bei der GRAS-Untersuchung, jünger zum Zeitpunkt der Ersterkrankung, länger an Schizophrenie erkerankt, hatte eine längere Dauer der unbehandelten Erkrankung und zeigte eine kürzere Medikationsdawer, wobei diese Gruppenunterschiede keine Signifikanz erreichten (vgl. Tab. 12). 
Die Zugehörigkeit zu der $\Delta \mathrm{CP} \ddot{A} / \mathrm{J} \uparrow-G r u p p e$ war signifikant mit den Größen Patientenstatus $\left(\chi^{2}=7,200 ; \mathrm{p}=0,027\right)$, Typus des applizierten AP zum GRAS-Untersuchungszeitpunkt $\left(\chi^{2}=15,370\right.$; $\mathrm{p}<0,001)$, Summe der applizierten AP zum GRAS-Untersuchungszeitpunkt $\left(\chi^{2}=28,574 ; \mathrm{p}<0,001\right)$ und zum Zeitpunkt der Erstmedikation $\left(\chi^{2}=7,658 ; \mathrm{p}=0,022\right)$, sowie antipsychotische Gesamttagesdosis über $1000 \mathrm{CP} \ddot{A} / d\left(\chi^{2}=32,145 ; \mathrm{p}<0,001\right)$ assoziiert. In der $\Delta \mathrm{CP} \ddot{A} / \mathrm{J} \uparrow$-Gruppe befand sich eine höhere Anzahl an Probanden, die in betreuten Wohneinrichtungen lebte. Die $\Delta \mathrm{CP} \ddot{\mathrm{A}} / \mathrm{J} \uparrow$ Gruppe zeigte einen mehr als doppelt so hohen Anteil an Probanden, die eine Kombination aus FGA und SGA bekamen. Der Anteil derjenigen, die mehr als ein AP zum GRASUntersuchungszeitpunkt bekamen, war in der $\Delta \mathrm{CPÄ/J \uparrow -Gruppe} \mathrm{ebenfalls} \mathrm{deutlich} \mathrm{höher.}$ Zum Zeitpunkt der Erstmedikation bekamen mehr Probanden in der $\Delta \mathrm{CP} \ddot{A} / \mathrm{J} \downarrow$-Gruppe mehr als $1 \mathrm{AP}$ als in der $\Delta \mathrm{CP} \ddot{\mathrm{A}} / \mathrm{J} \uparrow-$ Gruppe. Keine Unterschiede ergaben sich in der Testung auf Gruppenunterschiede hinsichtlich des Typus des applizierten AP zum Zeitpunkt der Erstmedikation und des Rauchverbaltens. Einen Überblick über die deskriptiven und inferenzstatistischen Ergebnisse gibt Tabelle 12. Zum Zeitpunkt der GRAS-Untersuchung wurden den Frauen signifikant mehr AP verabreicht, als zum Zeitpunkt der Erstmedikation. Dieses Ergebnis ist in Tabelle 11 zusammengefasst.

Tabelle 11: Anzahl der applizierten AP zum GRAS-Untersuchungszeitpunkt und zum Zeitpunkt der Erstmedikation in der Stichprobe der Frauen

\begin{tabular}{|c|c|c|c|c|}
\hline & & $\begin{array}{c}\text { GRAS-Untersuchungszeitpunkt } \\
\mathrm{n}(\%)\end{array}$ & $\begin{array}{c}\text { Zeitpunkt der Erstmedikation } \\
\mathrm{n}(\%)\end{array}$ & $\chi 2$-Test \\
\hline \multirow{5}{*}{$\begin{array}{l}\text { Anzahl } \\
\text { applizierter } \\
\text { AP }\end{array}$} & 0 & $8(3,1)$ & $0(0,0)$ & \multirow{5}{*}{$\begin{array}{c}x^{2}=20,11 \\
p=0,049\end{array}$} \\
\hline & 1 & $144(59,8)$ & $137(56,9)$ & \\
\hline & 2 & $75(31,1)$ & $97(40,3)$ & \\
\hline & 3 & $11(4,6)$ & $7(2,9)$ & \\
\hline & 4 & $3(1,2)$ & $0(0,0)$ & \\
\hline
\end{tabular}

AP: Antipsychotika, p: Irrtumswahrscheinlichkeit

Tabelle 12: Deskriptive und inferenzstatistische Unterschiede kognitionsmodulierender Variablen zwischen den $\Delta \mathrm{CPÄ/J}$-Gruppen in der Stichprobe der Frauen

\begin{tabular}{|c|c|c|c|}
\hline & $\begin{array}{c}\Delta \mathrm{CPÄ} / \mathrm{J} \downarrow \\
\operatorname{Mdn}(\mathrm{MD})\end{array}$ & $\begin{array}{c}\Delta \mathrm{CPÄ} / \mathrm{J} \uparrow \\
\operatorname{Mdn}(\mathrm{MD})\end{array}$ & U-Test \\
\hline $\mathbf{n}$ & 120 & 121 & \\
\hline Alter (a) & $43,28(10,340)$ & $41,37(9,213)$ & $\begin{array}{c}z=-0,668 \\
p=0,504\end{array}$ \\
\hline Alter bei Ersterkrankung (a) & $27(7,143)$ & $25(7,132)$ & $\begin{array}{l}z=-1,010 \\
p=0,313\end{array}$ \\
\hline Erkrankungsdauer (a) & $13,73(8,666)$ & $12,61(8,484)$ & $\begin{array}{l}z=-0,245 \\
p=0,806\end{array}$ \\
\hline Dauer der unbehandelten Erkrankung (a) & $2,00(3,472)$ & $3,00(2,788)$ & $\begin{array}{l}z=-0,207 \\
p=0,836\end{array}$ \\
\hline Gesamtdauer psychiatrischer Aufenthalte (d) & $\begin{array}{c}282,00 \\
(284,636)\end{array}$ & $\begin{array}{c}388.00 \\
(782,983)\end{array}$ & $\begin{array}{l}z=-3,141 \\
p=0,002\end{array}$ \\
\hline Medikationsdauer (a) & $12,50(8,283)$ & $12,00(7,983)$ & $\begin{array}{l}z=-0,150 \\
p=0,881\end{array}$ \\
\hline
\end{tabular}




\begin{tabular}{|c|c|c|c|c|}
\hline & & n (\%) & n (\%) & $\chi^{2}$-Test \\
\hline Patientenstatus & $\begin{array}{l}\text { ambulant } \\
\text { stationär } \\
\text { betreutes } \\
\text { Wohnen } \\
\text { unbekannt }\end{array}$ & $\begin{array}{c}33(27,5) \\
34(28,3) \\
7(5,8) \\
46(38,3) \\
\end{array}$ & $\begin{array}{l}13(15,7) \\
35(28,9) \\
16(13,2) \\
51(42,1) \\
\end{array}$ & $\begin{array}{l}x^{2}=7,200 \\
p=0,027\end{array}$ \\
\hline $\begin{array}{l}\text { Typus des/der applizierten AP zum Un- } \\
\text { tersuchungszeitpunkt }\end{array}$ & $\begin{array}{l}\text { FGA } \\
\text { SGA } \\
\text { gemischt } \\
\text { keine Medi- } \\
\text { kation }\end{array}$ & $\begin{array}{c}22(18,3) \\
73(60,8) \\
17(14,2) \\
8(6,7)\end{array}$ & $\begin{array}{c}13(10,7) \\
63(52,1) \\
45(37,2) \\
0\end{array}$ & $\begin{array}{l}x^{2}=15,370 \\
p \leq 0,001\end{array}$ \\
\hline $\begin{array}{l}\text { Typus des/der applizierten AP zum Zeit- } \\
\text { punkt der Erstmedikation }\end{array}$ & $\begin{array}{l}\text { FGA } \\
\text { SGA } \\
\text { gemischt }\end{array}$ & $\begin{array}{c}65(54,2 \\
40(33,3) \\
15(12,5)\end{array}$ & $\begin{array}{c}67(55,4) \\
45(37,2) \\
9(7,4)\end{array}$ & $\begin{array}{l}x^{2}=1,820 \\
p=0,402\end{array}$ \\
\hline $\begin{array}{l}\text { Summe applizierter AP zum Untersu- } \\
\text { chungszeitpunkt }\end{array}$ & $\begin{array}{l}\text { keine Medi- } \\
\text { kation } \\
\qquad \begin{array}{r}1 \\
2 \\
3 \\
4\end{array}\end{array}$ & $\begin{array}{c}8(6,7) \\
85(70,8) \\
26(21,7) \\
1(0,8) \\
0\end{array}$ & $\begin{array}{c}0 \\
59(48,7) \\
49(40,5) \\
10(8,3) \\
3(2,5)\end{array}$ & $\begin{array}{c}x^{2}=28,574 \\
p \leq 0,001\end{array}$ \\
\hline $\begin{array}{l}\text { Summe applizierter AP zum Zeitpunkt } \\
\text { der Erstmedikation }\end{array}$ & $\begin{array}{l}1 \\
2 \\
3\end{array}$ & $\begin{array}{c}86(71,1) \\
27(22,5) \\
7(5,8)\end{array}$ & $\begin{array}{c}102(84,3) \\
18(14,9) \\
1(0,8)\end{array}$ & $\begin{array}{l}x^{2}=7,658 \\
p=0,022\end{array}$ \\
\hline Raucher & $\begin{array}{l}\text { ja } \\
\text { nein } \\
\text { unbekannt }\end{array}$ & $\begin{array}{c}65(54,2) \\
50(41,7) \\
5(4,2)\end{array}$ & $\begin{array}{c}72(59,5) \\
47(38,8) \\
2(1,7)\end{array}$ & $\begin{array}{l}x^{2}=1,732 \\
p=0,421\end{array}$ \\
\hline CPÄ/d & $\begin{array}{l}<1000 \\
>1000\end{array}$ & $\begin{array}{c}119 \\
1\end{array}$ & $\begin{array}{l}90 \\
31\end{array}$ & $\begin{array}{c}x^{2}=32,145 \\
p<0,001\end{array}$ \\
\hline
\end{tabular}

a: Jahr, AP: Antipsychotikum, CGI: Clinical Global Impression Scale, d: Tag, FGA: Antipsychotikum der ersten Generation, GAF: Global Assessment of Functioning Scale, MD: absolute mittlere Abweichung vom Median; Mdn: Median, p: Irrtumswahrscheinlichkeit, PANSS: Positive and Negative Syndrome Scale, SGA: Antipsychotikum der zweiten Generation

\subsection{Assoziation antipsychotischer Dosissteigerung pro Jahr mit}

\section{Kognition unter Berücksichtigung des Alters}

Auf Seite 31 f. wurde beschrieben, dass in der untersuchten Stichprobe eine konsistente Assoziation von Alter mit den kognitiven Einzeltests besteht. Das Alter der Probanden korrelierte konsistent negativ mit den Leistungen aller kognitiven Tests. Es ist bekannt, dass gesunde sowie schizophrene Menschen im Alter schlechtere kognitive Leistungen in Gedächtnis, schlussfolgerndem Denken, Exekutivfunktionen und Wortschatz aufweisen als in jüngerem Lebensalter (Harvey et al. 2010; Salthouse 2010). Deshalb wurden Kovarianzanalysen (ANCOVAs) durchgeführt, um zu prüfen, ob die statistisch signifikanten Unterschiede in einzelnen kognitiven Tests zwischen den jeweils $2 \Delta \mathrm{CPÄ/J-Gruppen} \mathrm{auch} \mathrm{unter} \mathrm{Berücksichtigung}$ des Alters Bestand haben.

In den Tabellen sind für jeden neuropsychologischen Test 3 Ergebnisspalten aufgeführt. Spalte I ist mit „, $\underline{\text { Faktor }}^{“}$ überschrieben. Der Faktor ist die Variable $\Delta \mathrm{CPÄ} / \mathrm{J}$-Gruppe. Die Ergeb- 
niswerte sagen aus, ob die jeweilig $\triangle \mathrm{CP} \ddot{\mathrm{A}} / \mathrm{J}$-Gruppenzugehörigkeit mit den kognitiven Einzeltests assoziiert ist. Spalte II zeigt die Ergebnisse des Rechenmodells, indem sowohl die Asso-

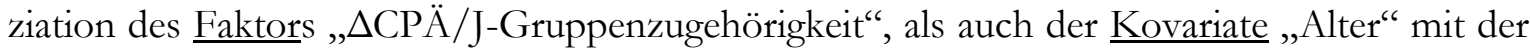
Kognition getestet wurden. Spalte III (Faktor im Modell) zeigt die Ergebnisse der alleinigen Assoziation der jeweiligen $\Delta \mathrm{CP} \ddot{\mathrm{A}} / \mathrm{J}$-Gruppenzugehörigkeit mit der Kognition unter Berücksichtigung des Alters. Diese beschreiben, inwieweit die $\Delta \mathrm{CP} \ddot{\mathrm{A}} / \mathrm{J}$-Gruppenzugehörigkeit unter Berücksichtigung der Störgröße „Alter“ mit kognitiven Leistungen assoziiert ist.

Nachfolgend werden unter Berücksichtigung des Alters die $\Delta$ CPÄ/J-Gruppenunterschiede hinsichtlich der kognitiven Testung in paralleler Betrachtung der beiden Geschlechter dargestellt.

\subsubsection{Unterschiede in der Stichprobe der Männer}

Tabelle 13 zeigt, dass sich die beiden $\Delta \mathrm{CPÄ/J-Gruppen} \mathrm{unter} \mathrm{Berücksichtigung} \mathrm{des} \mathrm{Alters} \mathrm{in}$ deutlich mehr neuropsychologischen Einzeltests unterscheiden, als unter Missachtung dieser Variable (vgl. Tab. 7). So lagen signifikante Unterschiede in den Tests Alertness ( $\mathrm{F}=9,711$; $\mathrm{p}=0,002)$, Tapping $(\mathrm{F}=5,393 ; \mathrm{p}=0,021), \operatorname{TMTa}(\mathrm{F}=4,418 ; \mathrm{p}=0,036), \operatorname{VLMT} \quad(\mathrm{F}=4,676$; $\mathrm{p}=0,031), Z S T(\mathrm{~F}=9,717 ; \mathrm{p}=0,002)$ und des neuropsychologischen Summenwertes $(\mathrm{F}=5,687$; $\mathrm{p}=0,018)$ zwischen den $\Delta \mathrm{CPÄ/J-Gruppen} \mathrm{vor.} \mathrm{Nach} \mathrm{Bonferroni-Korrektur} \mathrm{des} \mathrm{Signifikanz-}$ niveaus unterschieden sich die beiden $\Delta \mathrm{CP} \ddot{\mathrm{A}} / \mathrm{J}-$ Gruppen signifikant in den Tests Alertness sowie ZST.

Tabelle 13: Kennwerte der ANOVA zur Prüfung der Assoziation des Alters mit den $\Delta$ CPÄ/JGruppenunterschieden in neuropsychologischen Tests in der Stichprobe der Männer

\begin{tabular}{|c|c|c|c|c|c|c|c|}
\hline \multirow{2}{*}{ neuropsychologischer Test } & \multirow{2}{*}{$\Delta \mathrm{CPÄ} / \mathbf{J} \downarrow / \Delta \mathrm{CPÄ} / \mathbf{J} \uparrow$} & \multicolumn{2}{|c|}{$\begin{array}{c}\text { I } \\
\text { Faktor }\end{array}$} & \multicolumn{2}{|c|}{$\begin{array}{c}\text { II } \\
\text { Faktor * Kovariate }\end{array}$} & \multicolumn{2}{|c|}{$\begin{array}{c}\text { III } \\
\text { Faktor im Modell }\end{array}$} \\
\hline & & $\mathbf{F}$ & $\mathbf{p}$ & $\mathbf{F}$ & $\mathbf{p}$ & $\mathbf{F}$ & $\mathbf{p}$ \\
\hline Alertness & $245 / 244$ & 4,769 & 0,029 & 64,415 & $<0,001^{*}$ & 9,711 & $0,002^{*}$ \\
\hline BZT & $236 / 225$ & 1,624 & 0,203 & 14,833 & $<0,001^{*}$ & 2,833 & 0,093 \\
\hline Dotting & $252 / 251$ & 1,348 & 0,246 & 49,807 & $<0,001^{*}$ & 3,647 & 0,057 \\
\hline LPS3 & $246 / 245$ & ,355 & 0,551 & 58,037 & $<0,001^{*}$ & 2,152 & 0,143 \\
\hline MWTB & $224 / 228$ & 1,470 & 0,026 & 12,493 & $<0,001^{*}$ & 1,013 & 0,315 \\
\hline Tapping & $251 / 250$ & 2,520 & 0,113 & 46,860 & $<0,001^{*}$ & 5,393 & 0,021 \\
\hline TMTa & $253 / 248$ & 1,624 & 0,203 & 47,486 & $<0,001^{*}$ & 4,418 & 0,036 \\
\hline TMTb & 237 / 231 & 0,512 & 0,475 & 46,799 & $<0,001^{*}$ & 2,386 & 0,123 \\
\hline VLMT & $220 / 222$ & 3,453 & 0,064 & 16,906 & $<0,001^{*}$ & 4,676 & 0,031 \\
\hline ZST & $248 / 250$ & 5,040 & 0,025 & 53,905 & $<0,001^{*}$ & 9,717 & $0,002^{*}$ \\
\hline Summenwert & $211 / 201$ & 2,784 & 0,096 & 42,802 & $<0,001^{*}$ & 5,687 & 0,018 \\
\hline
\end{tabular}

$\Delta \mathrm{CPÄ} / \mathrm{J} \downarrow$ bzw. $\uparrow:$ weniger bzw. mehr Steigerung der antipsychotischen Tagesdosis pro Jahr als Medianwert, BZT: Buchstaben-Zahlen-Test, LPS3: Leistungsprüfsystem Subtest 3, MWTB: Mehrfach-WortschatzIntelligenztest, p: Irrtumswahrscheinlichkeit, TMTa/b: Trail Making Test a/b, VLMT: Verbaler Lern- und Merkfähigkeitstest, ZST: Zahlen- Symbol-Test, *:Signifikanz unter Berücksichtigung der Bonferroni-Korrektur 


\subsubsection{Unterschiede in der Stichprobe der Frauen}

Aus Tabelle 14 ist ersichtlich, dass sich unter Berücksichtigung des Alters die beiden $\Delta \mathrm{CP} \ddot{\mathrm{J}} / \mathrm{J}$ Gruppen signifikant in fast allen neuropsychologischen Tests unterscheiden. Allein in der kristallinen Intelligenz (MWTB) zeigten sich keine signifikanten Unterschiede. Bis auf die Tests Alertness, LPS3 und MWTB erwiesen sich die Gruppenunterschiede auch nach Bonferroni-Korrektur als signifikant.

Tabelle 14: Kennwerte der ANOVA zur Prüfung der Assoziation des Alters mit den $\Delta \mathrm{CPÄ/J-}$ Gruppenunterschieden in neuropsychologischen Tests in der Stichprobe der Frauen

\begin{tabular}{|l|c|c|c|c|c|c|c|}
\hline \multirow{2}{*}{$\begin{array}{c}\text { neuropsychologischer } \\
\text { Test }\end{array}$} & $\boldsymbol{\Delta}$ CPÄ/J $\downarrow / \Delta$ CPÄ/J $\uparrow$ & \multicolumn{2}{c|}{$\begin{array}{c}\text { I } \\
\text { Faktor }\end{array}$} & \multicolumn{2}{c|}{$\begin{array}{c}\text { II } \\
\text { Faktor }{ }^{*} \text { Kovariate }\end{array}$} & \multicolumn{2}{c|}{ Faktor iIm Modell } \\
\cline { 3 - 8 } & & $\mathbf{F}$ & $\mathbf{p}$ & $\mathbf{F}$ & $\mathbf{p}$ & $\mathbf{F}$ & $\mathbf{p}$ \\
\hline Alertness & $115 / 177$ & 3,299 & 0,071 & 15,589 & $<0,001^{*}$ & 4,326 & 0,039 \\
\hline BZT & $111 / 113$ & 10,418 & $0,001^{*}$ & 12,365 & $<0,001^{*}$ & 12,389 & $<0,001^{*}$ \\
\hline Dotting & $112 / 118$ & 6,669 & 0,010 & 30,951 & $<0,001^{*}$ & 9,564 & $0,002^{*}$ \\
\hline LPS3 & $114 / 115$ & 5,274 & 0,023 & 31,837 & $<0,001^{*}$ & 7,987 & 0,005 \\
\hline MWTB & $108 / 113$ &, 292 & 0,590 & 1,631 & 0,198 &, 226 & 0,635 \\
\hline Tapping & $112 / 117$ & 8,344 & $0,004^{*}$ & 42,433 & $<0,001^{*}$ & 13,253 & $<0,001^{*}$ \\
\hline TMTa & $118 / 118$ & 7,815 & 0,006 & 46,940 & $<0,001^{*}$ & 12,307 & $<0,001^{*}$ \\
\hline TMTb & $112 / 110$ & 9,836 & $0,002^{*}$ & 43,570 & $<0,001^{*}$ & 15,644 & $<0,001^{*}$ \\
\hline VLMT & $105 / 110$ & 8,693 & $0,004^{*}$ & 16,611 & $<0,001^{*}$ & 10,794 & $0,001^{*}$ \\
\hline ZST & $116 / 118$ & 8,208 & 0,005 & 46,015 & $<0,001^{*}$ & 8,784 & $<0,001^{*}$ \\
\hline Summenwert & $97 / 99$ & 13,210 & $<0,001^{*}$ & 34,366 & $<0,001^{*}$ & 22,861 & $<0,001^{*}$ \\
\hline
\end{tabular}

$\triangle \mathrm{CPÄ} / \mathrm{J} \downarrow$ bzw. $\uparrow$ : weniger bzw. mehr Steigerung der antipsychotischen Tagesdosis pro Jahr als Medianwert, BZT: Buchstaben-Zahlen-Test, LPS3: Leistungsprüfsystem Subtest 3, MWTB: Mehrfach-WortschatzIntelligenztest, p: Irrtumswahrscheinlichkeit, TMTa/b: Trail Making Test a/b, VLMT: Verbaler Lern- und Merkfähigkeitstest, ZST: Zahlen- Symbol-Test, *:Signifikanz unter Berücksichtigung der Bonferroni-Korrektur

In den Stichproben beider Geschlechter konnte nachgewiesen werden, dass die Altersverteilung nicht die kognitiven Unterschiede zwischen den jeweils $2 \Delta \mathrm{CPÄ/J-Gruppen} \mathrm{bedingt.}$ Somit konnte die $\underline{\text { Hypothese } 4}$ auch unter Berücksichtigung des Alters angenommen werden.

\subsection{Betrachtung klinischer und medikamentöser Gruppenunter- schiede in den jeweiligen Subgruppen}

\subsubsection{Gruppenunterschiede in den Medikamentennebenwirkungen}

Da steigende und hohe Dosierungen der AP mit einem hohen Maß unterschiedlicher Nebenwirkungen assoziiert sind (s. Seite 8 f.), wurden die jeweils $2 \Delta \mathrm{CPÄ/J-Gruppen} \mathrm{diesbezüglich}$ verglichen. Hypothese 5 geht von einer höheren Rate an Nebenwirkungen in der jeweiligen $\triangle \mathrm{CP} \ddot{\mathrm{A}} / \mathrm{J}$-Gruppe aus. Zum einen wurden medikamentöse Nebenwirkungen, die sich in neurologischen Ausfallerscheinungen manifestieren, mit o.g. Inventaren erhoben und zwischen den Dosissteigerungsgruppen verglichen. Die Ergebnisse sind in Tabelle 15 dargestellt. Zum 
anderen sind die von den Probanden geäußerten Nebenwirkungssymptome in 4 Nebenwirkungskategorien zusammengefasst worden (vgl. Anhang D). Deskriptive und inferenzstatistische Kennwerte der $\Delta \mathrm{CPÄ/J-Gruppenvergleiche} \mathrm{sind} \mathrm{in} \mathrm{Tabelle} 16$ aufgeführt. So ist ersichtlich, dass sich bei beiden Geschlechtern hinsichtlich der subjektiv geäußerten Nebenwirkungen keine signifikanten Unterschiede zwischen den $\Delta \mathrm{CPÄ/J-Gruppen} \mathrm{ergaben.}$

Die neurologischen Nebenwirkungen unterschieden sich in der Stichprobe der Männer in den Bereichen der Akathisie (BAS) und tardiven Dyskinesie (TDRS) mit einer höheren Rate in der $\Delta \mathrm{CP} \ddot{\mathrm{A}} / \mathrm{J} \uparrow$-Gruppe signifikant. In der Stichprobe der Frauen zeigte sich die $\Delta \mathrm{CP} \ddot{\mathrm{A}} / \mathrm{J} \uparrow$-Gruppe in den Bereichen der dystonischen Rigidität ( $S A S)$ und der tardiven Dyskinesie (TDRS) als signifikant stärker beeinträchtigt.

Tabelle 15: Überprüfung der Gruppenunterschiede hinsichtlich neurologischer Nebenwirkungen in der Stichprobe der Männer und Frauen

\begin{tabular}{|c|c|c|c|c|c|c|c|c|c|}
\hline \multirow{2}{*}{$\begin{array}{l}\text { neurologische } \\
\text { Medikamenten- } \\
\text { nebenwirkungen }\end{array}$} & \multirow{2}{*}{$\begin{array}{l}\mathrm{A}=\Delta \mathrm{CPÄ} / \mathbf{J} \downarrow \\
\mathrm{B}=\Delta \mathrm{CPÄ} / \mathbf{J} \uparrow\end{array}$} & \multicolumn{4}{|c|}{ Männer } & \multicolumn{4}{|c|}{ Frauen } \\
\hline & & $\mathbf{n}$ & $\begin{array}{l}\text { Medi- } \\
\text { an }\end{array}$ & K-S-Z & $\mathbf{p}$ & $\mathbf{n}$ & $\begin{array}{l}\text { Medi- } \\
\text { an }\end{array}$ & K-S-Z & p \\
\hline \multirow{2}{*}{ AIMS } & A & 252 & 0,00 & \multirow{2}{*}{0,279} & \multirow{2}{*}{1,000} & 114 & 0,00 & \multirow{2}{*}{1,022} & \multirow{2}{*}{0,247} \\
\hline & $B$ & 250 & 0,00 & & & 116 & 0,00 & & \\
\hline \multirow{2}{*}{ BAS } & $A$ & 252 & 0,00 & \multirow{2}{*}{1,443} & \multirow{2}{*}{0,031} & 114 & 0,00 & \multirow{2}{*}{1,351} & \multirow{2}{*}{0,052} \\
\hline & $B$ & 251 & 0,00 & & & 116 & 2,00 & & \\
\hline \multirow{2}{*}{ SAS } & A & 252 & 1,00 & \multirow{2}{*}{1,274} & \multirow{2}{*}{0,078} & 114 & 0,00 & \multirow{2}{*}{2,037} & \multirow{2}{*}{$<0,001^{*}$} \\
\hline & $B$ & 251 & 1,00 & & & 116 & 0,00 & & \\
\hline \multirow{2}{*}{ TDRS } & A & 251 & 0,00 & \multirow{2}{*}{1,651} & \multirow{2}{*}{$0,008^{*}$} & 114 & 0,00 & \multirow{2}{*}{1,460} & \multirow{2}{*}{0,028} \\
\hline & $B$ & 251 & 1,00 & & & 116 & 1,00 & & \\
\hline
\end{tabular}

AIMS: Abnormal Involuntary Movement Scale, BAS: Barnes Akathisia Scale; K-S-Z: Kolmogorov Smirnov-Z Mdn: Median, p: Irrtumswahrscheinlichkeit, SAS: Simpson Angus Scale, TDRS, Tardive Dyskinesia Rating Scale; $\Delta \mathrm{CPÄ} / \mathrm{J} \downarrow$ bzw. $\uparrow:$ weniger bzw. mehr Steigerung der antipsychotischen Tagesdosis pro Jahr als Medianwert, *: Signifikanz unter Berücksichtigung der Bonferroni-Korrektur

Tabelle 16: Überprüfung der Gruppenunterschiede hinsichtlich von Probanden angegebener Nebenwirkungen in der Stichprobe der Männer und Frauen

\begin{tabular}{|c|c|c|c|c|c|c|c|}
\hline \multirow[b]{2}{*}{$\begin{array}{c}\text { Neben- } \\
\text { wirkungen }\end{array}$} & \multirow[b]{2}{*}{$\begin{array}{l}\mathrm{A}=\Delta \mathrm{CPÄ} / \mathbf{J} \downarrow \\
\mathrm{B}=\Delta \mathrm{CPÄ} / \mathbf{J} \uparrow\end{array}$} & \multicolumn{3}{|c|}{ Männer } & \multicolumn{3}{|c|}{ Frauen } \\
\hline & & n (\%) & $\mathbf{p}$ & K-S-Z & n (\%) & $\mathbf{p}$ & K-S-Z \\
\hline \multirow{2}{*}{$\begin{array}{l}\text { kardio- } \\
\text { vaskulär }\end{array}$} & A & $1(0,4)$ & \multirow{2}{*}{$1,000^{a)}$} & & $1(0,8)$ & \multirow{2}{*}{$1,000^{a)}$} & \\
\hline & $\mathrm{B}$ & $1(0,4)$ & & & $1(0,8)$ & & \\
\hline \multirow{2}{*}{$\begin{array}{l}\text { metabolisch- } \\
\text { endokrin }\end{array}$} & $A$ & $65(25,4)$ & \multirow{2}{*}{, $498^{a)}$} & & $29(24,2)$ & \multirow{2}{*}{$1,000^{a)}$} & \\
\hline & $B$ & $74(28,9)$ & & & $28(23,1)$ & & \\
\hline \multirow{2}{*}{ neurologisch } & $A$ & $27(10,5)$ & \multirow{2}{*}{, $091^{a)}$} & & $19(15,8)$ & \multirow{2}{*}{, $875^{\mathrm{a})}$} & \\
\hline & $\mathrm{B}$ & $42(16,4)$ & & & $21(17,4)$ & & \\
\hline \multirow{2}{*}{ psychiatrisch } & $A$ & $37(14,5)$ & \multirow{2}{*}{, $326^{a)}$} & & $12(10)$ & \multirow{2}{*}{, $215^{\mathrm{a})}$} & \\
\hline & $B$ & $47(18,4)$ & & & $20(16,5)$ & & \\
\hline gesamt & $A$ & $96(37,5)$ & $1,000^{b)}$ & ,289 & $50(41,67)$ & $0,994^{\text {b) }}$ & 0,425 \\
\hline
\end{tabular}

a) analysiert mittels Binomial-Test, b) analysiert mittels Kolmogorov-Smirnov-Test K-S-Z: Kolmogorov-SmirnovZ, p: Irrtumswahrscheinlichkeit, $\Delta \mathrm{CP} \ddot{\mathrm{A}} / \mathrm{J} \downarrow$ bzw. $\uparrow$ : weniger bzw. mehr Steigerung der antipsychotischen Tagesdosis pro Jahr als Medianwert 
Da auch nach Bonferroni-Korrektur signifikante Unterschiede zwischen den $\Delta \mathrm{CPÄ} / \mathrm{J}$ Gruppen bestanden, konnte die Hypothese 5 in Bezug auf die objektiven neurologischen Medikamentennebenwirkungen für beide Geschlechter angenommen werden. Im Hinblick auf die subjektiv geäußerten Nebenwirkungen musste Hypothese 5 jedoch verworfen werden.

\subsubsection{Gruppenunterschiede in der Komedikation}

Auf Seite $11 \mathrm{f}$. wurde darauf eingegangen, dass nicht nur die AP, sondern auch die Begleitmedikation modulatorisch auf das kognitive Funktionsniveau wirken kann. Deshalb wurde in beiden Geschlechtern getestet, inwieweit sich die beiden $\Delta \mathrm{CPÄ/J-Gruppen} \mathrm{in} \mathrm{der} \mathrm{Begleitme-}$ dikation unterscheiden. Tabelle 17 fasst die Ergebnisse zusammen.

Tabelle 17: Überprüfung der Gruppenunterschiede hinsichtlich der Komedikation in der Stichprobe der Männer und Frauen

\begin{tabular}{|c|c|c|c|c|c|}
\hline \multirow{2}{*}{ Komedikation } & \multirow{2}{*}{$\begin{array}{l}\mathrm{A}=\Delta \mathrm{CPÄ} / \mathbf{J} \downarrow \\
\mathrm{B}=\Delta \mathrm{CPÄ} / \mathbf{J} \uparrow\end{array}$} & \multicolumn{2}{|c|}{ Männer } & \multicolumn{2}{|c|}{ Frauen } \\
\hline & & n (\%) & $\mathbf{p}$ & n (\%) & $\mathbf{p}$ \\
\hline \multirow{2}{*}{$\begin{array}{c}\text { andere Antihyperki- } \\
\text { netika }\end{array}$} & $A$ & $2(0,8)$ & \multirow{2}{*}{0,289} & $2(1,7)$ & \multirow{2}{*}{1,000} \\
\hline & $B$ & $6(2,3)$ & & $2(1,7)$ & \\
\hline \multirow{2}{*}{ Anticholinergika } & $\mathrm{A}$ & $32(12,5)$ & \multirow{2}{*}{$0,005^{*}$} & $13(10,8)$ & \multirow{2}{*}{$0,028^{*}$} \\
\hline & $B$ & $60(23,4)$ & & $28(23,1)$ & \\
\hline \multirow{2}{*}{ Antidepressiva } & A & $52(20,3)$ & \multirow{2}{*}{$0,300^{a}$} & $32(26,7)$ & \multirow{2}{*}{$1,000^{\mathrm{a}}$} \\
\hline & $B$ & $41(16)$ & & $31(25,6)$ & \\
\hline \multirow{2}{*}{ Benzodiazepine } & $A$ & $22(8,6)$ & \multirow{2}{*}{0,023} & $17(14,2)$ & \multirow{2}{*}{$0,002^{*}$} \\
\hline & $\mathrm{B}$ & $41(16)$ & & $41(33,9)$ & \\
\hline \multirow{2}{*}{ Hypnotika } & $\mathrm{A}$ & $7(2,7)$ & \multirow{2}{*}{0,774} & $6(5)$ & \multirow{2}{*}{0,508} \\
\hline & $\mathrm{B}$ & $5(2,0)$ & & $3(2,5)$ & \\
\hline \multirow{2}{*}{$\begin{array}{l}\text { Phasenprophy- } \\
\text { laktika }\end{array}$} & A & $49(19,1)$ & \multirow{2}{*}{$0,530^{a}$} & $23(19,2)$ & \multirow{2}{*}{$1,000^{\circ}$} \\
\hline & $\mathrm{B}$ & $42(16,4)$ & & $24(19,8)$ & \\
\hline
\end{tabular}

p: Irrtumswahrscheinlichkeit, $\Delta \mathrm{CPÄ} / \mathrm{J} \downarrow$ bzw. $\uparrow:$ weniger bzw. mehr Steigerung der antipsychotischen Tagesdosis pro Jahr als Medianwert, *:Signifikanz nach Bonferroni-Korrektur

Hypothese 6 konnte angenommen werden, da Binomial-Tests einen nach BonferroniKorrektur signifikanten Unterschied zwischen den $\Delta \mathrm{CP} \ddot{\mathrm{A}} / \mathrm{J}$-Gruppen beider Geschlechter in der Applikation von Komedikation aufdeckten. Mehr Probanden der jeweiligen $\Delta \mathrm{CPÄ/J \uparrow -}$ Gruppe nahmen zusätzlich zu den AP Anticholinergika und/oder Benzodiazepine ein.

\subsubsection{Gruppenunterschiede in der Krankheitsschwere}

In der Bestrebung, Schizophrene von ihrem Leidensdruck zu entlasten und eine Verbesserung in psychopathologischer Symptomatik, kognitiver Leistung und alltäglichem Funktionsniveau zu erreichen, wird die antipsychotische Therapie in vielen Fällen auf sehr hohe Dosen gesteigert (Ito et al. 2005). Aus dieser Erkenntnis und der Tatsache, dass die bislang verfügbaren Antipsychotika nur eine begrenzte Wirkung auf die Erkrankungssymptomatik zeigen (Lieberman et al. 2005; Leucht et al. 2009 a), wurde Hypothese 7 formuliert. Diese nimmt an, dass Probanden mit einer größeren Erkrankungsschwere eine höhere Dosissteigerung pro Medika- 
tionsjahr erfahren als solche mit moderater Dosissteigerung. Deshalb wurden Gruppenunterschiede zwischen den Dosissteigerungsgruppen bezüglich Werten der klinischen Untersuchungsinventare CGI, GAF, PANSS und CNI statistisch geprüft. Tabelle 18 gibt einen Überblick hinsichtlich deskriptiver und inferenzstatistischer Kennwerte der Erkrankungsschwere der Dosissteigerungsgruppen in der Männer- und Frauenstichprobe.

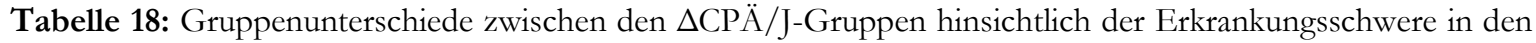
Stichproben der Männer und Frauen

\begin{tabular}{|c|c|c|c|c|c|c|c|c|c|}
\hline \multirow[b]{2}{*}{ Maße der Psychopathologie } & \multirow[b]{2}{*}{$\begin{array}{l}\mathbf{A}=\Delta \mathrm{CPÄ} / \mathbf{J} \downarrow \\
\mathbf{B}=\Delta \mathrm{CPÄ} / \mathbf{J} \uparrow\end{array}$} & \multicolumn{4}{|c|}{ Männer } & \multicolumn{4}{|c|}{ Frauen } \\
\hline & & $\mathbf{n}$ & Mdn & K-S-Z & $\mathbf{P}$ & $\mathbf{n}$ & Mdn & K-S-Z & $\mathbf{p}$ \\
\hline \multirow{2}{*}{ CGI } & A & 252 & 5,00 & \multirow{2}{*}{1,678} & \multirow{2}{*}{$0,007^{*}$} & 116 & 5,00 & \multirow{2}{*}{1,474} & \multirow{2}{*}{0,026} \\
\hline & $\mathrm{B}$ & 249 & 6,00 & & & 117 & 6,00 & & \\
\hline \multirow{2}{*}{ GAF } & A & 251 & 50,00 & \multirow{2}{*}{2,107} & \multirow{2}{*}{$<0,001^{*}$} & 115 & 51,00 & \multirow{2}{*}{1,813} & \multirow{2}{*}{$0,003^{*}$} \\
\hline & $\mathrm{B}$ & 249 & 40,00 & & & 116 & 37,50 & & \\
\hline \multirow{2}{*}{ PANSS allgemein } & A & 246 & 30,00 & \multirow{2}{*}{2,010} & \multirow{2}{*}{$<0,001^{*}$} & 114 & 28,50 & \multirow{2}{*}{2,002} & \multirow{2}{*}{$<0,001^{*}$} \\
\hline & $\mathrm{B}$ & 248 & 33,00 & & & 115 & 35,00 & & \\
\hline \multirow{2}{*}{ PANSS negativ } & A & 248 & 16,00 & \multirow{2}{*}{1,665} & \multirow{2}{*}{$0,008^{*}$} & 113 & 13,00 & \multirow{2}{*}{2,152} & \multirow{2}{*}{$<0,001^{\star}$} \\
\hline & $\mathrm{B}$ & 250 & 18,50 & & & 115 & 19,00 & & \\
\hline \multirow{2}{*}{ PANSS positiv } & A & 251 & 11,00 & \multirow{2}{*}{2,094} & \multirow{2}{*}{$<0,001^{*}$} & 114 & 10,50 & \multirow{2}{*}{1,722} & \multirow{2}{*}{$0,005^{*}$} \\
\hline & $\mathrm{B}$ & 248 & 13,00 & & & 114 & 13,50 & & \\
\hline \multirow{2}{*}{ CNI } & A & 234 & ,1364 & \multirow{2}{*}{1,278} & \multirow{2}{*}{0,076} & 104 & ,1212 & \multirow{2}{*}{2,189} & \multirow{2}{*}{$<0,001^{*}$} \\
\hline & $B$ & 235 & ,1602 & & & 107 & ,2172 & & \\
\hline
\end{tabular}

CGI: Clinical Global Impression Scale, CNI: Cambridge Neurological Inventory, GAF: Global Assessment of Functioning Scale, K-S-Z: Kolmogorov Smirnov-Z Mdn: Median, $\Delta$ CPÄ/J $\downarrow$ bzw. $\uparrow:$ weniger bzw. mehr Steigerung der antipsychotischen Tagesdosis pro Jahr als Medianwert, p: Irrtumswahrscheinlichkeit, *: Signifikanz unter Berücksichtigung der Bonferroni-Korrektur

Sowohl in der Männer- als auch in der Frauenstichprobe sind die Probanden der $\Delta \mathrm{CP} \ddot{A} / \mathrm{J} \uparrow$ Gruppe in allen psychopathologischen Variablen, dem alltäglichen Funktionsniveau sowie dem eingeschätzten Erkrankungsgrad schwerer betroffen, als diejenigen der $\Delta \mathrm{CP} \ddot{\mathrm{I}} / \mathrm{J}$ Gruppe. Die Ergebnisse erwiesen sich in den durchgeführten U-Tests als signifikant. Auch nach Bonferroni-Korrektur bestanden signifikante Unterschiede, sodass aufgrund größerer Erkrankungsschwere der jeweiligen $\Delta \mathrm{CP} \ddot{A} / \mathrm{J} \uparrow$-Gruppe Hypothese 7 angenommen werden konnte. 


\section{DISKUSSION}

Die vorliegende Arbeit hat das Ziel, die relative Verteilung der antipsychotischen Tagesdosis in einer umfassenden Stichprobe von an Schizophrenie erkrankten Personen zu beschreiben, sowie eine Assoziation von Steigerung der antipsychotischen Tagesdosis und kognitiven Leistungen zu beurteilen.

Folgende Hypothesen konnten bestätigt werden:

1. Männer waren zum Untersuchungszeitpunkt der GRAS-Studie auf eine höhere antipsychotische Tagesdosis eingestellt als Frauen.

3. Männer erfuhren über die gesamte Medikationsdauer bis zum GRASUntersuchungszeitpunkt eine größere Steigerung der antipsychotischen Tagesdosis als Frauen.

4. Probanden mit hoher Dosissteigerung pro Jahr erlangen in kognitiven Leistungstests schlechtere Ergebnisse als Probanden mit moderater Dosissteigerung.

5. Probanden mit hoher Dosissteigerung pro Jahr zeigen mehr Nebenwirkungen der antipsychotischen Therapie als Probanden mit moderater Dosissteigerung.

6. Probanden mit hoher Dosissteigerung pro Jahr sind zu einem größeren Anteil auf kognitionsmodulierende Komedikation eingestellt als Probanden mit moderater Dosissteigerung.

7. Probanden mit hoher Dosissteigerung pro Jahr zeichnen sich durch höhere Krankheitsschwere aus als Probanden mit moderater Dosissteigerung.

Folgende Hypothese musste verworfen werden:

2. Männer erhielten bereits zum Zeitpunkt der Erstmedikation eine höhere antipsychotische Tagesdosis als Frauen.

Die Testung der Hypothesen erfolgte an einer repräsentativen Stichprobe von maximal 1085 umfassend körperlich, neurologisch, psychiatrisch und neuropsychologisch untersuchten Schizophrenen verschiedener Krankheitsstatus und -verlaufstypen. 


\subsection{Geschlechtsunterschiede der antipsychotischen Dosis}

\subsubsection{GRAS-Untersuchungszeitpunkt}

In der vorliegenden Arbeit konnte an einer großen Stichprobe eine durchschnittlich höhere antipsychotische Tagesdosis bei Männern festgestellt werden als im Vergleich bei den Frauen. Somit konnte die Hypothese 1, welche von einer höheren antipsychotischen Tagesdosis bei Männern ausging, angenommen werden. Pinals et al. (1996) fanden in der von ihnen untersuchten Stichprobe nach Korrektur von Einflussvariablen keine Differenz der antipsychotischen Tagesdosis zwischen den Geschlechtern. Sie postulierten, dass bereits zuvor beschriebene Geschlechtsunterschiede in der antipsychotischen Tagesdosis aller Wahrscheinlichkeit nach durch die von ihnen berücksichtigten Geschlechtsunterschiede von Einflussgrößen wie z.B. der Dauer der unbehandelten Psychose vor Erstmedikation, Gesamtdauer antipsychotischer Medikation, Typus des applizierten AP und der Erkrankungsschwere begründet waren (Pinals et al. 1996). In der vorliegenden Arbeit kommt diese Argumentation jedoch nicht zum Tragen, da sich Männer und Frauen nicht in Hinblick auf diese Größen unterschieden (vgl. Tab. 6). Jedoch muss der geschlechtsspezifische Habitus als wichtiger Einflussfaktor der antipsychotischen Tagesdosis kritisch betrachtet werden. Denn Männer sind durchschnittlich 15\% schwerer und größer als Frauen, was ein insgesamt größeres Verteilungsvolumen für Pharmaka bei Männern bedeutet (Ogden et al. 2004). Da AP lipophil sind, um im ZNS wirken zu können (Aktories et al. 2009), verteilen sich diese jedoch nicht im ganzen Körper, sondern diffundieren vor allem in das Fettgewebe. Im Verhältnis zu Frauen ist jedoch der relative Anteil des Fettgewebes bei Männern um 10-15\% kleiner (Steinkamp et al. 1965). Diese Tatsache zeigt zum einen, dass das tatsächliche Verteilungsvolumen der AP nicht korrekt über die Körpergröße oder das Körpergewicht bestimmbar ist. Zum anderen wird deutlich, dass sich die tatsächlichen Verteilungsvolumina von AP zwischen den Geschlechtern nicht so wesentlich voneinander unterscheiden wie die körperliche Konstitution vermuten lässt. Aufgrund dessen wurden in der vorliegenden Arbeit die antipsychotischen Tagesdosisdifferenzen zwischen den Geschlechtern nicht für die Einflussgröße, Körpergröße und Körpergewicht korrigiert. So konnten auch Andia et al. (1995) zeigen, dass Frauen unabhängig vom Körpergewicht eine geringere antipsychotische Tagesdosis bekamen als Männer (Andia et al. 1995). Es erscheint unwahrscheinlich, dass evtl. unterschiedliche geschlechtsspezifische Verteilungsvolumina die gezeigten deutlichen antipsychotischen Tagesdosisunterschiede zwischen den Geschlechtern hinreichend erklären.

Eine Ursache der beschriebenen antipsychotischen Dosisdifferenzen zwischen Individuen wird in deren Rauchverhalten gesehen (Zevin und Benowitz 1999). Durch Enzyminduktion AP verstoffwechselnder Enzyme durch das Rauchen (z.B. CYP 1A2) sinkt der Plasmaspiegel 
einiger verabreichter AP (z.B. Haloperidol, Olanzapin, Risperidon) (Winterer 2010). Um vergleichbare Plasmaspiegel von AP zu erreichen, muss deshalb die antipsychotische Tagesdosis bei rauchenden Schizophrenen höher sein als bei nichtrauchenden. In der untersuchten Stichprobe war der Anteil an Rauchern bei Männern signifikant höher als bei Frauen. So kann gefolgert werden, dass die Differenz der antipsychotischen Tagesdosis zwischen den Geschlechtern durch die Unterschiede im Rauchverhalten mitbedingt wurde. Die Störgröße „Rauchen“ wurde in der vorliegenden Arbeit jedoch nicht berücksichtigt, da nicht bekannt ist, inwieweit die Induktion der Enzyme von der „Rauch-Tagesdosis“ abhängt und sich in der untersuchten Stichprobe dahingehend zwischen Männern und Frauen unterscheidet. Zudem ist nur von einigen AP (s.o.) eine Assoziation von Rauchen mit der Höhe des AP-Plasmaspiegels bekannt. Weiterhin wird davon ausgegangen, dass die durch Rauchen verursachte Enzym-Induktion stark vom Genotyp abhängt und somit bei einigen Rauchern den Serumspiegel der AP beeinflusst, bei anderen hingegen nicht (Ohara et al. 2003). Um letztlich einen definitiven Effekt des Rauchens auf die antipsychotische Tagesdosis zu erheben, müssten sowohl die antipsychotische Tagesdosis enzyminduktionsabhängiger AP, deren Plasmakonzentration, das exakte Rauchverhalten, der Genotyp des verstoffwechselnden Enzyms sowie deren gegenseitige Interaktion longitudinal beobachtet werden. Da die longitudinale Beobachtung dieser Parameter in $\operatorname{der} G R A S$-Untersuchung nicht gewährleistet werden konnte, kann lediglich vermutet werden, dass das Rauchverhalten die Geschlechtsunterschiede der antipsychotischen Tagesdosis mitbedingt.

Die insgesamt geringere antipsychotische Tagesdosis bei Frauen kann aber auch auf eine höhere Sensitivität gegenüber AP schließen lassen. Diesbezüglich wurde in der Vergangenheit häufig die Bedeutung des weiblichen Sexualhormons Östrogen diskutiert. Da es direkt in die Neurotransmitterinteraktion und -verstoffwechselung eingreift, äußert sich dessen Wirkung in einer Verbesserung der schizophrenen Krankheitssymptomatik (Taylor 1969; Bosse und DiPaolo 1996; Hafner 2003; Aichhorn et al. 2005). Sexualhormone bedingen jedoch auch psychosoziale Geschlechtsunterschiede, die letztlich zu höheren antipsychotischen Tagesdosen bei Männern führen könnten. So wird vermutet, dass durch eine mögliche protektive Wirkung von Östrogen die Schizophrenie bei Frauen später ausbricht. Deshalb können schizophrene Frauen durchschnittlich auf eine bessere schulische und berufliche Ausbildung sowie ein solideres soziales Netzwerk im Vergleich zu Männern zurückgreifen, was als möglich Ursache eines milderen Erkrankunsverlaufes in den ersten Erkrankungsjahren gesehen wird und somit eine geringere antipsychotische Dosierung notwendig werden lässt (Hafner 2003; Ito et al. 2005; Seeman MV 2009). Für die gefundenen Unterschiede der antipsychotischen Medikation kann in der vorliegenden Arbeit dieser Aspekt beigetragen haben. Frauen waren bei Ersterkrankung im Median 26 Jahre alt, Männer unterschieden sich hingegen mit 22 Jahren im Me- 
dian signifikant von ihnen. Die Bedeutung der sozialen Geschlechtsunterschiede auf die antipsychotische Tagesdosis könnte in Folgestudien untersucht werden. Da die Höhe des SerumÖstrogenspiegels mit der Psychopathologie assoziiert ist, führen Schwankungen im SerumÖstrogenspiegel zu schwankender Bedürftigkeit im Hinblick auf antipsychotische Therapie. Prä- und postmenopausale Frauen unterscheiden sich in der Höhe des Östrogens deutlich von gleichaltrigen Männern. So benötigen postmenopausale Frauen eine höhere antipsychotische Dosierung als prämenopausale (Salokangas 2004). Trotz des höheren Alters der Frauen zum GRAS-Untersuchungszeitpunkt, was auf einen deutlichen Anteil an postmenopausalen Frauen in der untersuchten Stichprobe schließen lässt (vgl. Anhang G), kann die Östrogenhypothese die Geschlechtsunterschiede der antipsychotischen Tagesdosis nicht hinreichend erklären. Inwiefern und weshalb sich Altersklassen bezüglich der antipsychotischen Tagesdosierung zwischen den Geschlechtern unterscheiden ist noch nicht vollständig geklärt. Bekannt ist jedoch, dass sich die Aktivität der AP verstoffwechselnden Enzyme mit einer geringeren Aktivität bei Frauen zwischen den Geschlechtern unterscheidet (Aichhorn et al. 2006; Aichhorn et al. 2007; Leon et al. 2007; Citrome et al. 2009). Diese Tatsache führt zu höheren Plasmaspiegeln von AP bei Frauen im Vergleich zu Männern, sodass Frauen bei gleicher antipsychotischer Dosierung höhere Plasmakonzentrationen der AP erreichen.

$\mathrm{Da}$ in der GRAS-Studie Plasmaspiegel von AP und Östrogen nicht gemessen wurden und keine Genanalysen verstoffwechselnder Enzyme durchgeführt wurden, konnten diese Größen in den Analysen der vorliegenden Arbeit nicht berücksichtigt werden. Geschlechtsunterschiede in der antipsychotischen Tagesdosis sind am ehesten als multifaktoriell und kumulativ anzusehen, da der ursächliche Geschlechtsdimorphismus auf unterschiedlichen Ebenen (hirnmorphologisch, funktional und psychosozial) zu unterschiedlichen Zeiten greift (Leung und Chue 2000). Die Annahme der Hypothese 1 lässt auf eine höhere Sensitivität der Frauen gegenüber AP schließen, deren Ursachen aber bislang noch nicht endgültig geklärt sind.

\subsubsection{Erstmedikation}

Entgegen der Annahme in Hypothese 2 wurden überraschenderweise keine geschlechtsspezifischen Unterschiede in der antipsychotischen Dosis zum Zeitpunkt der Erstmedikation nachgewiesen. Eine mögliche Erklärung dieser Tatsache kann darin gesehen werden, dass bei Neuerkrankten das Ansprechen auf AP noch nicht bekannt ist und die wirksame antipsychotische Tagesdosis anfänglich, von „Standard“-Dosen ausgehend, eintitriert wird. Eine Empfehlung für Anfangsdosen gibt z.B. PORT (The Schizophrenia Patient Outcomes Research Team). Aus der Evidenz 248 einzelner Studien empfiehlt PORT, dass die Erstmedikation Schizophrener im unteren Bereich des antipsychotischen Tagesdosisspektrums von 300 bis 500 CPÄ liegen sollte (Lehman et al. 2004). Relevant für die Erklärung der nicht signifikanten 
antipsychotischen Tagesdosisdifferenzen in der vorliegenden Arbeit ist, dass in diesen Empfehlungen nicht zwischen Männern und Frauen differenziert wird. Zum Zeitpunkt der Erstellung der vorliegenden Arbeit konnten keine evidenzbasierten Studien zu geschlechtspezifischer Erstmedikationsdosis gefunden werden. Antipsychotische Erstmedikationsdosen werden häufig auf der Basis klinischer Dosisfindungsstudien definiert, in denen Frauen aufgrund der Effekte zyklischer Hormonschwankungen auf die Symptomatik unterrepräsentiert sind (Chaves und Seeman 2006). So wird auch in der deutschen Behandlungsleitlinie Schirophrenie (Gaebel und Falkai 2005) nicht grundlegend zwischen der antipsychotischen Therapie von Männer und Frauen unterschieden, oder auf geschlechtsspezifische Unterschiede hinsichtlich der Sensitivität AP gegenüber aufmerksam gemacht. So liegen behandelnden Psychiatern keine evidenzbasierten geschlechtsspezifischen Dosierungsempfehlungen für AP vor. Diese Tatsache könnte erklären, weshalb in der untersuchten Stichprobe sowohl bei den Frauen als auch bei den Männern das zentrale Lagemaß der antipsychotischen Erstmedikationsdosis bei 300 CPÄ lag. Demzufolge war die Hypothese 2 zu verwerfen. Eine höhere Sensitivität gegenüber AP bei Frauen annehmend, ist retrospektiv nicht zu klären, ob bei Erstmedikation der Schizophrenie die Frauen im Geschlechtsvergleich zu hoch, oder die Männer zu gering mit AP dosiert worden sind. Frauen weisen im höheren Ersterkrankungsalter oft eine schwerere psychopathologische Beeinträchtigung auf als Männer mit jüngerem Erkrankungsalter (Seeman MV 1997). Das Ersterkrankungsalter betrachtend kann somit auch eine relativ schwerere Erkrankung bei Erstmedikation der Frauen zu einer relativ höheren antipsychotischen Tagesdosis geführt haben. Dieser Aspekt könnte die gleich hohen antipsychotischen Tagesdosen beider Geschlechter bei Ersterkrankung begründen. Um diese Frage letztlich beantworten zu können, sind Untersuchungen und Auswertungen der Krankheitsschwere zum Zeitpunkt der Erstmedikation erforderlich, die in der vorliegenden Arbeit wegen des Querschnittsdesigns der GRAS-Studie jedoch nicht zur Verfügung standen.

\subsection{Geschlechtsunterschiede der antipsychotischen Dosissteige- rung über die Medikationsdauer}

Die Auswertungen in der vorliegenden Stichprobe zeigen eine Zunahme der antipsychotischen Dosis vom Zeitpunkt der Erstmedikation bis zum Zeitpunkt der GRAS-Untersuchung in beiden Geschlechtern. Jedoch erwies sich die antipsychotische Dosissteigerung über die Medikationsdauer bei Männern als signifikant größer als bei Frauen. Somit konnte Hypothese 3, welche eine höhere antipsychotische Dosissteigerung bei Männern postulierte, angenommen werden. 
Zum Zeitpunkt der Erstellung der vorliegenden Arbeit lag keine vergleichbare Studie vor, die die Ursache antipsychotischer Dosissteigerung über die Medikationsdauer sowie etwaiger geschlechtsspezifischer Unterschiede untersucht hatte. Deshalb kann die Hypothese 3 nicht konkret mit bereits zu diesem Thema veröffentlichten Ergebnissen bestätigt, oder vor deren Hintergrund diskutiert werden. Auch können aufgrund des Querschnittstudiendesigns keine konkreten Gründe für die antipsychotische Dosissteigerung innerhalb der untersuchten Stichproben ausgelegt werden. Jedoch soll aufgeführt werden, inwieweit Geschlechtsunterschiede von Einflussgrößen des $\Delta \mathrm{CP} \ddot{\mathrm{A}} / \mathrm{J}$ die Geschlechtsunterschiede hinsichtlich der antipsychotischen Dosissteigerung bedingt haben könnten.

Wie bereits ausgeführt, rauchte ein großer Anteil der untersuchten Probanden zum Zeitpunkt der GRAS-Untersuchung Zigaretten. Auf Seite 16 f. wurde beschrieben, dass Rauchen über die Enzyminduktion AP verstoffwechselnder Enzyme die Wirksamkeit einiger AP mindern kann. Es ist bekannt, dass über den Erkrankungsverlauf hinweg und unter antipsychotischer Therapie die Rate an Rauchern zunimmt (Dalack et al. 1998). Somit könnte in der vorliegenden Untersuchung eine steigende Rate an Rauchern ab dem Zeitpunkt der Erstmedikation die durchschnittliche Steigerung der antipsychotischen Tagesdosis in den untersuchten Stichproben mitbegründet haben. Zum Zeitpunkt der Analysen lag keine Information über das Rauchverhalten der Probanden bei Erstmedikation vor. Dementsprechend konnte dieser Aspekt als Einflussgröße auf die Hypothese 3 nicht verifiziert werden. Hingegen können Änderungen im Medikationsverhalten der Psychiater innerhalb der letzten Jahre als wesentlicher Grund von antipsychotischer Dosissteigerung angenommen werden. Studien zeigen, dass der Anteil von „polypharmazierten“ (mehr als 1 AP zeitgleich) Schizophrenen zwischen 1999 und 2005 von 3,3\% auf 13,7\% gestiegen ist und sich dieser Trend verstärkt fortsetzt (Gilmer et al. 2007; Mojtabai und Olfson 2010). Auch in der untersuchten Stichprobe bekamen Probanden beider Geschlechter zum GRAS-Untersuchungszeitpunkt eine signifikant höhere Anzahl gleichzeitig verschriebener AP als zum Zeitpunkt der Erstmedikation. Polypharmazie sollte insgesamt kritisch betrachtet werden und die Ausnahme darstellen, da diese zu Arzneimittelinteraktionen, einem höheren $\mathrm{Ma} ß$ an Nebenwirkungen und extremen antipsychotischen Tagesdosen führt (Honer et al. 2006; Mojtabai und Olfson 2010). In den untersuchten Stichproben kann die beobachtete antipsychotische Dosissteigerung mit der über die Erkrankungsdauer steigenden Anzahl gleichzeitig verabreichter AP begründet werden, da in beiden Geschlechtern die beschriebene Medikationsdauer einen Medianwert von über 10 Jahren aufweist. Bei den Männern war die Rate der Patienten mit 2 und mehr AP pro Tag zum GRASUntersuchungszeitpunkt größer als bei Frauen. Es bleibt jedoch zu beachten, dass die Geschlechter sich nicht signifikant in der Summe der applizierten AP zum Zeitpunkt der Erstmedikation unterschieden. Somit kann davon ausgegangen werden, dass die höhere Steigerung 
zeitgleich verschriebener AP über den Medikationszeitraum bei Männern zu den Geschlechtsunterschieden in der antipsychotischen Dosissteigerung geführt hat. Retrospektiv ist schwer zu schließen, was zu der höheren Dosissteigerung bei Männern geführt hat. Ito et al. (2005) fanden heraus, dass ein persistierender (schlechter) Gesundheitszustand des Patienten, die skeptische Einstellung behandelnder Psychiater Verordnungsalgorithmen gegenüber und das Verlangen nach höheren Dosen seitens des betreuenden Pflegepersonals für sehr hohe antipsychotischen Dosen und Polypharmazie über die Erkrankungsdauer hinweg verantwortlich gemacht werden könnte (Ito et al. 2005). In der vorliegenden Untersuchung können diese Aspekte und auch eine Zunahme der Krankheitsschwere über die Medikationsdauer zu der generellen Steigerung der antipsychotischen Tagesdosis geführt haben. Xiang et al. fanden in der von ihnen untersuchten Stichprobe eine Assoziation von zunehmender Polypharmazie und Krankheitsschwere (Xiang et al. 2007). Auf der Basis der vorliegenden Ergebnisse ist der Grund der geschlechtsspezifischen antipsychotischen Dosissteigerung nicht zu benennen, da die Untersuchungen in einem Querschnittsdesign durchgeführt wurden. Informationen über die Geschlechtsdifferenzen der Erkrankungsschwere bei Ersterkrankung lagen für die statistische Auswertung nicht vor. Männer und Frauen unterschieden sich zum GRASUntersuchungszeitpunkt nicht in den Parametern der Erkrankungsschwere (vgl. Tab. 6). Somit kann nicht geklärt werden, ob die größere Steigerung der antipsychotischen Tagesdosis über die Medikationsdauer in der Stichprobe der Männer zu einer Verbesserung der Krankheitssymptomatik geführt hat und schließlich etwaige Geschlechtsdifferenzen in der Krankheitsschwere begründet oder nivelliert hat. Wie auf Seite $8 \mathrm{f}$. beschrieben, konnten bislang jedoch keine durchschlagenden medikamentösen Therapien etabliert werden, die zu einer deutlichen Verbesserung der Schizophrenie führen, sodass auch in der vorliegenden Arbeit eine Verbesserung der Krankheitsschwere durch antipsychotische Dosissteigerung nicht angenommen werden kann.

Als weitere Ursache antipsychotischer Dosissteigerung über die Medikationsdauer wird die abnehmenden Sensitivität gegenüber AP diskutiert (Seeman P et al. 2005; Samaha et al. 2007). Unter chronischer antipsychotischer Therapie reagiert das ZNS kompensatorisch mit der Expression supersensitiver $\mathrm{D}_{2}-\mathrm{R}$. Um diese wiederum zu blockieren, müssen höhere Dosen an AP verabreicht werden, um einer Verschlechterung der Erkrankung und Erkrankungsrückfällen vorzubeugen (Kirkpatrick et al. 1992). Da in der vorliegenden Stichprobe keine Untersuchung bezüglich der Dopaminsensitivität durchgeführt wurde, kann diese Ursache für die Dosissteigerung für beide Geschlechter lediglich vermutet werden. Aus folgendem Grund scheint es jedoch unwahrscheinlich, dass die Theorie der Supersensitivität der $\mathrm{D}_{2}-\mathrm{R}$ die Geschlechtsunterschiede und somit Hypothese 3 ursächlich begründet: Da die Frauen der untersuchten Stichprobe signifikant länger AP einnahmen als die Männer, müssten Frauen, die Su- 
persensitivitätstheorie annehmend, eine höhere Dosissteigerung pro Jahr aufweisen. In der untersuchten Stichprobe zeigten jedoch die Männer eine signifikant höhere antipsychotische Dosissteigerung pro Medikationsjahr. Aus bisher veröffentlichen Studien zu der $\mathrm{D}_{2}-\mathrm{R}-$ Supersensitivität geht nicht hervor, bei welchen Individuen in welcher Intensität ab welchem Zeitpunkt die Supersensitivität der $\mathrm{D}_{2}-\mathrm{R}$ einsetzt und ob sich diese Aspekte zwischen den Geschlechtern unterscheiden. Deshalb ist schwer zu beurteilen, inwieweit der antipsychotisch induzierte Effekt der Supersensititvität über die Erkrankungsdauer in den untersuchten Stichproben zu der beobachteten Dosissteigerung geführt hat und damit die Annahme der Hypothese 3 ursächlich begründen kann.

Letztlich kann die antipsychotische Dosisänderung über den Medikationsverlauf auch im „natürlichen“"Verlauf der Schizophrenie begründet liegen (vgl. Seite 12 f.). Uchida et al. (2008) verwiesen auf Schwankungen der antipsychotischen Dosis über die Medikationsdauer. So stellten sie in einer Untersuchung fest, dass die antipsychotische Dosis über die Lebenszeit einer umgekehrten U-Form gleicht. Demnach bekamen Patienten bis zum mittleren Lebensalter steigende antipsychotische Tagesdosen, welche in den darauffolgenden Jahren stabil blieben. Im hohen Lebensalter der Betroffenen sank die Höhe der antipsychotischen Tagesdosis wieder ab (Uchida et al. 2008). In der untersuchten Stichprobe war der Anteil an Probanden über dem 40. Lebensjahr zum Zeitpunkt der GRAS-Untersuchung in der Stichprobe der Frauen deutlich höher. Spiegelbildlich war der Anteil junger Probanden in der Stichprobe der Männer größer (vgl. Anhang G). So darf vermutet werden, dass in der untersuchten Stichprobe ein größerer Anteil der Frauen sich bereits in der Phase abnehmender Dosierung befand, wohingegen bei den Männern der Anteil jüngerer Probanden mit noch steigender Dosis überwog. Auch lagen die Werte der durchschnittliche Erkrankungsdauer und des Ersterkrankungsalters der untersuchten Männer deutlich unter denen der untersuchten Frauen (vgl. Tab. 6). Liebermann et al. (2001) verweisen darauf, dass zwischen 5 und 10 Jahren nach Ersterkrankung der kognitiv-degenerative Prozess der Schizophrenie am massivsten ist, worauf sich das Funktionsniveau der Betroffenen in den Folgejahren auf einem insgesamt niedrigeren Level stabilisiert (Lieberman et al. 2001). Diese Annahme kann als Grund der Dosisveränderungen über die Medikationsdauer gesehen werden, da Psychiater den starken Schwankungen innerhalb der ersten Erkrankungsjahre in Psychopathologie, Defiziten im alltäglichen Leben und hohen Rückfallraten häufig mit Dosissteigerungen der AP begegnen (Uchida und Mamo 2009). Da der relative Anteil an jüngeren und kürzer erkrankten Probanden in der Stichprobe der Männer größer war, kann von einer insgesamt „schwankenderen“ Psychopathologie der Männer ausgegangen werden, was schließlich eine höhere antipsychotische Dosissteigerung bei den Männern bewirkt haben kann. 
Inwieweit die „natürlich“ bedingten Geschlechtsunterschiede der Variablen Alter, Ersterkrankungsalter, Erkrankungsdauer und Erkrankungsschwere über den Krankheitsverlauf die Annahme der Hypothese 3 ursächlich mitbegründen, konnte letztlich nicht ausreichend belegt und interpretiert werden. Dieser Frage könnten Folgestudien nachgehen.

Den hier vorgelegten Ergebnissen der höheren antipsychotischen Dosissteigerung bei Männern stehen jedoch physiologische Geschlechtsunterschiede konträr gegenüber. Bei abnehmendem Serum-Östrogenspiegel im weiblichen Klimakterium verschlechtert sich die Symptomatik der Schizophrenie (Riecher-Rossler et al. 1994; Kulkarni et al. 2008). Deshalb wird bei Frauen eine höhere antipsychotische Tagesdosis zwischen dem 45. bis 55. Lebensjahr notwendig (Seeman MV 1996). Demnach müssten Frauen mit Anfang des Klimakteriums eine höhere Dosissteigerung erfahren als Männer, da sich bei Männern dieses Alters kein Ereignis mit einem so konkreten Effekt auf den Krankheitsverlauf ereignet, welches höhere antipsychotischen Dosen notwendig werden lässt. So ist postmenopausal der weibliche PlasmaÖstrogenspiegel niedriger als bei gleich alten Männern (Vermeulen et al. 2002). Die Östrogenhypothese annehmend, müsste diese Tatsache eine höhere antipsychotische Dosissteigerung bei postmenopausalen Frauen bedingen. Zudem kommt es im höheren Lebensalter bei Frauen in einem signifikant größeren Maße zur Reduktion des cerebralen Blutflusses als bei Männern (Gur und Gur 1990). Das führt im Vergleich zu Männern zu einer schlechteren Anflutung der AP bei Frauen. Darüber hinaus nimmt auch die Zahl zentraler $\mathrm{D}_{2}-\mathrm{R}$ bei Frauen langsamer ab als bei Männern, was ebenfalls zu einer geringeren Wirksamkeit der AP bei älteren Frauen im Vergleich zu gleich alten Männern führt (Pohjalainen et al. 1998). Somit sprechen die Östrogenhypothese, der Geschlechtsunterschied in $\mathrm{D}_{2}$-R-Expression und cerebralem Blutfluss eigentlich für eine höhere antipsychotische Dosissteigerung bei älteren Frauen als bei Männern, was den vorliegenden Ergebnissen und somit Hypothese 3 konträr gegenüber steht. In folgenden Studien kann getestet werden, ob Hypothese 3 auch unter Berücksichtigung verschiedener Altersklassen und aufgeführter biologischer Einflussfaktoren Bestand hat.

Schließlich kann die Geschlechtsdifferenz in der Dosissteigerung zwischen den Geschlechtern durch die berechnete Variable $\Delta \mathrm{CP} \ddot{A} / \mathrm{J}$ bedingt sein. Der Quotient $\Delta \mathrm{CP} \ddot{\mathrm{A}} / \mathrm{J}$ trägt die Medikationsdauer als Divisor. Durch eine längere Medikationsdauer bei Frauen wird deren Quotient relativ zu dem der Männer kleiner. Da alle Variablen in einer Querschnittsstudie erhoben wurden und lediglich Ergebnisse zweier Dosierungszeitpunkte vorlagen, konnte das Problem der Dosierungsänderung über das Alter nicht berücksichtigt werden. 


\subsection{Assoziation antipsychotischer Dosissteigerung mit der Kogni- tion}

In den vorangegangenen Kapiteln wurde verdeutlicht, dass Männer und Frauen im Hinblick auf das kognitive Profil, sowie das Reaktionsverhalten auf AP zwei sehr heterogene Gruppen darstellen (Goldstein et al. 1998; Seeman MV 2004; Rubin et al. 2008). Auf Grund dessen wurden die beiden Geschlechter in der Untersuchung von Assoziation antipsychotischer Dosissteigerung mit der Kognition parallel betrachtet. In beiden Geschlechtern wurde die $\Delta \mathrm{CP} \ddot{\mathrm{A}} / \mathrm{J} \uparrow$-Gruppe gegen die $\Delta \mathrm{CP} \ddot{\mathrm{A}} / \mathrm{J} \downarrow$-Gruppe hinsichtlich kognitiver Leistungen getestet (vgl. Seite 30 f.).

Die Ergebnisse zeigen, dass Probanden der $\Delta \mathrm{CP} \ddot{A} / \mathrm{J} \uparrow$-Gruppe in beiden Geschlechtern im Durchschnitt schlechtere kognitive Leistungen erzielten als diejenigen der $\Delta \mathrm{CPÄ/J \downarrow -Gruppe.}$ Somit konnte die Hypothese 4 angenommen werden. In der Männerstichprobe stellten sich signifikante Unterschiede der $\Delta \mathrm{CP} \ddot{\mathrm{A}} / \mathrm{J}$-Gruppe in den Bereichen Wahrnehmung und Aufmerksamkeit (Alertness / ZST) sowie psychomotorische Geschwindigkeit (ZST) heraus. Die Unterschiede dieser beiden Gruppen in der Stichprobe der Frauen erreichten Signifikanz in den Kategorien Wahrnehmung und Aufmerksamkeit (Alertness), Arbeitsgedächtnis (BZT), visuell motorische Geschwindigkeit und Feinmotorik (Dotting und Tapping), schlussfolgerndes Denken (LPS3), Informationsverarbeitungsgeschwindigkeit (TMTa), kognitive Flexibilität (TMTb), Lernen (VLMT), psychomotorische Geschwindigkeit (ZST) sowie dem neuropsychologischen Summenwert (Summenwert). Die Ergebnisse der vorliegenden Arbeit stehen dem Großteil klinischer Studien gegenüber, welche von einer uneingeschränkt kognitionsfördernden Wirkung, besonders der SGA, ausgehen.

Auf der Basis sorgfältiger Literaturrecherchen war es nicht möglich, veröffentlichte Studien zu finden, die den direkten Effekt der antipsychotischen Dosissteigerung auf Kognition untersuchten. Jedoch konnte in einer Studie eine Assoziation von höherer antipsychotischer Tagesdosis mit abnehmendem kognitiven Leistungsniveau festgestellt werden. So bekamen Probanden nach dem 37. Lebensjahr eine signifikant höhere antipsychotische Tagesdosis im Vergleich zu jüngeren Probanden; und die Gruppe der Älteren erreichte in neuropsychologischen Tests signifikant schlechtere Ergebnisse (Elie et al. 2010). Elie et al. (2010) stellten in dieser Studie ebenfalls heraus, dass hohe antipsychotische Dosen trotz Korrektur für das Alter und der psychopathologischen Erkrankungsschwere einen signifikant negativen Effekt auf den neuropsychologischen Summenwert zeigten. Wie in der vorliegenden Arbeit, bestand deren neuropsychologischer Summenwert aus Tests der kognitiven Domänen von Wortgedächtnis, Arbeitsgedächtnis, motorischer Geschwindigkeit, Wortflüssigkeit, schlussfolgerndem Denken und Informationsverarbeitungsgeschwindigkeit (Keefe et al. 2004). Auch in der CATIE-Studie 
wurde eine Assoziation schlechter kognitiver Leistungen mit höherer antipsychotischer Tagesdosis aufgezeigt, jedoch ohne zu folgern welches der kausale Faktor ist (Chakos et al. 2006).

Als Einflussfaktor auf die kognitiven Leistungen wurde vielfach der AP-Typus (SGA oder FGA) diskutiert, wobei man letztlich von einer gleichen Wirksamkeit auf die Krankheitssyptome ausgeht (Swartz et al. 2008; Davidson et al. 2009; Leucht et al. 2009 a/b). In der vorliegenden Studie war die Zugehörigkeit zu einer der beiden $\Delta \mathrm{CPÄ/J-Gruppen} \mathrm{in} \mathrm{beiden} \mathrm{Ge-}$ schlechtern signifikant mit dem Typus des AP zu GRAS-Untersuchungszeitpunkt assoziiert. Während sich in beiden Geschlechtern die jeweils $2 \Delta \mathrm{CPÄ/J-Gruppen} \mathrm{nur} \mathrm{gering} \mathrm{in} \mathrm{der} \mathrm{Ap-}$ plikationsrate von ausschließlich SGA unterschieden, bekamen die $\Delta$ CP $\ddot{A} / J \downarrow-G r u p p e n ~ z u$ einem größeren Anteil ausschließlich FGA. Allerdings war der Anteil der gemischten Applikation von FGA und SGA in der $\Delta \mathrm{CP} \ddot{\mathrm{A}} / \mathrm{J} \uparrow$-Gruppe in beiden Geschlechtern im Vergleich zu der $\Delta \mathrm{CP} \ddot{\mathrm{A}} / \mathrm{J} \downarrow$-Gruppe mehr als doppelt so hoch. Somit war schließlich der Gesamtanteil der Probanden, die zum GRAS-Untersuchungszeitpunkt mit FGA behandelt wurden, in den $\Delta \mathrm{CP} \ddot{\mathrm{A}} / \mathrm{J} \uparrow$-Gruppen höher als in den $\Delta \mathrm{CP} \ddot{\mathrm{A}} / \mathrm{J} \downarrow-G r u p p e n\left({ }^{\AA}: 35,6 \%\right.$ vs. 58,2\%; $+: 32,5$ vs. 47,9\%). Trotz der Annahme einer gleichen Wirksamkeit beider AP-Typen auf die Kognition (Keefe et al. 2007), ist diese Tatsache insofern wichtig, als FGA häufiger motorische Nebenwirkungen auslösen als SGA. Da die angewandten neuropsychologischen Tests LPS3, Dotting, Tapping, TMTa, TMTb, ZST und Alertness verschiedene Aspekte kognitiver Leistung über motorische Äußerungen (Hand- oder Fingerbewegungen) in Relation zur Zeit erfassen, können Nebenwirkungen der antipsychotischen Therapie zu schlechteren Ergebnissen dieser Tests geführt haben. In beiden Geschlechtern zeigte die $\Delta \mathrm{CPÄ/J \uparrow -Gruppe} \mathrm{signifikant} \mathrm{mehr} \mathrm{tardive}$ Dyskinesien. Darüber hinaus konnten in der $\Delta \mathrm{CPÄ/J \uparrow -Gruppe} \mathrm{der} \mathrm{weiblichen} \mathrm{Teilstichprobe}$ mehr EPMS dokumentiert werden. Bei den bereits genannten durchgeführten Leistungstests kann nicht gänzlich ausgeschlossen werden, dass Medikamentennebenwirkungen somit die $\triangle \mathrm{CP} \ddot{\mathrm{A}} / \mathrm{J}$-Gruppenunterschied der kognitiven Leistungen mitbegründen. Es ist jedoch zu berücksichtigen, dass Probanden der $\Delta \mathrm{CP} \ddot{\mathrm{A}} / \mathrm{J} \uparrow$-Gruppe in beiden Geschlechtern auch in von der Motorik unabhängigen kognitiven Leistungstest BZT und VLMT schlechtere Ergebnisse erzielten als Probanden der $\Delta \mathrm{CP} \ddot{A} / \mathrm{J} \downarrow$-Gruppe. In den Analysen von Elie et al. (2010) war ein geringeres kognitives Leistungsniveau mit hoher antipsychotischer Dosierung assoziiert, was nicht durch den Effekt etwaiger motorischer Nebenwirkungen der FGA erklärt werden konnte (Elie et al. 2010). So wird davon ausgegangen, dass die in der vorliegenden Arbeit gefundenen Unterschiede kognitiver Leistung zwischen den Dosissteigerungsgruppen auch nicht wesentlich durch den AP-Typus und motorische Nebenwirkungen begründet sind.

Wie bereits erwähnt wird Rauchen bei schizophrenen Patienten als kompensatorische Selbstmedikation kognitiver Defizite verstanden. Durch die Interaktion an z.B. zentralen Nikotin- 
a7-Rezeptoren kann Nikotin signifikant Lernen, Aufmerksamkeit und Gedächtnis verbessern (Levin et al. 1996; Rezvani und Levin 2001). In der Gesamtstichprobe rauchten 69,4\% aller Probanden. In der $\Delta \mathrm{CP} \ddot{\mathrm{A}} / \mathrm{J} \uparrow$-Gruppe rauchten 75,8\% der Männer und 59,5\% der Frauen im Vergleich zu der $\Delta \mathrm{CPÄ/J \downarrow -Gruppe,} \mathrm{in} \mathrm{der} \mathrm{77,3 \%} \mathrm{der} \mathrm{Männer} \mathrm{und} \mathrm{54,2 \%} \mathrm{der} \mathrm{Frauen} \mathrm{rauch-}$ ten. Die Differenzen zwischen den $\Delta \mathrm{CPÄ/J-Gruppen} \mathrm{in} \mathrm{beiden} \mathrm{Geschlechtern} \mathrm{hinsichtlich}$ der Anzahl Rauchender waren jedoch nicht signifikant. Somit kann das Rauchverhalten zwar einen Effekt auf die Ergebnisse kognitiver Testungen in den untersuchten Stichproben gehabt haben, jedoch können die $\Delta \mathrm{CPÄ/J-Gruppenunterschiede} \mathrm{in} \mathrm{den} \mathrm{kognitiven} \mathrm{Testungen} \mathrm{wegen}$ der vergleichbar hohen Raucherraten der Vergleichsgruppen nicht durch das Rauchverhalten bedingt sein.

Als wesentlich ist jedoch auch bei der Interpretation dieser Ergebnisse der „natürliche Verlauf" der Schizophrenie anzusehen. Dieser bedingt sowohl Unterschiede in der antipsychotischen Tagesdosis, als auch im kognitiven Leistungsniveau zwischen Individuen. So wird die Dauer der unbehandelten Psychose vor Erstmedikation (DUP) als eine Ursache schwerwiegender kognitiver Störungen im Krankheitsverlauf gedeutet und als Spiegel einer größeren Krankheitsschwere angesehen (Wyatt 1991; Perkins et al. 2005; Owens et al. 2010). Sowohl bei den Männern als auch bei den Frauen zeigten sich in der vorliegenden Untersuchung zwar Unterschiede in der DUP, mit jeweils längerer Dauer in den $\Delta \mathrm{CP} \ddot{A} / \mathrm{J} \uparrow-\mathrm{Gruppe}$, jedoch erreichten diese Differenzen keine Signifikanz. Demnach kann die DUP nicht für die kognitiven Differenzen zwischen den jeweils $2 \Delta \mathrm{CPÄ/J-Gruppen} \mathrm{verantwortlich} \mathrm{gemacht} \mathrm{werden.} \mathrm{Als}$ bedeutenderer Einflussfaktor des kognitiven Funktionsniveaus ist die Erkrankungsdauer zu interpretieren, legt man einen neurodegenerativen Prozess der Schizophrenie zugrunde. Jedoch unterschieden sich die $\Delta \mathrm{CPÄ/J-Gruppen} \mathrm{in} \mathrm{beiden} \mathrm{Geschlechtern} \mathrm{auch} \mathrm{in} \mathrm{dieser} \mathrm{Ein-}$ flussgröße nicht signifikant, sodass damit die kognitiven Unterschiede zwischen den jeweils 2 $\Delta \mathrm{CP} \ddot{A} / \mathrm{J}$-Gruppen nicht ursächlich begründet werden können.

In beiden Geschlechtern unterschieden sich die beiden $\Delta \mathrm{CPÄ/J-Gruppen} \mathrm{bezüglich} \mathrm{des} \mathrm{Erst-}$ erkrankungsalters, bei den Männern erreicht dieser Unterschied sogar Signifikanz. Eine frühe Unterbrechung der kognitiven und sozialen Reifung führt zu einem insgesamt geringeren kognitiven Funktionsniveau, da sowohl Wissen bis zu diesem Zeitpunkt, als auch Mechanismen des Lernens in geringerem Maße erworben werden können (Häfner 2004; Reichenberg et al. 2010). So ist bekannt, dass ein im Vergleich jüngeres Ersterkrankungsalter bei Schizophrenen mit größeren kognitiven Defiziten in den Bereichen psychomotorische Geschwindigkeit, kognitive Flexibilität, Aufmerksamkeit, Informationsverarbeitungsgeschwindigkeit und Arbeitsgedächtnis assoziiert ist (Rajji et al. 2009). Der signifikante Unterschied zwischen den Dosissteigerungsgruppen in der Stichprobe der Männer hinsichtlich des Alters bei Ersterkrankung kann folglich für die Leistungsunterschiede in den Tests Alertness und ZST mitverant- 
wortlich gewesen sein. Für die Ergebnisse in der Stichprobe der Frauen sowie Leistungsunterschiede weiterer kognitiver Tests zwischen den Dosissteigerungsgruppen liefert die Betrachtung des Ersterkrankungsalters jedoch keine zufriedenstellende Erklärung. Von größerer Bedeutung für die Interpretation kognitiver Gruppenunterschiede ist die Berücksichtigung des Alters zum GRAS-Untersuchungszeitpunkt. Ein höheres Lebensalter ist sowohl in schizophrenen, als auch gesunden Probanden mit der Abnahme kognitiver Leistungen in den Bereichen Gedächtnis, schlussfolgerndes Denken, Exekutivfunktionen und Wortschatz assoziiert (Kern et al. 2008; Harvey et al. 2010; Salthouse 2010). Dieser Aspekt gewinnt an Bedeutung, legt man einen neurodegenerativen Prozess der Schizophrenie zugrunde. In, den Analysen vorausgehenden, Berechnungen zeigte das Alter als einzige Variable eine konsistente Assoziation mit allen getesteten kognitiven Domänen. Deshalb wurde die Kovariate „Alter“ in die Analysen der vorliegenden Arbeit einbezogen. Die Assoziation des Alters mit dem kognitiven Leistungsniveau wurde im „Modell“ Alter* $\Delta C P A ̈ / J$ getestet. Die Ergebnisse zeigten bei beiden Geschlechtern höchst signifikante Unterschiede zwischen den beiden $\Delta \mathrm{CPÄ/J-Gruppen}$ in allen getesteten kognitiven Variablen, abgesehen vom prämorbiden Leistungsniveau bei Frauen. Dies war überraschend, da sich bei beiden Geschlechtern die $\Delta \mathrm{CPÄ/J-Gruppen} \mathrm{nicht}$ signifikant im Alter unterschieden. Die deutliche Assoziation der Variable Alter zum GRASUntersuchungszeitpunkt mit den kognitiven $\Delta \mathrm{CPÄ/J-Gruppenunterschieden} \mathrm{kann} \mathrm{jedoch}$ damit erklärt werden, dass das Alter stark mit den Variablen Erkerankungsdauer, Alter bei Ersterkrankung und Gesamtdauer der psychiatrischen Aufenthalte assoziiert war. Da diese Parameter Messgrößen der Krankheitsschwere darstellen, kann die $\Delta \mathrm{CP} \ddot{\mathrm{A}} / \mathrm{J} \uparrow-\mathrm{Gruppe}$ als schwerer erkrankt angesehen werden als die $\Delta \mathrm{CPÄ/J \downarrow -Gruppe.} \mathrm{In} \mathrm{großen} \mathrm{Studien} \mathrm{konnte} \mathrm{gezeigt} \mathrm{werden,} \mathrm{dass}$ junge Probanden, die später Schizophrenie entwickelten, zum einen Entwicklungsdefizite in den Bereichen verbaler und visueller Wissenserwerb, schlussfolgerndes Denken und Konzeptualisierung aufwiesen, zum andern Entwicklungsverzögerungen bei der Testung von Informationsverarbeitungsgeschwindigkeit, Aufmerksamkeit, Arbeitsgedächtnis und Problemlösungsverhalten zeigten (Häfner 2004; Reichenberg et al. 2010). Somit können die deutlichen kognitiven Unterschiede zwischen den $\Delta \mathrm{CP} \ddot{\mathrm{A}} / \mathrm{J}$-Gruppen in dem „Modell“ Alter* $\Delta \mathrm{CP} \ddot{\mathrm{A}} / \mathrm{J}$ durch Gruppenunterschiede im Ersterkrankungsalter interpretiert werden. Auffallend ist die Tatsache, dass sich die beiden $\triangle \mathrm{CPÄ/J-Gruppen} \mathrm{in} \mathrm{der} \mathrm{Männerstichprobe} \mathrm{im} \mathrm{direkten} \mathrm{Ver-}$ gleich der kristallinen/prämorbiden Intelligenz nicht signifikant unterschieden, jedoch ein hochsignifikanter Gruppenunterschied unter Berücksichtigung des Alters entstand. Der MWTB gilt definitionsgemäß als robust gegen die Störgröße „Alter“ (Lehrl 1999). So muss davon ausgegangen werden, dass bei den jüngeren und signifikant früher männlichen Erkrankten der $\triangle \mathrm{CP} \ddot{\mathrm{A}} / \mathrm{J} \uparrow$-Gruppe die abgefragten Wörter des MWTB nicht im Wortschatz vorhanden waren. Entweder war die Schizophrenie in $\operatorname{der} \Delta \mathrm{CP} \ddot{\mathrm{A}} / \mathrm{J} \uparrow-$ Gruppe vor Erwerb dieser 
Vokabeln ausgebrochen, oder die Vokabeln des Tests sind nicht mehr zeitgemäß und werden von im Vergleich jüngeren Personen nicht (mehr) erworben. Die gefundenen Differenzen zum GRAS-Untersuchungszeitpunkt könnten somit durch das Erkrankungsalter und damit bereits vor Erkrankungsbeginn bestandene Unterschiede in der Kognition, im Sinne von Unterschieden in erworbenen Kenntnissen und Fertigkeiten, mitbegründet sein. Um den Alterseffekt auf den MWTB berücksichtigen zu können, wären Vergleichsstudien besonders an jungen, gesunden Probanden notwendig. Es kann nämlich nicht ausgeschlossen werden, dass der gefundene Effekt ausschließlich durch den Erkrankungsprozess der Schizophrenie bedingt ist. Ebenfalls ist vorstellbar, dass durch die in den letzten Jahren sich deutlich veränderte Sprachkompetenz junger Menschen der vermutete Effekt des Ersterkrankungsalters als allgemeingültig anzusehen ist. Zudem greift der MWTB als Schätzer der prämorbiden Intelligenz nur eine Domäne der Intelligenz auf, nämlich erworbenes Wissen in Form von Vokabeln. Jedoch erlaubt er keine allumfassenden Rückschlüsse auf etwaige prämorbide kognitive Beeinträchtigungen. Somit kann eine ganz exakte Aussage über prämorbide Intelligenz und die Veränderung der Intelligenz über die Erkrankungsdauer nur durch longitudinales Testen vor bzw. im Krankheitsverlauf getroffen werden. Die definitive Assoziation des Ersterkrankungsalters und des damit zusammenhängenden prämorbiden Leistungsniveaus mit den in der vorliegenden Arbeit gefundenen kognitiven Leistungsunterschieden zwischen den männlichen $\Delta \mathrm{CP} \ddot{A} / \mathrm{J}$ Gruppen zum GRAS-Untersuchungszeitpunkt kann deshalb nicht gänzlich geklärt werden. Das in der vorliegenden Arbeit getestete „Modell“ Alter* $\Delta \mathrm{CP} \ddot{A} / \mathrm{J}$-Gruppe sagt aus, ob die Einflussgrößen „Alter“ und die $\Delta \mathrm{CPÄ-Gruppenzugehörigkeit} \mathrm{einen} \mathrm{gemeinsamen,} \mathrm{synergistischen}$ Effekt auf die kognitiven Ergebnisse ausüben. Da in der vorliegenden Arbeit jedoch die Assoziation von Dosissteigerung mit der Kognition weitestgehend isoliert betrachtet werden sollte, wurde in den ANCOVAs der „Faktor“ $\triangle \mathrm{CPÄ/J-Gruppe} \mathrm{gesondert} \mathrm{betrachtet.} \mathrm{Somit} \mathrm{be-}$ schreibt der „Faktor“ die vom Alter unabhängige Assoziation der $\Delta \mathrm{CP} \ddot{\mathrm{J}}$ Gruppenzugehörigkeit mit den kognitiven Leistungstests. Unter „Faktor im Modell“ wurde gezeigt, dass die antipsychotische Dosissteigerung pro Jahr unabhängig vom Alter in der Stichprobe der Männer signifikant mit Wahrnehmung, Aufmerksamkeit, visuomotorische Geschwindigkeit, Feinmotorik, Verarbeitungsgeschwindigkeit, Konzentration und Lernen assoziiert war. Diese Unterschiede erwiesen sich auch in der Stichprobe der Frauen als signifikant, in welcher zusätzlich Unterschiede im auditiven Arbeitsgedächtnis, in schlussfolgerndem Denken, und kognitiver Flexibilität nachgewiesen werden konnten. So ist die antipsychotische Dosissteigerung über eine langjährige Applikationsdauer in beiden Geschlechtern negativ mit dem kognitiven Funktionsniveau assoziiert. Damit konnte Hypothese 4 auch unter Berücksichtigung der bedeutendsten Störgröße Alter angenommen werden. Zwar war das Alter mit kognitiver Leistung und antipsychotischer Dosissteigerung assoziiert, jedoch bedingte der 
Faktor $\Delta \mathrm{CPÄ/J-Gruppe} \mathrm{unabhängig} \mathrm{von} \mathrm{der} \mathrm{Beeinflussung} \mathrm{des} \mathrm{Alters} \mathrm{kognitive} \mathrm{Unterschiede}$ zwischen den Probanden.

Ob letztlich die Gruppeneinteilung nach antipsychotischer Dosissteigerung pro Medikationsjahr oder die in den Gruppen unterschiedliche Rate an Probanden mit extremen antipsychotischen Tagesdosen zu den deutlichen kognitiven Gruppendifferenzen geführt hat, ist nicht eindeutig festzustellen. Da eine hohe Dosissteigerung pro Jahr letztlich zu hohen antipsychotischen Gesamttagesdosen führt, sind diese beiden Variablen stark miteinander assoziiert. In beiden Geschlechtern war die Anzahl der Probanden mit antipsychotischen Tagesdosen $>1000 \mathrm{CP} \ddot{\text { in }}$ den $\Delta \mathrm{CP} \ddot{\mathrm{A}} / \mathrm{J} \uparrow$-Gruppe signifikant höher als in den $\Delta \mathrm{CP} \ddot{\mathrm{A}} / \mathrm{J} \downarrow$-Gruppen. Es wird davon ausgegangen, dass hohe antipsychotische Tagesdosen $(\geq 1000$ CPÄ/d) weder zur Verbesserung der Psychopathologie noch der Kognition führen (Harvey et al. 2004; Lipkovich et al. 2008). Hori et al. konnten zeigen, dass Patienten mit Tagesdosen $>1000 \mathrm{CPÄ/d}$ signifikant schlechtere Ergebnisse in den kognitiven Einheiten visuelles Gedächtnis, Wortgedächtnis, Exekutivfunktionen und praktischer IQ erreichten, was nicht durch die Krankheitsschwere erklärt werden konnte (Hori et al. 2006). Kongruent wurde in einer kleinen Interventionsstudie die antipsychotische Tagesdosis von extremen Werten reduziert, worunter die Probanden signifikant bessere Leistungen in den Exekutivfunktionen im Vergleich zur Kontrollgruppe zeigten (Kawai et al. 2006). Die Ergebnisse der vorgelegten Arbeit weisen in beiden Geschlechtern Defizite dieser kognitiven Domänen der beiden $\Delta \mathrm{CPÄ/J \uparrow -Gruppen} \mathrm{im}$ Vergleich zu den $\Delta$ CPÄ/J $\downarrow$-Gruppen auf. Harrow und Jobe (2007) konnten in einer 15jährigen follow-up Studie zeigen, dass ein Teil schizophrener Patienten ohne AP einen besseren Verlauf darbot als jener mit AP. Jedoch unterschieden sich medizierte Patienten und Patienten ohne antipsychotische Medikation in wesentlichen Parametern wie prämorbidem Leistungsniveau, sozialer Festigung, Copingstrategien, Belastbarkeit und innere Ressourcen, sodass eine eindeutige Aussage über eine etwaige negative Wirksamkeit der AP nicht getroffen werden konnte (Harrow und Jobe 2007). Untersuchungen, die einen negativen Effekt der antipsychotischen Dosissteigerung auf das kognitive Leistungsniveau bei Schizophrenen nachweisen und Mechanismen dieses Phänomens erklären, lagen zum Zeitpunkt der Erstellung dieser Arbeit nicht vor. Bekannt ist jedoch, dass zentraler Dopaminmangel, wie er unter antipsychotischer Therapie (vgl. Seite 7 f.) oder bei (nicht dementen) Parkinsonpatienten auftritt, zu kognitiven Defiziten führen kann (Pillon et al. 2003). Es ist ethisch nicht vertretbar, Probanden sehr hohen antipsychotischen Dosen auszusetzen um etwaige neurotoxische Effekte von AP zu untersuchen. Ebenso wenig ist es vertretbar, Schizophrenen antipsychotische Therapie vorzuenthalten, um differenzieren zu können, welche Prozesse AP- oder krankheitsbedingt sind. So sollen im Folgenden tierexperimentelle Studien aufgeführt werden, um 
etwaige Pathomechanismen kognitiver Verschlechterung unter antipsychotischer Therapie darzustellen.

Bei Ratten und Affen erwiesen sich Korrelate des Arbeitsgedächtnisses und der Aufmerksamkeit unter Medikation mit Clozapin, Haloperidol, Olanzapin, Risperidon oder Ziprasidon als beeinträchtigt (Didriksen 1995; Skarsfeldt 1996; Addy und Levin 2002). Dorph-Petersen et al. (2005) konnten im histologischen Präparat von Makaken eine Assoziation der Abnahme von grauer Hirnsubstanz und chronischer Haloperidol- und Olanzapinapplikation nachweisen. Sowohl das Hirngewicht, als auch die Gehirngröße stellten sich bei Makaken, die über 17-27 Monate mit Haloperidol oder Olanzapin behandelt wurden, signifikant geringer dar als in der mit Placebo behandelten Kontrollgruppe (Dorph-Petersen et al. 2005). Die Abnahme grauer Substanz wurde vor allem auf einen Schwund an Astrozyten (20,5\%) und Oligodendrozyten $(12,9 \%)$ erklärt (Konopaske et al. 2008). Oligodendrozyten myelinisieren Axone und ermöglichen so eine physiologische Kommunikation der Nervenzellen. Astrozyten sind essentiell für die Homeostase der Neurotransmitter, besonders des Glutamats. Somit sind beide Zelltypen Grundlage zentraler Hirnfunktionen wie auch kognitiver Leistungen (Davis et al. 2003; Hertz 2004; Tamminga et al. 2010). Die tierexperimentellen Ergebnisse mit Olanzapin und Haloperidol können nicht generalisiert werden, da AP deutlich unterschiedliche Eigenschaften und Bindungsprofile aufweisen. Zum anderen können Ergebnisse aus Tierexperimenten nicht auf den Menschen übertragen werden, wenngleich sie wichtige Tendenzen anzeigen können. So kann lediglich vermutet werden, dass eine lang andauernde, steigende und schließlich hochdosierte antipsychotische Therapie zu direkten negativen Effekten der Integrität von Nervenzellen führen kann und dadurch das kognitive Funktionsniveau auch bei Menschen negativ beeinflusst werden kann. Eine kürzlich veröffentliche longitudinale MRI-Studie konnte jedoch nachweisen, dass sowohl die Dauer, als auch die Höhe der antipsychotische Therapie unabhängig von den Einflussgrößen Erkrankungsdauer, Krankheitsschwere und Substanzabhängigkeit zu einer Reduktion der weißen und grauen Substanz des ZNS führen (Ho et al. 2011). Abgesehen von strukturellen Veränderungen des ZNS durch AP konnte bei Gesunden unter AP-Applikation eine spontane „Zunahme“ der Negativsymptomatik festgestellt werden. So stellten erfahrene Psychiater in einer doppel-verblindeten Studie bei gesunden Probanden 3 Stunden nach Applikation von Haloperidol und Risperidon eine Zunahme der Negativsymptomatik fest (Artaloytia et al. 2006). Als Ursache wurde die Sedierung mit einhergehender subjektiv empfundener Benommenheit diskutiert. Dieser Aspekt kann auch die $\Delta \mathrm{CPÄ} / \mathrm{J}$ Gruppenunterschiede in der kognitive Leistung bedingen, da mit einer steigenden Dosierung vor allem niedrig potenter AP ebenfalls eine steigende Sedierung angenommen werden kann (Kane und Sharif 2008). Letztlich kann zwar die Assoziation von Steigerung der antipsychotischen Tagesdosis über die Medikationsdauer mit kognitivem Defizit dargestellt werden, je- 
doch unterscheiden sich die jeweils 2 verglichenen $\Delta \mathrm{CPÄ/J} \mathrm{-Gruppen} \mathrm{in} \mathrm{wesentlichen} \mathrm{Para-}$ metern der Erkrankungsschwere. Die Diskussion der Assoziation von $\Delta \mathrm{CP} \ddot{\mathrm{A}} / \mathrm{J}$ mit der Erkrankungsschwere und kognitiver Beeinträchtigung erfolgt in Kap. 4.6.

\subsection{Dosissteigerung und Nebenwirkungen}

Es ist bekannt, dass antipsychotische Dosissteigerung zu hohen antipsychotischen Tagesdosen führt und diese mit einem breiten Spektrum an Nebenwirkungen assoziiert sind (Stahl und Grady 2004). So zeigten auch in der untersuchten Stichprobe Probanden der $\Delta \mathrm{CP} \ddot{A} / \mathrm{J} \uparrow-$ Gruppen eine höhere Rate an neurologischen Medikamentennebenwirkungen als Probanden der Vergleichsgruppen. Bei den Männern erreichten die Paarkontraste in den Bereichen Akathisie (körperliche Unruhe) und tardive Dyskinesie Signifikanz, bei den Frauen in den Bereichen muskuläre Rigidität und tardive Dyskinesie. Hypothese 5 wurde daher angenommen.

Hinsichtlich der Ergebnisse der subjektiv geäußerten Nebenwirkungen konnten zwischen den $\Delta \mathrm{CPÄ/J-Gruppen} \mathrm{keine} \mathrm{bedeutsamen} \mathrm{Unterschiede} \mathrm{festgestellt} \mathrm{werden.} \mathrm{Die} \mathrm{Fallzahlen} \mathrm{be-}$ trachtend wird deutlich, dass nur eine begrenzte Anzahl von Probanden von sich aus APNebenwirkungen geäußert hat. Die Tatsache kann durch die Schwere der Erkrankung bedingt sein. Schwerer erkrankte Schizophrene zeigen zum einen starke Einschränkungen in der Körperwahrnehmung und können zum anderen schwerer zu der untersuchenden Person in Kontakt treten und über etwaige Beeinträchtigungen berichten (Scharfetter 1996; Waters und Badcock 2010). Die Differenzen zwischen den Dosissteigerungsgruppen von psychopathologischer und auch allgemeiner Beeinträchtigung unterstützen diese Vermutung.

Es kann darauf geschlossen werden, dass schwerer erkrankte Probanden eine größere Dosissteigerung erfahren haben, welche kognitive, psychopathologische und allgemeinfunktionelle Defizite nicht kompensieren konnte und zudem zu einer höheren Rate an Nebenwirkungen geführt hat. Tardive Dyskinesien sind irreversibel und schränken die Betroffenen durch direkte motorische Ausfallerscheinungen und/oder eine größere Stigmatisierung persönlich und sozial ein. Diese Einschränkungen können sich über Dauer jedoch wiederum auch direkt auf das kognitive Leistungsniveau auswirken, da die Betroffenen weniger am „normalen“ und sozialen Leben teilnehmen und in der Isolation weniger geistig gefordert werden (Bartels SJ und Pratt 2009; van Zelst 2009). Die alltäglichen geistigen Herausforderungen stellen eine „regelmäßige Übung“ dar, um kognitiv belastbar zu bleiben und einem Abbau nach Möglichkeit vorzubeugen. Folglich macht dieser Zusammenhang darauf aufmerksam, dass die als kognitionsfördernd eingesetzten AP bei steigender und hoher Dosierung durch Nebenwir- 
kungen und dadurch bedingte Konsequenzen wie Stigmatisierung das Gegenteil, nämlich eine zusätzliche kognitive Beeinträchtigung verursachen können.

\subsection{Dosissteigerung und Komedikation}

Die Tatsache, dass die $\Delta \mathrm{CP} \ddot{A} / \mathrm{J} \uparrow-G r u p p e n$ mehr EPMS zeigten als die $\Delta \mathrm{CP} \ddot{\mathrm{A}} / \mathrm{J} \downarrow$-Gruppen, erklärt auch die beobachteten Unterschiede in der Komedikation mit Anticholinergika. In beiden Geschlechtern wurden in den $\Delta \mathrm{CP} \ddot{\mathrm{A}} / \mathrm{J} \uparrow$-Gruppen signifikant mehr Anticholinergika und Benzodiazepine verabreicht als in der $\Delta \mathrm{CPÄ/J \downarrow -Gruppe.} \mathrm{Somit} \mathrm{konnte} \mathrm{Hypothese} 6$ angenommen werden. Die Einnahme von Anticholinergika führt zu schlechteren kognitive Leistungen der Domänen Lernen, Gedächtnis und Interferenzleistungen (Spohn und Strauss 1989; Vinogradov et al. 2009). Demzufolge können bei den Männern der signifikante Unterschied im ZST, sowie bei den Frauen die signifikanten Unterschiede in den Tests BZT, TMTb, VLMT und ZST zwischen den $\triangle \mathrm{CPÄ/J-Gruppen} \mathrm{möglicherweise} \mathrm{auch} \mathrm{auf} \mathrm{die} \mathrm{Unter-}$ schiede in der Applikationsrate der Anticholinergika zurückzuführen sein. Die Notwendigkeit der Anticholinergika ist wiederum in der Steigerung bzw. einer hohen antipsychotischen Tagesdosis begründet. Somit hängen diese beiden Größen untrennbar zusammen und eine Aussage über das spezifische Ausmaß der Kognitionsbeeinträchtigung jeder einzelnen Größe ist nicht möglich. Jedoch kann von einem negativ-synergistischen Effekt auf die Kognition ausgegangen werden.

Da vor allem die Kognition das allgemeine und alltägliche Leistungsniveau der Schizophrenen bedingt (Green 1996 b; Green et al. 2004), ist eine Dosissteigerung, die eine Komedikation mit Anticholinergika nach sich zieht, aufgrund der negativen Auswirkungen auf die Kognition kritisch zu betrachten. Ebenfalls wurden in der untersuchten Stichprobe Benzodiazepine in den $\Delta \mathrm{CP} \ddot{\mathrm{A}} / \mathrm{J} \uparrow$-Gruppen häufiger verabreicht. Benzodiazepine beeinträchtigen die kognitiven Domänen Arbeitsgedächtnis, Lernen, Aufmerksamkeit, Entscheidungsfindung und psychomotorische Geschwindigkeit negativ (Kleinknecht und Donaldson 1975; Barker et al. 2004). So kann nicht ausgeschlossen werden, dass die signifikant höhere Rate an mit Benzodiazepinen behandelten Probanden in der $\Delta \mathrm{CPÄ} / \mathrm{J} \uparrow$-Gruppe beider Geschlechter die kognitiven Gruppenunterschiede mitbedingt hat. Die signifikanten Unterschiede zwischen den Dosissteigerungsgruppen bei den Männern in den Tests Alertness und ZST, sowie bei den Frauen in den Tests Alertness, BZT, Dotting, LPS3, Tapping, TMTa, TMTb, VLMT, ZST und Summenwert durch die Applikationsraten der Benzodiazepine mitbegründet sein. Allerdings ist festzustellen, dass wegen der geringen Anzahl der mit Benzodiazepinen und/oder Anticholinergika behandelten Probanden innerhalb der großen $\Delta \mathrm{CPÄ/J-Gruppen} \mathrm{die} \mathrm{kognitiven} \mathrm{Un-}$ terschiede nicht hinreichend durch die Komedikation erklärt werden können (vgl. Tab. 18). 
Nichtsdestotrotz darf dieser Aspekt in der Gesamtbetrachtung der vorliegenden Daten nicht gänzlich außer Acht gelassen werden.

\subsection{Dosissteigerung und Krankheitsschwere}

Hypothese 7 wurde angenommen, da im direkten Vergleich der $\Delta \mathrm{CPÄ/J-Gruppen} \mathrm{beider}$ Geschlechter Probanden mit höherer Dosissteigerung pro Jahr über die gesamte Medikationszeit schwerer erkrankt waren, als solche mit moderater Dosissteigerung. In der vorliegenden Arbeit wurden als Maß der Krankheitsschwere die kumulative Dauer der sationären Aufenthalte, die psychopathologische Beeinträchtigung, die Beeinträchtigung im allgemeinen Funktionsniveau und neurologische Ausfallerscheinungen untersucht (vgl. Seiten 2 f. und 30). Die kognitive Beeinträchtigung als Kernsymptom der Schizophrenie ist als wichtige Messgröße der Krankheitsschwere zu verstehen. Auf die Assoziation mit der Dosissteigerung wurde auf Seite $65 \mathrm{f}$. hingewiesen.

Ziel der medikamentösen Therapie ist es, die Erkrankungsschwere zu lindern oder im besten Fall die Erkrankung zu heilen. In großen Studien konnte verfügbaren APs bislang jedoch nur eine geringe Wirksamkeit auf die Erkrankungsschwere nachgewiesen werden (Lieberman et al. 2005; Leucht et al. 2009 a). Ebenfalls besteht bislang keine eindeutige Meinung darüber, ob eine schwerwiegendere psychopathologische Beeinträchtigung als Zeichen einer schwerwiegenderen Erkrankung eine Steigerung der antipsychotischen Tagesdosis notwendig werden lassen sollte (Lipkovich et al. 2008). Die Ergebnisse der vorliegenden Arbeit zeigen jedoch deutlich, dass eine höhere Dosissteigerung pro Medikationsjahr bei beiden Geschlechtern mit den Parametern der Erkrankungsschwere assoziiert ist. Ito et al. (2005) stellten in einer Untersuchung der antipsychotischen Verschreibungspraxis von Psychiatern fest, dass eine persistierende Beeinträchtigung schwer erkrankter Patienten über den Erkrankungsverlauf hinweg eine Ursache für Polypharmazie und hohe antipsychotische Tagesdosen darstellt (Ito et al. 2005). So ist auf den Zusammenhang zu schließen, dass diejenigen Probanden, die einen längeren und schwerwiegenderen Krankheitsverlauf aufweisen, mehr antipsychotische Medikation verschrieben bekamen und deshalb vorrangig in der jeweiligen $\Delta \mathrm{CP} \ddot{\mathrm{C}} / \mathrm{J} \uparrow$-Gruppe kumuliert sind. Als wichtiger Schätzwert der Krankheitsschwere kann die kumulative Gesamtdauer psychiatrischer Krankenhausaufenthalte angesehen werden (vgl. Seite 30). In dieser Größe ist sowohl die Dauer der aktiven Erkrankung zusammengefasst, als auch die zunehmende Krankheitsverschlechterung bei jeder neuen Erkrankungsexazerbation (Breier et al. 1991; Hoffman und McGlashan 1993; Harvey et al. 2010). In beiden Geschlechtern weist die $\Delta \mathrm{CP} \ddot{A} / \mathrm{J} \uparrow-G r u p p e$ eine signifikant längere kumulative Gesamtdauer psychiatrischer Krankenhausaufenthalte auf als die $\Delta \mathrm{CPÄ} / \mathrm{J} \downarrow$ Gruppen. Deshalb sind die Probanden der $\Delta \mathrm{CP} \ddot{\mathrm{A}} / \mathrm{J} \uparrow-$ Gruppen als schwerer erkrankt zu be- 
trachten. Harvey et al. (2010) konnten nachweisen, dass lang andauernde stationärpsychiatrische Aufenthalte mit einer Verschlechterung im neuropsychologischen, sozialen und allgemeinen Funktionsniveau assoziiert sind (Harvey et al. 2010). Diese Assoziation konnte in der vorliegenden Arbeit bestätigt werden. Im Folgenden soll einzeln auf die Dimensionen der Krankheitsschwere eingegangen werden.

Die Psychopathologie als wesentliche klinische Äußerungsform der Schizophrenie war in beiden Geschlechtern in der $\Delta \mathrm{CP} \ddot{A} / \mathrm{J} \uparrow-G r u p p e$ signifikant stärker ausgeprägt als in der $\triangle \mathrm{CPÄ/J \downarrow -Gruppe.} \mathrm{Dies} \mathrm{äußerte} \mathrm{sich} \mathrm{in} \mathrm{höheren} \mathrm{Werten} \mathrm{aller} \mathrm{Subskalen} \mathrm{der} \mathrm{PANSS.} \mathrm{Die} \mathrm{all-}$ gemeine Psychopathologie sowie die Positivsymptomatik schränken in Form von z.B. Halluzinationen und Wahnsymptomatik, Angst oder sozialem Rückzug die Betroffenen im sozialen Umgang ein. Die kognitive Beeinträchtigung auf breiter Basis konnte jedoch bislang nicht mit der Positivsymptomatik oder der allgemeinen Psychopathologie assoziiert werden (Dominguez Mde et al. 2009). Somit ist die $\Delta \mathrm{CPÄ/J \uparrow -Gruppe} \mathrm{zwar} \mathrm{aus} \mathrm{der} \mathrm{Sicht} \mathrm{der} \mathrm{Positivsympto-}$ matik und allgemeinen Psychopathologie stärker erkrankt, jedoch kann diese das schlechtere kognitive Funktionsniveau der $\Delta \mathrm{CPÄ/J \uparrow -Gruppe} \mathrm{nicht} \mathrm{erklären.} \mathrm{Hingegen} \mathrm{ist} \mathrm{bewiesen,} \mathrm{dass}$ die Negativsymptomatik mit kognitiver Leistungsabnahme vor allem in den Bereichen Lernen, Gedächtnis, Aufmerksamkeit und gesamt IQ assoziiert ist (Dominguez Mde et al. 2009; Ventura et al. 2009). Somit können die Unterschiede der kognitiven Leistungen zwischen den $\Delta \mathrm{CP} \ddot{\mathrm{A}} / \mathrm{J}$-Gruppen beider Geschlechter durch das höhere Maß an Negativsymptomatik als Zeichen eines schwereren Erkrankungsverlaufes in den $\Delta \mathrm{CP} \ddot{\mathrm{A}} / \mathrm{J} \uparrow-\mathrm{Gruppen}$ als ursächlich angesehen werden. Nicht gänzlich außer Acht gelassen werden darf jedoch die Tatsache, dass sich die (sedierende) Wirkung verschiedener AP häufig als sogenannte sekundäre Negativsymptomatik manifestiert, bzw. als solche interpretiert wird (Artaloytia et al. 2006; Buckley und Stahl 2007). Zwar kann keine definitive Aussage über die zu Grunde liegende Einflussgröße kognitiver Leistung getroffen werden, jedoch darf sowohl auf eine Assoziation von Krankheitsschwere und $\triangle \mathrm{CPÄ/J}$, als auch auf eine Assoziation von $\Delta \mathrm{CP} \ddot{\mathrm{A}} / \mathrm{J}$ und kognitiver Beeinträchtigung geschlossen werden.

Die größere Erkrankungsschwere in den $\Delta \mathrm{CP} \ddot{\mathrm{A}} / \mathrm{J} \uparrow$-Gruppen konnte auch durch die statistische Auswertung der globalen Einschätzung des Gesundheitszustandes mittels CGI und der Bewertung des allgemeinen Funktionsniveaus mit Hilfe des GAF verdeutlicht werden. Das Untersuchungsinstrument CGI ist als globaler Schätzer zu verstehen, da, ohne dass exemplarisch Kriterien genannt werden, Patienten unterschiedlichster Erkrankungsbilder auf einer $7-$ stufigen Skala in der Erkrankungsschwere eingeschätzt werden. So ist subjektiv schwer zu beurteilen, ob ein stark psychotischer Patient kranker ist als ein z.B. stark verlangsamter Patient. In Relation zu den bereits diskutierten Ergebnissen kann jedoch von einer glaubhaften Einschätzung mit Hilfe dieses Instrumentes ausgegangen werden. So bestätigte auch der sta- 
tistische $\triangle \mathrm{CPÄ/J-Gruppenvergleich} \mathrm{der} \mathrm{GAF-Skalenwerte} \mathrm{die} \mathrm{Annahme} \mathrm{einer} \mathrm{schwerwiegen-}$ deren Erkrankung in den $\Delta \mathrm{CPÄ/J \uparrow -Gruppen.} \mathrm{Die} \mathrm{GAF} \mathrm{erhebt} \mathrm{psychische,} \mathrm{soziale} \mathrm{und} \mathrm{beruf-}$ liche Einschränkungen, ohne dass körperliche oder umweltbedingte Einschränkungen einflieBen. Die $\Delta \mathrm{CP} \ddot{\mathrm{I}} / \mathrm{J}$-Gruppenunterschiede unterstützen die Theorie, dass kognitive Defizite als Ursache von Einschränkungen im allgemeinen Funktionsniveau Schizophrener gesehen werden können (vgl. Seite 3 f.). Die Probanden der $\Delta \mathrm{CP} \ddot{\mathrm{A}} / \mathrm{J} \uparrow-$ Gruppen sind deshalb in der Einschätzung des allgemeinen Funktionsniveaus schlechter, da sie aufgrund von kognitiver Beeinträchtigung größere Schwierigkeiten in der Bewältigung alltäglicher Probleme zeigen und weniger selbstständig leben können (Green 1996 a). So wird auch an dieser Stelle die Assoziation von Krankheitsschwere, Kognitiver Beeinträchtigung und $\Delta \mathrm{CP} \ddot{/} / \mathrm{J}$ deutlich.

Die mittels CNI erfassten neurologischen Ausfallerscheinungen der untersuchten Probanden können als Schätzer der hirnorganischen Integrität und Erkrankungsschwere interpretiert werden (Chen et al. 1995). So offenbart sich eine schwerere Verlaufsform der Schizophrenie in den $\Delta \mathrm{CP} \ddot{\mathrm{A}} / \mathrm{J} \uparrow-G r u p p e n$ nicht nur in einer schwerwiegenderen psychopathologischen und kognitiven Beeinträchtigung, sondern auch in einer größeren Anzahl neurologischer Defizite. In den $\triangle \mathrm{CPÄ/J \uparrow -Gruppen} \mathrm{beider} \mathrm{Geschlechter} \mathrm{wiesen} \mathrm{die} \mathrm{Probanden} \mathrm{höhere} \mathrm{Werte} \mathrm{in} \mathrm{dem}$ CNI-Summenwert auf als die Teilnehmer der $\Delta \mathrm{CPÄ/J \downarrow -Gruppen,} \mathrm{wobei} \mathrm{nur} \mathrm{der} \mathrm{Unterschied}$ in der Stichprobe der Frauen Signifikanz erreichte. Dieses Ergebnis ist vor dem Hintergrund zu verstehen, dass mittels CNI erhobene neurologische Defizit mit kognitiver Beeinträchtigung assoziiert sind (Liddle 1987). So spiegelt sich die generalisierte hirnmorphologische Veränderung Schizophrener sowohl auf motorischer Ebene in den „soft-signs“ wider, als auch in einer stärkeren Beeinträchtigung kognitiver Fähigkeiten. Der in der vorliegenden Arbeit in den statistischen Auswertungen genutzte Summenwert des CNI setzt sich sowohl aus neurologischen „soft-signs“ als auch „hard-signs“ zusammen (vgl. Seite 25). Da die Diagnose „Schizophrenie“ der untersuchten Probanden genau überprüft wurde und nur eindeutig schizophrene Probanden in die Auswertungen eingeschlossen wurden, sind fokale neurologische Defizite als Ursache der schizophrenen Symptomatik auszuschließen. Jedoch gehen in den neurologischen Summenwert des CNI auch Dyskinesien ein. Diese sind wiederum durch hohe antipsychotische Tagesdosen bedingt (vgl. Seite 72 f.). So sind die neurologischen Ausfallerscheinungen als Assoziation von Krankheitsschwere und hoher antipsychotischer Dosis, bzw. Dosissteigerung zu verstehen.

Schließlich konnte in der vorliegenden Arbeit eine Assoziation von hoher antipsychotischer Dosissteigerung, deutlicher kognitiver Beeinträchtigung und einem größeren Maß an Krankheitsschwere aufgezeigt werden. Die $\Delta \mathrm{CPÄ/J \uparrow -Gruppe} \mathrm{beider} \mathrm{Geschlechter} \mathrm{zeigte} \mathrm{im} \mathrm{Ver-}$ gleich zu der $\Delta \mathrm{CPÄ/J \downarrow -Gruppe} \mathrm{einen} \mathrm{signifikant} \mathrm{höheren} \mathrm{Grad} \mathrm{an} \mathrm{Erkrankungsschwere} \mathrm{in}$ 
den Variablen Gesamtdauer psychiatrischer Krankenhausaufenthalte, PANSS positiv, negativ und allgemein, CGI, GAF und CNI. In der Abbildung 12 ist der Zusammenhang bildlich dargestellt.

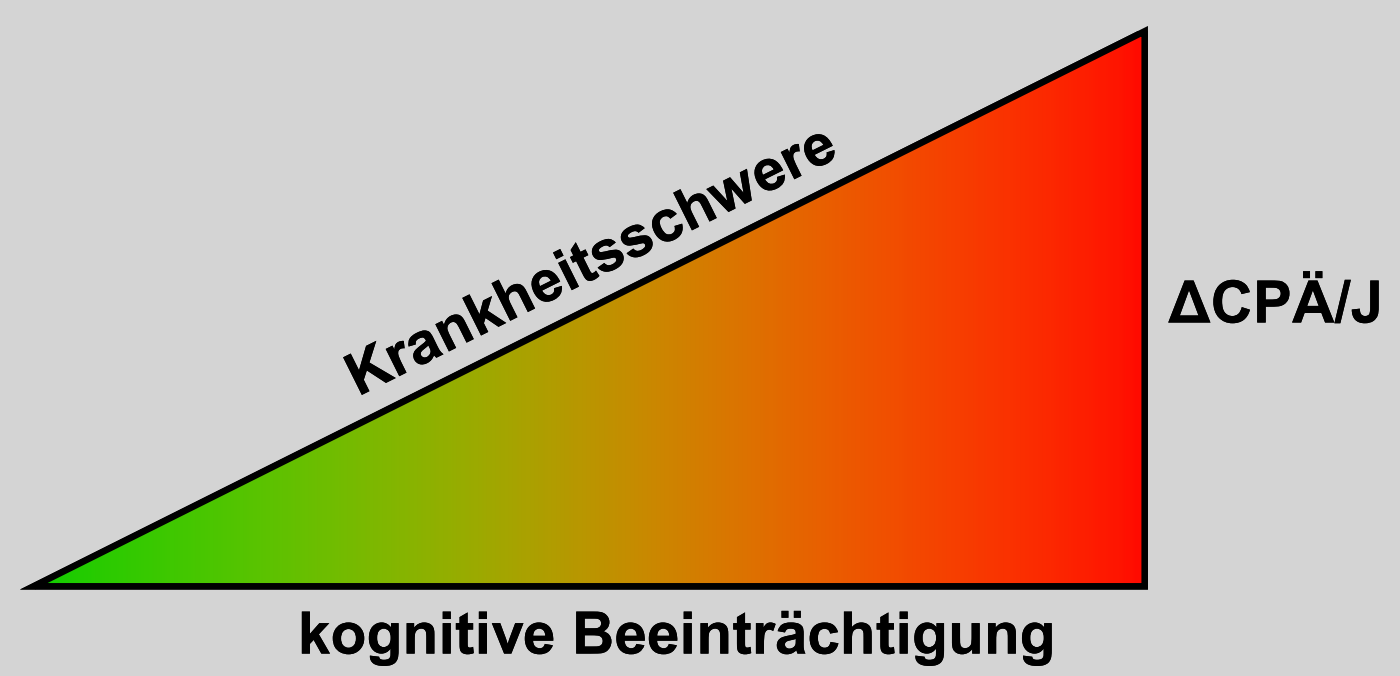

$\triangle C P A ̈:$ Steigerung der antipsychotischen Tagesdosis pro Jahr Medikationsdauer

Abbildung 12: Schematische Darstellung der Assoziation von Dosissteigerung pro Jahr Medikationsdauer, kognitiver Beeinträchtigung und Krankheitsschwere

Je schwerer die Schizophrenie bei einem Probanden ausgeprägt ist, desto größer ist dessen kognitive Beeinträchtigung, desto höher ist die dem Probanden zu Teil werdende antipsychotische Dosissteigerung pro Medikationsjahr. Je höher die antipsychotische Dosissteigerung eines Probanden, desto größer ist die Wahrscheinlichkeit extrapyramidaler Symptomatik und Notwendigkeit der Einnahme kognitionsmodulierender Komedikation.

Die kognitive Beeinträchtigung ist als Indikator der Krankheitsschwere anerkannt und wird bereits als diagnostisches Kriterium diskutiert (Keefe und Fenton 2007). Die vorliegenden Daten erlauben die Schlussfolgerung, dass auch das $\Delta \mathrm{CPÄ/J}$ als grobes, jedoch klinisch direkt verfügbares, Einschätzungsmaß der Erkrankungsschwere betrachtet werden kann.

\subsection{Stärken der vorliegenden Arbeit und klinische Relevanz der Befunde}

Die vorliegende Arbeit ist die erste, die die Assoziation von antipsychotischer Dosissteigerung mit dem kognitiven Leistungsniveau untersucht. Zum Zeitpunkt der Verfassung lagen nur wenige Studien vor, die die Höhe der antipsychotischen Therapie im Hinblick auf das kognitive Leistungsniveau kritisch betrachteten.

Ein Vorteil dieser Arbeit liegt in der genauen Charakterisierung der zu Grunde liegenden Stichproben. Es kann davon ausgegangen werden, dass die eindeutige Diagnose „Schizophre- 
nie“ in der untersuchten Stichprobe besser gesichert ist als in vergleichbaren Untersuchungen, da mit jedem Probanden das sehr reliable SKID-I durchgeführt wurde (Skre et al. 1991). Alle Untersuchungen wurden von ein- und demselben erfahrenen Untersuchungsteam erhoben und die Einschätzung von der Erkrankungsschwere wurde auf den verschieden Skalen von den mindestens 2 Untersuchern pro Proband im Konsens getroffen. Dieses Vorgehen hat positiven Einfluss auf die Objektivität und Reliabilität der Untersuchungsergebnisse. Da in der vorliegenden Studie das gesamte Spektrum des Krankheitsbildes, sowohl hinsichtlich der Typen, als auch hinsichtlich der Krankheitsstadien berücksichtigt wurde, ist von einer sehr umfassenden Stichprobe auszugehen. Aufgrund des Multicenter-Designs der Studie besteht die untersuchte Stichprobe aus Probanden vieler verschiedener Kliniken in Deutschland. Somit können die Ergebnisse besser generalisiert werden, da eventuelle Charakteristika bestimmter Kliniken in Patientenführung oder Medikation im Rahmen der großen Gesamtstichprobe weniger bedeutsam sind. Viele Studien, die die Wirksamkeit von AP auf die Kognition untersuchen legen zur Vergleichbarkeit feste Tagesdosen fest. Diese Tagesdosen sind meistens an antipsychotischen „Richt-Dosen“ orientiert. Citrome et al. (2005) konnten nachweisen, dass antipsychotische Tagesdosen im klinischen Alltag oftmals signifikant höher waren, als solche in klinischen Studien (Citrome et al. 2005). So spiegeln die in der GRAS-Studie erhobenen antipsychotischen Tagesdosen reale Werte wider, die frei von studienabhängigen Einflussfaktoren sind. Des Weiteren bildet die Querschnittsuntersuchung den Ist-Zustand des kognitiven Leistungsniveaus ab, ohne dass Placebo- oder Übungseffekte einfließen. Hervorzuheben ist, dass in der vorliegenden Arbeit ein sehr breites Spektrum kognitiver Leistungen untersucht wurde. Indem die GRAS-Untersuchungsbatterie kognitive Leistungen von Informationsverabeitungsgeschwindigkeit, Wahrnehmung und Aufmerksamkeit, Arbeitsgedächtnis, Lernen und schlussfolgerndes Denken untersucht, wurden 5 der 7 Kognitionsbereiche der maßgebenden MATRICS Consensus Cognitive Battery erfasst. Darüber hinaus wurde die motorische Geschwindigkeit gemessen, sowie das prämorbide Leistungsniveau geschätzt. Besonders die Schätzung des prämorbiden Leistungsniveaus ist für die Interpretation kognitiver Gruppenunterschiede in Querschnittsuntersuchungen wichtig, um Hinweise auf etwaige schon vor Beginn der Erkrankung bestehende kognitive Unterschiede zu erlangen. Auch ist die strukturierte Untersuchung von unerwünschten Arzneimittelwirkungen der AP mit Hilfe international anerkannter Untersuchungsinstrumente hervorzuheben. So wurden die verschiedenen Dyskinesien anhand spezifischer Skalen erhoben. Jedoch wurden die Nebenwirkungen der AP nicht nur fremd-, sondern auch von den Probanden in einer offenen Frage selbstbeurteilt. Die ausgeprägte Differenz zwischen diesen beiden Nebenwirkungseinschätzungen macht deutlich, inwieweit die Schizophrenie die Selbstwahrnehmung der Probanden beeinträchtigen kann. 
Im Fokus der vorliegenden Studie stand die antipsychotische Tagesdosis. Aufgrund starker interindividueller antipsychotischer Dosis- und Wirkstoffunterschiede wurde, um Vergleichbarkeit zu erreichen, für jeden Probanden die antipsychotische Tagesdosis in CPÄ umgerechnet. Für alle 47 oral und 12 in Depotform applizierten Wirkstoffe, auf die die Probanden unserer Studie eingestellt waren, wurde nach ausführlicher Literaturrecherche ein anerkannter Umrechnungsfaktor gefunden. So konnten in die Analysen Medikationen mit FGA, SGA und gemischter Art eingehen, sowie unterschiedliche Applikationsformen (oral, Depot, gemischt). Die große untersuchte Stichprobe ist aufgrund der Vergleichbarkeit eines sehr breiten Spektrums unterschiedlicher antipsychotischer Behandlungsregime als besonders zu betrachten. So war es aufgrund der großen Stichprobenanzahl möglich Subgruppen zu bilden. Da sich Männer und Frauen sowohl im kognitiven Profil unterscheiden, als auch hinsichtlich der Sensitivität gegenüber AP, wurden beide Geschlechter getrennt betrachtet um eine systematische Verzerrung der Ergebnisse zu vermeiden. Trotz der Aufteilungen gingen in die Analysen große Stichprobenanzahlen ein. Dadurch ist es unwahrscheinlich, dass einzelne Ausreißerwerte zu einem deutlichen Effekt auf das Ergebnis der Grundgesamtheit führen. So wirkte ebenfalls die Verwendung der Medianwerte, sowie der non-parametrischen Paartestung statistischen Effekten von Extremwerten entgegen. Schließlich wurde die Erkrankungsschwere mit verschiedenen Inventaren gemessen, was die Validität der Einschätzung der Erkrankungsschwere verbessert.

Trotz höherer Dosissteigerung pro Jahr lagen in den entsprechenden getesteten Stichproben schlechtere Werte in der Kognition, der Psychopathologie und eine höhere Anzahl an unerwünschten Arzneimittelwirkungen vor. Diese Befunde unterstreichen die Tatsache, dass bisher verfügbare AP keinen positiven Effekt auf die Kognition zeigen und somit den Erkrankungsstatus im Sinne eines besseren alltäglichen Funktionsniveaus nicht verbessern können. Aus der Assoziation von Krankheitsschwere und im Vergleich höherer antipsychotischer Dosissteigerung und Gesamttagesdosis könnte jedoch auch geschlossen werden, dass AP einen negativen Effekt auf Kognition und Erkrankungsschwere ausüben. Aus ethischen Gründen können selbstverständlich keine (Langzeit-) Studien mit unmedizierten Probanden, oder auch extrem hohen antipsychotischen Tagesdosen durchgeführt werden. Deshalb kann nicht geklärt werden, inwieweit die AP die kognitiven Leistungen bei Schizophrenen beeinträchtigen, oder in wie weit dieser Prozess allein der Krankheit geschuldet ist. Festzustellen ist aber, dass eine fortwährende Dosissteigerung über die Krankheitsdauer nicht zu gewünschten Verbesserungen führt. Vielmehr scheint eine solche Verschreibungspraxis die Rate an unerwünschten Arzneimittelwirkungen zu erhöhen, die Raten kognitionsbeeinträchtigender Komedikation zu erhöhen, zu Noncompliance führen und Stigmatisierung verursachen. Diese Aspekte führen 
letztlich wiederum zu einer scheinbaren Verschlechterung der Erkrankung, welche im Rahmen der Therapie oft eine weitere Dosissteigerung nach sich zieht. Somit besteht die Gefahr, dass durch die Erkrankung schwer beeinträchtigte Patienten durch hohe antipsychotische Dosissteigerung in eine Spirale geraten, die als krankheitsbedingte Destruktion interpretiert werden kann, ohne dass etwaige zusätzliche negative Aspekte der Medikation berücksichtigt werden. Eine Dosissteigerung der antipsychotischen Medikation ist auch deshalb kritisch zu bewerten, da gezeigt werden konnte, dass eine $\mathrm{D}_{2}$-R-Blockade von über $65 \%$ keine größere antipsychotische Wirkung zeigte, hingegen aber die Raten zum Teil lebensbedrohlicher internistischer Nebenwirkungen stiegen (Tauscher und Kapur 2001). In der vorliegenden Studie konnte ebenfalls gezeigt werden, dass Nebenwirkungen in Form von EPMS ebenfalls häufiger bei hoher Dosissteigerung pro Jahr auftreten. EPMS ziehen oft eine Komedikation mit Anticholinergika oder Benzodiazepinen nach sich, die ebenfalls das kognitive Funktionsniveau wieder verschlechtern. Die Ursachen, die Psychiater zu einer antipsychotischen Dosissteigerung verleiten, müssen ebenso untersucht und verstanden werden, wie die Metabolisierung und Rezeptorinteraktionen der AP, die eventuell zu einer Abnahme antipsychotischer Wirksamkeit über die Medikationsdauer führen kann. So sollte auch auf die möglichen destruktiven Eigenschaften der AP aufmerksam gemacht werden, sodass antipsychotische Dosissteigerungen im Bezug auf das Wohl des Patienten kritischer betrachtet werden und sehr hohe antipsychotische Dosen eine Ausnahme darstellen. Bei einer generellen Wirksamkeit von nur 41\% der AP muss über Alternativen nachgedacht, sowie an besser wirksamen und nebenwirkungsärmeren Substanzen geforscht werden. Antipsychotische Medikation ist im akuten Erkrankungsschub unabdingbar, jedoch konnten die bisher verfügbaren Präparate keine langfristige Besserung oder Heilung der Erkrankung erreichen. Der Schlüssel zur tatsächlichen Verbesserung der Erkrankung liegt in einer frühzeitigen Intervention und Kombination von medikamentöser Therapie, sozialpsychiatrischer Betreuung und kognitivem Training (Robinson et al. 1999 a; Burns et al. 2007; McGurk et al. 2007; Eack et al. 2010). Eack et al. (2010) konnten in einer 2-jährigen longitudinalen antipsychotisch unterstützten Studie nachweisen, dass Schizophrene, die regelmäßig kognitives Training und sozial-kognitive Übungen absolvierten, sowohl bessere kognitive Leistungen erzielten als auch eine geringere Abnahme der grauen Hirnsubstanz zeigten, als die Kontrollgruppe (Eack et al. 2010). Frühzeitige Intervention, intensivierte Betreuung und soziale Forderung der Schizophrenen führen zu einer verbesserten Compliance, die Krankheitsrückfälle verhindert (Robinson et al. 1999 b). Nur so kann bei den vor allem jungen Erkrankten eine regelrechte soziale Entwicklung und solide berufliche Ausbildung erreicht werden, die die Erkrankten im Alltag fordert, zu Selbstständigkeit führt und dem möglicherweise kognitiv-degenerativen Erkrankungsverlauf entgegenwirkt. Dies bestätigt eine Metaanalyse von McGurk et al. (2007), in der das Training kognitiver Fähigkeiten deut- 
lich mit Verbesserungen von Kognition, funktionalem Leistungsniveau im Alltag und Symptomverbesserung assoziiert war. Eine weitere wichtige Erkenntnis dieser Studie war, dass diese Verbesserungen besonders deutlich unter Begleitung psychiatrischer Rehabilitation auftraten, was auf einen synergistischen Effekt hinweist (McGurk et al. 2007). So scheint die Forderung und Förderung schizophrener Patienten ein wesentlicher Bestandteil der Therapie zu sein, der momentan häufig zu Gunsten der antipsychotischen Therapie und zu Lasten der Patienten vernachlässigt wird.

Die in der vorliegenden Arbeit gefundene Assoziation der antipsychotischen Dosissteigerung über die Medikationsdauer mit der Erkrankungsschwere und kognitiven Beeinträchtigung kann jedoch auch klinisch genutzt werden. So ist vorstellbar, anhand der Betrachtung von antipsychotischer Dosissteigerung über den Therapiezeitraum auf die Krankheitsschwere und kognitive Beeinträchtigung zu schließen. Wie praktikabel exakt eine solche Einschätzung letzten Endes ist, könnte mittels longitudinaler Beobachtung in Folgestudien geprüft werden.

\subsection{Kritische Diskussion der verwendeten Methoden und For- schungsausblick}

Zunächst ist darauf hinzuweisen, dass die in der vorliegenden Arbeit untersuchte Stichprobe nach Geschlecht aufgeteilt wurde, jedoch eine zusätzliche Randomisierung kognitionsmodulierender Einflussgrößen sowie Einflussgrößen antipsychotischer Tagesdosis wie Erkrankungsschwere, Komedikation, prämorbides Leistungsniveau und Raucherstatus nicht durchgeführt wurde. Diese hätte den Zusammenhang von Erkrankungsschwere und kognitivem Defizit klarer darstellen können. Da die Daten in einer Querschnittsstudie erhoben wurden, erlaubt die vorliegende Untersuchung keine definitiven Aussagen über die Kausalität des Zusammenhanges zwischen antipsychotischer Dosis/-steigerung und deren Korrelate. In folgenden Studien wäre ein longitudinales Design in Betracht zu ziehen, um die Ursachen der antipsychotischen Dosissteigerung z.B. im Hinblick auf Veränderungen der Erkrankungsschwere, Verschreibungspraktiken des behandelnden Psychiaters, Compliance und Veränderungen zu bestimmten Lebensaltern zu erfassen. Da longitudinale Studien jedoch die Problematik von Lern- und Placeboeffekten aufweisen, nicht das reale Dosierungsverhalten widerspiegeln und anfällig für Probandenfluktuationen sind, ist die Überlegenheit eines solchen Studiendesigns nicht sicher gegeben.

In der vorliegenden Studie wurde darauf aufmerksam gemacht, dass gleiche antipsychotische Tagesdosen aufgrund interindividueller Metabolisierungsunterschiede zu gleichen Blutplasmaspiegeln bei Schizophrenen führen können. So ist in Folgestudien darüber nachzudenken, ob die Blutplasmaspiegel zum einen eine bessere Untersuchungsgröße antipsychotischer Sen- 
sitivität darstellen und zum anderen als präzisere Einflussgröße der Kognition verwendet werden sollten. Da die vorliegende Arbeit erstmals die Assoziation antipsychotischer Dosissteigerung mit kognitiver Leistung darstellte, sind Folgestudien, die vorliegende Ergebnisse betätigen, wünschenswert. Weitergehend könnten die Ursachen von Enzyminduktion APverstoffwechselnder $\mathrm{CYP}_{450}$ sowie weiterer Einflussfaktoren untersucht werden, um die antipsychotische Therapie bei Schizophrenen besser anpassen zu können. In diesem Zusammenhang wäre auch über eine Entwicklung eines standardisierten Fragebogens nachzudenken, der die wichtigsten Einflussgrößen antipsychotischer Therapie wie z.B. Alter, Erkrankungsdauer, Rauchverhalten mittels zusammengefasster Werten in Relation zu „Standarddosen“ setzt, um behandelnden Psychiatern eine Hilfestellung bei der Dosierung an die Hand geben zu können. Auch machen die Ergebnisse der vorliegenden Arbeit darauf aufmerksam, dass auch hohe Dosissteigerungen die Krankheitsschwere nicht zufriedenstellend reduzieren können und zusätzlich zu beeinträchtigenden Nebenwirkungen führen und/oder eine beeinträchtigende Komedikation notwendig werden lassen. So muss in folgenden Studien die Grundlage der Verschreibungspraxis von AP behandelnder Psychiater verstanden werden. Durch Aufklärung über kontraproduktive Nebenwirkungen und alternative Behandlungskonzepte könnten schließlich extreme antipsychotische Tagesdosen mit fraglicher Krankheitsverbesserung vermindert werden.

Das wichtigste Ziel zukünftiger Forschung sollte jedoch sein, die Erkrankung Schizophrenie genauer zu verstehen, um eine wirksame Therapie zu finden, die möglichst frei von neurologischen, psychiatrischen und internistischen Nebenwirkungen ist und die Patienten von ihrem Leiden befreit. 


\section{ZUSAMMENFASSUNG}

Eine Verbesserung kognitiver Defizite schizophrener Patienten kann mit verfügbaren Antipsychotika nicht erreicht werden. Da die Verabreichung von Antipsychotika in der Praxis jedoch einen wesentlichen Teil des Behandlungskonzeptes darstellt, werden antipsychotische Tagesdosen oft auf sehr hohe Werte gesteigert, um das Erscheinungsbild der Krankheit zu verbessern. Wenig ist bislang über kognitive Beeinträchtigungen unter antipsychotischer Dosissteigerung zu sehr hohen Tagesdosen bekannt. Deshalb wurde in der vorliegenden Arbeit an einer umfassenden Stichprobe von 1029 Probanden die Assoziation der Höhe antipsychotischer Medikation über die Medikationszeit mit Kognition schizophrener Probanden unter Berücksichtigung des Geschlechts untersucht.

Es konnte gezeigt werden, dass Männer eine höhere antipsychotische Tagesdosis zum GRASUntersuchungszeitpunkt bekamen als Frauen, dieser Unterschied jedoch zum Zeitpunkt der Erstmedikation nicht bestand. Probanden beider Geschlechter erfuhren eine antipsychotische Dosissteigerung über die Medikationszeit, die Steigerung in der Stichprobe der Männer war jedoch höher als in der Frauenstichprobe. In der Stichprobe beider Geschlechter wurde ein Mediansplit der Größe $\Delta$ CPÄ/J durchgeführt. Die Gruppe geringerer Dosissteigerung wurde jeweils gegen jene höherer Dosissteigerung in Werten der Kognition, Medikamentennebenwirkungen, Komedikation und Erkrankungsschwere getestet. In paralleler Betrachtung beider Geschlechter zeigte die Gruppe höherer Dosissteigerung pro Medikationsjahr ( $\Delta \mathrm{CP} \ddot{A} / \mathrm{J} \uparrow)$ größere kognitive Defizite als die Gruppe geringerer Dosissteigerung $(\Delta \mathrm{CP} \ddot{\mathrm{A}} / \mathrm{J} \downarrow)$. Unter Berücksichtigung der wichtigsten kognitiven Einflussgröße Alter verdeutlichten sich die kognitiven Gruppenunterschiede. Zusätzlich zeigten die statistischen Auswertungen eine Assoziation von $\Delta \mathrm{CP} \ddot{\mathrm{A}} / \mathrm{J} \uparrow$-Gruppenzugehörigkeit mit einer höheren Rate an Nebenwirkungen, Komedikation sowie einer größeren Erkrankungsschwere. Wesentliche Einflussgrößen betrachteter Variablen wurden ausführlich diskutiert.

Aufgrund des nicht-experimentellen Querschnittsdesigns der zugrundeliegenden Studie konnte keine definitive Schlussfolgerung über den kausalen Zusammenhang zwischen Höhe antipsychotischer Dosissteigerung, kognitivem Leistungsniveau und Krankheitsschwere getroffen werden. Vorgelegte Ergebnisse lassen jedoch darauf schließen, dass die antipsychotische Dosissteigerung als Einschätzungsparameter der Krankheitsschwere verstanden werden kann. 


\section{ANHANG}

\section{Anhang A: Übersicht über die teilnehmenden Zentren der GRAS- Studie}

\begin{tabular}{|c|c|c|}
\hline Ort & Klinik & Leitung \\
\hline Bad Zwischenahn & $\begin{array}{l}\text { Karl-Jaspers-Klinik, Psychiatrieverbund } \\
\text { Oldenburger Land }\end{array}$ & $\begin{array}{l}\text { Marianne Becker-Emner } \\
\text { Dunja Hinze-Selch }\end{array}$ \\
\hline Bonn & $\begin{array}{l}\text { Klinik und Poliklinik für Psychiatrie und } \\
\text { Psychotherapie, Universitätsklinikum Bonn }\end{array}$ & Wolfgang Maier \\
\hline Eltville-Eichberg & $\begin{array}{l}\text { Vitos Klinik für forensische Psychiatrie, } \\
\text { Eltville }\end{array}$ & $\begin{array}{l}\text { Sara Gonzalez Cabeza } \\
\text { Roland Freese }\end{array}$ \\
\hline Fulda & $\begin{array}{l}\text { Klinik für Psychiatrie und Psychotherapie, } \\
\text { Klinikum Fulda }\end{array}$ & $\begin{array}{l}\text { Adelheid Czernik } \\
\text { Georg Wiedemann }\end{array}$ \\
\hline Gießen & $\begin{array}{l}\text { Vitos Klinik für forensische Psychiatrie, } \\
\text { Gießen }\end{array}$ & Rüdiger Müller-Isberner \\
\hline Göttingen & $\begin{array}{l}\text { Klinik für Psychiatrie und Psychotherapie, } \\
\text { Universitätsmedizin Göttingen }\end{array}$ & $\begin{array}{l}\text { Peter Falkai } \\
\text { Eckart Rüther }\end{array}$ \\
\hline Günzburg & $\begin{array}{l}\text { Klinik für Psychiatrie, Psychotherapie und } \\
\text { Psychosomatik, Bezirkskrankenhaus Günz- } \\
\text { burg }\end{array}$ & Thomas Becker \\
\hline Hofgeismar & $\begin{array}{l}\text { Vitos Klinik für Psychiatrie und Psychothe- } \\
\text { rapie, Merxhausen, Hofgeismar }\end{array}$ & Andreas Mielke \\
\hline Ingolstadt & $\begin{array}{l}\text { Zentrum für psychische Gesundheit, Klini- } \\
\text { kum Ingolstadt }\end{array}$ & Thomas Pollmächer \\
\hline Kassel & $\begin{array}{l}\text { Vitos Klinik für Psychiatrie und Psychothe- } \\
\text { rapie Kassel, Merxhausen }\end{array}$ & Rolf Günther \\
\hline Kiel & $\begin{array}{l}\text { Klinik für Psychiatrie und Psychotherapie, } \\
\text { Zentrum für Integrative Psychiatrie Kiel }\end{array}$ & Josef B. Aldenhoff \\
\hline Langenhagen & $\begin{array}{l}\text { Klinik für Psychiatrie und Psychotherapie, } \\
\text { Klinikum Region Hannover }\end{array}$ & Gunther Kruse \\
\hline Liebenburg & Dr. K. Frontheim Privat-Nerven-Klinik & Frank-Gerald Pajonk \\
\hline Lübbecke & $\begin{array}{l}\text { Klinik für Psychiatrie und Psychotherapie, } \\
\text { Krankenhaus Lübbecke }\end{array}$ & Udo Schneider \\
\hline $\begin{array}{l}\text { Merxhausen- } \\
\text { Bad Emstal }\end{array}$ & $\begin{array}{l}\text { Klinik für Psychiatrie und Psychotherapie, } \\
\text { Merxhausen, Bad Emstal }\end{array}$ & $\begin{array}{l}\text { Heinrich Kunze } \\
\text { Michael Franz }\end{array}$ \\
\hline Moringen & $\begin{array}{l}\text { Klinik für forensische Psychiatrie, Nieder- } \\
\text { sächsisches Landeskrankenhaus Moringen }\end{array}$ & $\begin{array}{l}\text { Martin Schott } \\
\text { Dirk Hesse }\end{array}$ \\
\hline Mühlhausen & $\begin{array}{l}\text { Klinik für Psychiatrie und Psychotherapie, } \\
\text { Ökumenisches Hainich Klinikum Mühlhau- } \\
\text { sen }\end{array}$ & Lothar Adler \\
\hline Rickling & $\begin{array}{l}\text { Psychiatrisches Zentrum, Psychiatrisches } \\
\text { Krankenhaus Rickling }\end{array}$ & $\begin{array}{l}\text { Hans-J. Schwarz } \\
\text { Wolfram Schreiber }\end{array}$ \\
\hline Rieden & $\begin{array}{l}\text { Reha-Zentrum für junge Abhängige, } \mathrm{AHG} \\
\text { Klinik am Waldsee }\end{array}$ & $\begin{array}{l}\text { Frank Tuchtenhagen } \\
\text { Frank Löhrer }\end{array}$ \\
\hline Rostock & $\begin{array}{l}\text { Klinik und Poliklinik für Psychiatrie und } \\
\text { Psychotherapie, Universität Rostock }\end{array}$ & $\begin{array}{l}\text { Jacqueline Höppner } \\
\text { Sabine Herpertz }\end{array}$ \\
\hline Taufkirchen & $\begin{array}{l}\text { Fachkrankenhaus für Psychiatrie, Psychothe- } \\
\text { rapie und Psychosomatische Medizin, Isar- } \\
\text { Amper-Klinikum }\end{array}$ & Matthias Dose \\
\hline Wilhelmshaven & $\begin{array}{l}\text { Klinik für Psychiatrie und Psychotherapie, } \\
\text { Reinhard-Nieter Krankenhaus }\end{array}$ & Here Folkerts \\
\hline Wunstorf & $\begin{array}{l}\text { Klinik für Psychiatrie und Psychotherapie, } \\
\text { Klinikum Region Hannover Wunstorf }\end{array}$ & $\begin{array}{l}\text { Andreas Spengler } \\
\text { Cornelia Oestereich }\end{array}$ \\
\hline
\end{tabular}




\section{Anhang B: Probandeninformation}

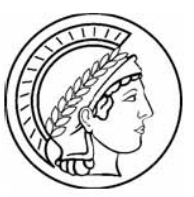

Prof. Dr. Dr. Hannelore Ehrenreich

Division Klinische Neurowissenschaften Max-Planck-Institut für experimentelle Medizin

Hermann-Rein-Strasse 3, D-37075 Göttingen, Tel.: ++49551 3899-628, Fax ++49551 3899-670, Email: ehrenreich@em.mpg.de

\section{Patienteninformation zur Multicenter Schizophrenie-Querschnittserhebung}

Liebe Patientin, lieber Patient,

wir bitten Sie sehr herzlich, an der oben genannten wissenschaftlichen Querschnittserhebung mitzuwirken. Bei dieser Erhebung geht es darum, durch die Mitarbeit vieler an Schizophrenie erkrankter Patienten im Norddeutschen Raum möglichst viel über Ursachen und Hintergründe der Erkrankung "Schizophrenie" zu lernen. Wie Sie vielleicht wissen, betrifft diese Erkrankung immerhin 1-2 Prozent der Bevölkerung, gehört damit also zu den sehr häufigen Erkrankungen des Gehirns. Trotzdem wissen wir über die Krankheit selbst bislang viel zu wenig, um wirklich gut wirksame Behandlungen anbieten zu können.

Im Rahmen der Querschnittserhebung bitten wir Sie, sich insgesamt ca. vier Stunden Zeit zu nehmen. In diesen vier Stunden werden wir eine ausführliche Befragung zu Ihrer Vorgeschichte, zu Begleitkrankheiten und zu Ihrem augenblicklichen sozialen und gesundheitlichen Zustand durchführen. Dazu kommen eine sorgfältige körperliche Untersuchung, eine Hirnleistungstestung (teils mit Papier und Bleistift; teils mittels Computerverfahren), ein Bewegungstest von Händen und Füßen. Schließlich bitten wir Sie um eine Blutentnahme zur Untersuchung auf Gene, die mit der Krankheit Schizophrenie in Verbindung stehen könnten, und auf Antikörper, die Hinweise auf durchgemachte oder bestehende Infektionen geben könnten.

Sollten sich aus diesen Untersuchungen irgendwelche für Sie wichtigen Konsequenzen zeigen, werden Sie selbstverständlich sofort darüber informiert. Die Blutentnahme $(50 \mathrm{ml})$ ist von der Menge her geringfügig (d.h. sie entspricht etwa $10 \%$ einer Blutspende) und harmlos (nur minimales Restrisiko einer Venenentzündung). Alle aus dieser rein wissenschaftlichen Untersuchung erhobenen Daten werden anonymisiert und unterliegen strengen Datenschutzbestimmungen. Wir bitten Sie schon heute vorsorglich um Ihr Einverständnis, dass wir bei wissenschaftlich wichtigen Befunden aus dieser Erhebung mit der Bitte einer weiteren Studienteilnahme auf Sie zukommen dürfen. Die Entscheidung darüber muss dann von Ihnen erneut getroffen werden.

Wir möchten Sie darauf hinweisen, dass Ihre Teilnahme an der Untersuchung freiwillig ist, und dass Sie von der Studienteilnahme keinen direkten Nutzen haben. Sie können jederzeit, auch ohne Angabe von Gründen, Ihre Teilnahme beenden. Dadurch entstehen Ihnen keinerlei Nachteile. Lassen Sie sich ausreichend Zeit für Ihre Entscheidung zur Teilnahme an dieser Untersuchung. Bitte stellen Sie Ihrem Arzt alle Fragen, die für Ihr Verständnis wichtig sind.

Leiterin der klinischen Untersuchung:

Prof. Dr. Dr. Hannelore Ehrenreich,

Max-Planck-Institut für experimentelle Medizin

Hermann-Rein-Str. 3

D-37075 Göttingen 


\section{Anhang C: Übersicht über die in der GRAS-Studie verwendeten psy- chometrischen, neuropsychologischen und neurologischen Messin- strumente.}

\begin{tabular}{|c|c|c|c|}
\hline \multicolumn{4}{|c|}{ Psychometrische Beurteilungsskalen und Fragebögen } \\
\hline BSI & \multicolumn{2}{|c|}{ Brief Symptom Inventory (deutsche Version) } & (Franke 2000) \\
\hline CGI & \multicolumn{2}{|c|}{ Clinical Global Impressions Scale (deutsche Version) } & (Guy 1976) \\
\hline GAF & \multicolumn{2}{|c|}{ Global Assessment of Functioning Scale (deutsche Version) } & $\begin{array}{l}\text { (AmericanPsychiatricAssociation } \\
2000)\end{array}$ \\
\hline PANSS & \multicolumn{2}{|c|}{ Positive and negative Syndrome Scale (deutsche Version) } & (Kay et al. 1987) \\
\hline STAI & \multicolumn{2}{|c|}{ State-Trait-Angstinventar } & (Laux et al. 1981) \\
\hline TAS-26 & \multicolumn{2}{|c|}{ Toronto-Alexithymie-Skala-26 } & (Kupfer et al. 2001) \\
\hline \multicolumn{4}{|c|}{ Neuropsychologische Tests } \\
\hline & $\begin{array}{l}\text { Testbatterie zur Aufmerksam- } \\
\text { keitsprüfung: Subtests ,Alert- } \\
\text { ness' und 'geteilte Aufmerk- } \\
\text { samkeit' }\end{array}$ & $\begin{array}{l}\text { Alertness und geteilte Auf- } \\
\text { merksamkeit }\end{array}$ & (Zimmermann und Fimm 1993) \\
\hline \multirow[t]{2}{*}{ BZT } & $\begin{array}{l}\text { Wechsler Intelligenztest für } \\
\text { Erwachsene (WIE): Buchsta- } \\
\text { ben-Zahlen-Test }\end{array}$ & Arbeitsgedächtnis & (Wechsler 1998) \\
\hline & Dotting & $\begin{array}{l}\text { Feinmotorische Geschwin- } \\
\text { digkeit und Koordination }\end{array}$ & (MacQuarrie 1925, 1953) \\
\hline LPS-3 & Leistungsprüfsystem: Subtest 3 & Schlussfolgenderes Denken & (Horn 1983) \\
\hline \multirow[t]{2}{*}{ MWT-B } & $\begin{array}{l}\text { Mehrfach-Wortschatz- } \\
\text { Intelligenztest Version B }\end{array}$ & $\begin{array}{l}\text { Prämorbide bzw. Kristalli- } \\
\text { sierte Intelligenz }\end{array}$ & (Lehrl 1999) \\
\hline & Tapping & $\begin{array}{l}\text { Feinmotorische Geschwin- } \\
\text { digkeit und Koordination }\end{array}$ & (MacQuarrie 1925, 1953) \\
\hline TMT-A & Trail Making Test A & Verarbeitungsgeschwindigkeit & (Reitan 1958) \\
\hline \multirow[t]{2}{*}{ TMT-B } & Trail Making Test B & Exekutivfunktionen & (Reitan 1958) \\
\hline & $\begin{array}{l}\text { Regensburger Wortflüssigkeits- } \\
\text { Test }\end{array}$ & Verbale Flüssigkeit & (Aschenbrenner et al. 2000) \\
\hline VLMT & $\begin{array}{l}\text { Verbaler Lern-und Merkfähig- } \\
\text { keitstest }\end{array}$ & $\begin{array}{l}\text { Verbales Lernen und Ge- } \\
\text { dächtnis }\end{array}$ & (Helmstädter et al. 2001) \\
\hline ZST & $\begin{array}{l}\text { Hamburg-Wechsler Intelligenz- } \\
\text { test für Erwachsene (HAWIE- } \\
\text { R): Zahlen-Symbol-Test }\end{array}$ & $\begin{array}{l}\text { Psychomotorische Ge- } \\
\text { schwindigkeit, Konzentration } \\
\text { und Aufmerksamkeit }\end{array}$ & (Tewes 1991) \\
\hline \multicolumn{4}{|c|}{ Neurologische Tests } \\
\hline AIMS & \multicolumn{2}{|c|}{ Abnormal Involuntary Movement Scale } & (Guy 1976) \\
\hline BAS & \multicolumn{2}{|c|}{ Barnes-Akathisa Scale } & (Barnes 1989) \\
\hline CNI & \multicolumn{2}{|l|}{ Cambridge Neurological Inventory } & (Chen et al. 1995) \\
\hline \multirow[t]{2}{*}{ COMO } & \multicolumn{2}{|l|}{ Contralateral Co-Movement Task } & (Bartels C et al. 2008) \\
\hline & \multicolumn{2}{|l|}{ Geruchstest } & $\begin{array}{l}\text { Nutzung kommerziell verfügbarer } \\
\text { Geruchsproben } \\
\text { (www.sensonics.com) (a) zur } \\
\text { Geruchserkennung in einem Multiple } \\
\text { Choice Setting (b) zur Benennung und } \\
\text { Eigenschaftszuweisung von Gerüchen. }\end{array}$ \\
\hline SAS & \multicolumn{2}{|l|}{ Simpson-Angus Scale } & (Simpson und Angus 1970) \\
\hline TDRS & \multicolumn{2}{|l|}{ Tardive Dyskinesia Rating Scale } & (Simpson et al. 1979) \\
\hline
\end{tabular}




\section{Anhang D: Kategorieneinteilung der von Patienten angegebenen Ne- benwirkungssymptome}

\begin{tabular}{|c|c|c|}
\hline Kategorie & Mechanismus & Symptomatik \\
\hline neurologisch & motorisch & $\begin{array}{l}\text { Dyskinesie / Dystonie: } \\
\text { Torticollis, Retrocollis, Torsionsdystonie, okulogyre Krisen, Zungen- } \\
\text { Schlundkrämpfe } \\
\text { Parkinsonoid: Rigor, Tremor, Hypokinese } \\
\text { Akathisie: Bewegungsunruhe }\end{array}$ \\
\hline \multirow{6}{*}{$\begin{array}{l}\text { metabolisch- } \\
\text { endokrin }\end{array}$} & antidopaminerg & $\begin{array}{l}\text { Hyperprolaktinämie: Menorrhoe, Galaktorrhoe, Gynäkomastie, Veränderungen der } \\
\text { Libido, Priapismus, Anorgasmie }\end{array}$ \\
\hline & & Metabolisches Syndrom \\
\hline & & Gewichtszunahme \\
\hline & & Diabetes mellitus \\
\hline & anticholinerg & Bauchschmerzen, Obstipation, Mundtrockenheit, Sehstörungen, Miktionsstörungen \\
\hline & cholinerg & Hyperhidrosis \\
\hline \multirow{5}{*}{ kardiovaskulär } & $\alpha$-adrenerg & Orthostatische Dysregulation, Schwindel \\
\hline & & arterielle Hypertonie \\
\hline & & Herzrhythmusstörungen \\
\hline & & koronare Herzkrankheit \\
\hline & & Thrombose / Embolie \\
\hline psychiatrisch & & Sedierung, Konzentrationsschwierigkeiten, affektive Schwankungen \\
\hline
\end{tabular}

\section{Anhang E: Tabelle der in Depotform applizierten AP mit verwendeten Umrechnungsfaktoren}

\begin{tabular}{|l|l|c|c|c|l|}
\hline \multicolumn{1}{|c|}{ Wirkstoff } & Handelsname & $\begin{array}{c}\text { Ty- } \\
\text { pus }\end{array}$ & $\begin{array}{c}\text { Chlorpromazin- } \\
\text { koeffizient (CPÄ/mg) }\end{array}$ & $\begin{array}{c}\text { CPÄ- } \\
\text { Bestimmung }\end{array}$ & Quelle \\
\hline Bromperidoldecanoat & Impromen & F & 100 & A & (McLaren et al. 1992) \\
\hline Clopenthixoldecanoate & Ciatyl-Depot & F & 31,5 & A & (Müller P 1987) \\
\hline Droperidol-injection & Inapsine & F & 100 & A & (Hyman et al. 1995) \\
\hline Flupenthixol Decanoate & Fluanxol depot & F & 100 & B & (Schulz et al. 1989) \\
\hline Fluphenazindecanoat & Lyogen Depot & F & 200 & B & (Schulz et al. 1989) \\
\hline Fluspirilen & Imap & F & 525 & C & (Müller P 1999) \\
\hline Haloperidoldecanoat & Haldol Decanoat & F & 70 & B & (Schulz et al. 1989) \\
\hline Perphenazinoenanthat & Decentan Depot & F & 46 & B & (Schulz et al. 1989) \\
\hline Pipothiazine palmitate & & F & 100 & D & (Kane et al. 1998) \\
\hline Resperidon-Depot & Risperdal-Consta & S & 84 & E & (Eerdekens et al. 2004) \\
\hline Zuclopenthixolacetat & Ciatyl-z-Acuphase & F & 18 & C & (Müller P 1999) \\
\hline Zuclopenthixoldecanoat & Ciatyl-z-Depot & F & 16 & B & (Schulz et al. 1989) \\
\hline
\end{tabular}

Bei den als Depot verabreichten Wirkstoffen ist die Tabelle wie folgt zu verstehen: Umrechnungsfaktor $(\mathrm{CPZÄ} / \mathrm{mg} / \mathrm{d}) *$ Dosis $(\mathrm{mg}) /$ Tage des Depotintervalls (d) ergibt die antipsychotische Tagesdosis. F: FGA/ Typikum, S: SGA/ Atypikum

A: Klinische Erfahrungswerte, B: Vergleich von Bioverfügbarkeit, molekularem Gewicht, Applikationsintervall,

C: Wirksamkeit in klinischen Studien; D: Erschließung aus klinischen Studien, E: Vergleich von Plasmaleveln 


\section{Anhang F: Tabelle oral applizierter AP mit verwendeten \\ Umrechnungsfaktoren}

\begin{tabular}{|c|c|c|c|c|c|}
\hline Wirkstoff & Handelsname & $\begin{array}{l}\text { Ty- } \\
\text { pus }\end{array}$ & $\begin{array}{c}\text { Chlorpromazin- } \\
\text { koeffizient (CPÄ/mg) }\end{array}$ & $\begin{array}{l}\mathrm{CPÄ-Be}- \\
\text { stimmung }\end{array}$ & Quellenangabe \\
\hline Acetophenazin & Tindal & $\mathrm{F}$ & 5 & $\mathrm{~A}$ & (Rey et al. 1989) \\
\hline Amisulprid & Solian & $\mathrm{S}$ & 0,2 & $\mathrm{~B}$ & (Müller WE 2001) \\
\hline Aripiprazol & Abilify & $\mathrm{S}$ & 13,33 & $\mathrm{C}$ & (Woods 2003) \\
\hline Benperidol & Glianimon & $\mathrm{F}$ & 75 & $\mathrm{~B}$ & (Müller WE 2001) \\
\hline Bromperidol & Impromen & $\mathrm{F}$ & 50 & $\mathrm{~B}$ & (Müller P 1999) \\
\hline Butaperazin & Repoise & $\mathrm{F}$ & 12,5 & A & (Rey et al. 1989) \\
\hline Carphenazine & Proketazine & $\mathrm{F}$ & 4 & A & (Rey et al. 1989) \\
\hline Chlopenthixol & Ciatyl & $\mathrm{F}$ & 2,5 & $\mathrm{D}$ & (Haase 1969) \\
\hline Chlorprothixen & \begin{tabular}{|l|} 
Truxal \\
\end{tabular} & $\mathrm{F}$ & 1,5 & $\mathrm{~A}$ & (Rey et al. 1989) \\
\hline Clozapin & \begin{tabular}{|l|} 
Leponex \\
\end{tabular} & $\mathrm{S}$ & 1,5 & $\mathrm{~A}$ & (Rey et al. 1989) \\
\hline Droperidol & Inapsine & $\mathrm{F}$ & 10 & $\mathrm{E}$ & (Kaplan und Sadock 1993) \\
\hline Flupentixol & Fluanxol & $\mathrm{F}$ & 50 & $\mathrm{D}$ & (Haase 1969) \\
\hline Fluphenazin & \begin{tabular}{|l|} 
Dapotum \\
\end{tabular} & $\mathrm{F}$ & 40 & $\mathrm{~B}$ & (Müller P 1999) \\
\hline Fluphenazin & \begin{tabular}{|l|} 
Lyogen \\
\end{tabular} & $\mathrm{F}$ & 40 & $\mathrm{~B}$ & (Müller P 1999) \\
\hline Haloperidol & Haldol & $\mathrm{F}$ & 50 & $\mathrm{D}$ & (Haase 1969) \\
\hline Levomepromazin & Neurocil & $\mathrm{F}$ & 0,8 & $\mathrm{~B}$ & (Müller P 1999) \\
\hline Loxapine & Loxitane & $\mathrm{F}$ & 10 & A & (Rey et al. 1989) \\
\hline Melperon & \begin{tabular}{|l|} 
Eunerpan \\
\end{tabular} & $\mathrm{F}$ & 1 & $\mathrm{~B}$ & (Müller WE 2001) \\
\hline Mesoridazine & Serentil & $\mathrm{F}$ & 2 & $\mathrm{~B}$ & (Kane et al. 1998) \\
\hline Methophenazin & Frenolon & $\mathrm{F}$ & 4,5 & $\mathrm{~F}$ & (Borsy et al. 1965) \\
\hline Molindone & \begin{tabular}{|l|} 
Lidone \\
\end{tabular} & $\mathrm{F}$ & 10 & $E$ & (AmericanPsychiatricAssociation 1997) \\
\hline Molindone & Moban & $\mathrm{F}$ & 10 & $\mathrm{E}$ & (AmericanPsychiatricAssociation 1997) \\
\hline Olanzapin & Zyprexa & $\mathrm{S}$ & 40 & $\mathrm{E}$ & (AmericanPsychiatricAssociation 1997) \\
\hline Paliperidon & \begin{tabular}{|l|} 
Invega \\
\end{tabular} & $\mathrm{S}$ & 45 & G & (Schneider 2010) \\
\hline Perazin & \begin{tabular}{|l|} 
Taxilan \\
\end{tabular} & $\mathrm{F}$ & 0,7 & $\mathrm{~B}$ & (Müller P 1999) \\
\hline Pericyazine & Neuleptil & $\mathrm{F}$ & 7,5 & $\mathrm{~A}$ & (Rey et al. 1989) \\
\hline Perphenazin & Decentan & $\mathrm{F}$ & 10 & $\mathrm{D}$ & (Haase 1969) \\
\hline Pimozide & Orap & $\mathrm{F}$ & 75 & $\mathrm{~A}$ & (Rey et al. 1989) \\
\hline Pipamperon & Dipiperon & $\mathrm{F}$ & 0,7 & $\mathrm{~B}$ & (Müller P 1999) \\
\hline Piperacetazine & Quide & $\mathrm{F}$ & 12,5 & $\mathrm{~A}$ & (Rey et al. 1989) \\
\hline Prochlorperazine & Compazine & $\mathrm{F}$ & 5,5 & $\mathrm{~A}$ & (Rey et al. 1989) \\
\hline Promazin & Sparine & $\mathrm{F}$ & 0,5 & $\mathrm{~A}$ & (Rey et al. 1989) \\
\hline Promethazin & Atosil & $\mathrm{F}$ & 0,7 & $\mathrm{E}$ & (Ehrenreich 2010) \\
\hline Prothipendyl & Dominal & $\mathrm{F}$ & 0,7 & $\mathrm{~B}$ & (Müller WE 2001) \\
\hline Quetiapin & \begin{tabular}{|l|} 
Seroquel \\
\end{tabular} & $\mathrm{S}$ & 1 & $\mathrm{~B}$ & (Müller P 1999) \\
\hline Risperdon & Risperdal & $\mathrm{S}$ & 75 & $\mathrm{H}$ & (Davis und Chen 2004) \\
\hline Sulpiride & Dogmatil & $\mathrm{S}$ & 0,5 & $\mathrm{~B}$ & (Müller P 1999) \\
\hline Thioridazin & Mellaril & $\mathrm{F}$ & 1 & $\mathrm{~A}$ & (Rey et al. 1989) \\
\hline Thiothixen & Navane & $\mathrm{F}$ & 20 & $\mathrm{D}$ & (Haase 1969) \\
\hline Thiothixen & Orbinamon & $\mathrm{F}$ & 20 & $\mathrm{D}$ & (Haase 1969) \\
\hline Tiapride & Tiaprid & $\mathrm{F}$ & 1 & $\mathrm{~A}$ & (Rey et al. 1989) \\
\hline Trifluoperazin & Stelazin & $\mathrm{F}$ & 20 & $\mathrm{D}$ & (Haase 1969) \\
\hline Trifluperazine & Eskazinyl & $\mathrm{F}$ & 20 & A & (Rey et al. 1989) \\
\hline Trifluperidol & Triperidol & $\mathrm{F}$ & 100 & $\mathrm{~B}$ & (Müller P 1999) \\
\hline Triflupromazine & \begin{tabular}{|l|} 
Vesprin \\
\end{tabular} & $\mathrm{F}$ & 3 & $\mathrm{D}$ & (Haase 1969) \\
\hline Ziprasidone & \begin{tabular}{|l|} 
Zeldox \\
\end{tabular} & $\mathrm{S}$ & 1,667 & $\mathrm{C}$ & (Woods 2003) \\
\hline Zotepine & Nipolept & $\mathrm{S}$ & 2 & $\mathrm{~B}$ & (Müller P 1999) \\
\hline Zuclopenthixol & Ciatyl-Z & $\mathrm{F}$ & 5 & $\mathrm{~A}$ & (Rey et al. 1989) \\
\hline
\end{tabular}

Nach dem Wirkstoff und dem entsprechendem Handelsnamen folgt die Spalte mit dem Medikamententypus (F: FGA/Typikum; S: SGA/Atypikum), darauf folgt der Umrechnungsfaktor und die Quellenangabe. Umrechnungsfaktor $(\mathrm{CP} \ddot{A} / \mathrm{mg}) *$ Dosis $(\mathrm{mg})$ des Wirkstoffes ergibt das entsprechende CPÄ. Bei Probanden, die mehrere antipsychotische Präperate einnahmen, wurden die einzelnen CPÄ addiert.

A: Vergleich klinischer und experimenteller Werte, B: klinische Studien, C: Vergleich minimaler Effektdosen, D: Vergleich von Schwellendosen durch EPMS, E: klinische Erfahrungswerte, F: Dosisvergleich im Tierexperiment, G: Vergleich mittlerer antipsychotischer Tagesdosen, H: Vergleich der Halbmaximaldosen und klinischer Wirksamkeit 


\section{Anhang G: Häufigkeitsverteilung des Alters zum Zeitpunkt der GRAS-}

\section{Untersuchung}

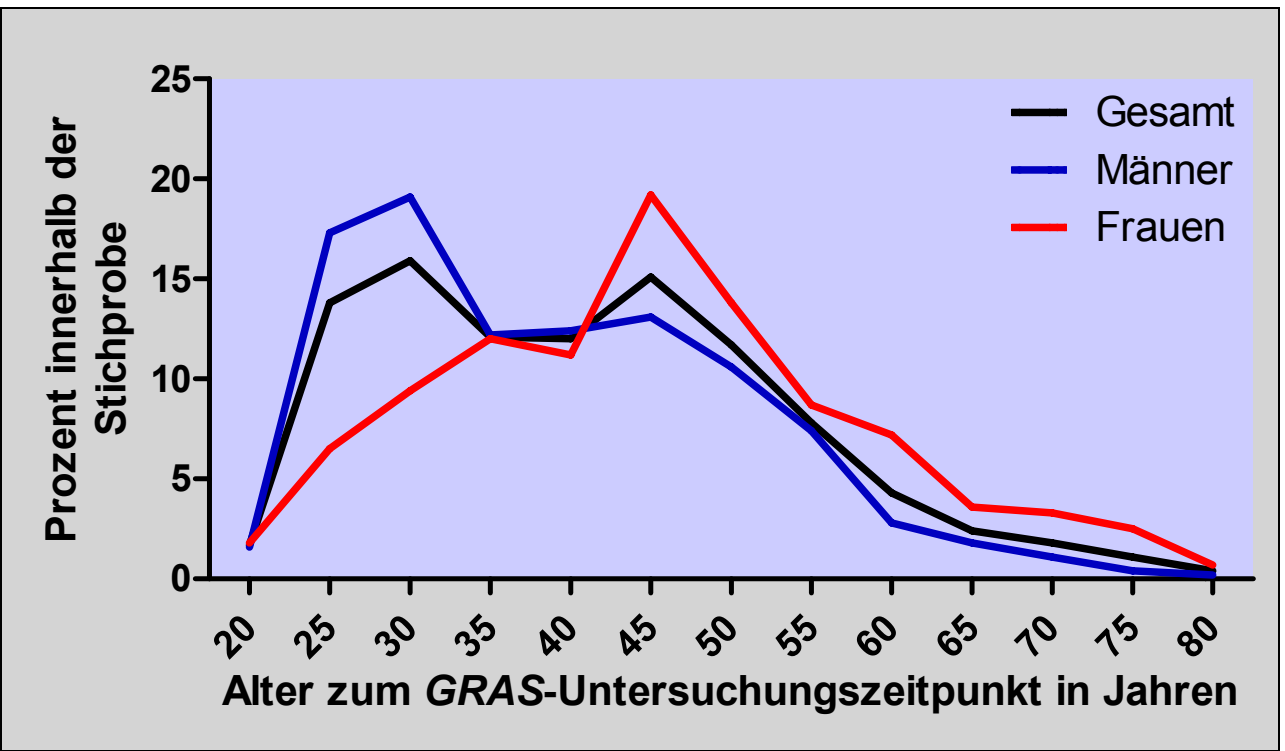

Die Angaben basieren auf den Untersuchungen von 753 Probanden, von denen die exakte antipsychotische Dosissteigerung pro Jahr bekannt war, aufgeteilt in 512 Männer und 241 Frauen.

\section{Anhang H Untersuchungsmaterialien}

\section{Anhang H1: Neuropsychologische Inventare}
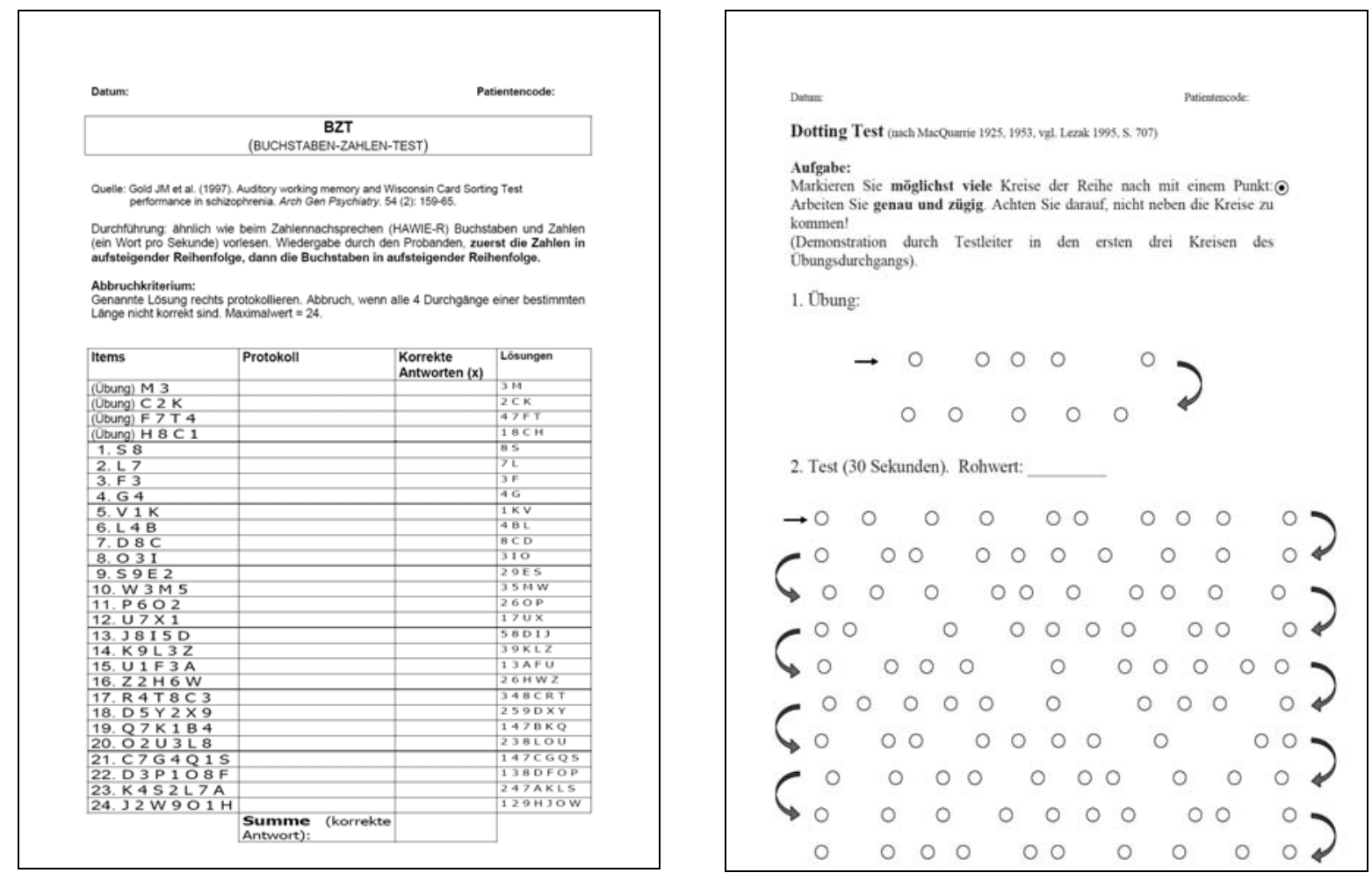

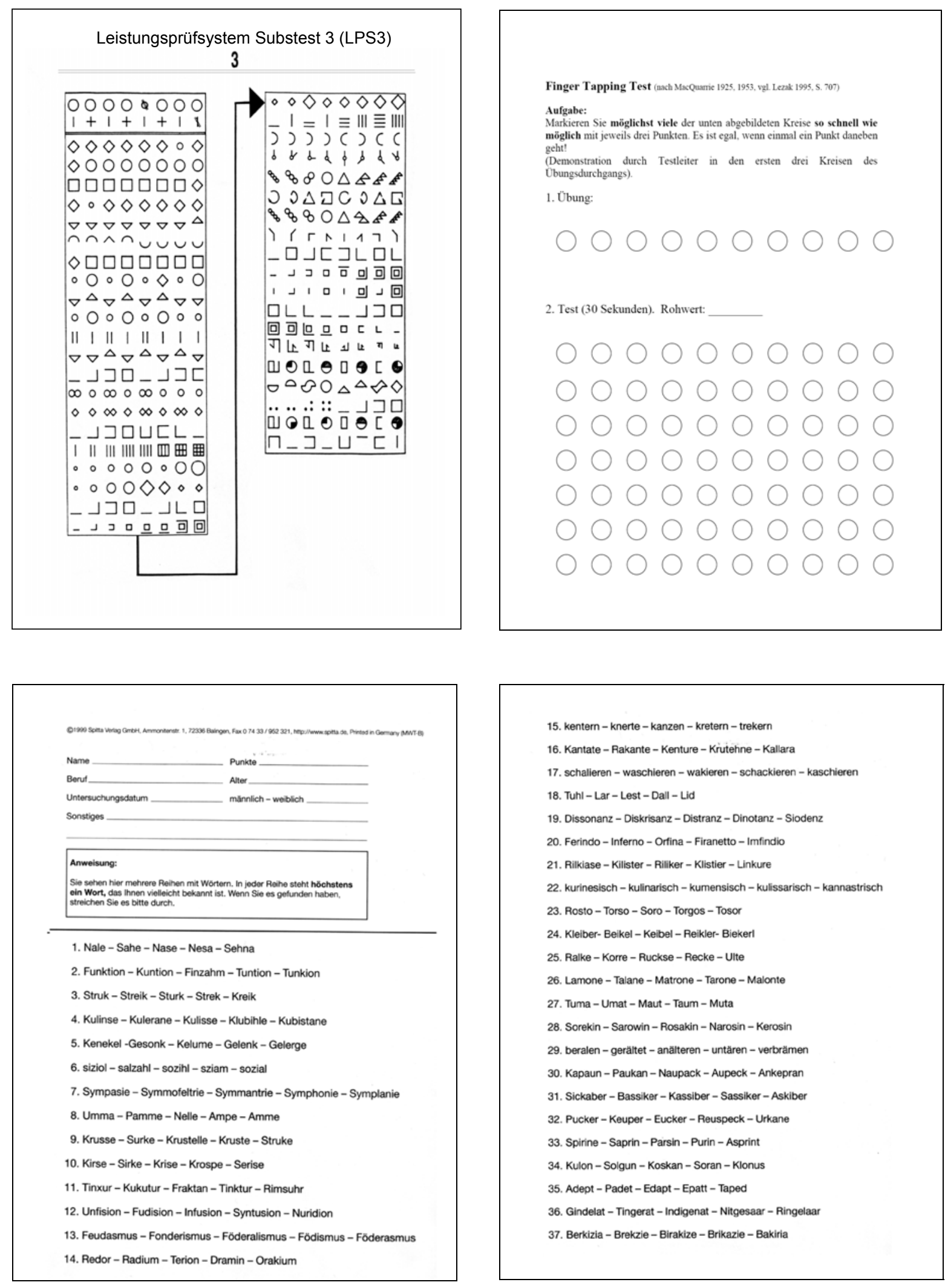

15. kentern - knerte - kanzen - kretern - trekern

16. Kantate - Rakante - Kenture - Krutehne - Kallara

17. schalieren - waschieren - wakieren - schackieren - kaschieren

18. Tuhl - Lar - Lest - Dall - Lid

19. Dissonanz - Diskrisanz - Distranz - Dinotanz - Siodenz

20. Ferindo - Inferno - Orfina - Firanetto - Imfindio

21. Rilkiase - Killister - Rilliker - Klistier - Linkure

22. kurinesisch - kulinarisch - kumensisch - kulissarisch - kannastrisch

23. Rosto - Torso - Soro - Torgos - Tosor

24. Kleiber- Belkel - Keibel - Reikler-Biekerl

25. Ralke - Korre - Ruckse - Recke - Ulte

26. Lamone - Talane - Matrone - Tarone - Malonte

27. Tuma - Umat - Maut - Taum - Muta

28. Sorekin - Sarowin - Rosakin - Narosin - Kerosin

29. beralen - geraltet - anălteren - untären - verbrämen

30. Kapaun - Paukan - Naupack - Aupeck - Ankepran

31. Sickaber - Bassiker - Kassiber - Sassiker - Askiber

32. Pucker - Keuper - Eucker - Reuspeck - Urkane

33. Spirine - Saprin - Parsin - Purin - Asprint

34. Kulon - Solgun - Koskan - Soran - Klonus

35. Adept - Padet - Edapt - Epatt - Taped

36. Gindelat - Tingerat - Indigenat - Nitgesaar - Ringelaar

37. Berkizia - Brekzie - Birakize - Brikazie - Bakiria 

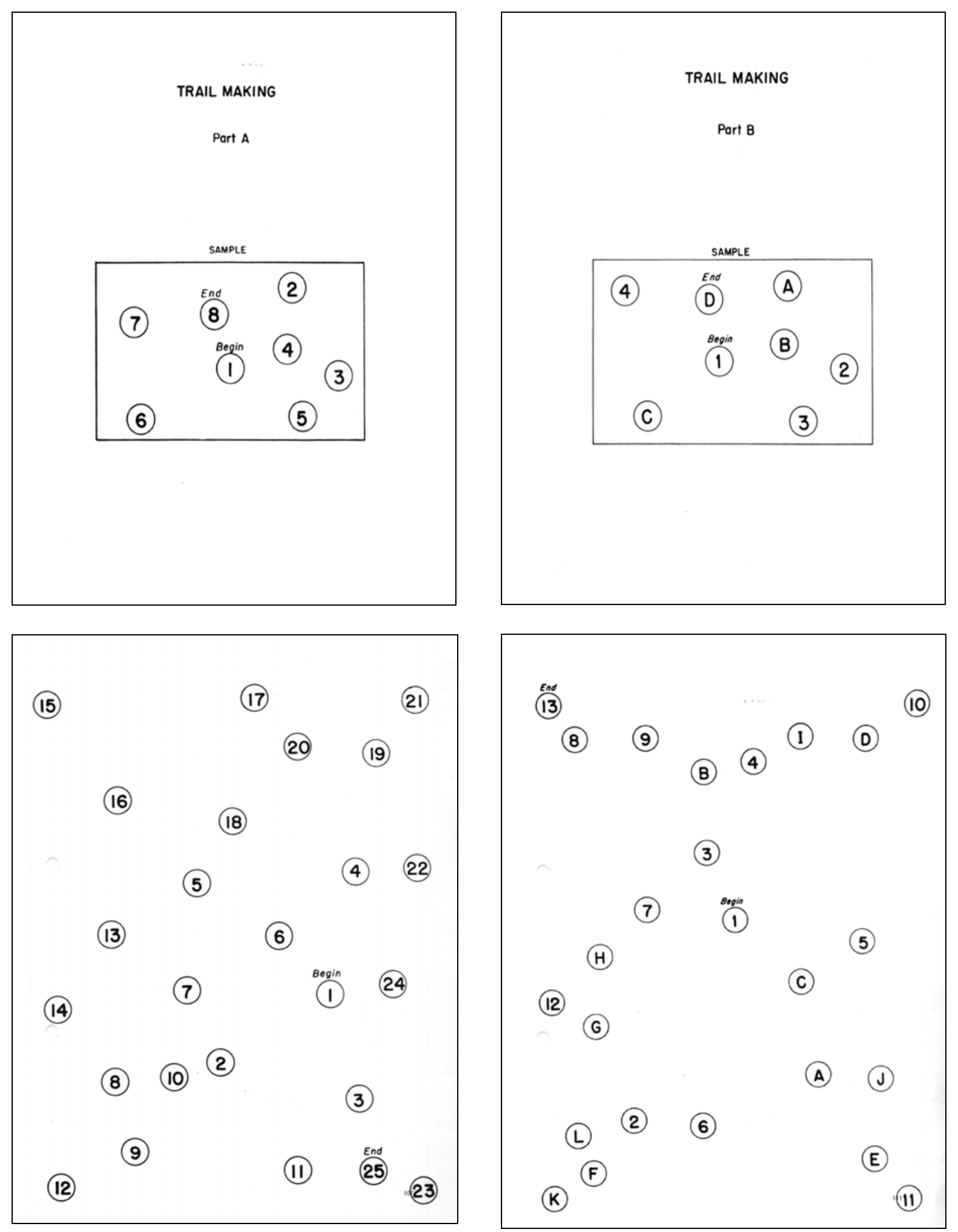

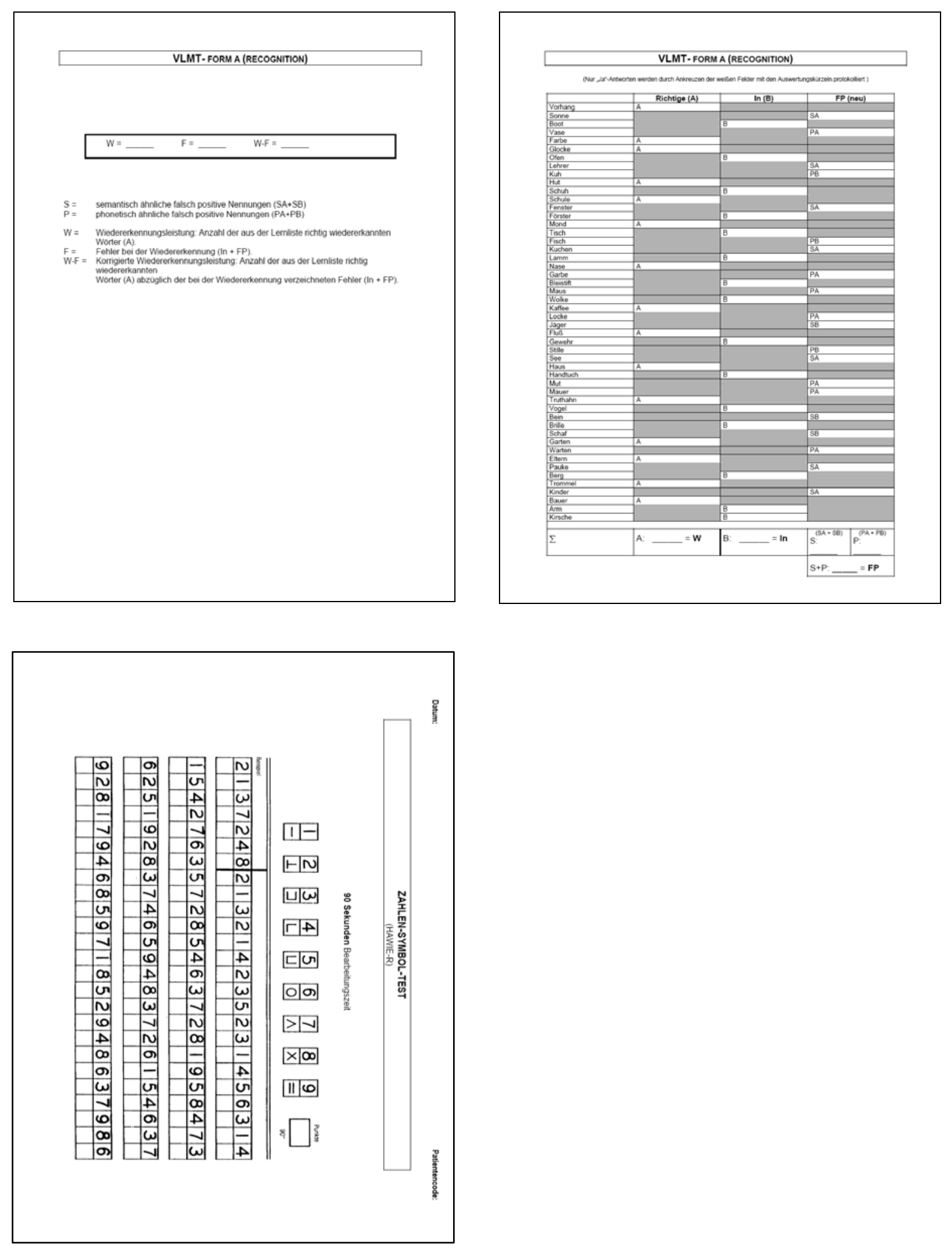
Anhang H2: Skala zur Enschätzung der Psychopathologie
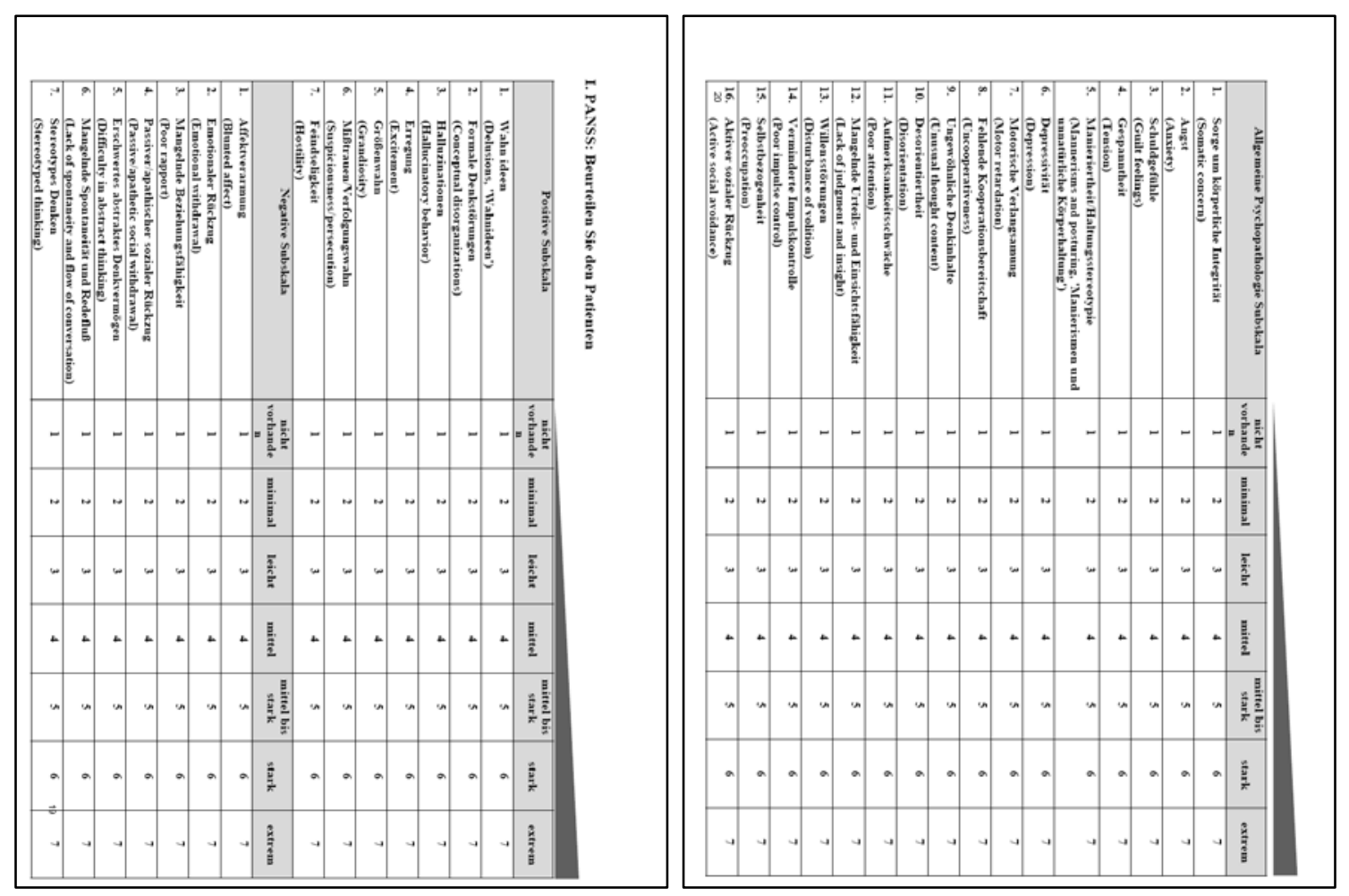

Anhang H3: Skalen zur Einschätzung der Erkrankungsschwere
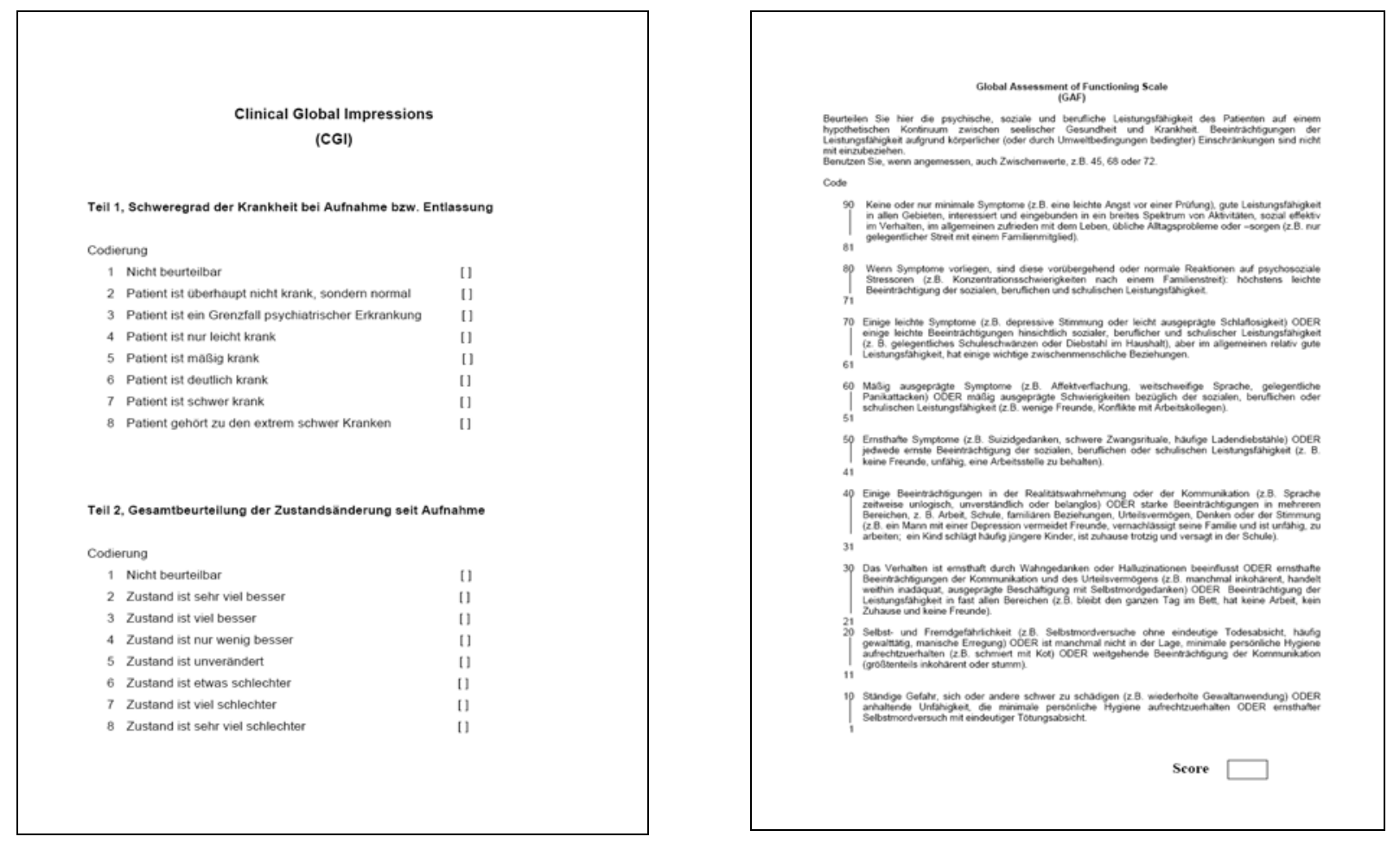


\section{Anhang H4: Inventar zur Untersuchung der neurologischen Erkran-}

\section{kungsschwere}
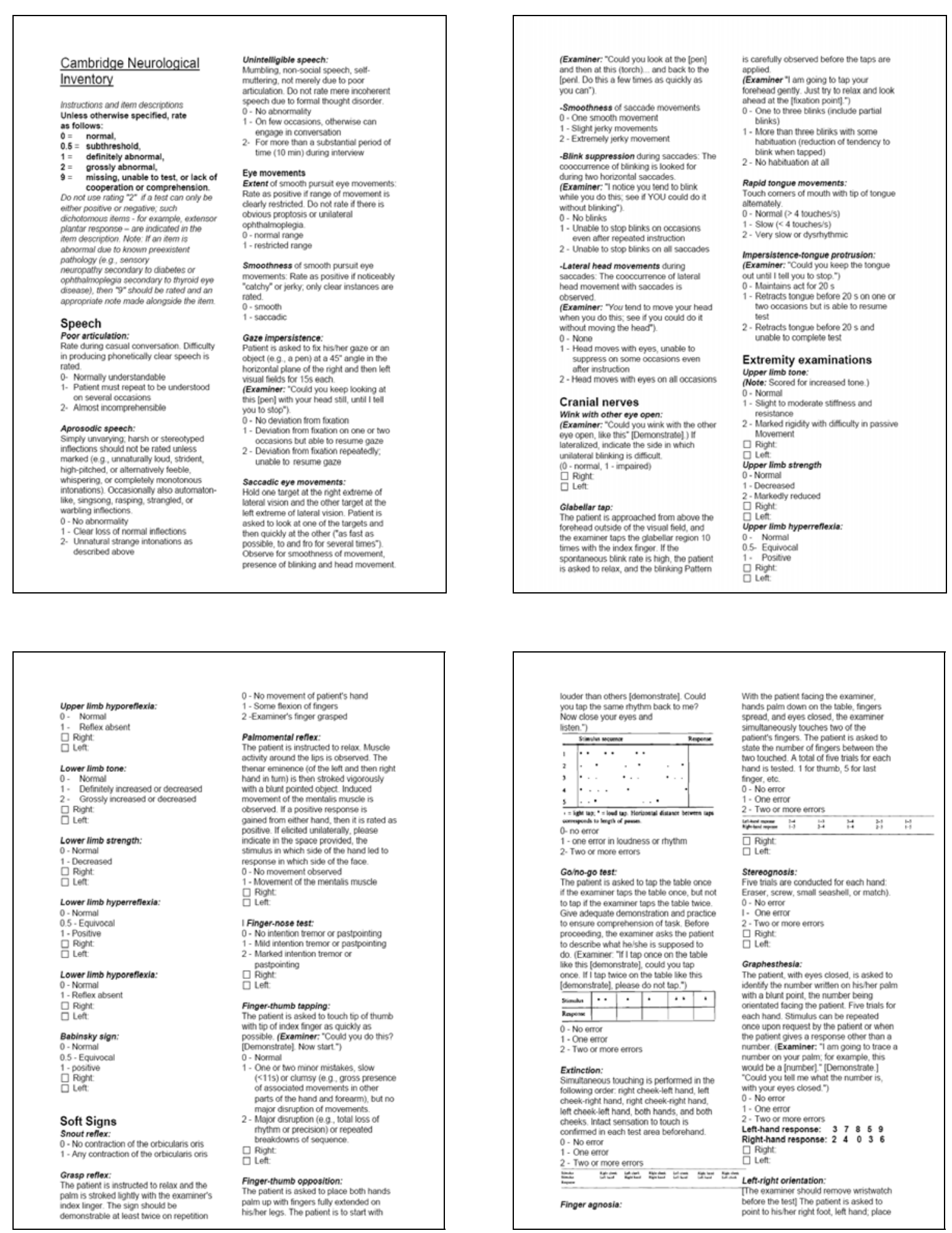


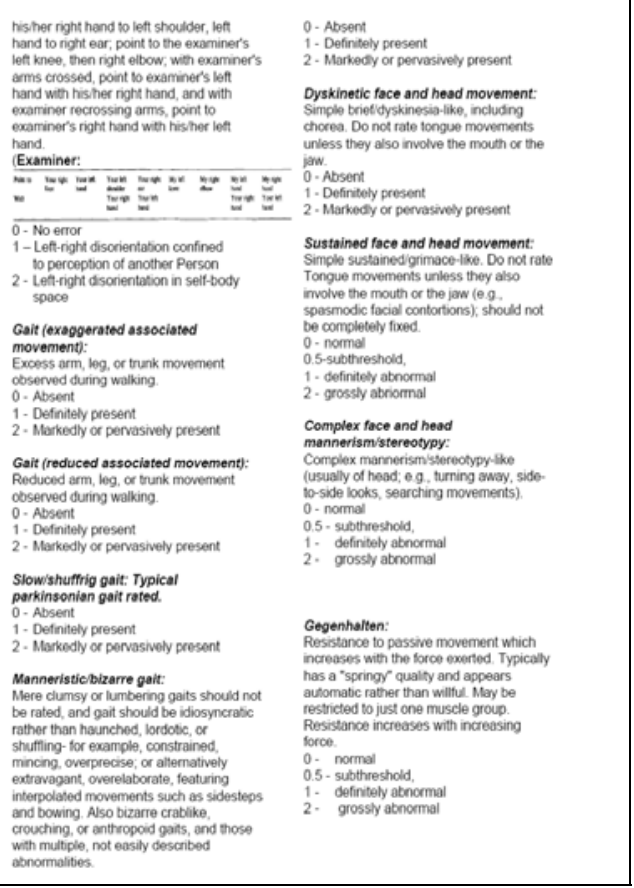

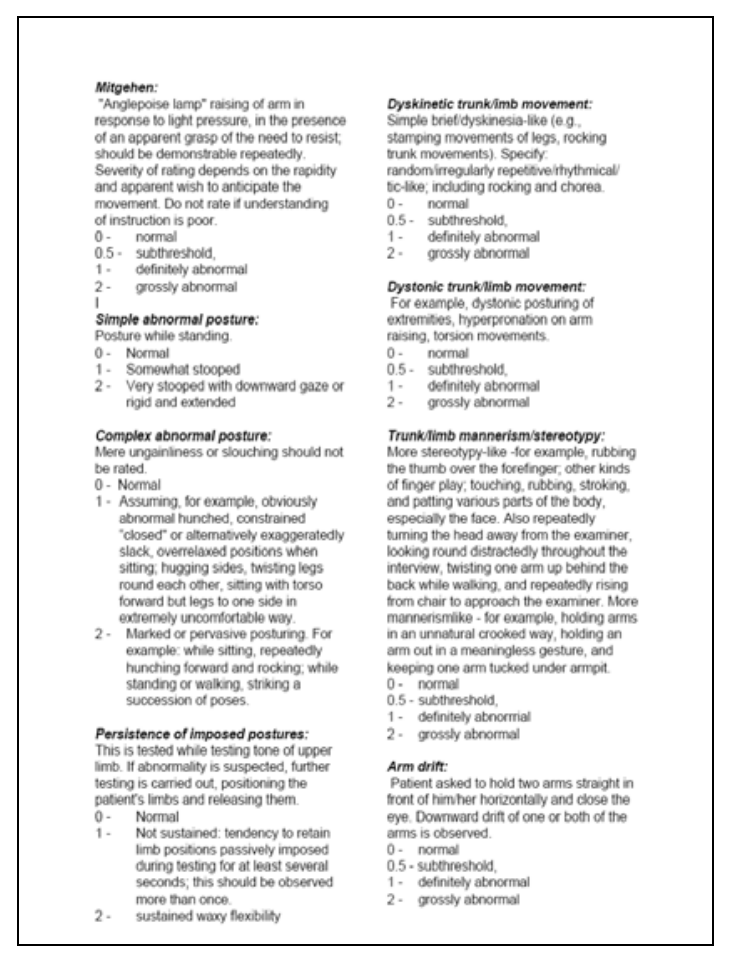

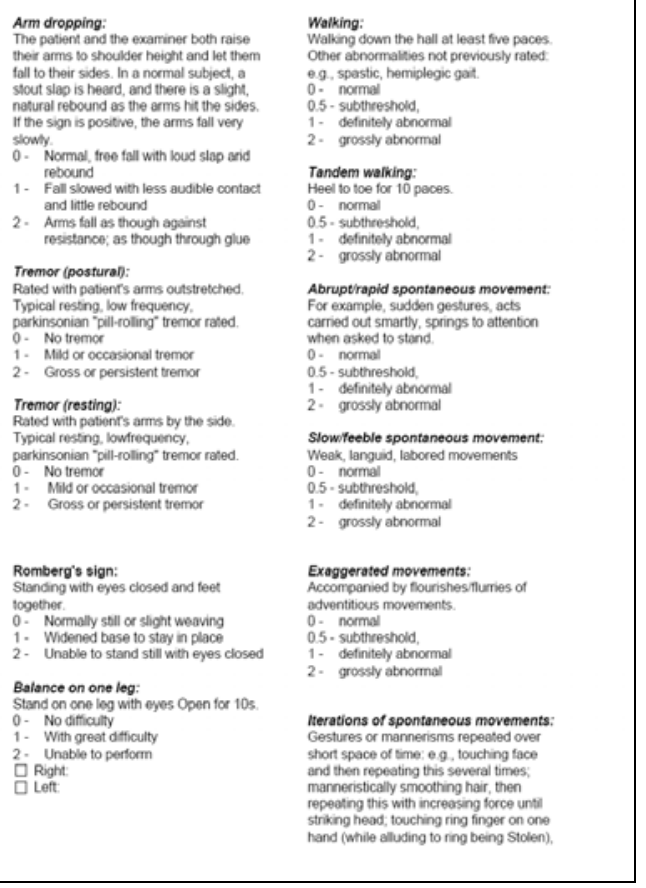

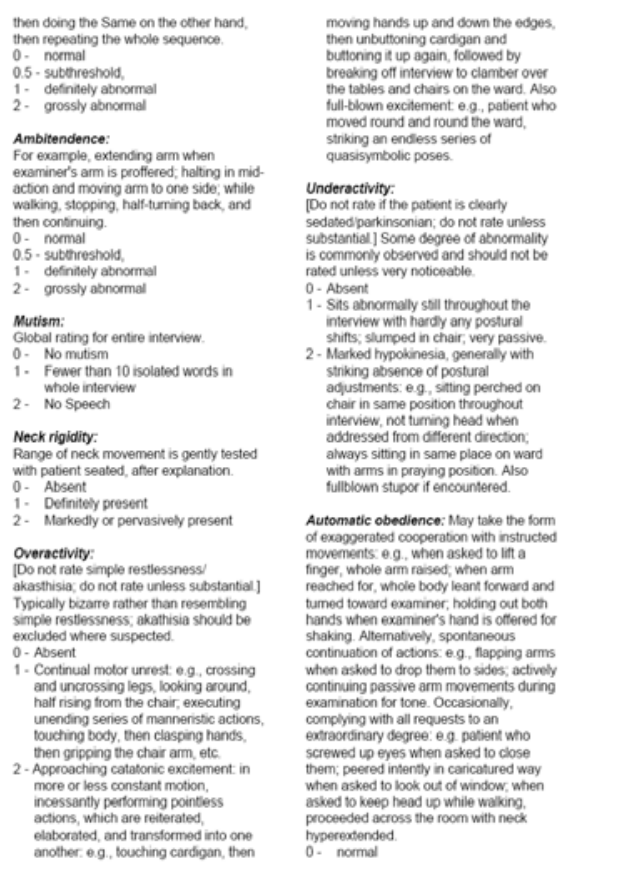




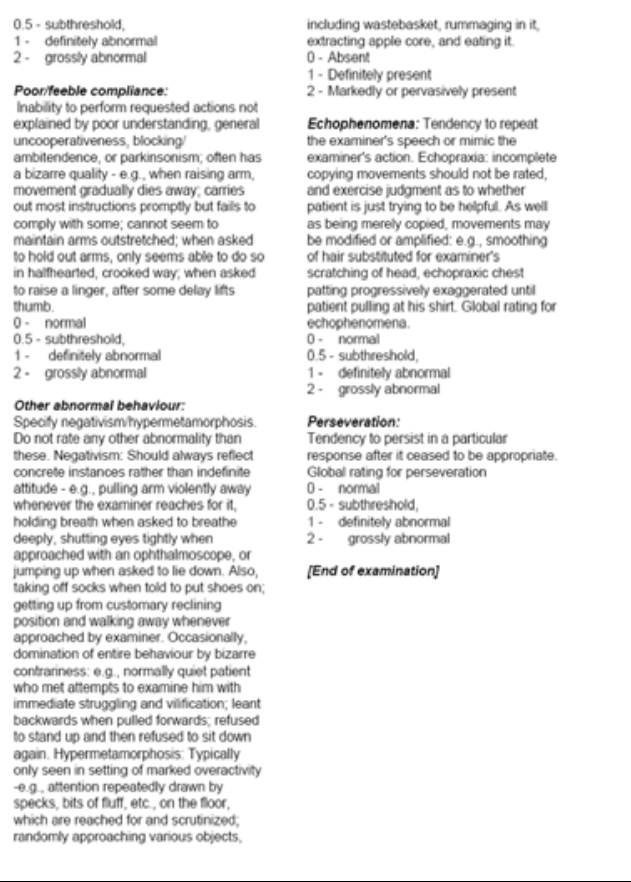

\section{Anhang H5: Inventare zur Untersuchung der Nebenwirkungen antipsy- chotischer Therapie}

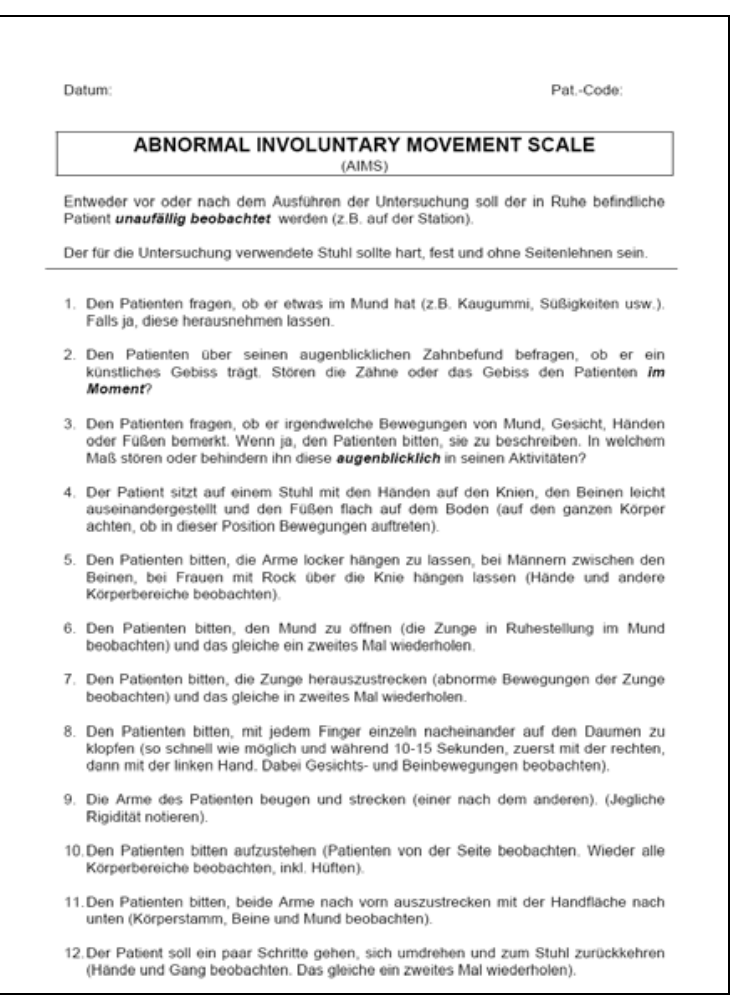

Bitte beachten Sie fur die Ausfuhrung und Bewertung die englische EXAMINATION copar

Kodierung:
$0=$ keine, minim
$1=$ minit,

$0=$ keine, minimal
$1=$ minimal, Normgrenze
$2=$ leicht
$3=$

$2=$ leicht
$3=$ mittel

$4=$ schwer
Bewertungen der Bewegungen oder Bewegungsunruhe:

Immer hochsten beobachteten Schweregrad angeben

Gesicht und Mund

1. Muskeln für den Gesichtsausdruck
2B. Bowegungen von Stim, Augonbraven, periorbitaler Beroich, Wangen

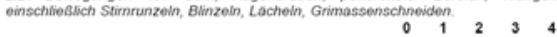

2. Lippen und perioraler Bereich

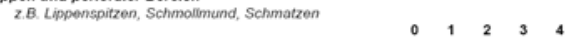

3. Kieferbereich

ZB. Beisen, Zahne zusammenpressen, Kauen, Mund otthen, seitiche
Kifferbewegungen

Zunge

$\begin{array}{lllll}0 & 1 & 2 & 3 & 4\end{array}$

Nur aberschiossende und zusatziliche Bewegungen in oder auserhall des Mundes

Extremitäten

5. Obere Extremitaten

(Arme, Handgelenke, Hände, Finger)

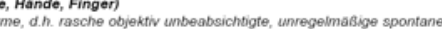

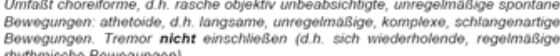
hythmische Bewegungen)

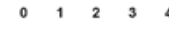

6. Untere Extremitäten
(Beine, Knie, Knóchel, Zehen)

ZB. seitiche Kniebewegungen, Klopten mit den Falen, mit den Fersen auf den Per Patient soll ein paor Schrite gehen, sich undreben und zum Stuhl zurockkehren

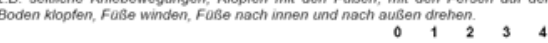




\section{Körperstamm}

7. Genick, Schultern, Becken

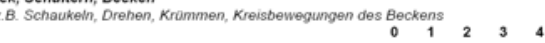

\section{Globale Beurteilung}

8. Schweregrad der abnormen Bewegungen

9. Behinderung durch abnorme Bewegungen

$\begin{array}{llll}1 & 2 & 3 & 4\end{array}$

(nur Angaben des Patientent verwerten)

nicht bewusst

bewusst / nicht qualend
bewusst / leicht qualend

3 bewusst / mittelgradig qualt

\section{Zahnbefund}

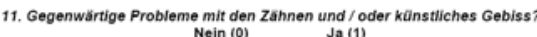

12. Trägt der Patient in der Regel ein künstliches Gebiss?
Nein (0) Ja (1)

Bitte addieron Sie die angekreuzten Abstufungen der Items 1-7 zu einem
Summenscore. Summenscore.

SUMMENSCORE:

\begin{tabular}{|c|c|}
\hline 22. Schaskeltbewogungen ma dem Obenkopper & 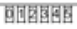 \\
\hline 23. Sonstige: & 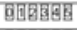 \\
\hline 24. Sonstige: & 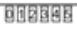 \\
\hline \multicolumn{2}{|l|}{ Obere Extremitsten } \\
\hline 25. Ballistische Bewegungen & 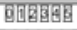 \\
\hline 26. Chores-atheloide Bewegungen der Finger & 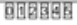 \\
\hline 27. Choreco-atheloide Bewegungen der Hand & 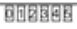 \\
\hline 28. Pilendehertremor & 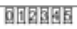 \\
\hline 29. Strecichen oder Reiben uber Gessicht und Hase & 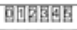 \\
\hline 30. Rebben der Coerschenket & 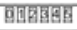 \\
\hline 31. Sonstbig: & 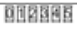 \\
\hline 32. Sonstbige & 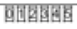 \\
\hline \multicolumn{2}{|l|}{ Umarero Extromitstan } \\
\hline 33. Rotabion undoder Flexion in den Sproungegereniken & 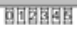 \\
\hline 34. Bewegungen der Zetien & 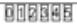 \\
\hline 35. Stamplende Bewegungen im Stethen & 可回目国目 \\
\hline 36. Stamplende Bewegungen im Sitzen & 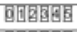 \\
\hline 37. Bewegungsumuhe der Bene (Restless legs") & 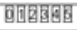 \\
\hline 38. Wedertothes Obereinanderschilagen der Beine im Sitzen & E回回目回圆 \\
\hline 39. Sorstope: & 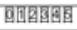 \\
\hline 40. Sonstipe & 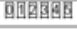 \\
\hline \multicolumn{2}{|l|}{ Körperhaltung } \\
\hline 41. Abrupte, zusarmentangende Benegungsoblusufe & 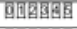 \\
\hline 42. Akathisise & 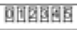 \\
\hline 43. Sonstoge: & 可四圂可目 \\
\hline 44. Sonstige: & 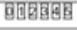 \\
\hline
\end{tabular}

seore:
Datum: Pat-cod
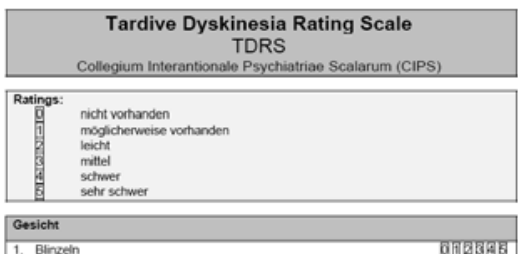

1. Blinzen

2. Lidzinem

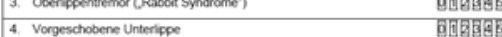

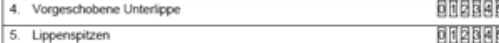

5. Lippenspitizen

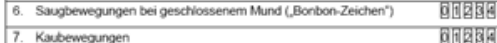

8. Schrasten

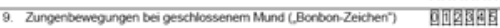

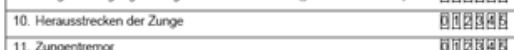

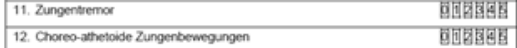

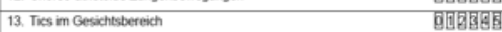

14. Grimassieren 可回国国国

15. Sorstipe:

16. Sonsoge:

Nacken und Rumpt

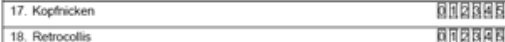

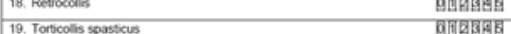

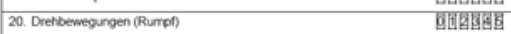

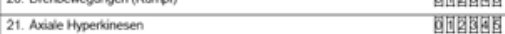
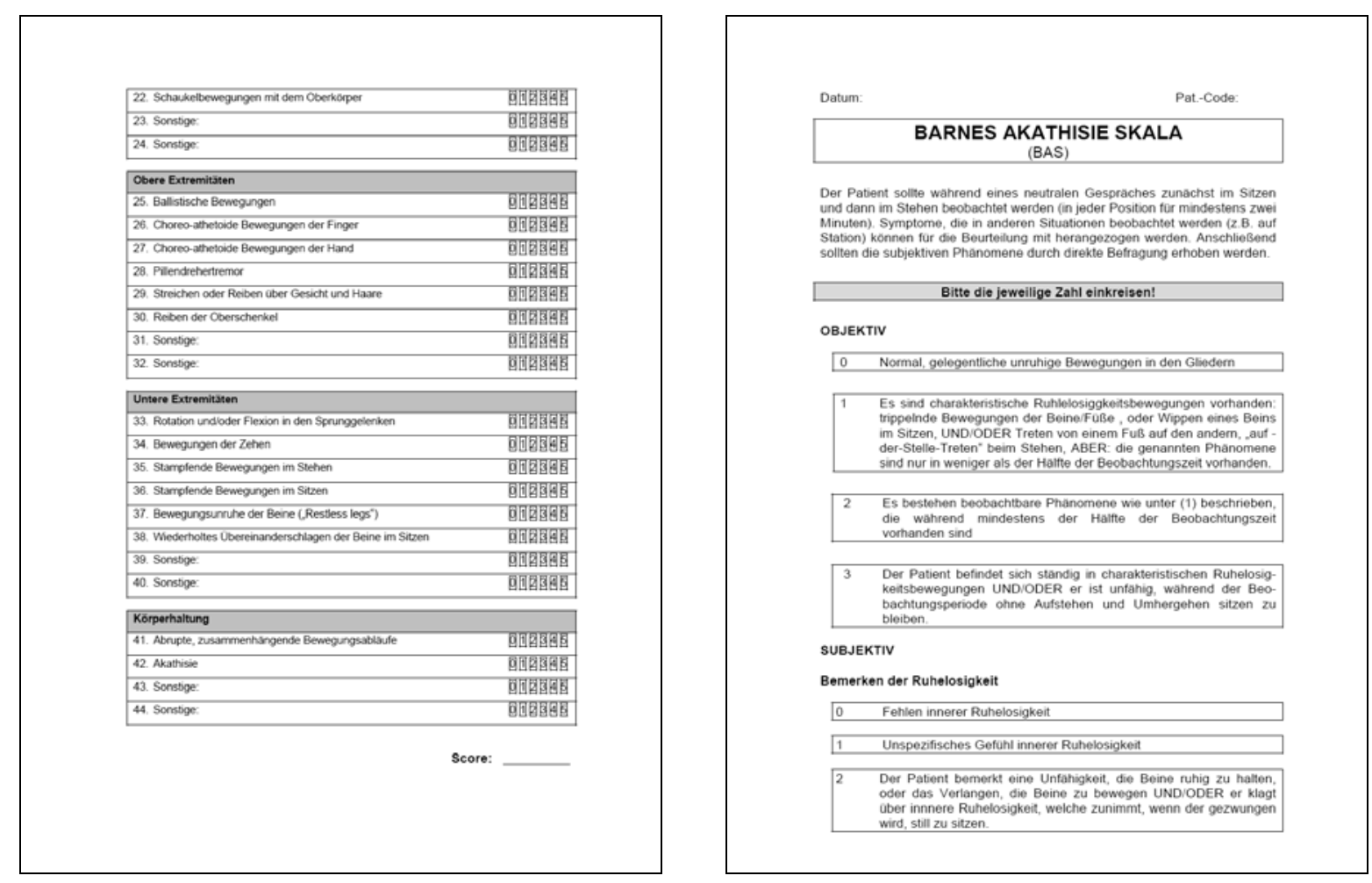

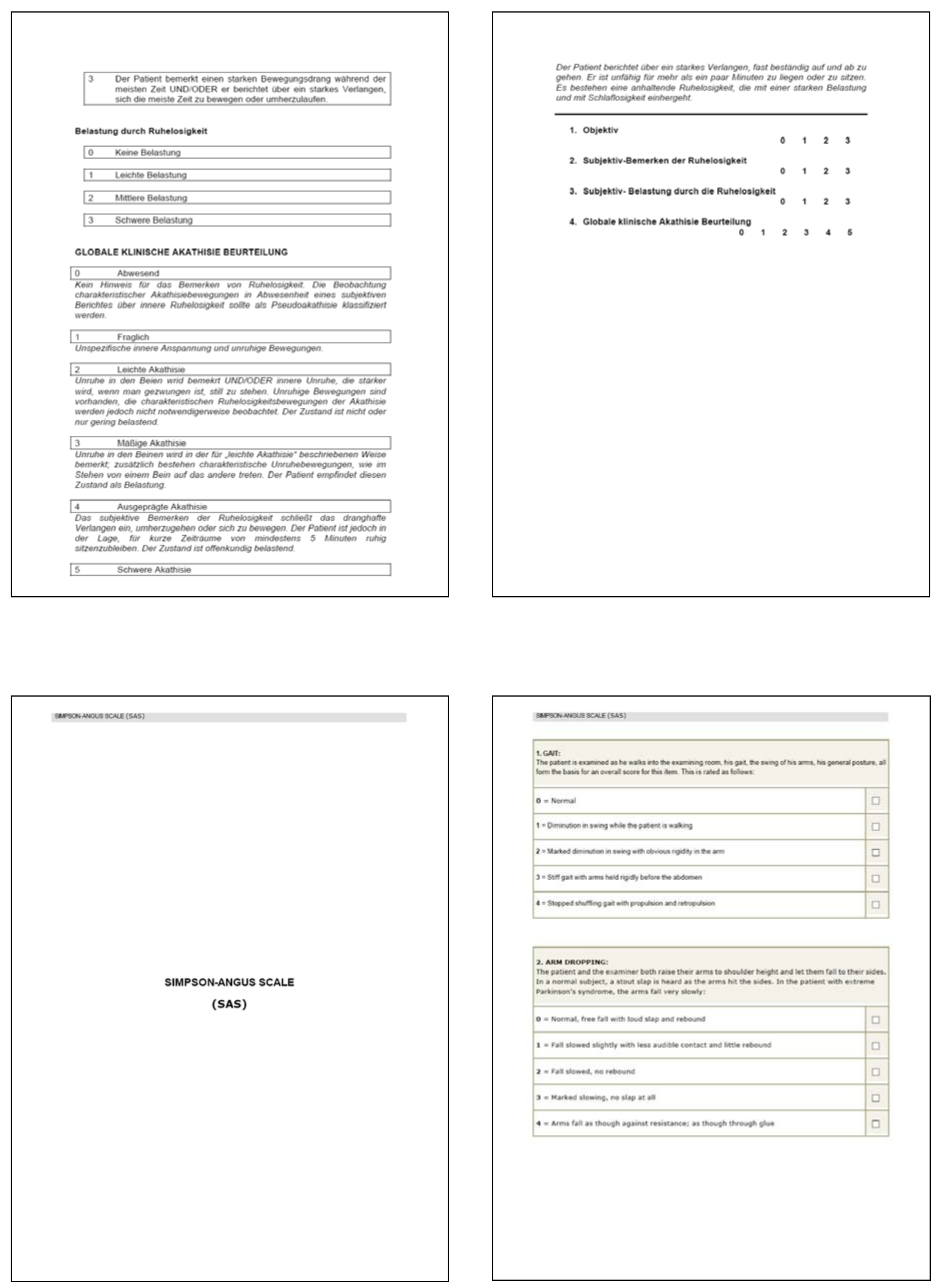

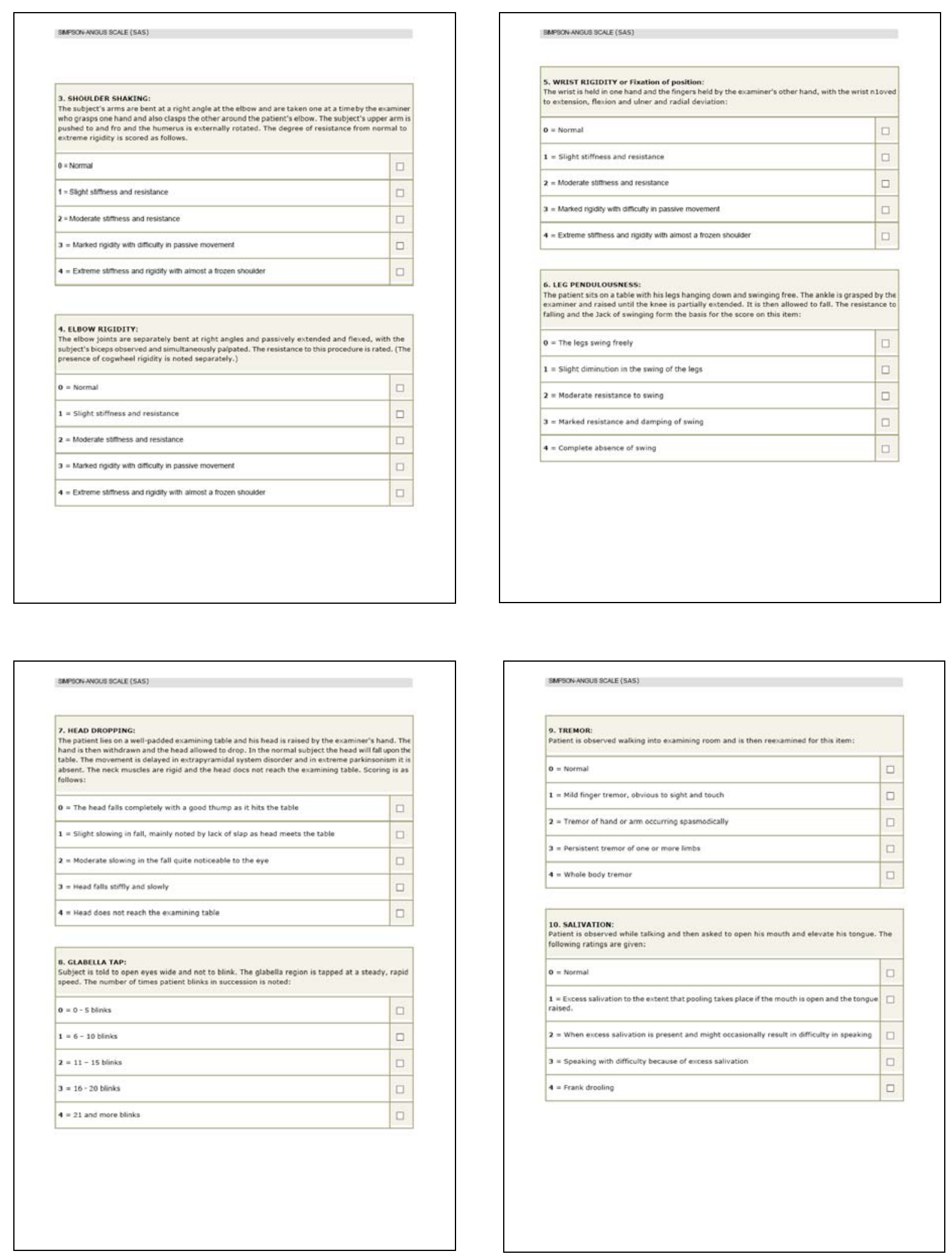
Daturn

Pat-code

\begin{tabular}{|c|c|c|}
\hline \multicolumn{3}{|c|}{$\begin{array}{c}\text { Tardive Dyskinesia Rating Scale } \\
\text { TDRS } \\
\text { Collegium Interantionale Psychiatriae Scalarum (CIPS) }\end{array}$} \\
\hline 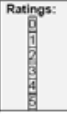 & $\begin{array}{l}\text { nicht vachanden } \\
\text { mogicherweise vorthansen } \\
\text { leesch } \\
\text { mintel } \\
\text { sctwer } \\
\text { sehr schiwer }\end{array}$ & \\
\hline \multicolumn{3}{|l|}{ Gessich } \\
\hline 1. Blinzel & & 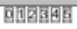 \\
\hline 2. Lidzence & & 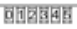 \\
\hline 3. Oberip & pentemor (Rabbil Syndrome") & 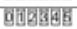 \\
\hline 4. Vorges & chobene Unterippe & 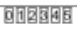 \\
\hline 5. Lippent & spizen & 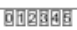 \\
\hline 6. Saugber & evegungen bei geschlosenenem Mund (Bcrbon-ZZeichen") & 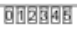 \\
\hline 7. Kauber & wegangen & 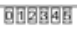 \\
\hline 8. Schras & zen & 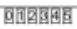 \\
\hline 9. Zunger & nbewegungen bei geschlosssenem Mund (BonbonnZZeichen) & 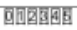 \\
\hline 10. Heraus & istrecken der Zunge & 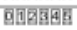 \\
\hline 11. Zunger & ntemox & 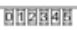 \\
\hline 12. Chared & -atheloiste Zungenterwegingen & 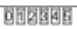 \\
\hline 13. Ties im & Qessichtsteréch & 可回逐四颔 \\
\hline 14. Gimas & essienten & 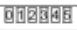 \\
\hline 15. Sonstip & & 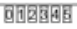 \\
\hline 16. Sonstig & & 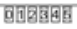 \\
\hline \multicolumn{3}{|c|}{ Nacken und Rumpt } \\
\hline 17. Koptric & & 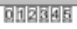 \\
\hline 18. Fetroce & & 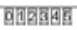 \\
\hline 19. Toricos & his spasscus & 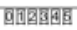 \\
\hline 20. Dreetber & Evegungen (Rumpa) & 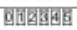 \\
\hline 21. Axise! & Hyperkinesen & 可自圂面喕 \\
\hline
\end{tabular}

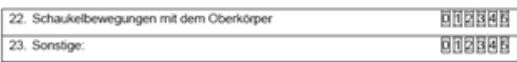

24. Sonstoge:

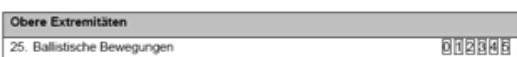

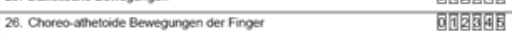

27. Chorea-sthetode Benegungen der Hand

28. Pilendrehertremar

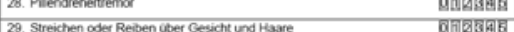

30 Reben of Oterschenkel

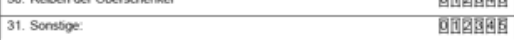

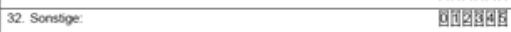

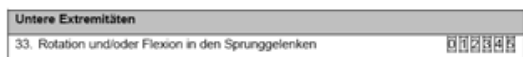

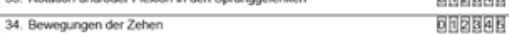

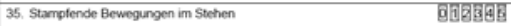

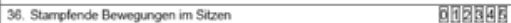

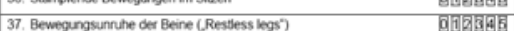

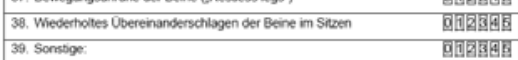

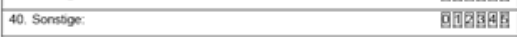

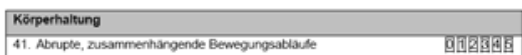

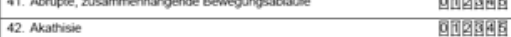

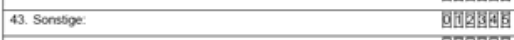

44. Sonsoge:

Score: 


\section{LITERATURVERZEICHNIS}

Addy N, Levin ED (2002): Nicotine interactions with haloperidol, clozapine and risperidone and working memory function in rats. Neuropsychopharmacology 27(4): 534-41

Aichhorn W, Gasser M, Weiss EM, Adlassning C, Marksteiner J (2005): Gender Differences in Pharmacokinetics and Side Effects of Second Generation Antipsychotic Drugs. Curr Neuropharmacol 3: 73-85

Aichhorn W, Marksteiner J, Walch T, Zernig G, Saria A, Kemmler G (2006): Influence of age, gender, body weight and valproate comedication on quetiapine plasma concentrations. Int Clin Psychopharmacol 21(2): 81-5

Aichhorn W, Whitworth AB, Weiss EM, Hinterhuber H, Marksteiner J (2007): [Differences between men and women in side effects of second-generation antipsychotics]. Nervenarzt 78(1): 45-52

Aktories K, Förstermann U, Hofmann F, Starke K: Allgemeine und spezielle Pharmakologie und Toxikologie, 10. Auflage, Urban \& Fischer, München 2009.

AmericanPsychiatricAssociation (1997): Practice guideline for the treatment of patients with schizophrenia. Am J Psychiatry 154(4 Suppl): 1-63

AmericanPsychiatricAssociation: Diagnostic and Statistical Manual of Mental Disorders: DSM-IV-TR, AmericanPsychiatricAssociation, Washington 2000, 312

Amin F, Stroe AE, Kahn T, Knott PJ, Kahn RS, Davidson M (1998): Control of renal factors in plasma homovanillic acid measurements. Neuropsychopharmacology 18(4): 317-20

Andia AM, Zisook S, Heaton RK, Hesselink J, Jernigan T, Kuck J, Morganville J, Braff DL (1995): Gender differences in schizophrenia. J Nerv Ment Dis 183(8): 522-8

Andreasen NC (1995): Symptoms, signs, and diagnosis of schizophrenia. Lancet 346(8973): 477-81

Andreasen NC, Olsen S (1982): Negative v positive schizophrenia. Definition and validation. Arch Gen Psychiatry 39(7): 789-94

Angermeyer MC, Kuhn L, Goldstein JM (1990): Gender and the course of schizophrenia: differences in treated outcomes. Schizophr Bull 16(2): 293-307

Antonova E, Sharma T, Morris R, Kumari V (2004): The relationship between brain structure and neurocognition in schizophrenia: a selective review. Schizophr Res 70(2-3): 11745

Artaloytia JF, Arango C, Lahti A, Sanz J, Pascual A, Cubero P, Prieto D, Palomo T (2006): Negative signs and symptoms secondary to antipsychotics: a double-blind, randomized trial of a single dose of placebo, haloperidol, and risperidone in healthy volunteers. Am J Psychiatry 163(3): 488-93

Aschenbrenner S, Tucha O, Lange K: Regensburger Wortflüssigkeitstest, Hogrefe, Göttingen 2000.

Ashcroft R: The Declaration of Helsinki; in: The Oxford Textbook of Clinical Research Ethics, 1. Edition; hrsg. v. Emanuel E, Grady C,Crouch R; Oxford University Press, New York 2007.

Barbui C, Nose M, Bindman J, Schene A, Becker T, Mazzi MA, Kikkert M, Camara J, Born A, Tansella M (2005): Sex differences in the subjective tolerability of antipsychotic drugs. J Clin Psychopharmacol 25(6): 521-6

Barker MJ, Greenwood KM, Jackson M, Crowe SF (2004): Persistence of cognitive effects after withdrawal from long-term benzodiazepine use: a meta-analysis. Arch Clin Neuropsychol 19(3): 437-54

Barnes TR (1989): A rating scale for drug-induced akathisia. Br J Psychiatry 154: 672-676

Bartels C, Mertens N, Hofer S, Merboldt KD, Dietrich J, Frahm J, Ehrenreich H (2008): Callosal dysfunction in amyotrophic lateral sclerosis correlates with diffusion tensor imaging of the central motor system. Neuromuscul Disord 18(5): 398-407 
Bartels SJ, Pratt SI (2009): Psychosocial rehabilitation and quality of life for older adults with serious mental illness: recent findings and future research directions. Curr Opin Psychiatry 22(4): $381-5$

Bayer TA, Falkai P, Maier W (1999): Genetic and non-genetic vulnerability factors in schizophrenia: the basis of the "two hit hypothesis". J Psychiatr Res 33(6): 543-8

Bleuler E: Dementia praecox oder Gruppe der Schizophrenien, F. Deuticke, Leipzig und Wien 1911.

Borsy J, Toldy L, Dumbovich B (1965): Neuroplegic and Other Pharmacological Properties of Methophenazine (Frenolon). Acta Physiol Acad Sci Hung 27: 65-80

Bortz J: Statistik für Sozialwissenschaftler, 5. Auflage, Springer-Verlag, Berlin 1999.

Bosse R, DiPaolo T (1996): The modulation of brain dopamine and GABAA receptors by estradiol: a clue for CNS changes occurring at menopause. Cell Mol Neurobiol 16(2): 199-212

Bowie CR, Reichenberg A, Patterson TL, Heaton RK, Harvey PD (2006): Determinants of real-world functional performance in schizophrenia subjects: correlations with cognition, functional capacity, and symptoms. Am J Psychiatry 163(3): 418-25

Bowie CR, Reichenberg A, McClure MM, Leung WL, Harvey PD (2008): Age-associated differences in cognitive performance in older community dwelling schizophrenia patients: differential sensitivity of clinical neuropsychological and experimental information processing tests. Schizophr Res 106(1): 50-8

Brandon NJ, Millar JK, Korth C, Sive H, Singh KK, Sawa A (2009): Understanding the role of DISC1 in psychiatric disease and during normal development. J Neurosci 29(41): 12768-75

Brann DW, Dhandapani K, Wakade C, Mahesh VB, Khan MM (2007): Neurotrophic and neuroprotective actions of estrogen: basic mechanisms and clinical implications. Steroids $\underline{72(5): 381-405}$

Bratti IM, Kane JM, Marder SR (2007): Chronic restlessness with antipsychotics. Am J Psychiatry 164(11): 1648-54

Breier A, Schreiber JL, Dyer J, Pickar D (1991): National Institute of Mental Health longitudinal study of chronic schizophrenia. Prognosis and predictors of outcome. Arch Gen Psychiatry 48(3): 239-46

Buchanan RW, Freedman R, Javitt DC, Abi-Dargham A, Lieberman JA (2007): Recent advances in the development of novel pharmacological agents for the treatment of cognitive impairments in schizophrenia. Schizophr Bull 33(5): 1120-30

Buckley PF, Stahl SM (2007): Pharmacological treatment of negative symptoms of schizophrenia: therapeutic opportunity or cul-de-sac? Acta Psychiatr Scand 115(2): 93-100

Bühl A: SPSS 14 Einführung in die moderne Datenanalyse, 10. Auflage, Pearson Studium, München 2006.

Burns T, Catty J, Dash M, Roberts C, Lockwood A, Marshall M (2007): Use of intensive case management to reduce time in hospital in people with severe mental illness: systematic review and meta-regression. BMJ 335(7615): 336

Cannon TD, Zorrilla LE, Shtasel D, Gur RE, Gur RC, Marco EJ, Moberg P, Price RA (1994): Neuropsychological functioning in siblings discordant for schizophrenia and healthy volunteers. Arch Gen Psychiatry 51(8): 651-61

Cardno AG, Gottesman, II (2000): Twin studies of schizophrenia: from bow-and-arrow concordances to star wars Mx and functional genomics. Am J Med Genet 97(1): 12-7

Casey DE (1991): Neuroleptic drug-induced extrapyramidal syndromes and tardive dyskinesia. Schizophr Res 4(2): 109-20

Castle DJ, Murray RM (1993): The epidemiology of late-onset schizophrenia. Schizophr Bull 19(4): 691-700

Centorrino F, Goren JL, Hennen J, Salvatore P, Kelleher JP, Baldessarini RJ (2004): Multiple versus single antipsychotic agents for hospitalized psychiatric patients: case-control study of risks versus benefits. Am J Psychiatry 161(4): 700-6 
Chakos MH, Schobel SA, Gu H, Gerig G, Bradford D, Charles C, Lieberman JA (2005): Duration of illness and treatment effects on hippocampal volume in male patients with schizophrenia. Br J Psychiatry 186: 26-31

Chakos MH, Glick ID, Miller AL, Hamner MB, Miller DD, Patel JK, Tapp A, Keefe RS, Rosenheck RA (2006): Baseline use of concomitant psychotropic medications to treat schizophrenia in the CATIE trial. Psychiatr Serv 57(8): 1094-101

Chapman RL (1948): The MacQuarrie test for mechanical ability. Psychometrika 13(3): 175179

Chaves AC, Seeman MV (2006): Sex selection bias in schizophrenia antipsychotic trials. J Clin Psychopharmacol 26(5): 489-94

Chen EY, Shapleske J, Luque R, MCKenna PJ, Hodges JR, Colloway SP (1995): The Cambridge Neurological Inventory: a clinical instrument for assessment of soft neurological signs in psychiatric patients. Psychiatry Res 56(2): 183-204

Citrome L, Jaffe A, Levine J (2005): Dosing of second-generation antipsychotic medication in a state hospital system. J Clin Psychopharmacol 25(4): 388-91

Citrome L, Stauffer VL, Chen L, Kinon BJ, Kurtz DL, Jacobson JG, Bergstrom RF (2009): Olanzapine plasma concentrations after treatment with 10,20 , and $40 \mathrm{mg} / \mathrm{d}$ in $\mathrm{pa}-$ tients with schizophrenia: an analysis of correlations with efficacy, weight gain, and prolactin concentration. J Clin Psychopharmacol 29(3): 278-83

Conley RR, Kelly DL (2005): Second-generation antipsychotics for schizophrenia: a review of clinical pharmacology and medication-associated side effects. Isr J Psychiatry Relat Sci 42(1): 51-60

Correll CU, Rummel-Kluge C, Corves C, Kane JM, Leucht S (2009): Antipsychotic combinations vs monotherapy in schizophrenia: a meta-analysis of randomized controlled trials. Schizophr Bull 35(2): 443-57

Crow TJ (1980): Positive and negative schizophrenic symptoms and the role of dopamine. $\mathrm{Br}$ J Psychiatry 137: 383-6

Dalack GW, Healy DJ, Meador-Woodruff JH (1998): Nicotine dependence in schizophrenia: clinical phenomena and laboratory findings. Am J Psychiatry 155(11): 1490-501

Davidson M, Galderisi S, Weiser M, Werbeloff N, Fleischhacker WW, Keefe RS, Boter H, Keet IP, Prelipceanu D, Rybakowski JK, et al. (2009): Cognitive effects of antipsychotic drugs in first-episode schizophrenia and schizophreniform disorder: a randomized, open-label clinical trial (EUFEST). Am J Psychiatry 166(6): 675-82

Davis JM, Chen N (2004): Dose response and dose equivalence of antipsychotics. J Clin Psychopharmacol 24(2): 192-208

Davis KL, Stewart DG, Friedman JI, Buchsbaum M, Harvey PD, Hof PR, Buxbaum J, Haroutunian V (2003): White matter changes in schizophrenia: evidence for myelinrelated dysfunction. Arch Gen Psychiatry 60(5): 443-56

de Leon J, Diaz FJ (2005): A meta-analysis of worldwide studies demonstrates an association between schizophrenia and tobacco smoking behaviors. Schizophr Res 76(2-3): 13557

DeLisi LE (2008): The concept of progressive brain change in schizophrenia: implications for understanding schizophrenia. Schizophr Bull 34(2): 312-21

Diaz E, Neuse E, Sullivan MC, Pearsall HR, Woods SW (2004): Adherence to conventional and atypical antipsychotics after hospital discharge. J Clin Psychiatry 65(3): 354-60

Didriksen M (1995): Effects of antipsychotics on cognitive behaviour in rats using the delayed non-match to position paradigm. Eur J Pharmacol 281(3): 241-50

Dilling H, Freyberger HJH: Taschenführer zur ICD-10-Klassifikation psychischer Störungen, 3. Edition, Huber, Bern 2006.

Dominguez Mde G, Viechtbauer W, Simons CJ, van Os J, Krabbendam L (2009): Are psychotic psychopathology and neurocognition orthogonal? A systematic review of their associations. Psychol Bull 135(1): 157-71 
Dorph-Petersen KA, Pierri JN, Perel JM, Sun Z, Sampson AR, Lewis DA (2005): The influence of chronic exposure to antipsychotic medications on brain size before and after tissue fixation: a comparison of haloperidol and olanzapine in macaque monkeys. Neuropsychopharmacology 30(9): 1649-61

D'Souza DC, Abi-Saab WM, Madonick S, Forselius-Bielen K, Doersch A, Braley G, Gueorguieva R, Cooper TB, Krystal JH (2005): Delta-9-tetrahydrocannabinol effects in schizophrenia: implications for cognition, psychosis, and addiction. Biol Psychiatry 57(6): 594-608

Eack SM, Hogarty GE, Cho RY, Prasad KM, Greenwald DP, Hogarty SS, Keshavan MS (2010): Neuroprotective effects of cognitive enhancement therapy against gray matter loss in early schizophrenia: results from a 2 -year randomized controlled trial. Arch Gen Psychiatry 67(7): 674-82

Eerdekens M, Van Hove I, Remmerie B, Mannaert E (2004): Pharmacokinetics and tolerability of long-acting risperidone in schizophrenia. Schizophr Res 70(1): 91-100

Ehrenreich H, Göttingen: Antipsychotische Dosis von Promethazin. Mündliche Mitteilung 2010

Elie D, Poirier M, Chianetta J, Durand M, Gregoire C, Grignon S (2010): Cognitive effects of antipsychotic dosage and polypharmacy: a study with the BACS in patients with schizophrenia and schizoaffective disorder. J Psychopharmacol 24(7): 1037-44

Elsabagh S, Premkumar P, Anilkumar AP, Kumari V (2009): A longer duration of schizophrenic illness has sex-specific associations within the working memory neural network in schizophrenia. Behav Brain Res 201(1): 41-7

Endicott J, Spitzer RL, Fleiss JL, Cohen J (1976): The global assessment scale. A procedure for measuring overall severity of psychiatric disturbance. Arch Gen Psychiatry 33(6): 766-71

Erlenmeyer-Kimling L, Rock D, Roberts SA, Janal M, Kestenbaum C, Cornblatt B, Adamo UH, Gottesman, II (2000): Attention, memory, and motor skills as childhood predictors of schizophrenia-related psychoses: the New York High-Risk Project. Am J Psychiatry 157(9): 1416-22

Faraone SV, Seidman LJ, Kremen WS, Toomey R, Lyons MJ, Tsuang MT (1996): Neuropsychological functioning among the elderly nonpsychotic relatives of schizophrenic patients. Schizophr Res 21(1): 27-31

Field AP: Discovering statistics Using SPSS: (and sex and drugs and rock'n'roll), 3. Edition, Sage Publications, Los Angeles 2009.

Fioravanti M, Carlone O, Vitale B, Cinti ME, Clare L (2005): A meta-analysis of cognitive deficits in adults with a diagnosis of schizophrenia. Neuropsychol Rev 15(2): 73-95

Flor-Henry P (1990): Influence of gender in schizophrenia as related to other psychopathological syndromes. Schizophr Bull 16(2): 211-27

Franke GH: Brief Symptom Inventory (BSI), Beltz Test, Göttingen 2000.

Fuller R, Nopoulos P, Arndt S, O'Leary D, Ho BC, Andreasen NC (2002): Longitudinal assessment of premorbid cognitive functioning in patients with schizophrenia through examination of standardized scholastic test performance. Am J Psychiatry 159(7): 1183-9

Gaebel W, Falkai P: Behandlungsleitlinie Schizophrenie, Steinkopff, Darmstadt 2005.

Geddes J, Freemantle N, Harrison P, Bebbington P (2000): Atypical antipsychotics in the treatment of schizophrenia: systematic overview and meta-regression analysis. BMJ 321(7273): 1371-6

Gilmer TP, Dolder CR, Folsom DP, Mastin W, Jeste DV (2007): Antipsychotic polypharmacy trends among Medicaid beneficiaries with schizophrenia in San Diego County, 19992004. Psychiatr Serv 58(7): 1007-10

Goff DC, Henderson DC, Amico E (1992): Cigarette smoking in schizophrenia: relationship to psychopathology and medication side effects. Am J Psychiatry 149(9): 1189-94 
Goldberg TE, Keefe RS, Goldman RS, Robinson DG, Harvey PD (2010): Circumstances under which practice does not make perfect: a review of the practice effect literature in schizophrenia and its relevance to clinical treatment studies. Neuropsychopharmacology 35(5): 1053-62

Goldstein JM, Seidman LJ, Goodman JM, Koren D, Lee H, Weintraub S, Tsuang MT (1998): Are there sex differences in neuropsychological functions among patients with schizophrenia? Am J Psychiatry 155(10): 1358-64

Gottesman I: Schizophrenia Genesis The Origins of Madness, W. H. Freeman and Company, New York 1991.

Green MF (1996 a): What are the functional consequences of neurocognitive deficits in schizophrenia? Am J Psychiatry 153(3): 321-30

Green MF (1996 b): Cognitive impairment and functional outcome in schizophrenia and bipolar disorder. J Clin Psychiatry 67 Suppl 9: 3-8; discussion 36-42

Green MF, Nuechterlein KH (2004): The MATRICS initiative: developing a consensus cognitive battery for clinical trials. Schizophr Res 72(1): 1-3

Green MF, Kern RS, Heaton RK (2004): Longitudinal studies of cognition and functional outcome in schizophrenia: implications for MATRICS. Schizophr Res 72(1): 41-51

Gur RE, Gur RC (1990): Gender differences in regional cerebral blood flow. Schizophr Bull 16(2): 247-54

Guy W: ECDEU Assessment Manual for Psychopharmacology, Revised, MD, Rockville 1976.

Haase H-J: Neuroleptika - neuroleptische Potenz; in: Therapie mit Psychopharmaka und anderen psychotropen Medikamenten; hrsg. v. Haase H-J; Gerhard Stalling AG, Oldenburg 1969, 98 - 132.

Haase H-J, Janssen PA: The Action of Neuroleptic Drugs, North-Holland Publishing Company, Amsterdam 1965.

Hafner H (2003): Gender differences in schizophrenia. Psychoneuroendocrinology 28 Suppl 2: $17-54$

Hafner H, an der Heiden W, Behrens S, Gattaz WF, Hambrecht M, Loffler W, Maurer K, Munk-Jorgensen P, Nowotny B, Riecher-Rossler A, et al. (1998): Causes and consequences of the gender difference in age at onset of schizophrenia. Schizophr Bull 24(1): 99-113

Häfner H. Das Rätsel Schizophrenie, C.H. Beck, München 2004.

Harrison PJ, Law AJ (2006): Neuregulin 1 and schizophrenia: genetics, gene expression, and neurobiology. Biol Psychiatry 60(2): 132-40

Harrison PJ, Freemantle N, Geddes JR (2003): Meta-analysis of brain weight in schizophrenia. Schizophr Res 64(1): 25-34

Harrow M, Jobe TH (2007): Factors involved in outcome and recovery in schizophrenia patients not on antipsychotic medications: a 15-year multifollow-up study. J Nerv Ment Dis 195(5): 406-14

Harvey PD, Keefe RS (2001): Studies of cognitive change in patients with schizophrenia following novel antipsychotic treatment. Am J Psychiatry 158(2): 176-84

Harvey PD, Lombardi J, Leibman M, White L, Parrella M, Powchik P, Davidson M (1996): Cognitive impairment and negative symptoms in geriatric chronic schizophrenic patients: a follow-up study. Schizophr Res 22(3): 223-31

Harvey PD, Green MF, Keefe RS, Velligan DI (2004): Cognitive functioning in schizophrenia: a consensus statement on its role in the definition and evaluation of effective treatments for the illness. J Clin Psychiatry 65(3): 361-72

Harvey PD, Koren D, Reichenberg A, Bowie CR (2006): Negative symptoms and cognitive deficits: what is the nature of their relationship? Schizophr Bull 32(2): 250-8

Harvey PD, Reichenberg A, Bowie CR, Patterson TL, Heaton RK (2010): The course of neuropsychological performance and functional capacity in older patients with schizophrenia: influences of previous history of long-term institutional stay. Biol Psychiatry 67(10): 933-9 
Heinrichs RW, Zakzanis KK (1998): Neurocognitive deficit in schizophrenia: a quantitative review of the evidence. Neuropsychology 12(3): 426-45

Helmstädter C, Lendt M, Lux S: Verbaler Lern- und Merkfähigkeitstest, Beltz Test, Göttingen 2001.

Hertz L (2004): Intercellular metabolic compartmentation in the brain: past, present and future. Neurochem Int 45(2-3): 285-96

Hinrichs JV, Mewaldt SP, Ghoneim MM, Berie JL (1982): Diazepam and learning: assessment of acquisition deficits. Pharmacol Biochem Behav 17(1): 165-70

Ho BC, Andreasen NC, Ziebell S, Pierson R, Magnotta V (2011): Long-term Antipsychotic Treatment and Brain Volumes: A Longitudinal Study of First-Episode Schizophrenia. Arch Gen Psychiatry 68(2): 128-37

Hoff AL, Faustman WO, Wieneke M, Espinoza S, Costa M, Wolkowitz O, Csernansky JG (1996): The effects of clozapine on symptom reduction, neurocognitive function, and clinical management in treatment-refractory state hospital schizophrenic inpatients. Neuropsychopharmacology 15(4): 361-9

Hoff AL, Svetina C, Shields G, Stewart J, DeLisi LE (2005): Ten year longitudinal study of neuropsychological functioning subsequent to a first episode of schizophrenia. Schizophr Res 78(1): 27-34

Hoffman RE, McGlashan TH (1993): Neurodynamics and schizophrenia research: editors' introduction. Schizophr Bull 19(1): 15-9

Honer WG, Thornton AE, Chen EY, Chan RC, Wong JO, Bergmann A, Falkai P, PomarolClotet E, McKenna PJ, Stip E, et al. (2006): Clozapine alone versus clozapine and risperidone with refractory schizophrenia. N Engl J Med 354(5): 472-82

Horan WP, Blanchard JJ (2003): Neurocognitive, social, and emotional dysfunction in deficit syndrome schizophrenia. Schizophr Res 65(2-3): 125-37

Hori H, Noguchi H, Hashimoto R, Nakabayashi T, Omori M, Takahashi S, Tsukue R, Anami K, Hirabayashi N, Harada S, et al. (2006): Antipsychotic medication and cognitive function in schizophrenia. Schizophr Res 86(1-3): 138-46

Horn W: Leistungsprüfsystem (LPS), 2. Auflage, Hogrefe, Göttingen 1983.

Hughes JR, Hatsukami DK, Mitchell JE, Dahlgren LA (1986): Prevalence of smoking among psychiatric outpatients. Am J Psychiatry 143(8): 993-7

Hulshoff Pol HE, Kahn RS (2008): What happens after the first episode? A review of progressive brain changes in chronically ill patients with schizophrenia. Schizophr Bull 34(2): 354-66

Hyman SE, Arana GW, Rosenbaum JF: Handbook of Psychiatric Drug Therapie, 3. Edition, Lippincott Williams \& Wilkins, Washington 1995.

Ito H, Koyama A, Higuchi T (2005): Polypharmacy and excessive dosing: psychiatrists' perceptions of antipsychotic drug prescription. Br J Psychiatry 187: 243-7

Jablensky A, Sartorius N, Ernberg G, Anker M, Korten A, Cooper JE, Day R, Bertelsen A (1992): Schizophrenia: manifestations, incidence and course in different cultures. A World Health Organization ten-country study. Psychol Med Monogr Suppl 20: 1-97

Jobe TH, Harrow M (2005): Long-term outcome of patients with schizophrenia: a review. Can J Psychiatry 50(14): 892-900

Johnstone EC, Owens DG, Gold A, Crow TJ, MacMillan JF (1981): Institutionalization and the defects of schizophrenia. Br J Psychiatry 139: 195-203

Jones PB, Rantakallio P, Hartikainen AL, Isohanni M, Sipila P (1998): Schizophrenia as a long-term outcome of pregnancy, delivery, and perinatal complications: a 28 -year follow-up of the 1966 north Finland general population birth cohort. Am J Psychiatry 155(3): 355-64

Joukamaa M, Heliovaara M, Knekt P, Aromaa A, Raitasalo R, Lehtinen V (2006): Schizophrenia, neuroleptic medication and mortality. Br J Psychiatry 188: 122-7

Kane JM, Sharif ZA (2008): Atypical antipsychotics: sedation versus efficacy. J Clin Psychiatry 69 Suppl 1: 18-31 
Kane JM, Aguglia E, Altamura AC, Ayuso Gutierrez JL, Brunello N, Fleischhacker WW, Gaebel W, Gerlach J, Guelfi JD, Kissling W, et al. (1998): Guidelines for depot antipsychotic treatment in schizophrenia. European Neuropsychopharmacology Consensus Conference in Siena, Italy. Eur Neuropsychopharmacol 8(1): 55-66

Kane JM, Leucht S, Carpenter D, Docherty JP (2003): The expert consensus guideline series. Optimizing pharmacologic treatment of psychotic disorders. Introduction: methods, commentary, and summary. J Clin Psychiatry 64 Suppl 12: 5-19

Kane JM, Perlis RH, Malhotra AK (2008): Clinical insights into pharmacogenetics and schizophrenia, part 2. J Clin Psychiatry 69(6): 1006-13

Kaplan HI, Sadock BJ. Pocket Handbook of Psychiatric Drug Treatment, 1st, Lippincott Williams \& Wilkins, Washington 1993.

Kapur S, Mamo D (2003): Half a century of antipsychotics and still a central role for dopamine D2 receptors. Prog Neuropsychopharmacol Biol Psychiatry 27(7): 1081-90

Kawai N, Yamakawa Y, Baba A, Nemoto K, Tachikawa H, Hori T, Asada T, Iidaka T (2006): High-dose of multiple antipsychotics and cognitive function in schizophrenia: the effect of dose-reduction. Prog Neuropsychopharmacol Biol Psychiatry 30(6): 1009-14

Kay SR, Fiszbein A, Opler LA (1987): The positive and negative syndrome scale (PANSS) for schizophrenia. Schizophr Bull 13(2): 261-276

Keefe RS, Fenton WS (2007): How should DSM-V criteria for schizophrenia include cognitive impairment? Schizophr Bull 33(4): 912-20

Keefe RS, Goldberg TE, Harvey PD, Gold JM, Poe MP, Coughenour L (2004): The Brief Assessment of Cognition in Schizophrenia: reliability, sensitivity, and comparison with a standard neurocognitive battery. Schizophr Res 68(2-3): 283-97

Keefe RS, Bilder RM, Davis SM, Harvey PD, Palmer BW, Gold JM, Meltzer HY, Green MF, Capuano G, Stroup TS, et al. (2007): Neurocognitive effects of antipsychotic medications in patients with chronic schizophrenia in the CATIE Trial. Arch Gen Psychiatry 64(6): 633-47

Kern RS, Nuechterlein KH, Green MF, Baade LE, Fenton WS, Gold JM, Keefe RS, Mesholam-Gately R, Mintz J, Seidman LJ, et al. (2008): The MATRICS Consensus Cognitive Battery, part 2: co-norming and standardization. Am J Psychiatry 165(2): 214-20

Kirkpatrick B, Alphs L, Buchanan RW (1992): The concept of supersensitivity psychosis. J Nerv Ment Dis 180(4): 265-70

Kirkpatrick B, Messias E, Harvey PD, Fernandez-Egea E, Bowie CR (2008): Is schizophrenia a syndrome of accelerated aging? Schizophr Bull 34(6): 1024-32

Kleinknecht RA, Donaldson D (1975): A review of the effects of diazepam on cognitive and psychomotor performance. J Nerv Ment Dis 161(6): 399-414

Konopaske GT, Dorph-Petersen KA, Sweet RA, Pierri JN, Zhang W, Sampson AR, Lewis DA (2008): Effect of chronic antipsychotic exposure on astrocyte and oligodendrocyte numbers in macaque monkeys. Biol Psychiatry 63(8): 759-65

Krabbendam L, van Os J (2005): Schizophrenia and urbanicity: a major environmental influence--conditional on genetic risk. Schizophr Bull 31(4): 795-9

Kraepelin E. Dementia Praecox and Paraphrenia, E. \& S. Livingstone, Edinburgh 1919.

Kubicki M, Niznikiewicz M, Connor E, Nestor P, Bouix S, Dreusicke M, Kikinis R, McCarley R, Shenton M (2009): Relationship Between White Matter Integrity, Attention, and Memory in Schizophrenia: A Diffusion Tensor Imaging Study. Brain Imaging Behav 3(2): 191-201

Kulkarni J, de Castella A, Smith D, Taffe J, Keks N, Copolov D (1996): A clinical trial of the effects of estrogen in acutely psychotic women. Schizophr Res 20(3): 247-52

Kulkarni J, de Castella A, Fitzgerald PB, Gurvich CT, Bailey M, Bartholomeusz C, Burger H (2008): Estrogen in severe mental illness: a potential new treatment approach. Arch Gen Psychiatry 65(8): 955-60 
Kupfer J, Brosis B, Braehler E. Toront-Alexithymia-Scale-26 (TAS-26), Hogrefe, Göttingen 2001.

Kurtz MM (2005): Neurocognitive impairment across the lifespan in schizophrenia: an update. Schizophr Res 74(1): 15-26

Larsen TK, McGlashan TH, Johannessen JO, Vibe-Hansen L (1996): First-episode schizophrenia: II. Premorbid patterns by gender. Schizophr Bull 22(2): 257-69

Laux L, Glanzmann P, Schaffner P, Spielberger CD. State-Trait-Anxiety-Inventory (STAI), Beltz Test, Weinheim 1981.

Lehman AF, Kreyenbuhl J, Buchanan RW, Dickerson FB, Dixon LB, Goldberg R, GreenPaden LD, Tenhula WN, Boerescu D, Tek C, et al. (2004): The Schizophrenia Patient Outcomes Research Team (PORT): updated treatment recommendations 2003. Schizophr Bull 30(2): 193-217

Lehrl S: Mehrfachwahl-Wortschatz-Intelligenztest MWT-B, Spitta Verlag, Balingen 1999.

Leon J, Susce MT, Pan RM, Wedlund PJ, Orrego ML, Diaz FJ (2007): A study of genetic (CYP2D6 and ABCB1) and environmental (drug inhibitors and inducers) variables that may influence plasma risperidone levels. Pharmacopsychiatry 40(3): 93-102

Leucht S, Pitschel-Walz G, Abraham D, Kissling W (1999): Efficacy and extrapyramidal sideeffects of the new antipsychotics olanzapine, quetiapine, risperidone, and sertindole compared to conventional antipsychotics and placebo. A meta-analysis of randomized controlled trials. Schizophr Res 35(1): 51-68

Leucht S, Arbter D, Engel RR, Kissling W, Davis JM (2009 a): How effective are secondgeneration antipsychotic drugs? A meta-analysis of placebo-controlled trials. Mol Psychiatry 14(4): 429-47

Leucht S, Corves C, Arbter D, Engel RR, Li C, Davis JM (2009 b): Second-generation versus first-generation antipsychotic drugs for schizophrenia: a meta-analysis. Lancet 373(9657): 31-41

Leung A, Chue P (2000): Sex differences in schizophrenia, a review of the literature. Acta Psychiatr Scand Suppl 401: 3-38

Levin ED, Wilson W, Rose JE, McEvoy J (1996): Nicotine-haloperidol interactions and cognitive performance in schizophrenics. Neuropsychopharmacology 15(5): 429-36

Lewis DA, Hashimoto T, Volk DW (2005): Cortical inhibitory neurons and schizophrenia. Nat Rev Neurosci 6(4): 312-24

Liddle PF (1987): Schizophrenic syndromes, cognitive performance and neurological dysfunction. Psychol Med 17(1): 49-57

Lieberman JA, Perkins D, Belger A, Chakos M, Jarskog F, Boteva K, Gilmore J (2001): The early stages of schizophrenia: speculations on pathogenesis, pathophysiology, and therapeutic approaches. Biol Psychiatry 50(11): 884-97

Lieberman JA, Stroup TS, McEvoy JP, Swartz MS, Rosenheck RA, Perkins DO, Keefe RS, Davis SM, Davis CE, Lebowitz BD, et al. (2005): Effectiveness of antipsychotic drugs in patients with chronic schizophrenia. N Engl J Med 353(12): 1209-23

Lienert GA, Raatz U: Testaufbau und Testanalyse, 6. Auflage, Beltz Psychologie Verlags Union, Weinheim 1998.

Lipkovich I, Adams DH, Mallinckrodt C, Faries D, Baron D, Houston JP (2008): Evaluating dose response from flexible dose clinical trials. BMC Psychiatry $\underline{8}: 3$

Longenecker J, Dickinson D, Weinberger DR, Elvevag B (2010): Cognitive differences between men and women: a comparison of patients with schizophrenia and healthy volunteers. Schizophr Res 120(1-3): 234-5

MacQuarrie TW: MacQuarrie Test for Mechanical Ability, CTB/McGraw-Hill, Monterey 1925, 1953.

McEvoy JP, Schooler NR, Wilson WH (1991): Predictors of therapeutic response to haloperidol in acute schizophrenia. Psychopharmacol Bull 27(2): 97-101

McGlashan TH, Hoffman RE (2000): Schizophrenia as a disorder of developmentally reduced synaptic connectivity. Arch Gen Psychiatry 57(7): 637-48 
McGlone J (1978): Sex differences in functional brain asymmetry. Cortex 14(1): 122-8

McGrath J, Saha S, Welham J, El Saadi O, MacCauley C, Chant D (2004): A systematic review of the incidence of schizophrenia: the distribution of rates and the influence of sex, urbanicity, migrant status and methodology. BMC Med 2: 13

McGrath J, Saha S, Chant D, Welham J (2008): Schizophrenia: a concise overview of incidence, prevalence, and mortality. Epidemiol Rev $\underline{\text { 30: 67-76 }}$

McGurk SR, Twamley EW, Sitzer DI, McHugo GJ, Mueser KT (2007): A meta-analysis of cognitive remediation in schizophrenia. Am J Psychiatry 164(12): 1791-802

McLaren S, Cookson JC, Silverstone T (1992): Positive and negative symptoms, depression and social disability in chronic schizophrenia: a comparative trial of bromperidol and fluphenazine decanoates. Int Clin Psychopharmacol 7(2): 67-72

Menezes NM, Arenovich T, Zipursky RB (2006): A systematic review of longitudinal outcome studies of first-episode psychosis. Psychol Med 36(10): 1349-62

Milev P, Ho BC, Arndt S, Andreasen NC (2005): Predictive values of neurocognition and negative symptoms on functional outcome in schizophrenia: a longitudinal firstepisode study with 7-year follow-up. Am J Psychiatry 162(3): 495-506

Miller AL, Hall CS, Buchanan RW, Buckley PF, Chiles JA, Conley RR, Crismon ML, Ereshefsky L, Essock SM, Finnerty M, et al. (2004): The Texas Medication Algorithm Project antipsychotic algorithm for schizophrenia: 2003 update. J Clin Psychiatry 65(4): 500-8

Minzenberg MJ, Poole JH, Benton C, Vinogradov S (2004): Association of anticholinergic load with impairment of complex attention and memory in schizophrenia. Am J Psychiatry 161(1): 116-24

Mirnics K, Middleton FA, Lewis DA, Levitt P (2001): Analysis of complex brain disorders with gene expression microarrays: schizophrenia as a disease of the synapse. Trends Neurosci 24(8): 479-86

Mishara AL, Goldberg TE (2004): A meta-analysis and critical review of the effects of conventional neuroleptic treatment on cognition in schizophrenia: opening a closed book. Biol Psychiatry 55(10): 1013-22

Mojtabai R, Olfson M (2010): National trends in psychotropic medication polypharmacy in office-based psychiatry. Arch Gen Psychiatry 67(1): 26-36

Mouaffak F, Tranulis C, Gourevitch R, Poirier MF, Douki S, Olie JP, Loo H, Gourion D (2006): Augmentation strategies of clozapine with antipsychotics in the treatment of ultraresistant schizophrenia. Clin Neuropharmacol 29(1): 28-33

Müller P, Göttingen: Neuroleptische Äquivalenzdosen. Schriftliche Mitteilung 1987

Müller P: Grundzüge neuroleptischer Behandlung; in: Therapie der Schizophrenie. Integrative Behandlung in Klinik, Praxis und Rehabilitation; hrsg. v. Müller P; Georg Thieme Verlag, Stuttgart - New York 1999, 36-59.

Müller WE: Pharmakologische und neurobiologische Grundlagen; in: Neuroleptika Pharmakologische Grundlagen, klinisches Wissen und therapeutisches Vorgehen; hrsg. v. Möller H-J, Müller WE,Bandelow B; Wissenschaftliche Verlagsgesellschaft mbH, Stuttgart 2001, 1-63.

Muller MJ, Wetzel H (1998): Improvement of inter-rater reliability of PANSS items and subscales by a standardized rater training. Acta Psychiatr Scand 98(2): 135-9

Murphy BL, Roth RH, Arnsten AF (1997): Clozapine reverses the spatial working memory deficits induced by FG7142 in monkeys. Neuropsychopharmacology 16(6): 433-7

Nielsen AS, Mortensen PB, O'Callaghan E, Mors O, Ewald H (2002): Is head injury a risk factor for schizophrenia? Schizophr Res 55(1-2): 93-8

Norton N, Williams HJ, Owen MJ (2006): An update on the genetics of schizophrenia. Curr Opin Psychiatry 19(2): 158-64

Ogden CL, Fryar CD, Carroll MD, Flegal KM (2004): Mean body weight, height, and body mass index, United States 1960-2002. Adv Data(347): 1-17 
Ohara K, Tanabu S, Yoshida K, Ishibashi K, Ikemoto K, Shibuya H (2003): Effects of smoking and cytochrome P450 2D6*10 allele on the plasma haloperidol concentration/dose ratio. Prog Neuropsychopharmacol Biol Psychiatry 27(6): 945-9

Oie M, Sundet K, Rund BR (2010): Neurocognitive decline in early-onset schizophrenia compared with ADHD and normal controls: evidence from a 13-year follow-up study. Schizophr Bull 36(3): 557-65

Owen AM, McMillan KM, Laird AR, Bullmore E (2005): N-back working memory paradigm: a meta-analysis of normative functional neuroimaging studies. Hum Brain Mapp 25(1): 46-59

Owens DC, Johnstone EC, Miller P, Macmillan JF, Crow TJ (2010): Duration of untreated illness and outcome in schizophrenia: test of predictions in relation to relapse risk. $\mathrm{Br}$ J Psychiatry 196: 296-301

Patel JK, Buckley PF, Woolson S, Hamer RM, McEvoy JP, Perkins DO, Lieberman JA (2009): Metabolic profiles of second-generation antipsychotics in early psychosis: findings from the CAFE study. Schizophr Res 111(1-3): 9-16

Pearce BD (2001): Schizophrenia and viral infection during neurodevelopment: a focus on mechanisms. Mol Psychiatry 6(6): 634-46

Pearlson GD, Folley BS (2008): Schizophrenia, psychiatric genetics, and Darwinian psychiatry: an evolutionary framework. Schizophr Bull 34(4): 722-33

Perkins DO, Gu H, Boteva K, Lieberman JA (2005): Relationship between duration of untreated psychosis and outcome in first-episode schizophrenia: a critical review and meta-analysis. Am J Psychiatry 162(10): 1785-804

Perlstein WM, Dixit NK, Carter CS, Noll DC, Cohen JD (2003): Prefrontal cortex dysfunction mediates deficits in working memory and prepotent responding in schizophrenia. Biol Psychiatry 53(1): 25-38

Pillon B, Czernecki V, Dubois B (2003): Dopamine and cognitive function. Curr Opin Neurol 16 Suppl 2: S17-22

Pinals DA, Malhotra AK, Missar CD, Pickar D, Breier A (1996): Lack of gender differences in neuroleptic response in patients with schizophrenia. Schizophr Res 22(3): 215-22

Pohjalainen T, Rinne JO, Nagren K, Syvalahti E, Hietala J (1998): Sex differences in the striatal dopamine D2 receptor binding characteristics in vivo. Am J Psychiatry 155(6): 768-73

Pollock BG (1997): Gender differences in psychotropic drug metabolism. Psychopharmacol Bull 33(2): 235-41

Quitkin F, Rifkin A, Klein DF (1976): Neurologic soft signs in schizophrenia and character disorders. Organicity in schizophrenia with premorbid asociality and emotionally unstable character disorders. Arch Gen Psychiatry 33(7): 845-53

Rabiner CJ, Wegner JT, Kane JM (1986): Outcome study of first-episode psychosis. I: Relapse rates after 1 year. Am J Psychiatry 143(9): 1155-8

Rajji TK, Ismail Z, Mulsant BH (2009): Age at onset and cognition in schizophrenia: metaanalysis. Br J Psychiatry 195(4): 286-93

Rapoport JL, Giedd J, Kumra S, Jacobsen L, Smith A, Lee P, Nelson J, Hamburger S (1997): Childhood-onset schizophrenia. Progressive ventricular change during adolescence. Arch Gen Psychiatry 54(10): 897-903

Reichenberg A, Weiser M, Caspi A, Knobler HY, Lubin G, Harvey PD, Rabinowitz J, Davidson M (2006): Premorbid intellectual functioning and risk of schizophrenia and spectrum disorders. J Clin Exp Neuropsychol 28(2): 193-207

Reichenberg A, Caspi A, Harrington H, Houts R, Keefe RS, Murray RM, Poulton R, Moffitt TE (2010): Static and dynamic cognitive deficits in childhood preceding adult schizophrenia: a 30-year study. Am J Psychiatry 167(2): 160-9

Reitan RM (1958): The validity of the Trail Making Test as an indicator of organic brain damage. Percept Motor Skills $\underline{8}: 271-276$ 
Rey MJ, Schulz P, Costa C, Dick P, Tissot R (1989): Guidelines for the dosage of neuroleptics. I: Chlorpromazine equivalents of orally administered neuroleptics. Int Clin Psychopharmacol 4(2): 95-104

Rezvani AH, Levin ED (2001): Cognitive effects of nicotine. Biol Psychiatry 49(3): 258-67

Riecher-Rossler A, Hafner H, Dutsch-Strobel A, Oster M, Stumbaum M, van Gulick-Bailer M, Loffler W (1994): Further evidence for a specific role of estradiol in schizophrenia? Biol Psychiatry $\underline{36(7): 492-4}$

Ring N, Tantam D, Montague L, Newby D, Black D, Morris J (1991): Gender differences in the incidence of definite schizophrenia and atypical psychosis--focus on negative symptoms of schizophrenia. Acta Psychiatr Scand 84(6): 489-96

Robinson D, Woerner MG, Alvir JM, Bilder R, Goldman R, Geisler S, Koreen A, Sheitman B, Chakos M, Mayerhoff D, et al. (1999 a): Predictors of relapse following response from a first episode of schizophrenia or schizoaffective disorder. Arch Gen Psychiatry 56(3): $241-7$

Robinson D, Woerner MG, Alvir JM, Geisler S, Koreen A, Sheitman B, Chakos M, Mayerhoff D, Bilder R, Goldman R, et al. (1999 b): Predictors of treatment response from a first episode of schizophrenia or schizoaffective disorder. Am J Psychiatry 156(4): 544-9

Rosenberg L, Park S (2002): Verbal and spatial functions across the menstrual cycle in healthy young women. Psychoneuroendocrinology 27(7): 835-41

Rossi A, De Cataldo S, Di Michele V, Manna V, Ceccoli S, Stratta P, Casacchia M (1990): Neurological soft signs in schizophrenia. Br J Psychiatry 157: 735-9

Rubin LH, Haas GL, Keshavan MS, Sweeney JA, Maki PM (2008): Sex difference in cognitive response to antipsychotic treatment in first episode schizophrenia. Neuropsychopharmacology 33(2): 290-7

Rund BR (1998): A review of longitudinal studies of cognitive functions in schizophrenia patients. Schizophr Bull 24(3): 425-35

Rusch N, Spoletini I, Wilke M, Bria P, Di Paola M, Di Iulio F, Martinotti G, Caltagirone C, Spalletta G (2007): Prefrontal-thalamic-cerebellar gray matter networks and executive functioning in schizophrenia. Schizophr Res 93(1-3): 79-89

Saha S, Chant D, McGrath J (2007): A systematic review of mortality in schizophrenia: is the differential mortality gap worsening over time? Arch Gen Psychiatry 64(10): 1123-31

Salokangas RK (2004): Gender and the use of neuroleptics in schizophrenia. Schizophr Res 66(1): 41-9

Salthouse TA (2010): Selective review of cognitive aging. J Int Neuropsychol Soc 16(5): 75460

Samaha AN, Seeman P, Stewart J, Rajabi H, Kapur S (2007): "Breakthrough" dopamine supersensitivity during ongoing antipsychotic treatment leads to treatment failure over time. J Neurosci 27(11): 2979-86

Sanchez MG, Bourque M, Morissette M, Di Paolo T (2010): Steroids-dopamine interactions in the pathophysiology and treatment of CNS disorders. CNS Neurosci Ther 16(3): e43-71

Sanfilipo M, Lafargue T, Rusinek H, Arena L, Loneragan C, Lautin A, Rotrosen J, Wolkin A (2002): Cognitive performance in schizophrenia: relationship to regional brain volumes and psychiatric symptoms. Psychiatry Res 116(1-2): 1-23

Saykin AJ, Gur RC, Gur RE, Mozley PD, Mozley LH, Resnick SM, Kester DB, Stafiniak P (1991): Neuropsychological function in schizophrenia. Selective impairment in memory and learning. Arch Gen Psychiatry 48(7): 618-24

Scharfetter C: Allgemeine Psychopathologie, 5. Auflage, Georg Thieme Verlag, Stuttgart 1996.

Schneider B: Antipsychotische Äquivalenzdosis für Paliperidon. Neuss-Norf, Janssen-Cilag GmbH, schriftliche Mitteilung 2010

Schultz SK, Miller DD, Oliver SE, Arndt S, Flaum M, Andreasen NC (1997): The life course of schizophrenia: age and symptom dimensions. Schizophr Res 23(1): 15-23 
Schulz P, Rey MJ, Dick P, Tissot R (1989): Guidelines for the dosage of neuroleptics. II: Changing from daily oral to long acting injectable neuroleptics. Int Clin Psychopharmacol 4(2): 105-14

Seeman MV (1996): The role of estrogen in schizophrenia. J Psychiatry Neurosci 21(2): 123-7

Seeman MV (1997): Psychopathology in women and men: focus on female hormones. Am J Psychiatry 154(12): 1641-7

Seeman MV (2004): Gender differences in the prescribing of antipsychotic drugs. Am J Psychiatry 161(8): 1324-33

Seeman MV (2009): Secondary effects of antipsychotics: women at greater risk than men. Schizophr Bull 35(5): 937-48

Seeman P, Weinshenker D, Quirion R, Srivastava LK, Bhardwaj SK, Grandy DK, Premont RT, Sotnikova TD, Boksa P, El-Ghundi M, et al. (2005): Dopamine supersensitivity correlates with D2High states, implying many paths to psychosis. Proc Natl Acad Sci U S A 102(9): 3513-8

Segarra R, Zabala A, Eguiluz JI, Ojeda N, Elizagarate E, Sanchez P, Ballesteros J, Gutierrez M (2010): Cognitive performance and smoking in first-episode psychosis: the selfmedication hypothesis. Eur Arch Psychiatry Clin Neurosci

Shimoda K, Someya T, Morita S, Hirokane G, Noguchi T, Yokono A, Shibasaki M, Takahashi $S$ (1999): Lower plasma levels of haloperidol in smoking than in nonsmoking schizophrenic patients. Ther Drug Monit 21(3): 293-6

Silvestri S, Negrete JC, Seeman MV, Shammi CM, Seeman P (2004): Does nicotine affect D2 receptor upregulation? A case-control study. Acta Psychiatr Scand 109(4): 313-7; discussion 317-8

Simpson GM, Angus JW (1970): A rating scale for extrapyramidal side effects. Acta Psychiatr Scand Suppl 212: 11-19

Simpson GM, Lee JH, Zoubok B, Gardos G (1979): A rating scale for tardive dyskinesia. Psychopharmacology 64(2): 171-179

Skarsfeldt T (1996): Differential effect of antipsychotics on place navigation of rats in the Morris water maze. A comparative study between novel and reference antipsychotics. Psychopharmacology (Berl) 124(1-2): 126-33

Skre I, Onstad S, Torgersen S, Kringlen E (1991): High interrater reliability for the Structured Clinical Interview for DSM-III-R Axis I (SCID-I). Acta Psychiatr Scand 84(2): 167-73

Spohn HE, Strauss ME (1989): Relation of neuroleptic and anticholinergic medication to cognitive functions in schizophrenia. J Abnorm Psychol 98(4): 367-80

Stahl SM, Grady MM (2004): A critical review of atypical antipsychotic utilization: comparing monotherapy with polypharmacy and augmentation. Curr Med Chem 11(3): 313-27

Steinkamp RC, Cohen NL, Gaffey WR, McKey T, Bron G, Siri WE, Sargent TW, Isaacs E (1965): Measures of body fat and related factors in normal adults. II. A simple clinical method to estimate body fat and lean body mass. J Chronic Dis 18(12): 1291-307

Stuss DT, Levine B (2002): Adult clinical neuropsychology: lessons from studies of the frontal lobes. Annu Rev Psychol 53: 401-33

Sumiyoshi T, Hasegawa M, Jayathilake K, Meltzer HY (1997): Sex differences in plasma homovanillic acid levels in schizophrenia and normal controls: relation to neuroleptic resistance. Biol Psychiatry 41(5): 560-6

Swartz MS, Stroup TS, McEvoy JP, Davis SM, Rosenheck RA, Keefe RS, Hsiao JK, Lieberman JA (2008): What CATIE found: results from the schizophrenia trial. Psychiatr Serv 59(5): 500-6

Szymanski S, Lieberman JA, Alvir JM, Mayerhoff D, Loebel A, Geisler S, Chakos M, Koreen A, Jody D, Kane J, et al. (1995): Gender differences in onset of illness, treatment response, course, and biologic indexes in first-episode schizophrenic patients. Am J Psychiatry 152(5): 698-703

Tamminga CA, Stan AD, Wagner AD (2010): The hippocampal formation in schizophrenia. Am J Psychiatry 167(10): 1178-93 
Tang YL, Gillespie CF, Epstein MP, Mao PX, Jiang F, Chen Q, Cai ZJ, Mitchell PB (2007): Gender differences in 542 Chinese inpatients with schizophrenia. Schizophr Res 97(13): $88-96$

Tang YL, Mao P, Li FM, Li W, Chen Q, Jiang F, Cai ZJ, Mitchell PB (2007): Gender, age, smoking behaviour and plasma clozapine concentrations in 193 Chinese inpatients with schizophrenia. Br J Clin Pharmacol 64(1): 49-56

Tauscher J, Kapur S (2001): Choosing the right dose of antipsychotics in schizophrenia: lessons from neuroimaging studies. CNS Drugs 15(9): 671-8

Taylor DC (1969): Differential rates of cerebral maturation between sexes and between hemispheres. Evidence from epilepsy. Lancet 1969,2: 140-2

Tewes U: German Adaption of the Revised Wechsler Adult Intelligence Scale (HAWIE-R), Hans Huber, Bern 1991.

Toomey R, Faraone SV, Seidman LJ, Kremen WS, Pepple JR, Tsuang MT (1998): Association of neuropsychological vulnerability markers in relatives of schizophrenic patients. Schizophr Res 31(2-3): 89-98

Uchida H, Mamo DC (2009): Dosing of antipsychotics in schizophrenia across the lifespectrum. Prog Neuropsychopharmacol Biol Psychiatry 33(6): 917-20

Uchida H, Suzuki T, Mamo DC, Mulsant BH, Tanabe A, Inagaki A, Watanabe K, Yagi G, Tomita M (2008): Effects of age and age of onset on prescribed antipsychotic dose in schizophrenia spectrum disorders: a survey of 1,418 patients in Japan. Am J Geriatr Psychiatry 16(7): 584-93

Usall J, Suarez D, Haro JM (2007): Gender differences in response to antipsychotic treatment in outpatients with schizophrenia. Psychiatry Res 153(3): 225-31

van Zelst C (2009): Stigmatization as an environmental risk in schizophrenia: a user perspective. Schizophr Bull 35(2): 293-6

Ventura J, Hellemann GS, Thames AD, Koellner V, Nuechterlein KH (2009): Symptoms as mediators of the relationship between neurocognition and functional outcome in schizophrenia: a meta-analysis. Schizophr Res 113(2-3): 189-99

Vermeulen A, Kaufman JM, Goemaere S, van Pottelberg I (2002): Estradiol in elderly men. Aging Male 5(2): 98-102

Vinogradov S, Fisher M, Warm H, Holland C, Kirshner MA, Pollock BG (2009): The cognitive cost of anticholinergic burden: decreased response to cognitive training in schizophrenia. Am J Psychiatry 166(9): 1055-62

Waters FA, Badcock JC (2010): First-rank symptoms in schizophrenia: reexamining mechanisms of self-recognition. Schizophr Bull 36(3): 510-7

Wechsler D: Wechsler Memory Scale - 3rd edition (WMS-III), The Psychological Corporation, Harcourt 1998.

Weickert TW, Goldberg TE (2005): First- and second-generation antipsychotic medication and cognitive processing in schizophrenia. Curr Psychiatry Rep 7(4): 304-10

Weinberger DR, Egan MF, Bertolino A, Callicott JH, Mattay VS, Lipska BK, Berman KF, Goldberg TE (2001): Prefrontal neurons and the genetics of schizophrenia. Biol Psychiatry 50(11): 825-44

Wilk CM, Gold JM, Humber K, Dickerson F, Fenton WS, Buchanan RW (2004): Brief cognitive assessment in schizophrenia: normative data for the Repeatable Battery for the Assessment of Neuropsychological Status. Schizophr Res 70(2-3): 175-86

Winterer G (2010): Why do patients with schizophrenia smoke? Curr Opin Psychiatry 23(2): 112-9

Wittchen H-U, Zaudig M, Fydrich T: SCID-I: Structured Clinical Interview for DSM-IV Disorders, Hogrefe, Göttingen 1997.

Woods SW (2003): Chlorpromazine equivalent doses for the newer atypical antipsychotics. J Clin Psychiatry 64(6): 663-7 
Woodward ND, Purdon SE, Meltzer HY, Zald DH (2005): A meta-analysis of neuropsychological change to clozapine, olanzapine, quetiapine, and risperidone in schizophrenia. Int J Neuropsychopharmacol 8(3): 457-72

www.matrics.ucla.edu. (2010). Internetseite von MATRICS (Measurement And Tratment Research to Improve Cognition in Schizophrenia).

Wyatt RJ (1991): Neuroleptics and the natural course of schizophrenia. Schizophr Bull 17(2): 325-51

Xiang YT, Weng YZ, Leung CM, Tang WK, Ungvari GS (2007): Clinical and social determinants of antipsychotic polypharmacy for Chinese patients with schizophrenia. Pharmacopsychiatry 40(2): 47-52

Zanarini MC, Frankenburg FR (2001): Attainment and maintenance of reliability of axis I and II disorders over the course of a longitudinal study. Compr Psychiatry 42(5): 369-74

Zec RF, Trivedi MA (2002): The effects of estrogen replacement therapy on neuropsychological functioning in postmenopausal women with and without dementia: a critical and theoretical review. Neuropsychol Rev 12(2): 65-109

Zevin S, Benowitz NL (1999): Drug interactions with tobacco smoking. An update. Clin Pharmacokinet 36(6): 425-38

Zimmermann P, Fimm B: Test for Attentional Performance (Translated from German (TAP)), PSYTEST, Freiburg 1993. 


\section{DANKSAGUNG}

Bei all denen, die zum Gelingen dieser Arbeit beigetragen haben, möchte ich mich herzlich bedanken.

Mein besonderer Dank gilt Frau Prof. Dr. Dr. Hannelore Ehrenreich für die Ermöglichung der Dissertation sowie die fachliche Betreuung und konstruktive Kritik. Unter dem Ansatz "from bench to bedside and back again" durfte ich als Teil ihrer Arbeitgruppe einen intensiven und umfassenden Einblick in hervorragende, aktuelle Forschung gewinnen.

Dem gesamten GRAS-Team mit Claudia Bartels, Martin Begemann, Heidi Friedrichs, Martin Fungisai Gerchen, Mohammad Ghorbani, Stefan Gutwinski, Constanze Hilmes, Henning Krampe, Richard Leppert, Katja Ribbe, Julia Sowislo, Sabina Stawicki, Maren Stödtke danke ich für die geduldige und akribische Datenerhebung und -verwaltung. Auf Ideen, fundiertem Fachwissen und dem Engagement eines jeden einzelnen basiert die GRAS-Datenbank, welche die Grundlage dieser Arbeit darstellt. Ganz besonders möchte ich Richard Leppert für den gemeinsamen Weg innerhalb und außerhalb des Max-Planck-Institutes danken.

Allen kooperierenden Zentren danke ich für die Hilfe der Probandenrekrutierung und der Unterstützung der GRAS-Studie.

In der Hoffnung auf eine greifende Therapie der Schizophrenie möchte ich diese Arbeit den teilnehmenden Probanden sowie allen an Schizophrenie leidenden Menschen widmen. 


\section{LEBENSLAUF}

Am 24.11.1981 bin ich als zweites Kind der Eheleute Waltraud Knop-Szuszies und Klaus Szuszies in Bielefeld geboren.

Von 1988 bis 1992 besuchte ich die Grundschule in Herford - Elverdissen. 1992 wechselte ich zum Ravensberger Gymnasium der Stadt Herford, das ich im Jahr 2001 mit dem Abitur verließ. Meinen Zivildienst leistete ich im Lukas-Krankenhaus in Bünde ab. Im Oktober 2002 begann ich das Studium der Humanmedizin an der Georg-August-Universität in Göttingen. Von Juli 2005 bis zum September 2006 war ich im Rahmen der Dissertation in der Division klinische Neurowissenschaften im Max-Planck-Institut für Experimentelle Medizin in Göttingen bei Prof. Dr. Dr. Ehrenreich beschäftigt. Nach der Wiederaufnahme des Studiums in Göttingen sammelte ich im praktischen Jahr klinische Erfahrung in Bremen, der Schweiz (Nidwalden) und den Niederlanden (Rotterdam). Im Dezember 2009 bestand ich die ärztliche Prüfung und bin seit Januar 2010 approbierter Arzt. Im Juni 2010 wurde mein Sohn geboren. Seit April 2011 befinde ich mich in internistischer Facharztweiterbildung in der Abteilung Hämatologie und Onkologie der Universitätsmedizin Göttingen. 